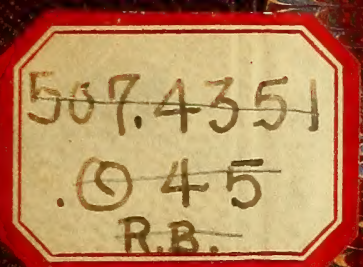

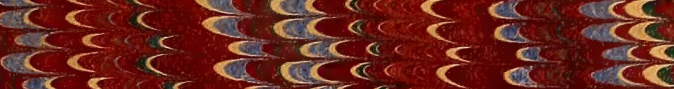

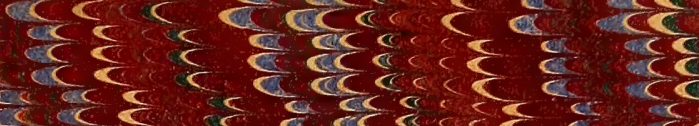

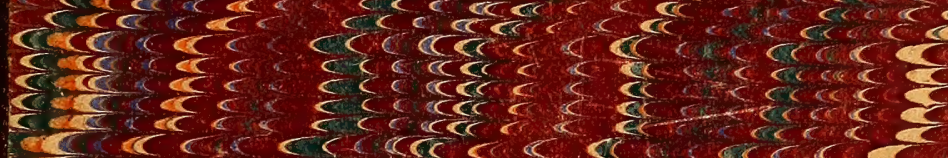

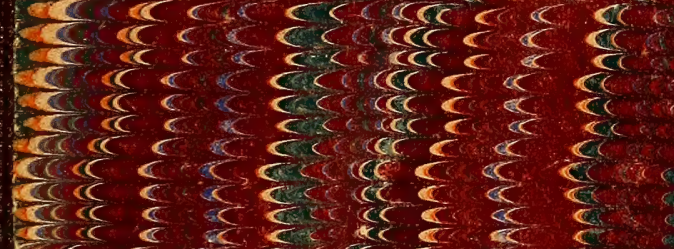

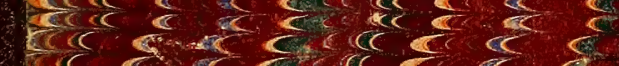

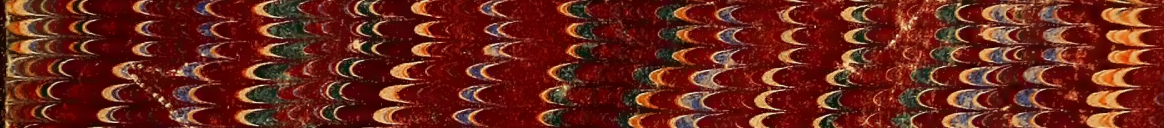

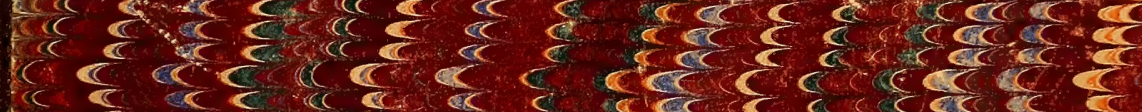

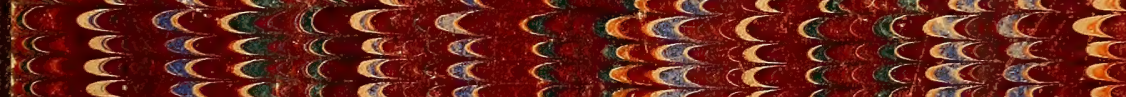

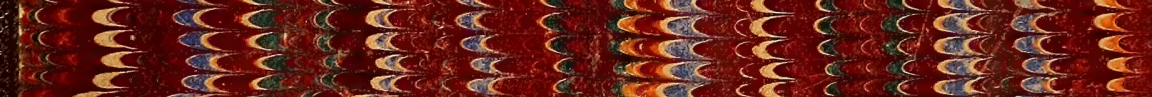

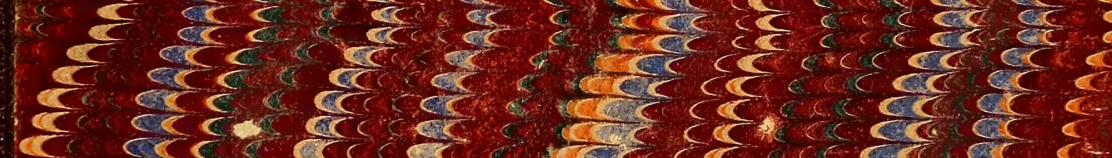
c

हैं

है

हो त्र टा दो बरे बर्दर बर बर बर tces $k \mathbb{x} \cdot$

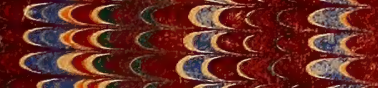
ars $x<$ $\cos 2 \frac{1}{2}$ $\cos a x$ ces $\frac{x}{6}$

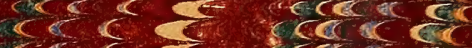
रक है। car ex $x$ s es $x<c k$ scen

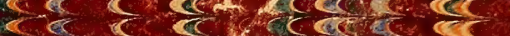
- ats $5 \mathbb{5}$ cis बिर $\mathrm{Cl}$ cas ce

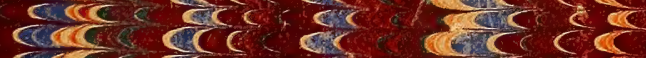

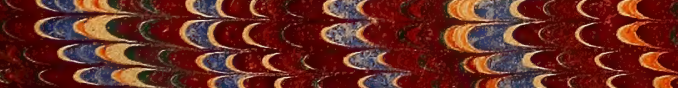

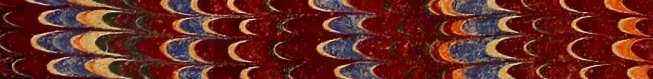

कर बर बरे \& c 


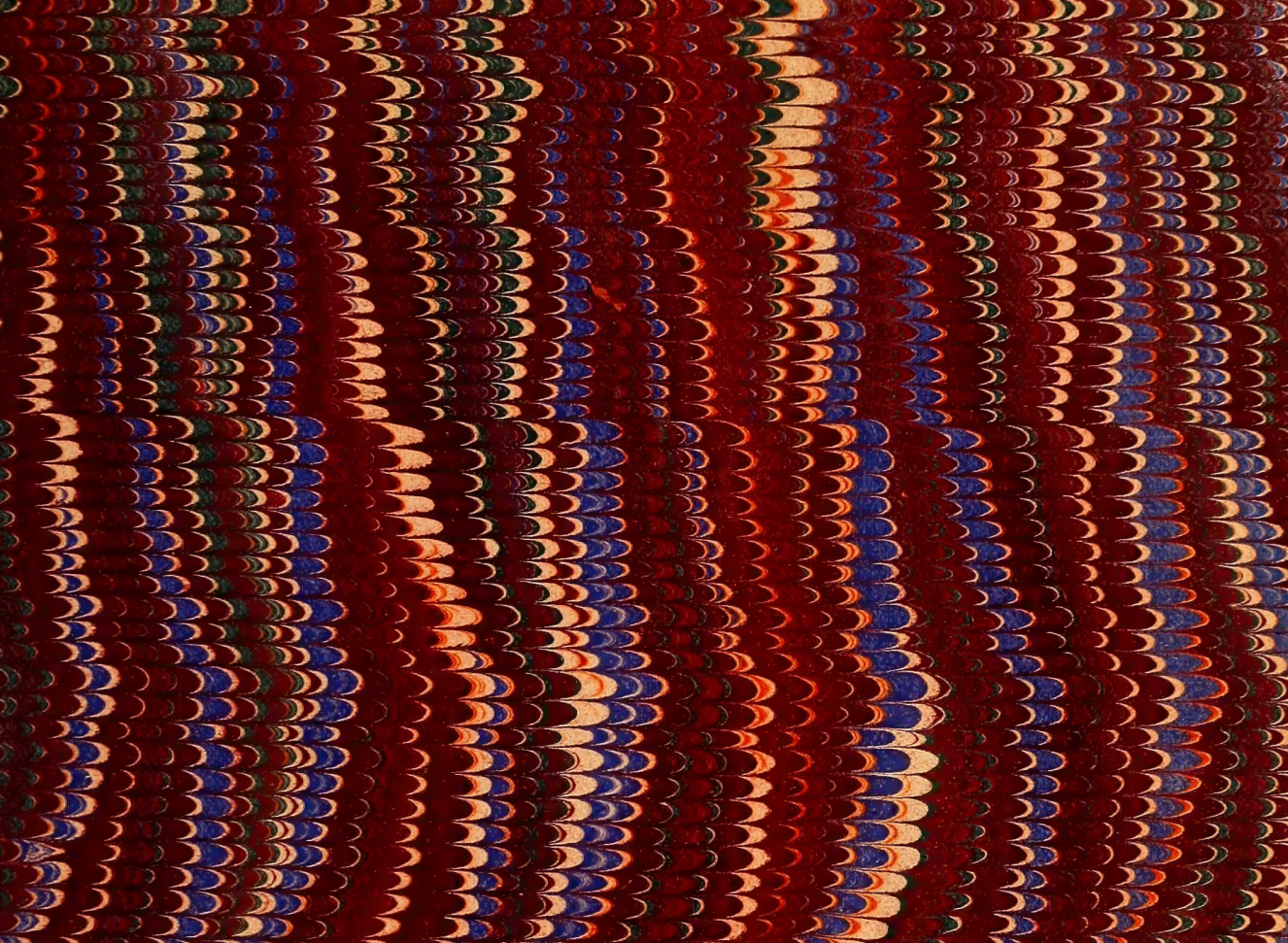









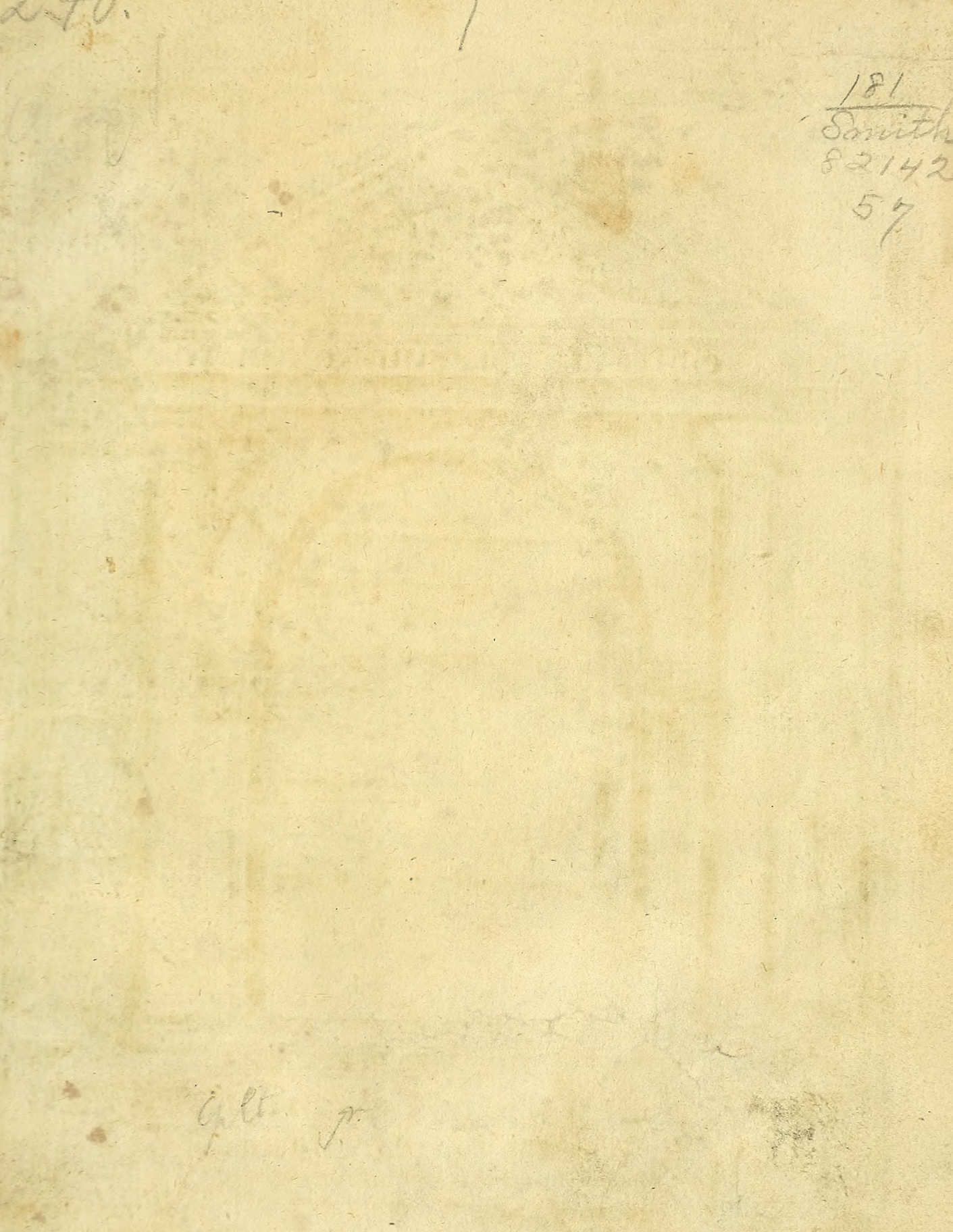




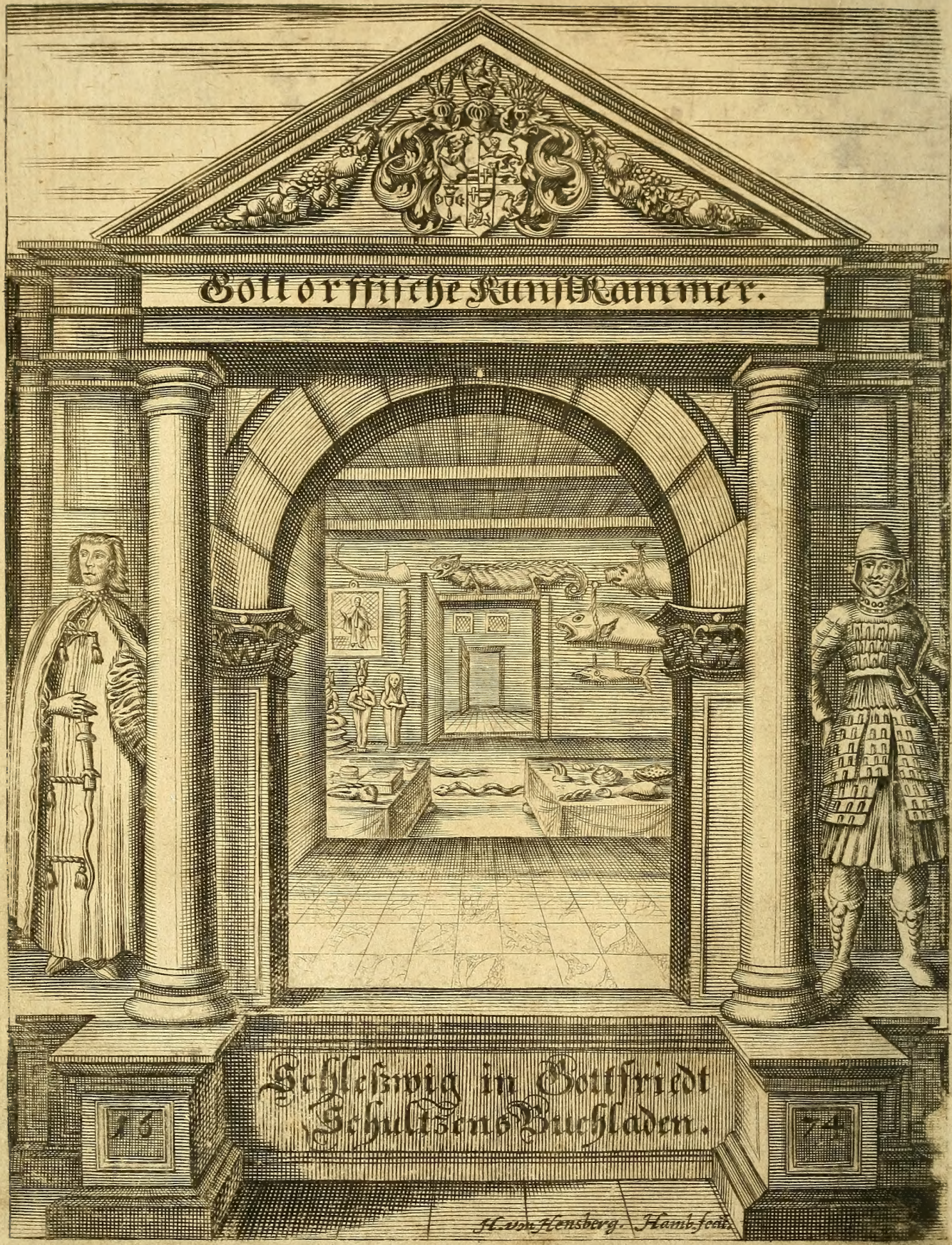




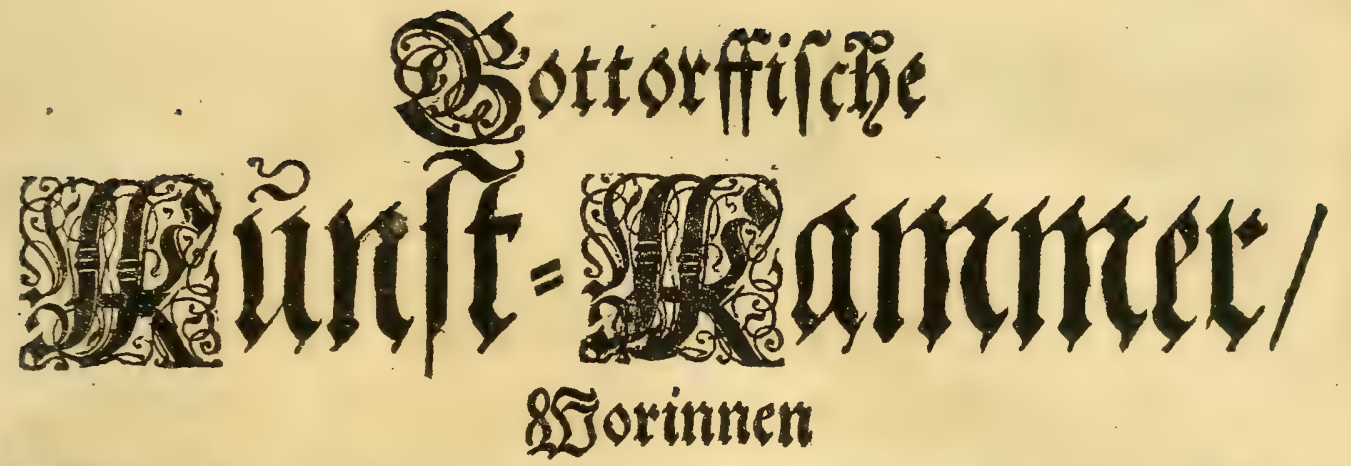

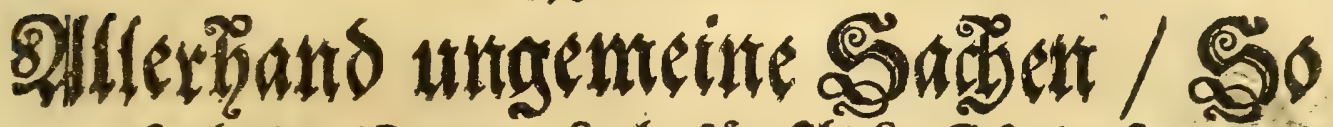

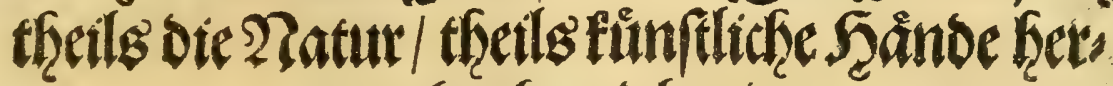
borgebradft uno bereitet.

Vor Diefem

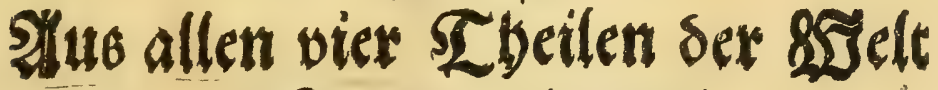
zutarmmen getwagen/

uino

Bor einigen Safiren beff̧rieben/

2uch mitbehörigen 2 upfern gezieret

Duturef

Adam Olearium, $8 \mathfrak{g e i l . B i b l i o t h e c a r i u m ~}$

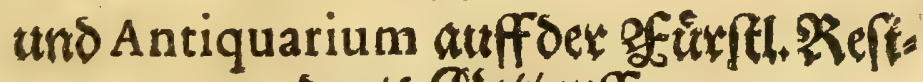

Dento Gottorff.

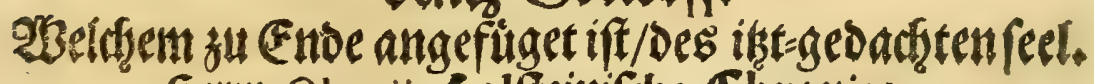

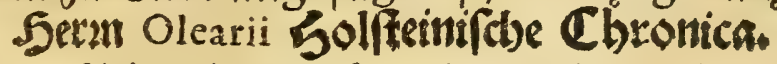

Iniefgo aber úberfehen/uno sum andern mal gedructet/

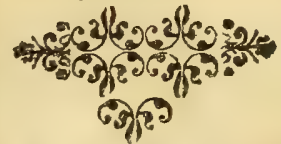

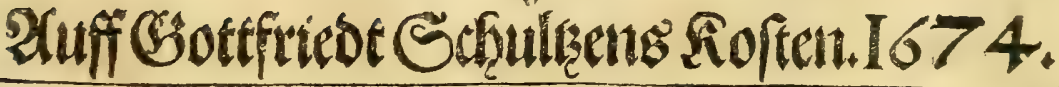

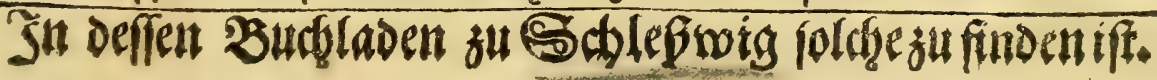


Beridest

In ben 2 Butchbinter

rsobiunde Supffer zu binden find.

$\begin{array}{lllllll}\text { Tab. } & \\ \text { II. }\end{array}$

149623 


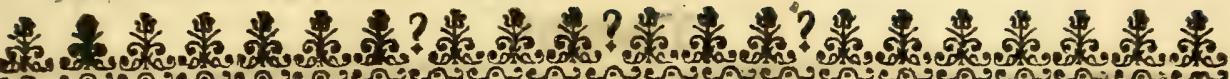

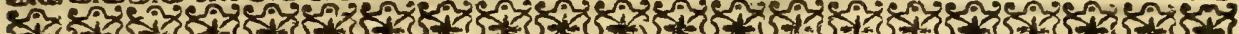

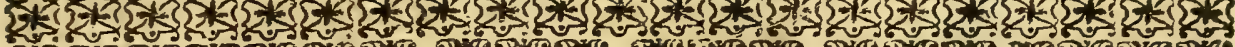

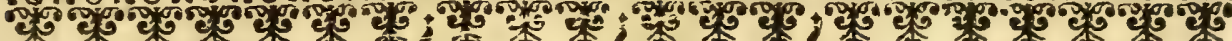

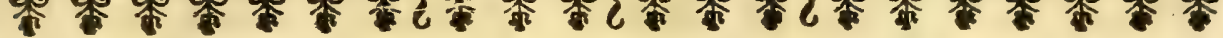

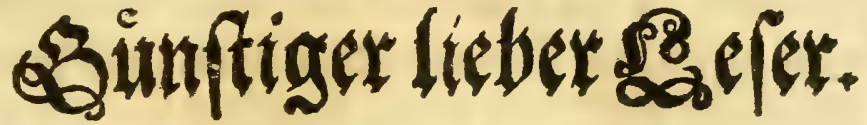

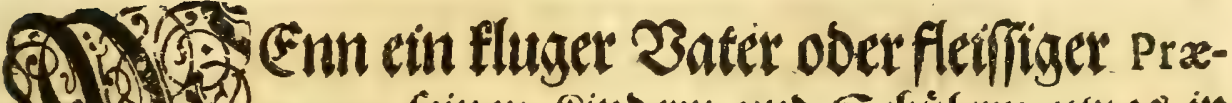
1. If ceptor feinen sindern und Enfütern erwas in

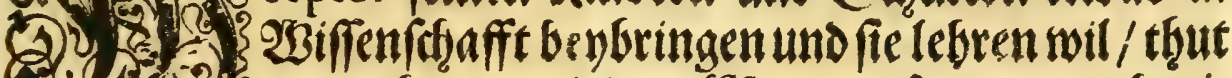
W.

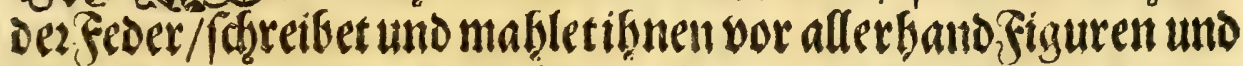

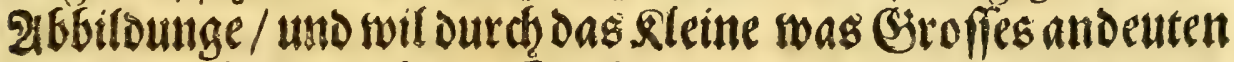

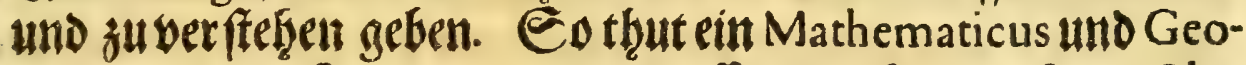
metra. Ein Aftronomus zeiget auff eintem fleinen Şand Globo Coelefti Die Berffiaffentheit Des groffen Shinmels nnit allen Feinen fitgtbaren SCorpern/oa ein Punct einen groffenen Etern bedeutet. Imgleidgen audd ein Geographus bilbet auffe einer

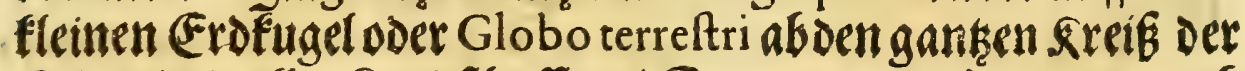

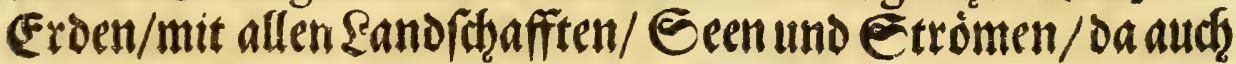
ein Punct enne E tadt/ente Einte einen Strom/und ein Plakg el= nes Daumens breit eine ganke breite See abbirsen mub.

EGen auff foldche 2art bandelt unfer allgemeiner 3ater im

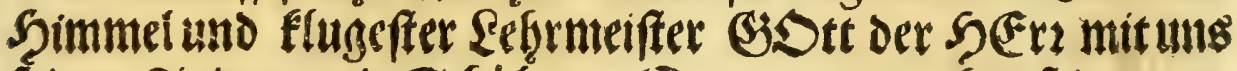
Feenten Sindern und Erfhülern. Tanner unts neben feentem ges

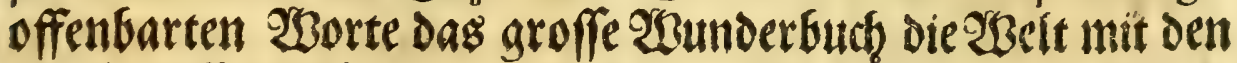

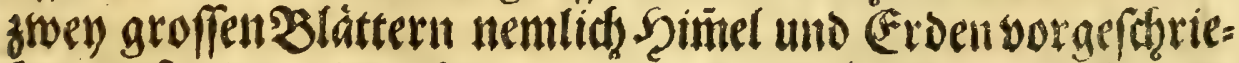

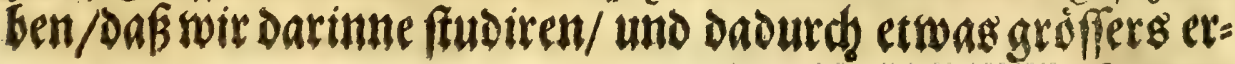




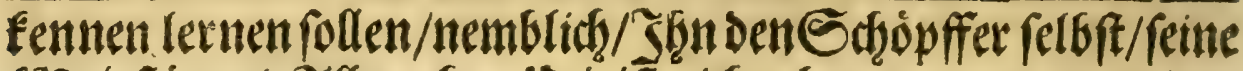

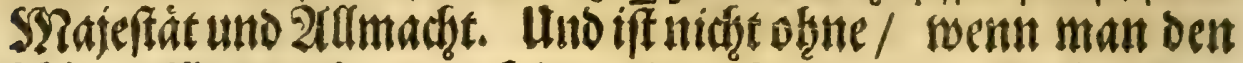

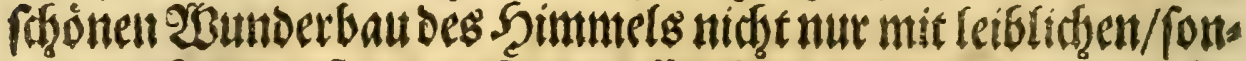

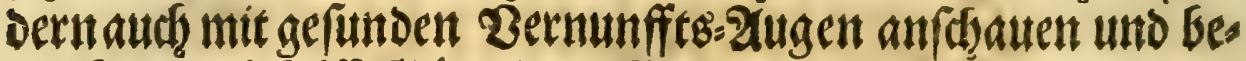

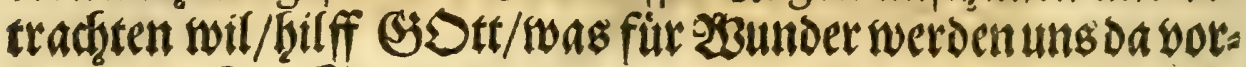
fommen. Die Sterne/Die wir von uns etlithetaufeno Syeilen

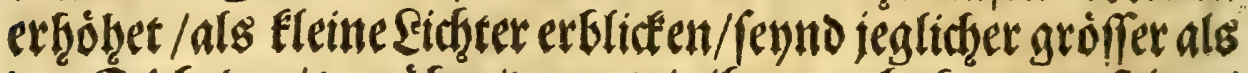
Der Eroboden/ woriber/wenn wir ifgnen nabe fommen folten/

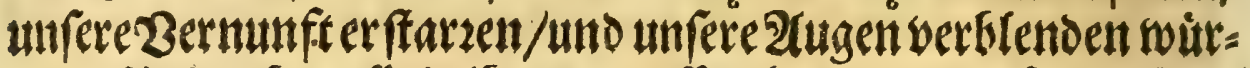

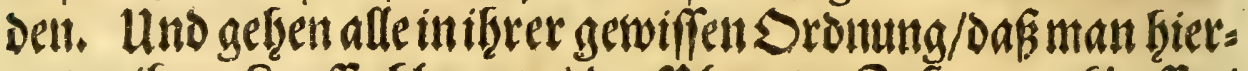
unten ifren \&auff ablernen / Der Planten Bufammenfinffel Entgegenfersunge und finfterniffe zuwor/ebe fie gerdetgen/be=

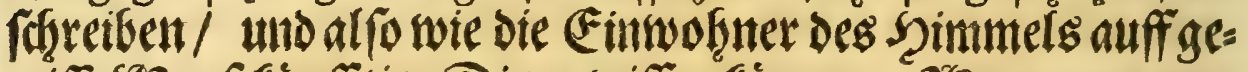

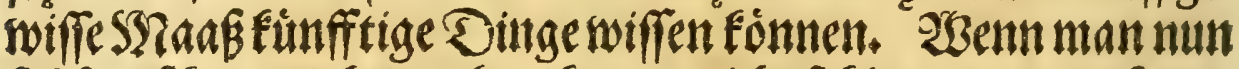

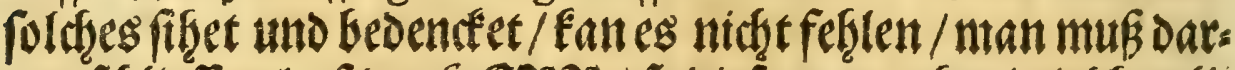

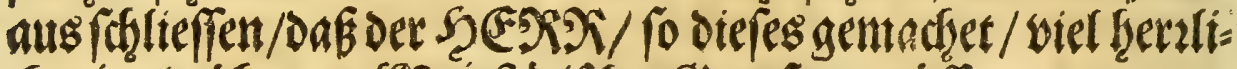

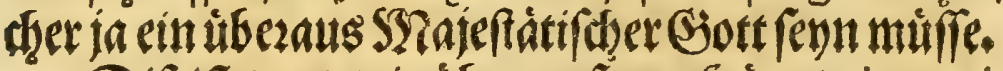

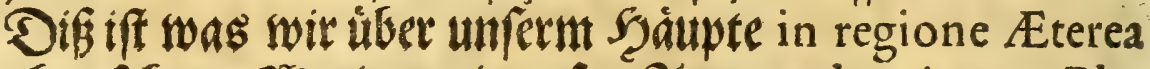

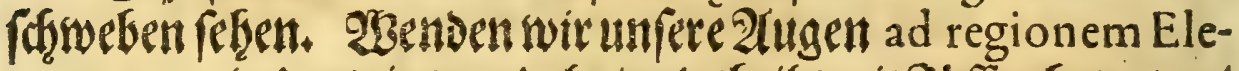
mentarem, in der wir wandeln/uno theils mit füffen Eetreten/

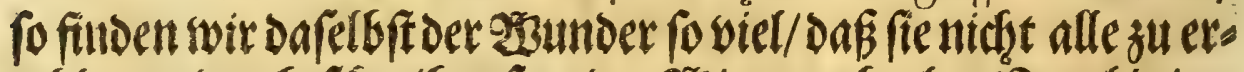

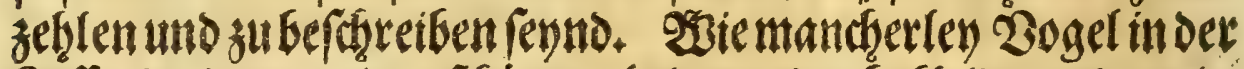
Eufft/ oa immer einer fogoner als der ander befleider und gezies

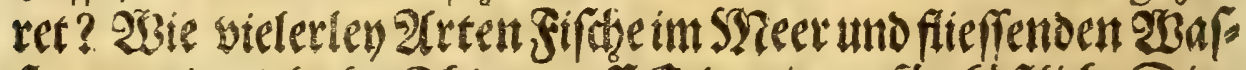

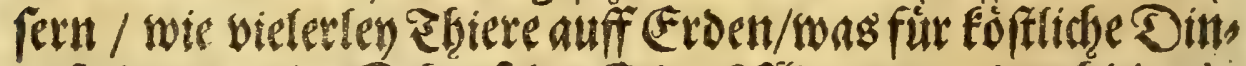

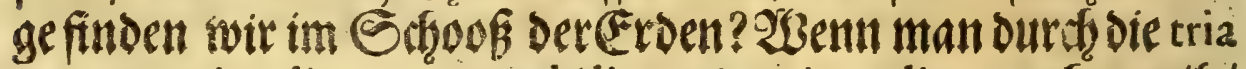
regna, animalium, vegetabilium \& mineralium getgen wil/ 


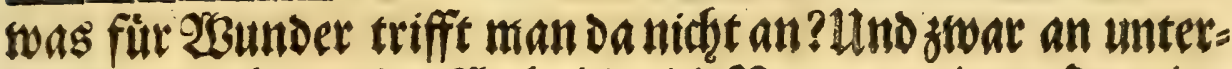

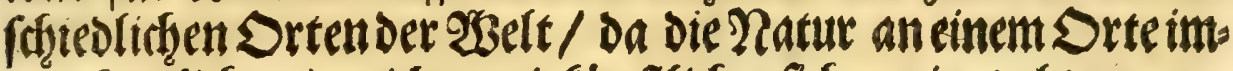
mer beralicher / reidber und fünftlicher fith erzeiget als am ant: Dem/Darwon die Siftorien/fgreiber/2aturfundiger und Chymici zu fagen wiffen. Daf man billid mit Davio ausurs: fen mag. O Domine Dominus nofter, quam admirabile eft

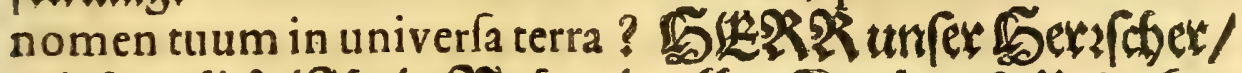

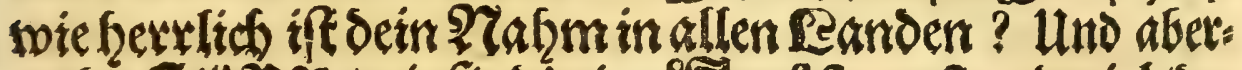

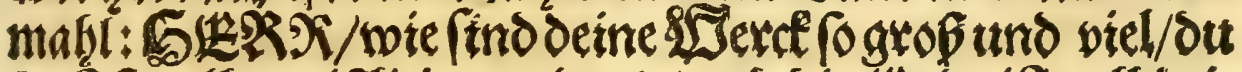

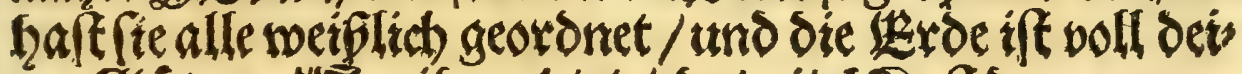

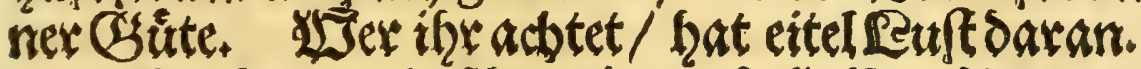

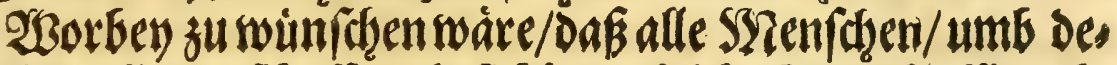
rer willen alles er/ḑaffen/luft batten foldge berrlide

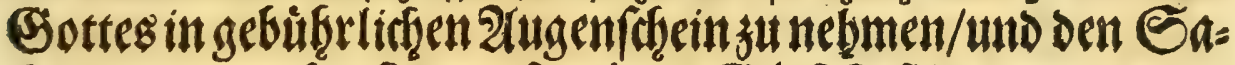

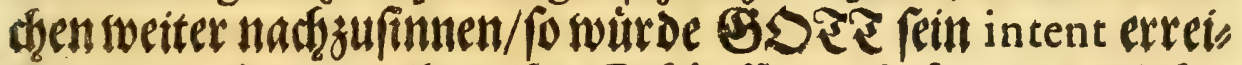
then/unt als der miloreiche Safopfier geliebet uno gelobet werden. Daryut bermatinet aud Paulus.

arber es berbălt fidf mit uns / wiemit den snaben in bet

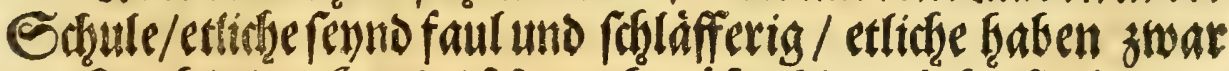
Das Buthinder Şano/ fehen aber úberbin/etliche ftedfendas Dutch unter die Sandx / gaffen auff andere Sachen/ und nef. men frembore Ciaucteler vor/etltbe aber/fo gute ingenia und Euft was zulernen faben/laffen esibuth/worauffife gewiefen werben/angelegen fenn. Eogefer es auth in ber grofien 2 elt:

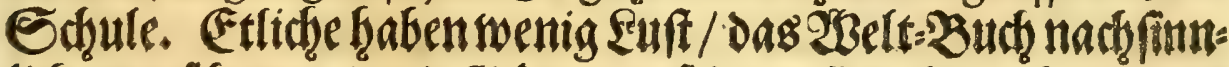

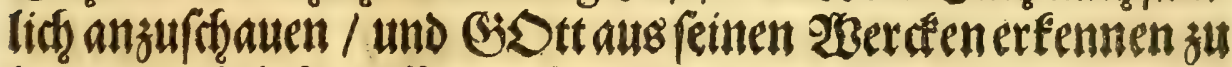
lernen; Etlidgegaffennadg ambern verbotenen Dingen/und 


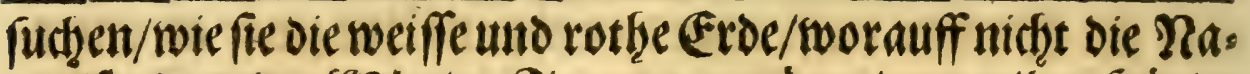

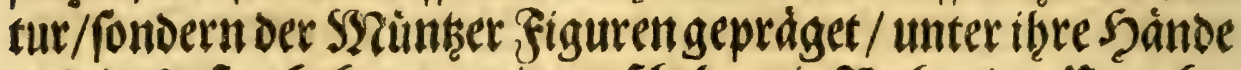

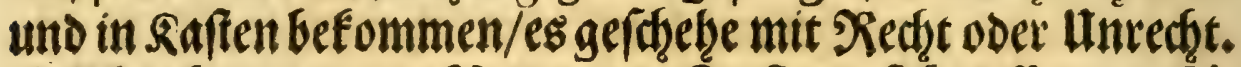
Etliche aber / berer ?zatur uno Berftand fith auff etwas ho. bers erftrectet / Gabentbre meiffe Beliebung und Ergeksung/

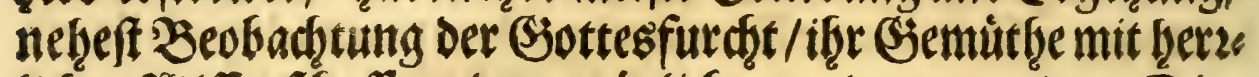
lidgen 2Biffen/d affiten ber natirlichen und ungemeinen Din: gen zuzieren / uno fo viel in diefer Oterblidǵfeit zugelafien

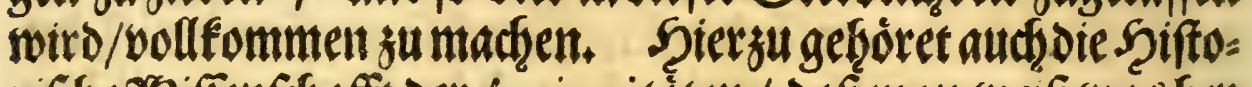

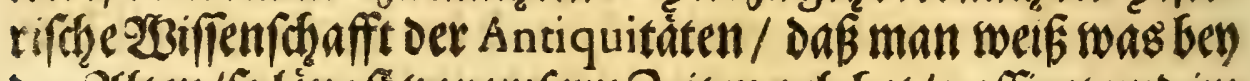
Den alten/Fo längft vor unfern Zeten gelebet/ paffiret und int Ge brauch gewe

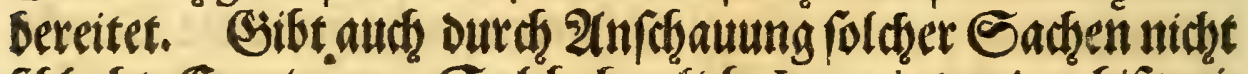

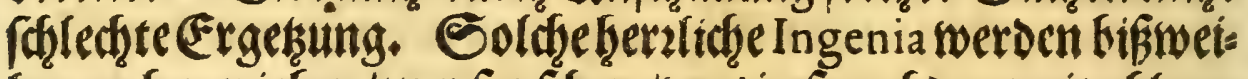
len auds getrieben/zu erfor/dhen/was in fremboen weit ableges nen Eanden anzutreffen. Daber begibt fidd mandjer mit grof

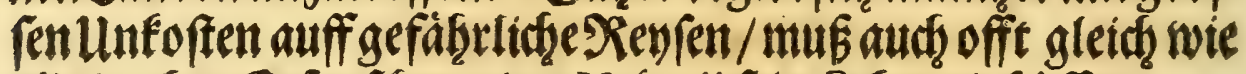
Plinius bey Erforfogung des Veluvii Fein Eeben einburfen.

Soldgen Eieb bebern aber fan guteg theils geţolffen wer: Den/wenn fie ait gewiffe Derter fommen / Da man fordhe heralis dhe/rare/wounderbare und fremboe Sadgen inden Labinetten/ Mufreis und \&unft: :ammern zufammen getragelt / findet / Da

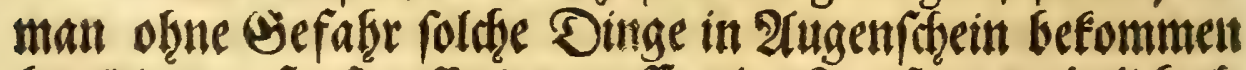

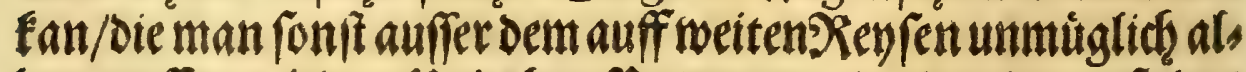
le antreffent wird. Uno thun Potentaten/ und andere / fo des Bermugens fenno / wol und loblidg daran / Daş fie zu Erfor.

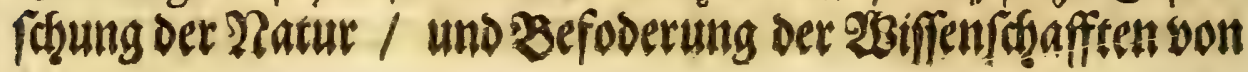


Dorrebe.

Denfelbigen/feine Untoften fparen. Ein foldher frengebiger Serz iftin diefem fall geweeren Alexander Magnus, weldfer Dem

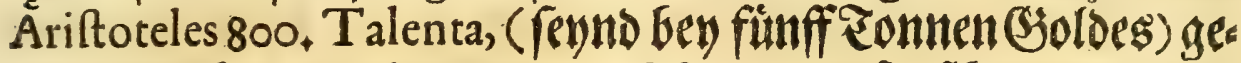
geben/umb nut die ?aturen der sgiere zuerforfden uno zu be

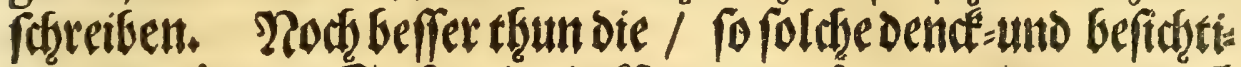

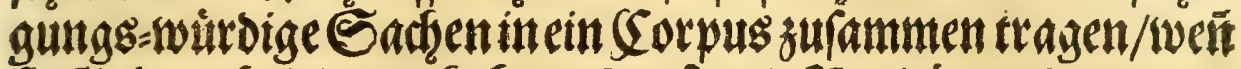
fie Şelegentbeit Darzu baben/ \$unft:uno Raritaten=-Sammeru

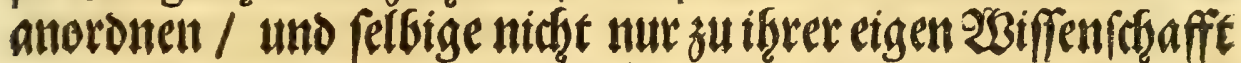

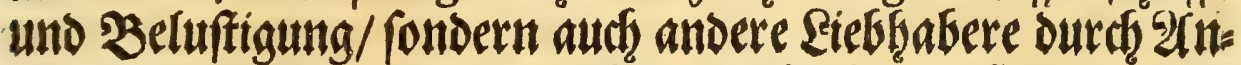
fóauten Derfelben genteffen laffen/2kie foldgeg an feintem orte

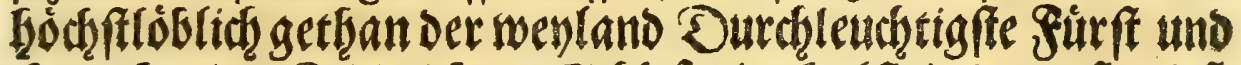

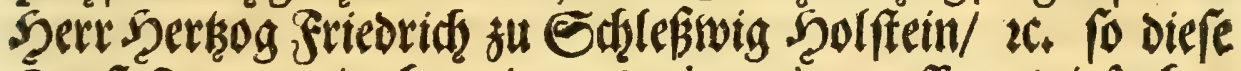

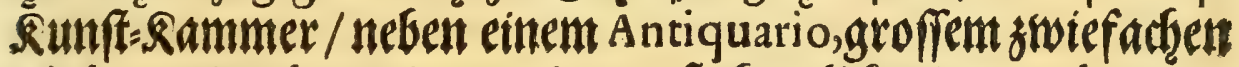
Globo uno Sphra Copernicana, fo bertlidge Opera thauma turgica bey oer Peftoents Sottorff angeridhter. Die Rumf. sammer betreffend/Gat biefelbige ibren Infang wonder weito

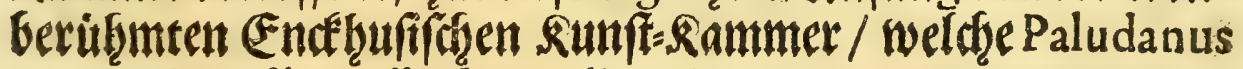
Der weylatio fürtrefflidfe Medicus in Egyptentumb andern A u-

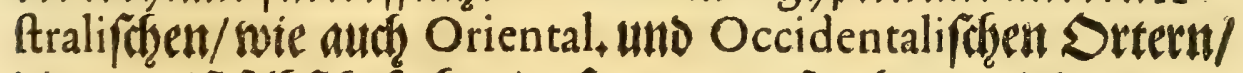
Diecr meiff felbft befuchet / zufammen gefamtet / und in ein ant: rebentlith Corpus gefradfet. Won Paludani Erben aber bat fie fiodffermelter Surft erfaufit/ unt A n no I651. Durd mids ats Şolland in Solfein bringen uno auff der Reftoents Sot:

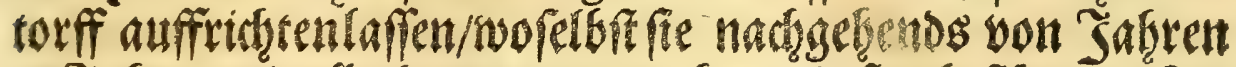
zu Sabren mit alleribano raren aud preciofen toftbaren Sa den wermeleret. Ind wird jo neben der foftbaren Bibliothecwondero Serun Sobn Sht: furft Durd b อ 


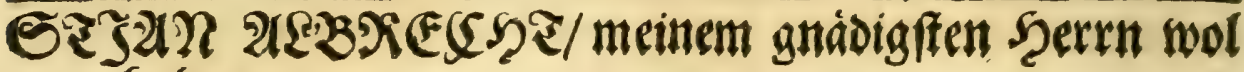
unterbalten.

Sonferend atth jetrigex zeit in den benachbarten

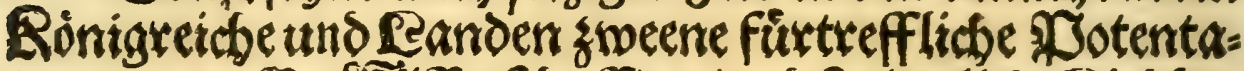

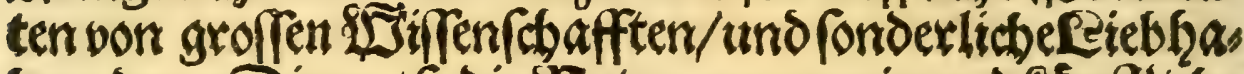

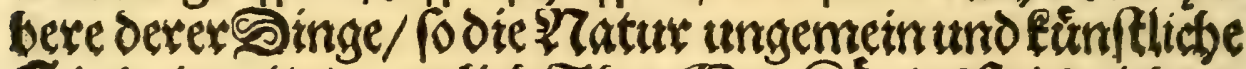

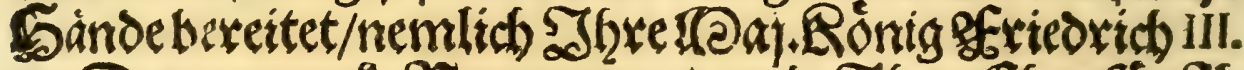

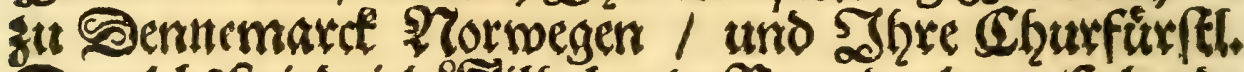

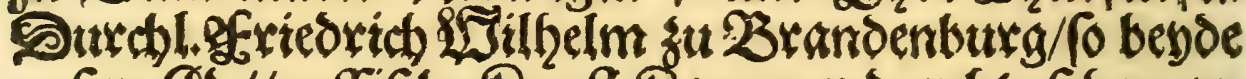

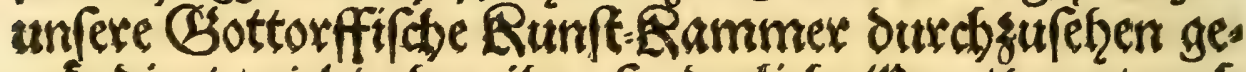

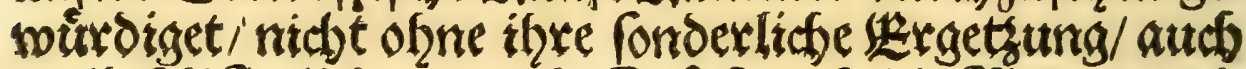

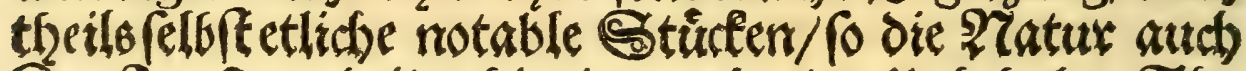

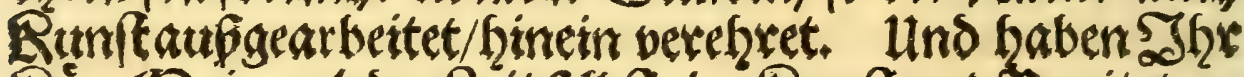

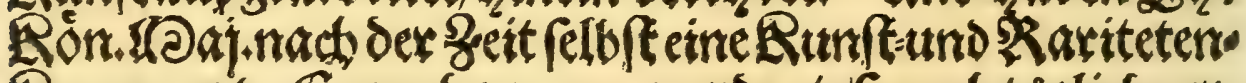
Rammer \&u Copenhagen angeoronet/fonoch tólich we

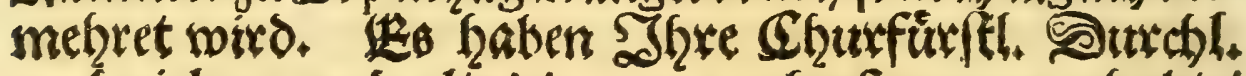
auth biel rare und alte Monumenta begfammen gefabt/

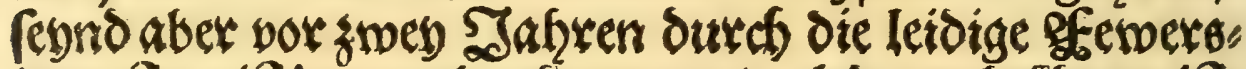
brumft meif darumb getommen/welches zubethlagenift.

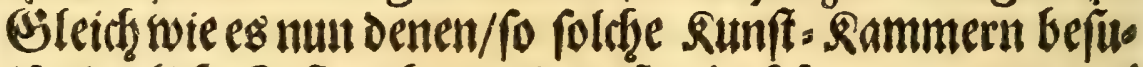
then/fonderlithe Euft giebet/in Dem fie gleithfam in einem nol

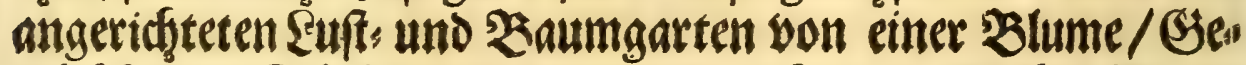

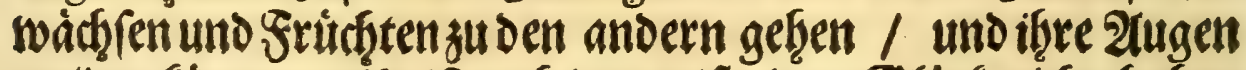

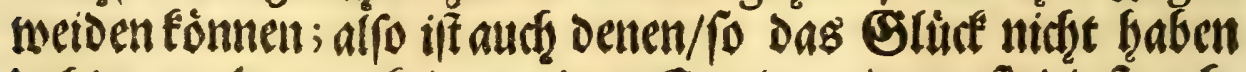
Dabin zu gelangen/feitte geringe Ergeksung/wenn fie oie fremb: Den Sachen mit Figuren abgezeidgnet reţen uno berchrieben les fen fơnnen. Sonverlithiftes eine angenelgme Sachef für bie/ 


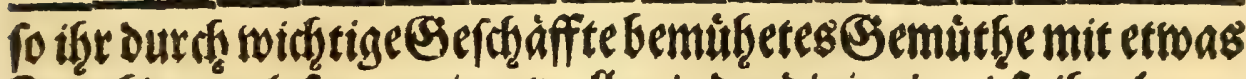

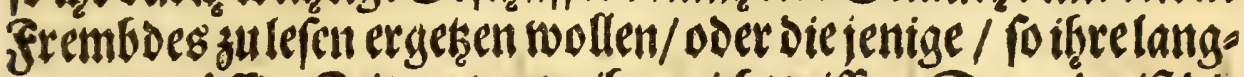
woerige mùffige 3 eit zu vertreiben nicht wiffen. Oann oa ift bie Biellheit uno 2ibroedfelunge ber frembdell und ungemeinen Eachen / Dás man inmer von einem auff Das ander fommen faut. Into meil es dann beiffet: Varietas delectat, int der Zerän -

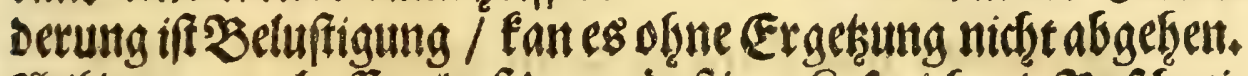

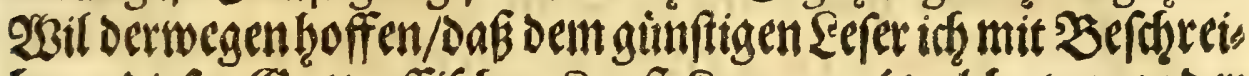

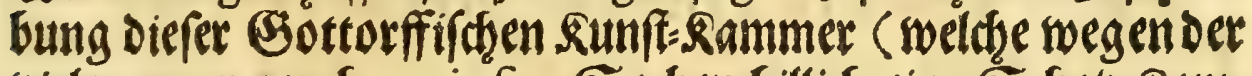

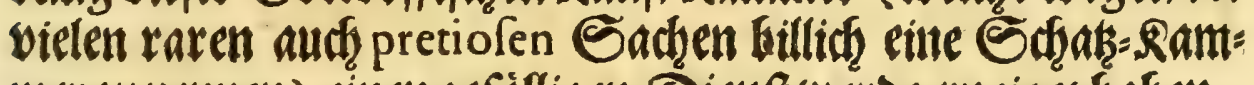
mer zunenuen) einengefälligen Dienft werde erzeiget haben.

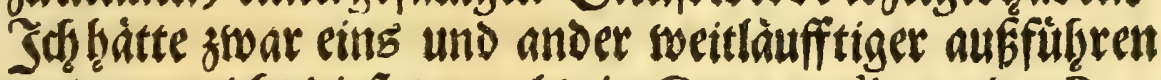
fóntren/wenunicht otefes nur als ein Compendium oder Pro-

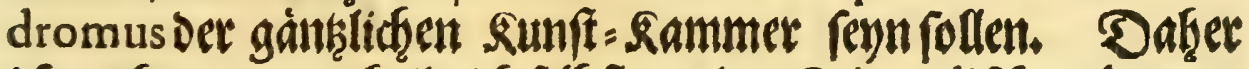

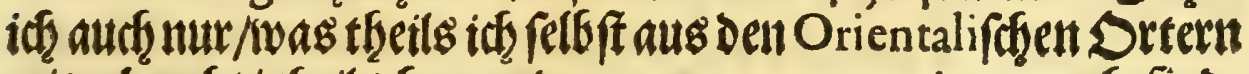
mitgebrad,t $/$ theils bey andern angezogenen Autoren befind:

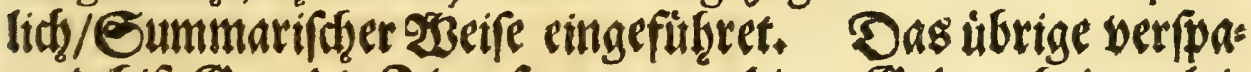
reno/bis Sntt die Feder ferner anzureten Gelegenteit verlei.

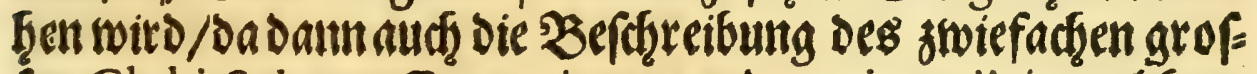
fen Globi,Sphrrx Copernicanx und A ntiquarii, in weldfem

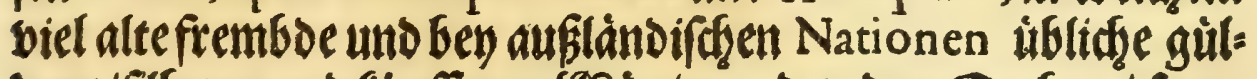
Dene/filberneund fupfferne Sriuntse und andere Oadhen / po an fat der Syiunttze gebraudgt werden / zu finden erfolgen follent.

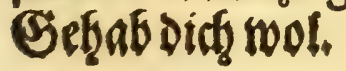

\section{Adam Olearius,}


In Pinacothecam Paludanæam à Batavis ad Serenisfimum Holfatix Principem Clarisfimi Olearii ductu translatam.

Drena qua Bataro venis optatisfima Ponto Grata ratis gratopondere fact a gravis.

Dedales vehis artis opus, mirabile quicquid

Orbis ab Eoo Belgicus orbe tulit;

Dic age: Dadaliden Jerens cum merferit undis;

Dedaleum cur boc mergere parcat opus?

Credite merfiffet, si non Olearide tutum

IJfet. Palladiis nil fcit obefJe Oleis.

\section{FRIED. CROCHEL;}

tunc temp. filiorum Serenisf. Princ. Informator. 
1.
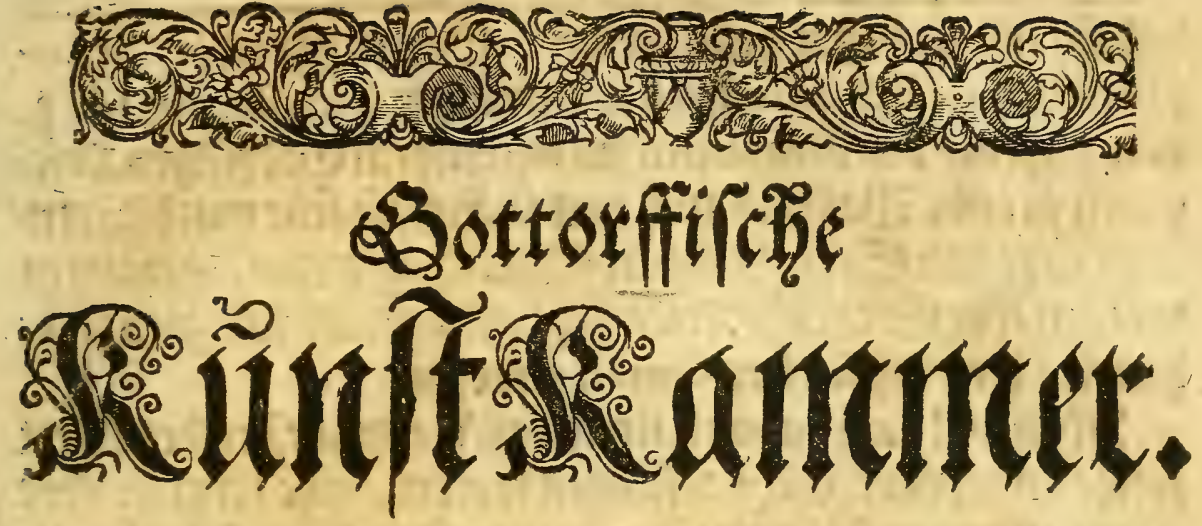

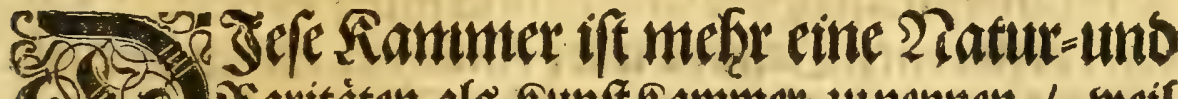

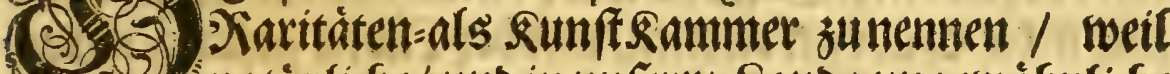

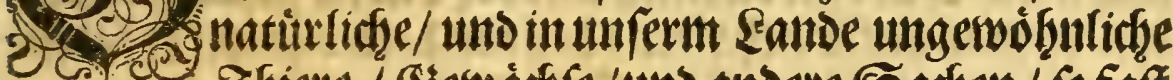

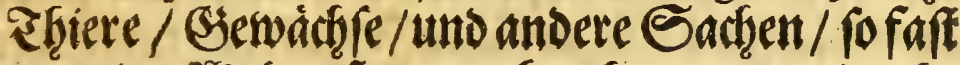
aus allen Orten der selt zufammen bradjet norden / mebr als fumftlidfe 2irbeit darein befindlich. Selbige feynd je=

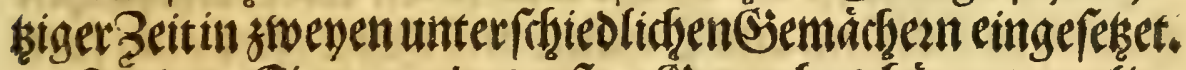

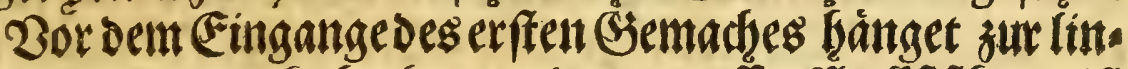

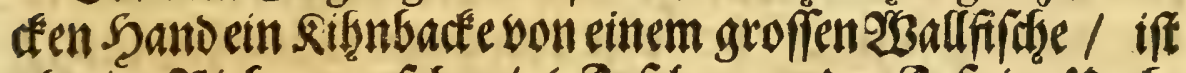
als eine Riebe anzu fang. Der fiffly aber mar 6z. Fuf lang / und die Syóbe 17. Fus. Eine lange Derfon funte in feinem auffigefperretent

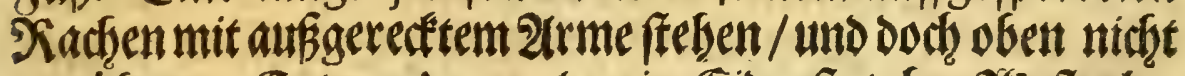

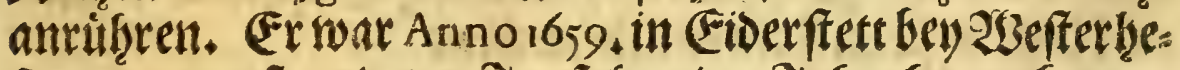

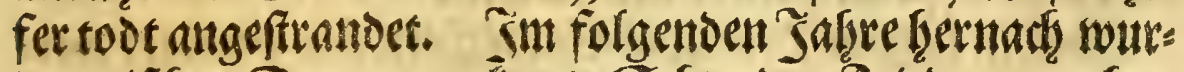
De zrwifhen Dennemara und Eafjweden Friebe gemachet. S6 das Zinftrandendiefes Firghes den frieden fo fl bedeutet a

baben/ 


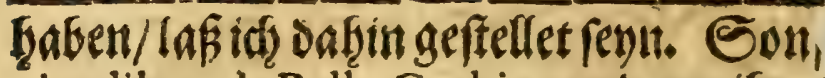
pius lib, 3 , de Bello Gothico unt aus if̧m $C$. $c_{+}$n. Dấ/als bey Bizantium imwärendeme

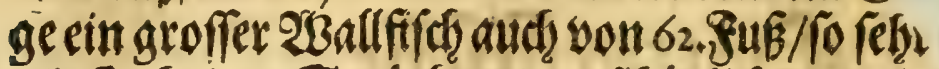

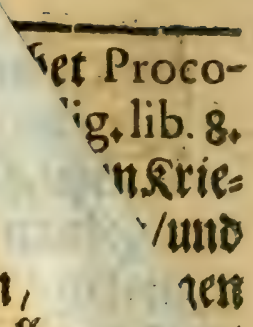

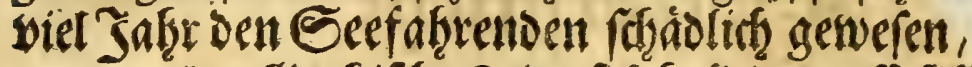
worden/Der Gothifdie srieg fidt Gald Darauff foll baben. Und dík fey das Omen gemejen.

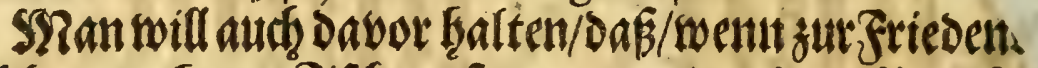
foldye ungebeure Fifde ge fangen werden/ eine unrubes Srieg andeuten foll/wie Crantzius infeiner Vandalia lib. \& c.17. vermentet, Dannes wäre Anno 1333. ein jungersball.

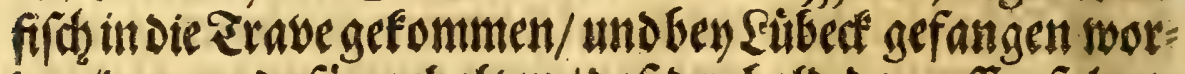
Den/Daman Dafür gelgalten / Da ber bald Daraufferfolgete

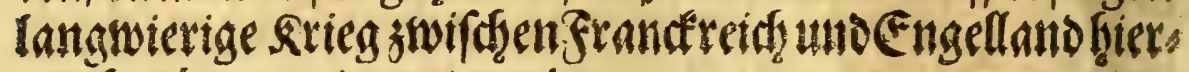

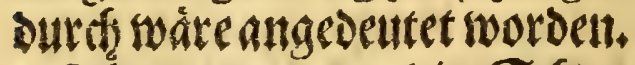

aris Anno i643. Die Erfineden einen untermuthlidfen

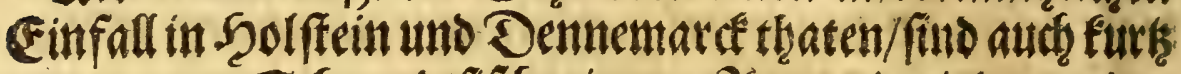

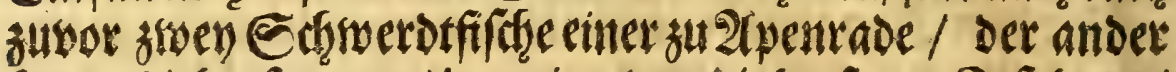
beym Riel gefangen / Der eine won biefen fo 1o. Sup lang/

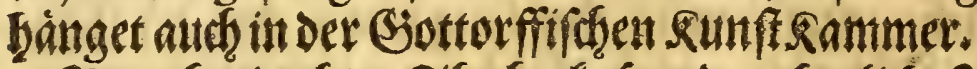

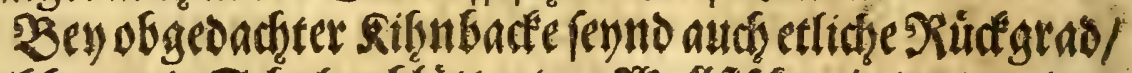

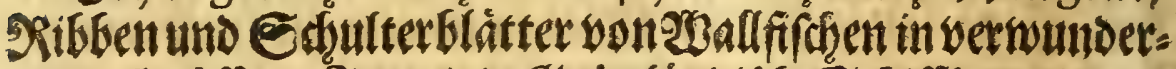

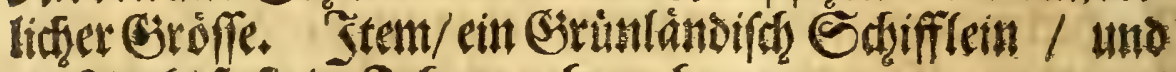
ein

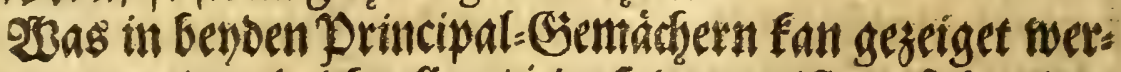

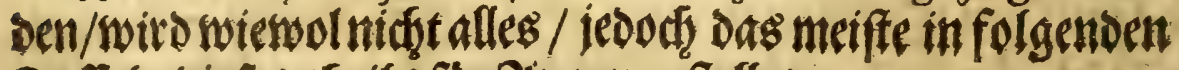
zaffern diefes theilg für 2iugen geftellet.

TABUT. 


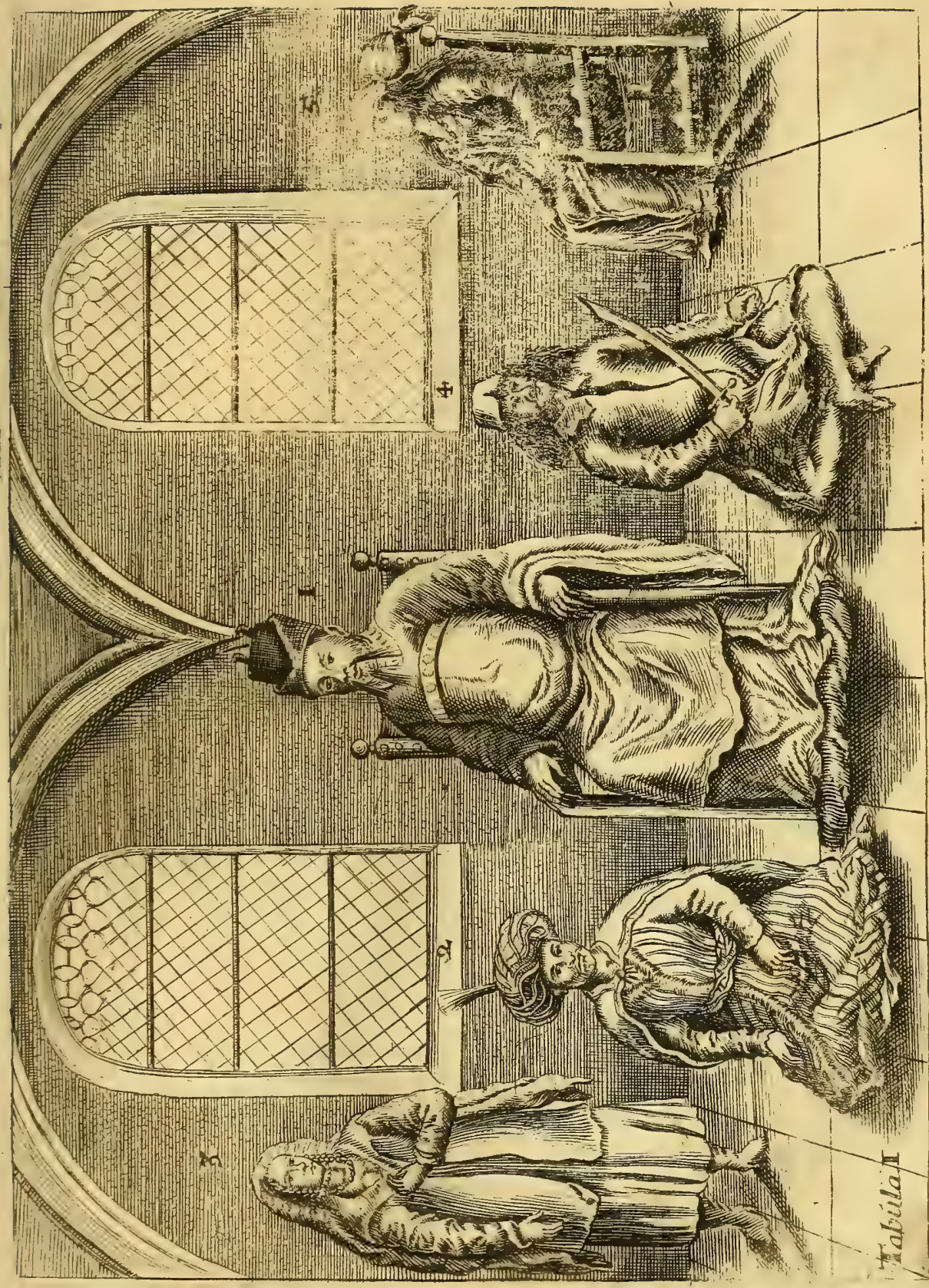





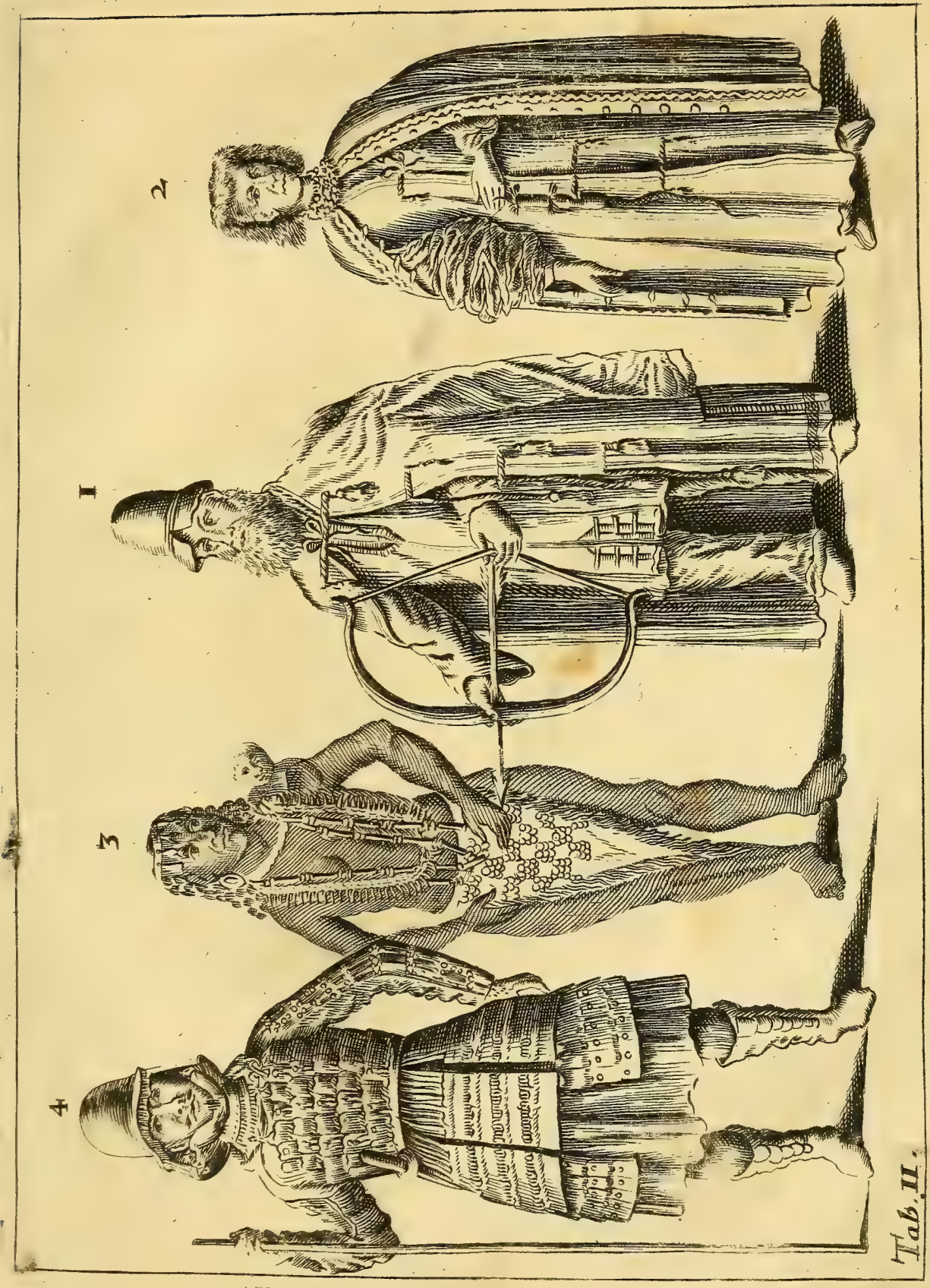





\section{TABULA I.}

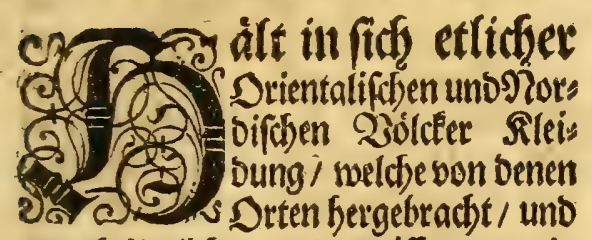
sum tbeil etlicben aus gewiffer materia gemaoften Silbetn / fo meift ifbe $\mathfrak{B}$ wegung baben / angetban fevno.

Unter Den Sleibern fery Satubbe aus๋ Seebumbes Darmen gemadjet/ uno Rócte bon Seefunbes Fállen. 2lud etliche son 23 aft gar Eunflid) ge wircte Sleibet / Suffen uno Sapeten Dieson ferne anjufeben / aud fo roeid antugreiffen / als twennz Setoe uno Sammit roaie. Soldestrteit toiro in Angola gettacht / uno it uns aus Guinea zugefidict worben.

Num. I. Sftein SEinefer/obet nad unfer pronunciation ein Tzinefifdeet Serz/m feiner (Seftalt uno Habit nad) Der Chimefifden Mahlerey / fo aud) Darben verbanden/ahgebilbet.

2Auff folde 2tre / mie Diefer fitget / ftels lenfie aud bie Biloniffe ibrer getwefes nen Şer2\} fchafft uno Sanbvoigte/ wenn fie wol regieret/ und Dem Eande groffen

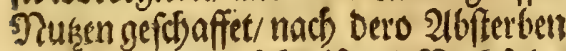
sume Ehren=(Sebdichtnis und Nach folge Der ansern incinen Dargu erbaueten

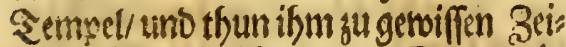
ten Soottliche Egre als. Darboir in

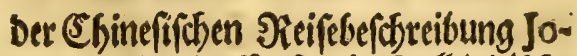

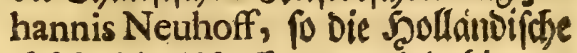
Dițnoianifthe Compagnie Dabin ges than/mit mebzem gulefen.

Num. 2. Ein Perfianer/nads ibre:

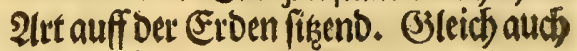
Der foúnig ben Befandeten Audientz su ettheilen pfleget. Darbon Olearii

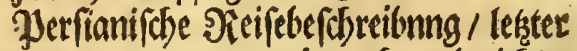
edition pag. 509. ein neefres beridbtet wirb.

Num. 3. Eine \$erfianiche $23 e$ e bezs:\$erfon in ibrem Bierath/ Die/ weent fie nicht gemeine Şuren/ ithe 21ngefictst

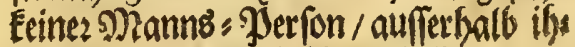
rem Ebemann/entblófien Durffen.

Num. 4. Ein TagettbaniftherDies bilcher Tartar / fo im उsebirge an ber Eappiphen See wobnen. You wele

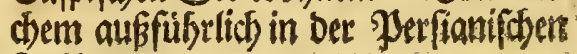
Fitife pag.725.

Num.5. Eine cirtafilithe rarta rinne/ gleich bie 233 itwen getleidet ges ben. Scaben binten am Sopffe eine Sinbes:B Blafe mit buntene attun úber fogen. ibid. p.742.

\section{TABULA II.}

Num. I. Ein \$oforwiter/an wels d)en su fehen Dertoleicher iffe fremboe/ Utberrócf und Schapfe ober Sommers

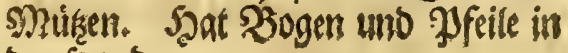
Der şand.

शi ij

Num, 
Num, 2. Fine Mofcomitif be Staue/ Derer Scembde Ermel / fo fie Des Sommers burch Den Siock fefen laf́ fen in 8. oder 10. Ellen lang/zufammen geftreiffet. Je bornefmer fie fenno / ie lâinger bie Ermel fern muiffen. Scatei ne Sinutpeitfdye in Der Şand/meld be Der Water iftem Siraiutigam nach Der Socbjeit zum (Gjeforfam feines 2 sei bes mitgeben foll. $23 a s$ von Den Sd)lágen / fo barmit aus siebe gegen Der Frauen gejchehen / gu balten / if in Der Mrofeowitichen Mieifebefchreibung p.217. 8u lefen.

Num. 3. Eine 2 seft Inbianifore Frau aus Mexica, wie fie Die Eleinen Sinber ju tragen pflegen / fampt ifreni Bierath/ welche von Bábnen uno Silaus en etticher wilben SGiere an fitatt Der súlbenen Setten. Darbon Johan de Laet. in Hift. Americana julefen.

Num. 4. Ein Sharnifd und 2 Baf: fen aus ber reichen Sft Jno. Jnfel Zeilon, aus melcher ber Zimmet ober $6 a$ nel haiufig isu uns fómmt. Sintemabl Dafelbft gantęe Bimmet:20álber anges trofien werben. Vide Mandels̈lo Sn Dian. Oeifechefdreib.p.147. uno in ben Sollánoifden Schiffafiten. Uno bat Diefer/fo bas 2 Baffen trägt / einen Canel obel Simmet: Stoce in Der Sand. Es bat aber nicht Das gankse Solk / fonbern nut bie Sinoe foldbe Aromatipche Sirafit.

\section{T ABULA III.}

Num. 1. 2.3. Senno Der Birtin lánber rashaffige Conterfeite fampt ibrer felzzamen sracht. Die lebenoige Werfonen batte S. STon. Majelt. ju Dennemarcf auf JetrnSceintich) ?úl lers I jerço Sentmeifters Schiffe aus Gitunlano betommen / uno zu unz nach (S)ottorff/ felbige anju/doauen / gefdis stet. Was ibre ? latur/Sitten/Spras be und melye Dencfwurbiges von ifs. nen zu fagen : wie id) an ifinen/ in Dem id) fie fuilf Sage in meinem şaufe ges babt/ meift felbit angemercfet / ift in Det Jerf. Sieifebeids. pag. I 63. unt folgen Den 2 láttern ju finoen.

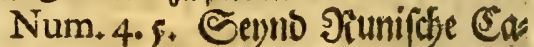
lenoer / Derer fich nicht allein bie alten Sotben und Dánen getrauchet / fon Dern aud) Die Raplánber fith nod ges brauchen follen. Won foldben Calens Dern if Olaus Worm lib. 2. Faftor. Danic. und in Deffelben Mufro pag. 367. julceen.

Num, 6. Ein aubgebauen Sthiffs lein oder Schlitten/fo die Eaplánder unb andere Norbifhe Woilcker auff Dem Sdinee gebraudsen.

Num.7. Seyno ibre / wie auct bet Finnen Sdyrit: Sdyube theils 6. theils 8. Fus lang / mit welchen fie auff Dem Sdjnee geffywinde forttommen toin nen. Sierten ift auch ein sapländifcher Iock von Rentbierbifiellen gemachet/ 


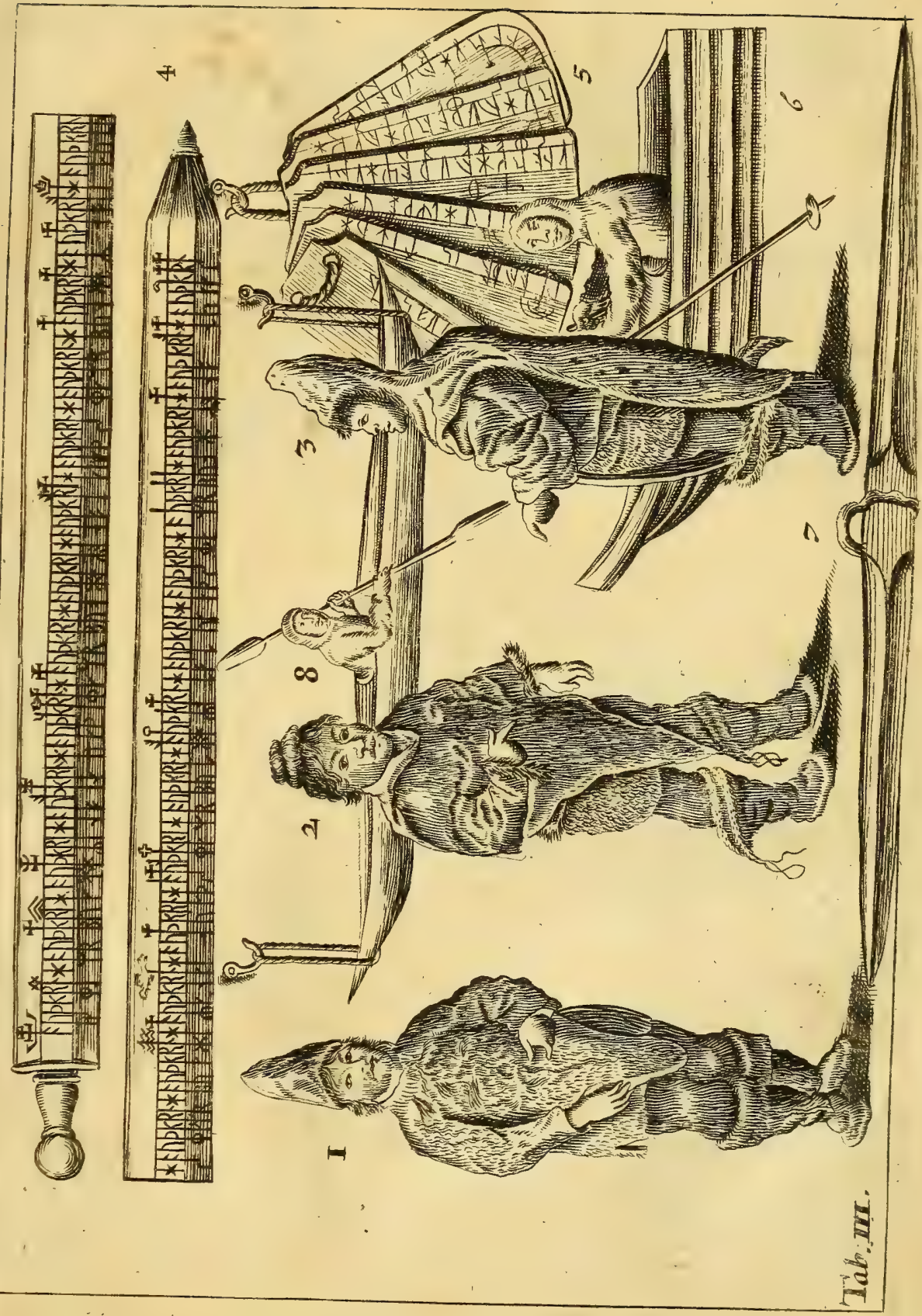





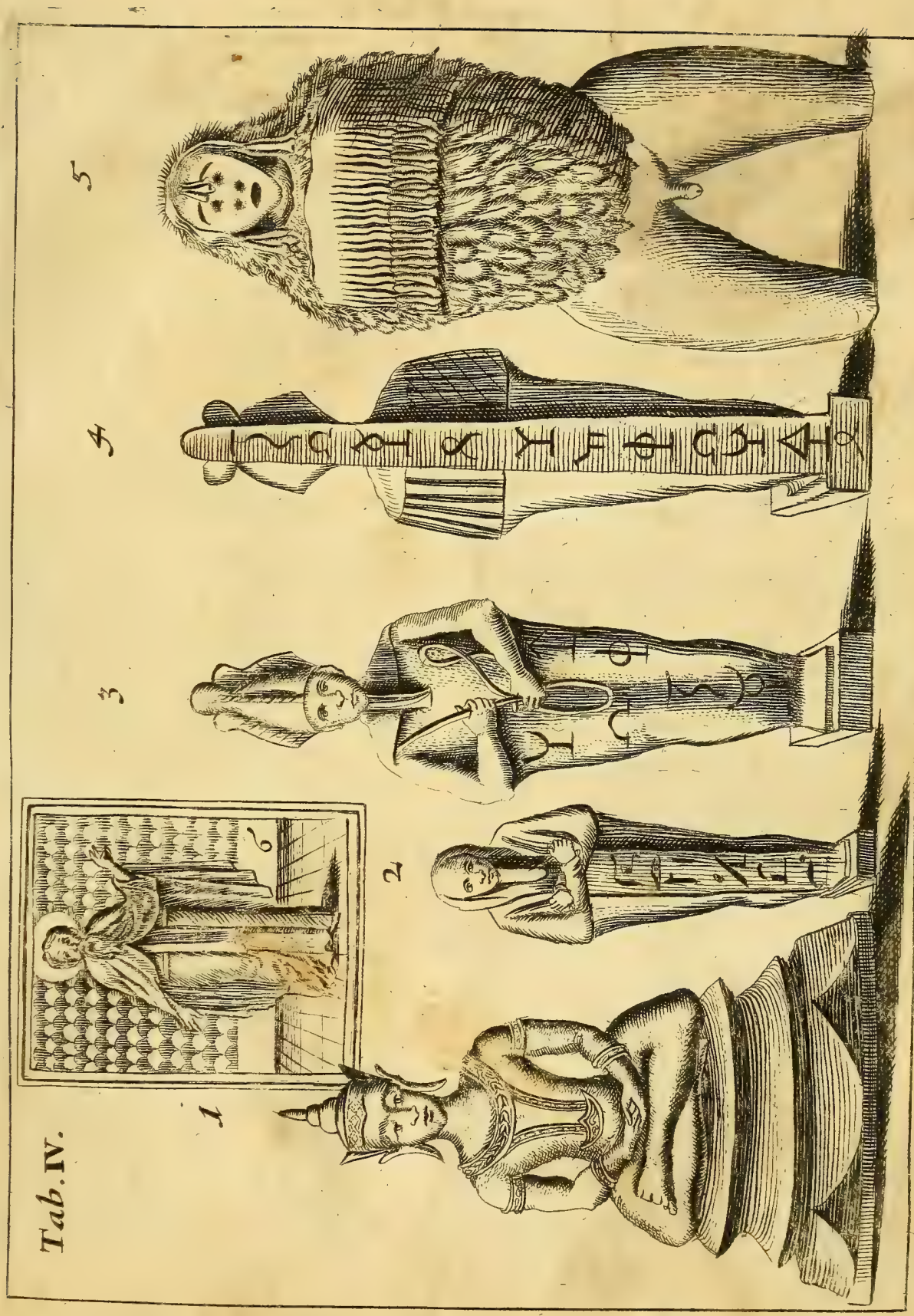



unb bon ibnen mit gefponnen Bibn (gleich man bey uns mitSiller pfleget) Befticlet.

Num. 8. Sitet ein (3) runtänoer im Schiffe / foumb den \&eib und Naund: loch Deg @ Chiffes zugebunden / mit wels chen fie bey ro. Mneilen in bie See fabo ren/fich bi isweilen umb und umb (t) swin: gen Eónner. Shre Siuber feyno gema chet/Daß́fie an beysen Enden Eónnen ges braucbet werden.

\section{TABULA IV.}

\section{Seyno lauter 2ibgôtter.}

Num. I. S住 ein Snoianifber Yagobe / Der von etlichen will fúr ein 2leguptifor Ifis angefehen werben. Das bon Kircherus in feinem Oedipo EEgyptiaco de Sinenfium \& Japonorum Idololatria.

Num. 2. Ein 2lbgott , Horus ges nant / weldhen man in ben 2reguptifchen Mumien finbet. Darbon aud Kircherus pag. 215. aus Dem Heliodoro lib. 3. Etthiop: und meynet e8̊ aus ben Siabbinen zu ermeifen / Dak Eabans Bjobse/ welden bie ?iabel ihm geftolem/ foicher 2art foll getwefenfenn. Olaus W orm meynet / Daß́ $\mathrm{eB}$ Icdola Ifidis Egyptica fino. Vide in ejus Mufro p. 348.

Num.3.4. Ein 2leguptiffjer $\mathfrak{A} 6$ = gott voller Characteren, wie er forn unb binten ansufiben.
Num. 5. Sit ein 2lbgott Der Norbs lánber ben der Strate Davis, umb wels doen fie/ wie bie Eiruinlánder/Denen ich es gejeiget/berichteten/ berumb tanken. Sit betleidet mit rauchem Schaafferll Jogelfeoern/ uno Eleinen Zábnen bon Sifdien befjangen. Dannfie meynen/ weil fie von Den brev Elementen ibre Nafrung baben / muifen felbige aud als (Siotter geebret werben : wie noch jefro Die Scenden im Sionigreich Siam in Sf: Snoien thun/fo die Elemente eh: ren / uno ibre Şegrábniffe in benfellen erweblen. Die Das Teul geefret/wok Ien verbrandt/Die Daz'2 Baffer/audb Dar: innen ver/enclet/Die/ fo Die \&ufft geefret/ Darinnen auffgebenget unb von Jogeln gefreffen feryn/ uno andere in bie (EvDe/ fo oieflibe geelyret/ verfocharze

Num. 6. Sft cin Nofentitich SBild/S. Nicolai, mie gadb etliche ants Dere Jeiligen. Sold gemablte Scei ligen mus feglidser in feiner Stuben uno Siammer baben/ uno vor Denfellen ftes beno beten / uno fich franen uno gar offt neigen. Und wemi jemand zu ifren inş Şauf fommt / mus er juoor ebe er jemano zurebet/vor foldsem Dilbe feine devotion thun / mito als ein (Sjott ges ehret/mie er audb) Buch, ein SSott genens net wil'b / uno muf von niemano als bon ifyer (s)laubens genofien gemabiet werber/ fefien aus / of fie fobon gans neue / als wenn fie cin Jabs im Siauche gebangen. SSerben alfo auff einen

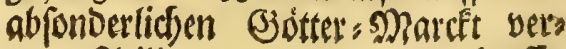
(iii 
Eauffitodernur / mie fiereden / umbs Bjelb vertaufchet / uno wab ber Siramer forbert/mufi der Siáuffer geben. Dats

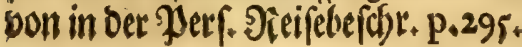

\section{TABULA V.}

Num, 1.2.3.4. Senno Silber/ roelche Durch gemahtite Fruidhte bie Wier Beiten bes Sabro albilden.

Num. $5 \cdot 6 \cdot 7 \cdot 8$. Seynovonnaturts licjen Saamen und Frtichtern/ fo in Den vier Sabres : 3eiten wachfen und reiff werben / fúnfltids sufammen geferet. Eo audb bie vier Jafroz: Beiten andeus ten follen.

\section{TABULA VI.}

Num. I. Yvana faft an Sieftalt eis ner groffen Eiber/fabeuslid) artsureben/ in gank America gar gemeine / bie Sirafilianer netmenes Senembi, fenno vom Ropffbís ü Ende bes Stymans Geg 2.3.in 5. Fuß lang. Etlliche haben unter Dem Salfe sinen Sropff / ettiche auch nicht/ wie wir bann bon benderley 2lrthaben / bat eine gar foharffe 2ifches farben Săut / forl menn fie leben / grins licjt teyn. TSirs aus Eleinen als Sirs fden:grofien Evern gejeuget/ balten fich bismeilen im 23 affer / uno bisweilen auff Dem \&anbe uno SBáumen auff. Daber Clufius ex Oviedo zmeiffelt/ Db es unter Die Sifthe ober Fleifक ju rechnen. Eollen úber brey \$o sot ohs ne Eflen uno Srincten leben lónnen mie Marckgravius lib, 6. rerum natur: cap. II ez feltbft probiret. 9 ghe

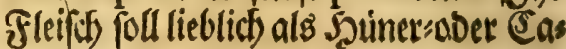
ninichen fleifa ju efien fern/ uno wiro bon Denen / Dic es eimmabl gefflymecfet/ fefre begethret. Dis sthier foll zwein Magen haben/ in Dent forberfern nimpt exerftlich gleid) als in einem Rropf Die Speife/ welche Durch cinen Darmen/10 ein Elein Finger bicke/unb bey sehen Fin get lang in ben anbern Magen/ Da bie Serbauung gefobiehet/gehet. Es forl gar ein fart Seben baben/wiro mitStris cken gefangen.

Num. 2. Ignavus, Diefes Shiets lein/Derer wir stwen haben/ifteonङreofs fe eines Fud) fes/ von Şaaren raudj und

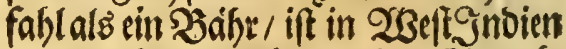
gar gemein/ uns rwiro von Den Eillwobs nern Ai, yon Den Portugie en Priguiza von Den Szollánoern aber: Luyart Fauls beit genant / foll einen ganken ₹ag/ neenn eb im ङange ift/ nicht úber so. Sobritte fortgefen / Ereucht auff bie S̉áume / friffet nur Das \&aub Darauff uno trincfetet gar nidsts/ fod / wie George Miarcfigraffi ber eó lebenbig gebaltunt anatomitet (Hiftor. rerum natur. Brafil, lib.6.) faget / fotreyen wie eine

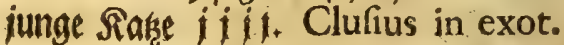
p. III. und aus ifm Athan. Kircherus meynen/ base es foll glcich nad) Der Mufic Die. Thone la fol fa mi re ut 

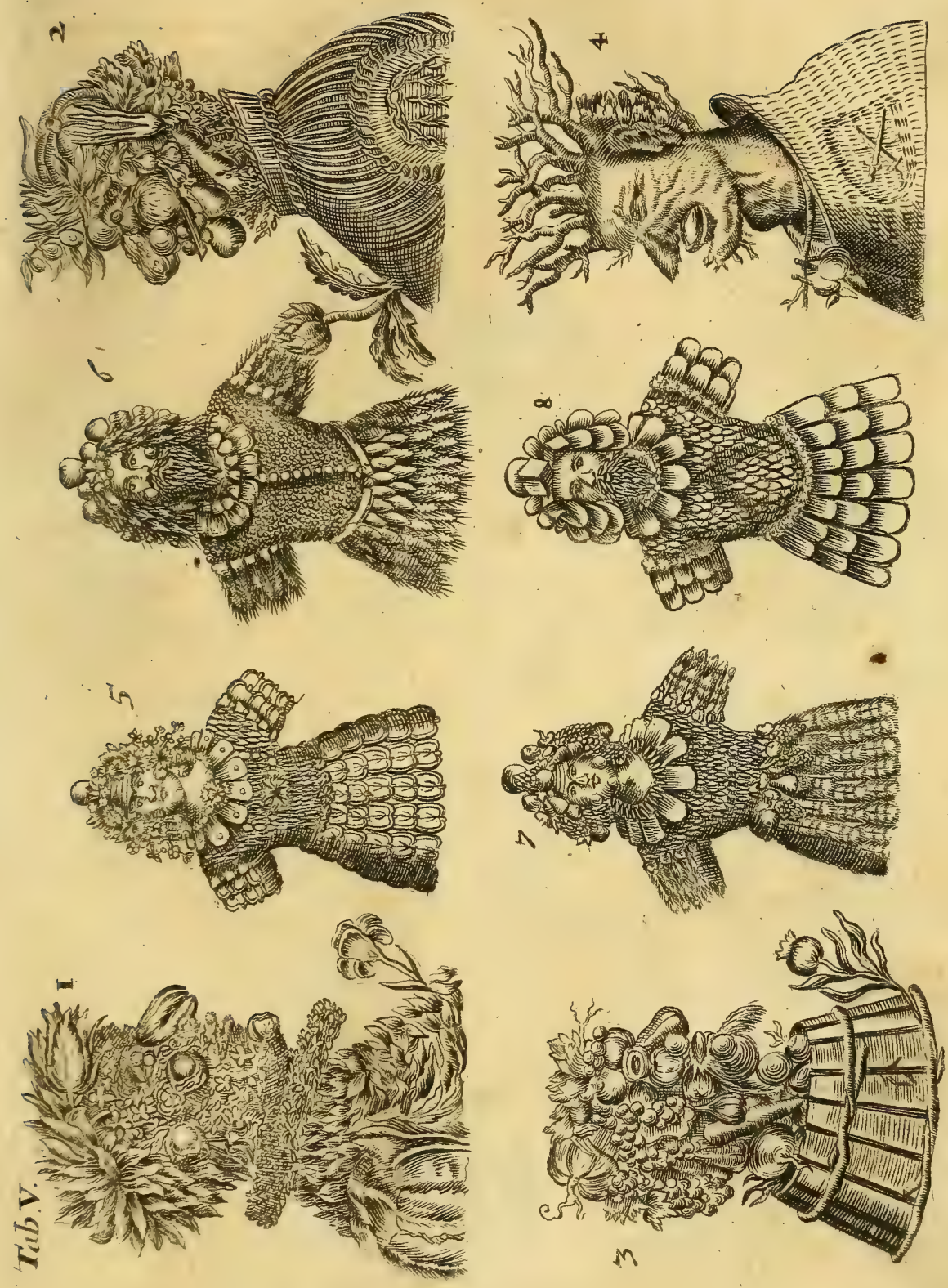



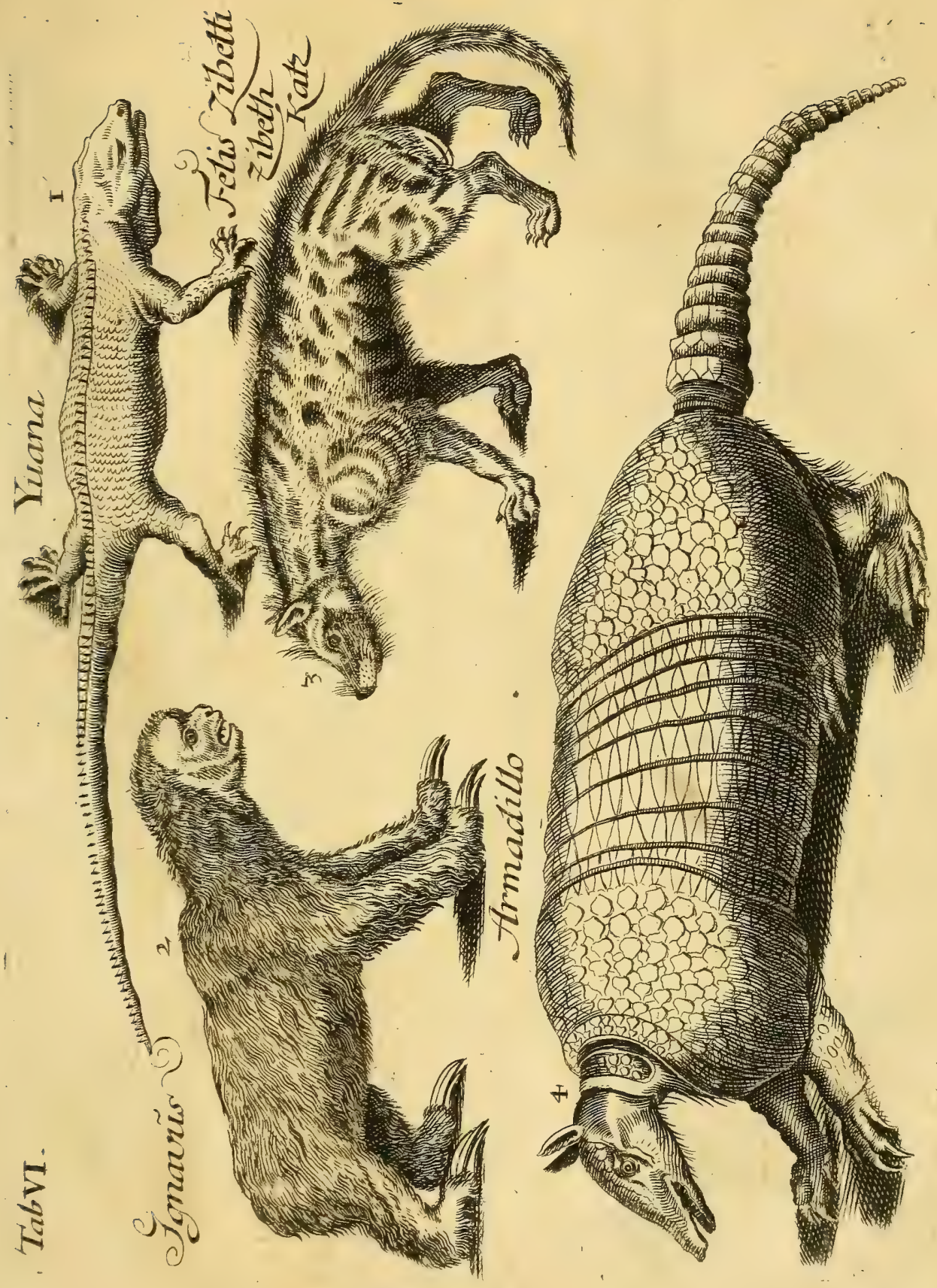



sitoentlid berunter fingen mit ha ha \&c. Ulno menn an benen Orten bie Mufic twaire erfunben worden / folte

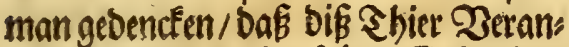
Iaffung barz̧ gegeben hätte. Es hat lan

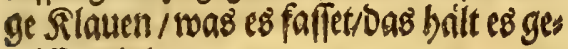
miffe und lange.

Num. 3. Bibeth=: Safge/ Derfelben bas ten wir auff Esottorff 6. Zetendige ges babt/ uno úber Sabr uno Sag erbalten/ nurben aus Guinea von Capo Corfo gefchicfet. Cardanus forreibet/ Dap fie niemable fónnen zabm gemachet werben. Es war aber eine unter Den fechfen/ fo noch etwas jung / weldse bey Dem Mienichen / Der fie wartete / alle Nachs friedlich im Bette fohtieff / und soenn man fie nufferfallb Derm Saften bló̈ gehen ließß / frriche fie fich als eine zafme Sauks Satge an bie Sbeine / als fie aber álter wurbe / begunte fie audis/ menn man fie angreiffen wolte/ umb fich zu beiffen/ uno rourde end lich fo willo als Die anoern. Trselches bem Cardano; wiber Den Scaligerum, ber ifn Exerc.

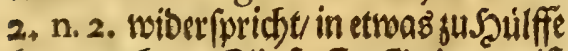
Fommen Ean. Sie freffen Brutuste/ weís Brob mit Mild aud rohe Ever. Sie baben unter Dem Scinterften über Den Pudendis einfohlammidjt faft als ein

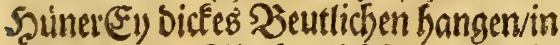

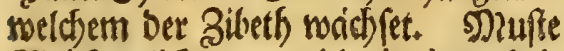
2Bóctentlic) stweymabl mit einem Eleis nen Zinnen feffel als eime Surrctifure Soone gros mit groffem smang alges nommen werben / fon/t feteidsenfie es felbft aus an bie 2 Bainde.

Num. 4. Armadillo, Difî shiers

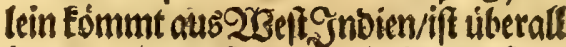
in America usu finden. Die Sirafilia ner nennen es Tatu, bie Spanier Ar. madillo, weil es als getwaffnet anjupes ben/ bat auds fo barte. Saut / Daßs man fie mit Dem Jfeile nicht Durchjobieffen forl. Eein gangerer \&eib ift gar jierlich als mit einem Sarnif getrapnet. Die Şollánder nenten es Schilb: Werctem/ Dann esan (Seftalt einem jurgen Wer" déen gar ehnlich. Es: bält fich gemeis niglich in ber Erben auff/wie bieझhauls wurffe ober Caninichen/ unb thun wes gen ibres lumbruiflens uno 2uffiver fs fens in Den Buorten grufien Schaden. Sie merben nie bie Eaninichen gefar gen/mit $\mathfrak{B u t t e r}$ gefoefet und gebraten/ follen ben Sellinineern fo delicat als Caninichen fismecfen. Sirafft uno 2Birtfung berer Schalen und Bsebeine ift berm Marckgravio lib.6. cap.8. refen.

\section{IABULA VII.}

Nüm. I. Lacertus peregrinus fqamofus, ift bom Sopff bi sum Schwange úber anderthatt fu 1 bex Sdjwank aber fo gar breitein Tup und 2. 3oull lang bie circumferentz Deg śé 6eg I. Jैu 3. 3014. Die Sthuppen feyno Soll breit und bart ate scorm / lies 
gen úbet einander / als toie man an ben Durzen Danapffeln fiebet. Şat aud folde braungelef farbe. Clufus befótrei bertibnaufúbrlich pag.374.

Num. 2. Iguana it oroben Tab. priori befdrieben. Slur Dif oarben al ezinneren/Daf fo delicat DeffenFleifód su effen/ fo fobáblid foll es Denen feyn/ toelde an morbo gallico laboritet baben/ ob fie fojon lainglt Darbon gene: fen/foll es Dod die Sdimerisen wieber serneuen. Cluf exot, p. 117 .

Num.3. Erocodill wito aus Evern gezeuget/ Deret eines Darbey bánget / ift unfert Scuners Sisern gleich an Siroffe, mut Dafies nidst oben fo fpis su/ fonoern auff Cylindrifdse Altt frillt. Diefe Sbies rewerben in gnoien/ uno am meiften am Flus 2rilo in Xlegnpten gefunben. Senno Den simwobnern an Shenfoden uno Qiebe bódbftidáblich. Diefes un ter allen Iffieten bat Eeine Zunge. Plin. 1. 8. c.25. S8 verfolget bie/ fo fur ifm flieben / uno fieudyt fur bie fo ifm nadj: cilen / ift geldswinbe im sauffen / aber wie man ifm entfommen fan / ift in פ) Ranoelölo Gnbian. Sieifebefóreib. p. 67. ju lefen. Jonfton. de quadruped. fojreibet/Dafin in America bev Det Stabt Panama etlidbe von 100. Fuf lang follen gefundern werben.

$$
\text { TABULA VIII. }
$$

Die vier Cbierlein in viefer Tabula follen bie vier Elementa bebenten.

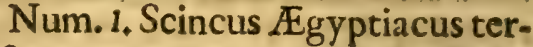
reftris \& montanus, foll nur auf ours rent Eroveiós leben Eónnen/ uno bebeus tet bie Froe. Somftift aud ein ande: Aquaticus genanot/ fo fich im 2 (Baffet befindet/1wiro in Africa bey Lybia uno am Sothen Sreer gefunben / ift ben Apotbetern wol betanot/oie ber Spras d)e unerfabren ibn Stinc nennen. Diofcorides nennet biefen Crocodilem terreftrem, und faget / Daf er certis vehiculis fumtus vim ciendæ Veneris vehementem babe/auc $\$$ intenfam illam Veneris cupidinem inhibite. lib, 2. cap, 7f \& Gesnerus lib, 2. cap.24.

Num. 2. Acus marina (sine?)let: natel 1 ift ein gat fontmalet vierecter Fifd/ber/ fo balbet aus Dem 2Baffer fommt / ftirbet/ beocutet bas 2 Saffer. 2 Sito befdrteben boinAriftot. Oppia. no uno Bellonio.

Num. 3. Chamaleon bebeutet bic sufft/ift ein A fatifos Sbierlein / febt: mager/ foll ben ganken seib boll sunge baben/mie Gesnerus aus bem Plinio lib. 8. cap.23. beridstet/ baber mennen fie/ Daß̧ešoun ber \&uft lebe. Agricola. Aber weil es eine lange formale sunge als einen bicken 3mirns fa den hat/fo gat fofleimicht/ forl es feibige heraus ftecten/ Mucten uno Fliegen barmit fangen. Scal. de fubtil. Exerc. 96. 4. 23el dem ßeyfall zu geben/ fo bat man fele biges S Gierlein yor 40. Sjabren allbier 


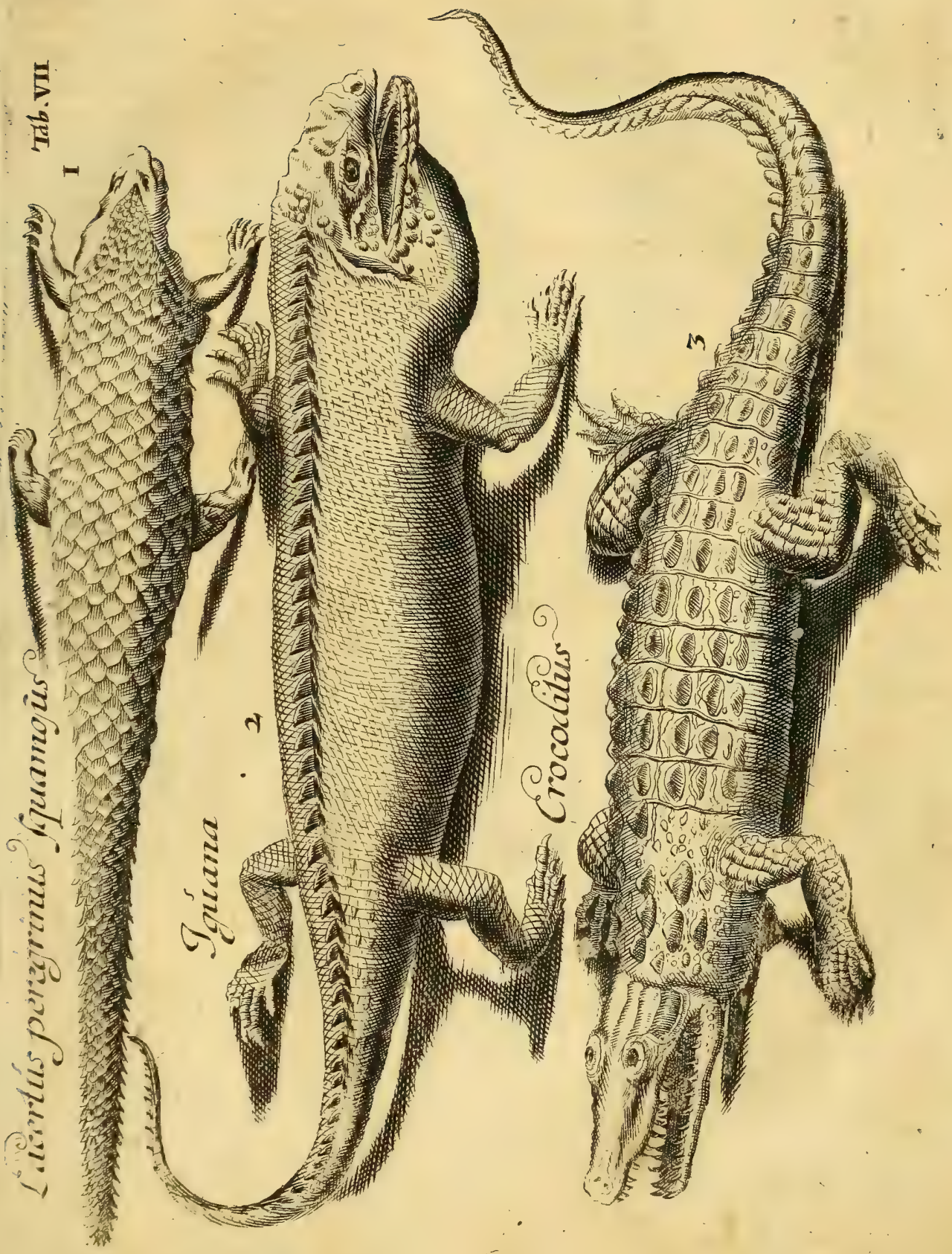





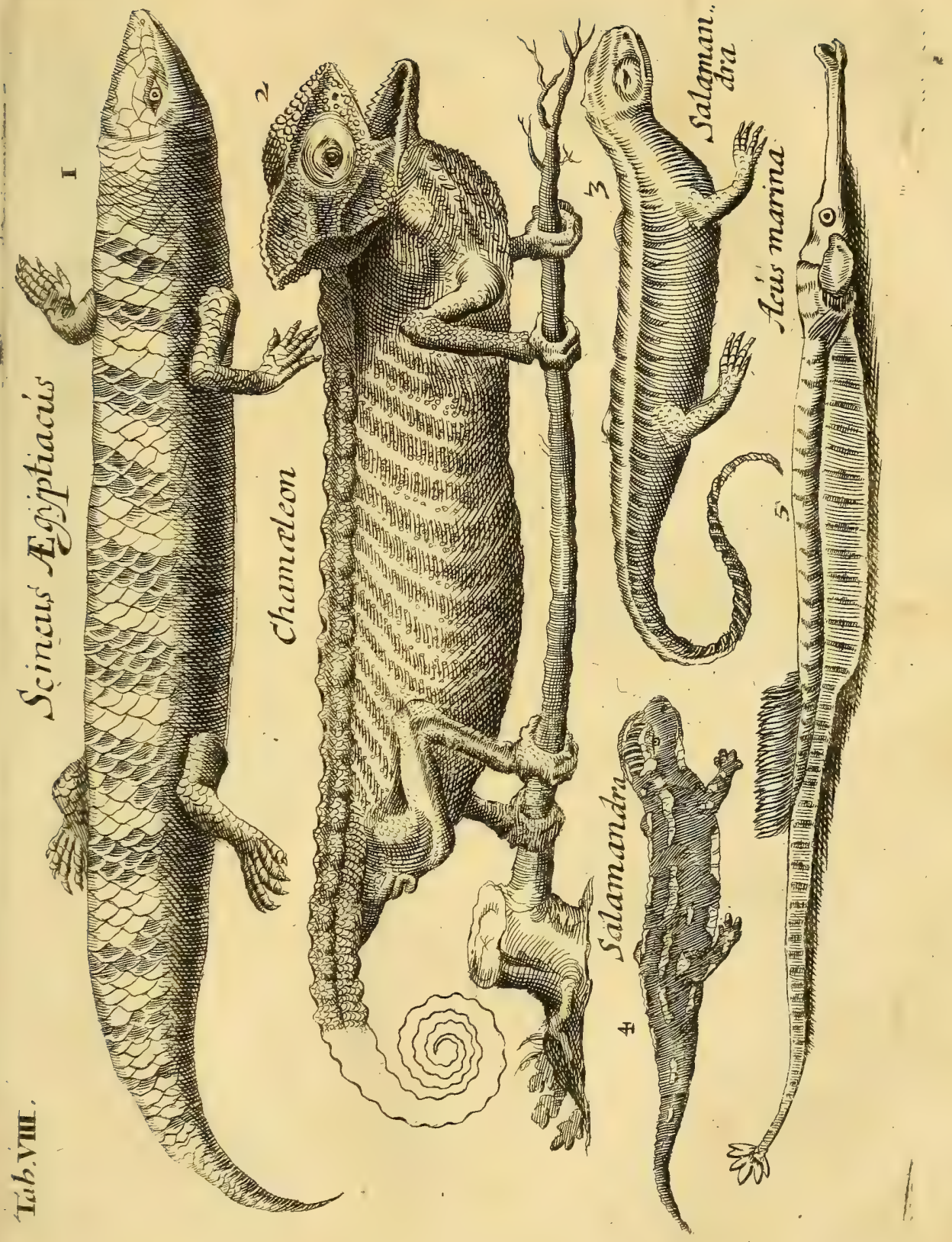




3ิu (3)ottorff lebendig gefsabt / und nach Dem es geftorben auffgefobnitten / uno eine STliege im Magen gefunden. Und soil ç Daza allerfurdht;am/te Shier/ foll es Daher fo offt an Farben / auff welche ez fitset / fich verainoern. Plin. lib 8. Daher Das Sprichwort : Chamæleonte mutabilior.

Num. 4. Salamandra bebeutet Das feur / fo ferne auch ein Elementum Ignis ift. Es ift eine 2(rtwon Eiberen/ bailt fich an feuchten Dertern auff. Plinius lib. IO. cap. 67. fchreibet / Daf bet Salamander wegen feiner heffitigen Siáls te audb Das Feur auflefichen fod / gleich swie Das Eis thut. 2atber id balte/Da eв wahr fen/ roaz Gesnerus aus Dem Theophrafto Durd) Erfahtung berich: tet ( pag.83.) Dafís er einen Salamander

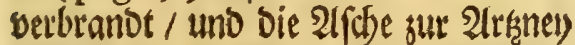
gebrauchet. Wrsegen Des beffitigen

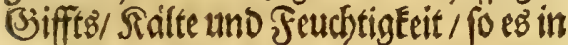
und an fich) hat/ mag es nool / soie Seneca faget (lib.19, Ep.9.) mitten Durchs Teur unber fefreet lauffen / auch wol eine sweile auff gluende Soblen und im Feur Dautren Eónnen / aber muş Doch endolich verbrennen / mie audj beffen Ever / alB Scal.Exerc, 185, uno Agricola de anim. fubt, berichten.

\section{TABULA IX.}

Num. 1. Sft ein Einforn/ 10 8. Tuf 4. Boll lang ift / hey weldsem nods glwen Eleinere. fich befinden son fünfftes

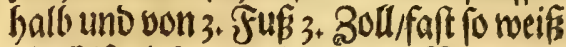
als Elfenbein/und penno won Natur ges Drefhet. Dak aber foldbe Shórner fol. ten von einem vierfúfingem Stbiere pern fo man Einformer nennet / in Brooffe und Bieftalt eines jungen $P$ ferdes/ $/ 0$ in Den Srientalifosen \&andern in Den WSildoniffen fich auffialten follen / wie - Die 2ltten Datbon gefdrieben / ift nidst

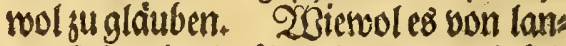
gen Zeiten ber Dafúr gehalten / uno for che sadrner von vormefmen Saerzn uno Jotentaten theur an fich geEaufft / uno als ein úberaus Éóftlicber Schak vers wabtet morben.

EE wirb difputiret / ob auch in ber 2rselt foldbe Shiere / nemblich Einfór ner/ zu finden oder getwe fen feynd / weil zu unfer Zeit/ Da bie 23elt bods siemlid Durchgerwandert/ Eeines bon jemanto ges fefen/und oavon 2jericht gethan not: Den. Bjleichnol aber finder man in

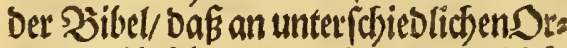
ten ber Einforner gedacht / unb (3)leidj: niffe Darbon genommen worden. 2H:

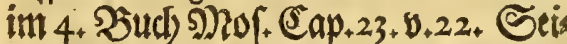
ne Sreubigkeit ift wie eines Einhorng.

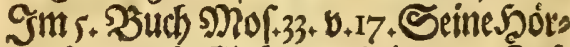

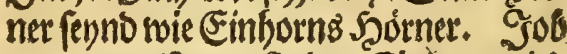
39, ט.12, Necyneftu Das Eintorn wiro Dir bienen/uno merbe Gleiben an Deiner Rrippen. Paalm 22, v. 22, Errette inich von den Einfortnern. $\mathfrak{P}$ falm 92 V.II. Mein Shorn wirb erfod bet / wie eis $\mathfrak{3}$

ner 
nes Sinbornz. 2ber eв ift qu miffen Dafin Sebreifonen und (Sirumbtexte als

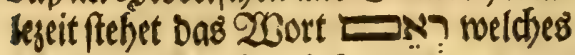
nicht eigentlid ein sinforn Ünicornu, ober Monoceros (mie es Die 70.(Stries d)ifche. Dolmetidser uno aus ibnen alle anbere Sprad)en gegeben ) betffe / fons Dern ein farcfes / wildes / fredbes mit boben Soirnern begabtes/ Den alten She brxern wol betanbtes/ uns abet ( wie Marinus in Lexico Hebr, faget ) un befandtes Shier fey. Bu bem will auch aus Dem 33. Sap. Des 5. Wud Mofer: bellen + Dabifif Shier Reem nicht nut ein / fonbern stoen Sornet gebabt.

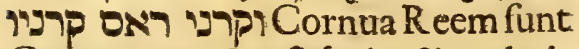
Cornua ejus Da fefet ber'Singularis $\square \times$, nicht Det Pluralis Die Soorner eines Reem. Uno weil Der Dualis "gapl ftebet/ werben Gofephs stoene Sobne uno Stamme Sphraim uno grannffe Darmit befótrieben. (5: binbert auch nicht / Daßim 92. Plalm febet/mein Sorn witi erbóbet / wie et: nes Finborns. Gm Sirumbtexte ftes bet exaltabis cornu meum, ficut Reem. Du wirft mein Sorm aufiridy: ten/ empor betien / wie Das Reem ju thun pfleget / Doer wie Deb Reem Saor: ner erfobetfeben. Uno Rab.Eliezer (wie Der gelebrte Bochartus angemer: cfet) uber biefen Locum fortreibet: quid iष reem? Altiora funt cornua ejus quam beftiæ cujuslibet, \& ferit cornu tam finiltro quàm dextro cignet er bem Reem audy smey Soirner 3u. Darumb beiftes nicht Einborn.

Orix vel Capra Sylveitris, cine?let von wilben Biegen, Davon Plin. I. II. c. 46. Diefer foll fehr graufam uno ftarc $\mathrm{f} /$ uno wenn Dem Herodoto (1.4. c. 192.) su sláuben / fo grop als ein Ddbfe feyn. Uno baf biefes Durd Reem verftanden wertoe/ will Bochartus de animalibus S. Scripturæ be baupten. Deffen Urfacben bier einzus: fübren/ zu weitlauffig fallen wolte.

Utrfac) aber / warumb die 70. (3rties: chiffre Dolmetfcher Das Reem einen Monocerotem obet Einborn berbols met/chet/ Ean fenn; Daf fie gefeben / wie Ctefias ein Lifralter Siriectifoter Scribent (Der zur Zeit Cyri, fo mit Dem Artaxerxi Riricg gefübret) einen Onagrum doer Snoianifben wilben OBalD Efel/befchrieben mit eben folden Ümftánderm/ als Dem Reem in Det $\mathfrak{S}$. Sd)rifft zugeeignet wiro / Dab es ein ftreithares Shiel / fo mit bem Scorn Bábnen uno Fuffen feinell Derfolgern auffig graufamfte fich erseige/ fonberlich/ Dak er an Der Stim ein Scorn baben foll: und Den Spruch Davios im 92. DY falm v.7. Dargegen baltenoe / Gaben fie gemeunet / Dab múfe bielleidyt ein fold Stier fern/ und weil fie Dod) nods

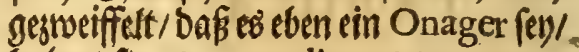
baben fie es generaliter Monocerotem Einborn genennet.

Sonft finderman audf bey dert ats ters 
ten unb theils neuen Shiftorienforei bern/Daf unterfobiebliobe Ibiere/fo nut einsorn baben follen. I. AlB Da ilt nicht nut bet iekt gebad te Onager ober milber 2 Galb=Sfel in Snbien / Darbon Phocius in fragmentis Ctefia Alianus lib. 4. cap. 52. Mlin. 1.7. c,21. Sonbern auch 2. Equus Indicus $Æ$ lian. lib.3. cap.41. 3. 2Silbe Ddben in Snoien. Plin. ex Ctefio. Julius Cafar lib. 6. Comment. befphieibet audbein Sbier / fo ein Sorn an bet Etirt/ und am Sarks (e1) gefeben wors Den/ Daroon balo folgen foll. 4. Paulus Venetus gebencfet 1.3. c. I5. Dap in Java minore gefeben worben ein Sbiet mit einem bicfen fobmarigen Sorn an ber Stitn / welifes/ wie bie Sdbrueine/ fich gerne in sotbe auffbalten foll. Vartomannus (Deffen relation Scaliger Exerc. 205. gloffen Bjlauben zus ftellet) faget lib, I. Navigat, c.19, Daf

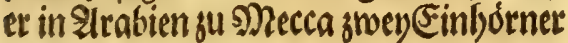
geleben / fo neben bem Sempel in einem Gatter betroabret gegangen/Das eine itt

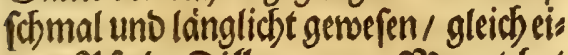
nem Y Perbe'Futllen bon 30. Monat/bat an ber Stirn ein Soorn brey Slllenbogen lang gebabt/ bes anbern Sorn aber nur vier Sano body/ Der Ropffif faft einem Sirfó gleid / Der Mabilgar furs nur auffeine Seite bangens getwejen/foblan:

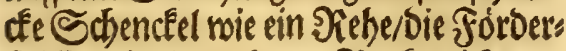
Fuffe gefpalten/ einem Sodfe nidyt un

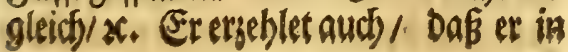

Zeila eine Stabt in Ethiopia eine Siube geeferen fabe / fo ein Sorn am Ropffe uber eine Şano hod) / weld)es fich gegen bem Situcten geÉefret ges babt.

Unfer fo langeb Syorn aber wie aud Dabju S. Denis in franctieid / unb Die beuben fo gu Utrecht gefejen werben feyno leines oon obgedacten shieren fondern von einer fonberlichen Art Wallfifonen ein Sdjnabel / ober viel. mefre ein berausftebenoer Babn / fo in Der 2lorb:See/ fonberlich bey Siruins lano fich befinden. Daber die obges Dacteten (3ituinlaindifche 2 Beiber (p. 4.) Diefes Scorn/ als idhs ifnen geseiget/alb: balb getennet/ uno gefaget / Daṕp folche bey ibnen aus Der See Eommen. 20as biefes Şorns Sugent uno Wirctung betrifft/ fo fchreiben bie meiften er fabture Medici, Das es ein gewiffe Remedium wiost die Siifft uno giffige Sirand beis ten fey I gleich man von bem rechten Einborn ou balten pfleget. 2Biemol es D. Thomas Bartholinus in bet Jrobe nicht gar berwaibret will erfunsen baben/ wito er Dod joom Tulpio lib.4. obfervat. medic. wiberleget / uno ifm gefeiget/ wormit vielleicht in Der Yrobe mag geirzet fenn. Ert aber Tulpius for be Die Jrobe gut be funben. (sileich aud Olaus $200 \mathrm{rm} /$ wie er in feinem Mufro pag 286. herichtet/ und fúbret fats liche Teftimonia yon einem ganken Collegio Medicorum su 24ugfpurg $\mathfrak{3}$ ii

mik 
mit ein/Daf fie in Der Jrobe befunden/ Daf bergleicben Art Finforn miber bie siffit ein bewebrtes Alexipharmacum (ev).

Num. 2. Seyno Sơner bon éinem Sleno/ Alces, fo groffer umb ftarcfer als cin Sirfofs. Sch babe in Ingermans Jano uno Sarelen gefefen/bie an Siroffe ein ftarce bobes Jjerb úbertroffen / bes finben ficb gar báuftig in ben Norblain Dern/fonnen zabm gemadt werben/Daj fie ins Saus su trincfen fommen. St: lid)e fichreiben aus Dem Olao Magno, Dáp Dif Shiet (e lend genant werbe/toeil es tåglich die binfallende Sudbt baben foll. Uno menn eš mit Dem redjten Sinter: Fuf fic nur ein wenig ans Dht Fraket und fószammet/ foll eg roieber ges nefen. Sos tin aber von furnebmen Seuten / bey benen fie gai gemeine uno zabingeben / beridbret / Daf fid bs tid alfo verbalte. Geodeb ball man bas fur / Dap bie Elenobislauen febr gut sutber bie binfallende Sudbt fenn follery wenn man entweber Daraus gemadote Singe an Dem lincen (3) 15 : Finger traget / ober einem / oir mit foldber Stancfbeit bebaffet / ein Stuct baroon in die lincfe Şant leget / uno bie Sano zubrilt/ oder im lincen Dbt samit bers umb fábret uno rubret. Vid. Olaum Magn. lib. 18. c. 2. \& Jonfton. quadruped. pag, 97. qui addit hxc. Suffocatæ mulieri rafura cum Zedoaria anaticis partibus felicisfime exhi- betur. Won felbiger 2at Sieweifen baben wir brey paat / wie aud etliche Seine uno Túffe / Derer stween su reinces geffirzen gemadte feyno.

Num.3. Seyno Şoimer bon einee (3)ems/Rupicapra genant / fo fich im Schweikger:Srebitge bauffig finben las fen. Sragen bie Scoirner ructuerts ges beuget/ mit weld)en / wenn fie auffis euf ferfte berfolget werden / uno Eeine 2uss. flucht feben / an Die fteilen ferlen bant gen/ in Mesunung Dem Jager ju entrins nen / werben bod alfo vom Säget ets foboffer/ober múffen fic berunter ftuiro ken. Ea fónmen bie Derfolger Diefer Shiere/ Doer Siemsfteiger / Daniber in Rebenz:Sjefabl gerathen/wie folches Der bod)loblidje Rainfer Maximilianus Primus (ein fonderticher siebbaber folo cher Jagit) an fids erfabren / wicers in feinem Sheuroand felbft betennet pag. 16.1 7.19.52. 1tho Cufpinianus in vita Maximil. p.614. gebendet.

Num. 3+4.5. Sinno Rhinocer Scormet / Deren grofftes gar ungemein und 3. Fuf lang ift. Sold Scorn iff bon einem Sbiere / fo etwas fleinet als ein Elephant / uno bat bas Scorn forn auffoer ?afen feben / baber es audb Den Namen beformmen/ wiro in Jnoien angetroffen/fonderlic) umb Cambajas Bengala,Siam,Sumatra, Java major, wie aud in .thiopia uno America, wofelb/t ifte abgeroorffene Scoiner aufs gelefen uno su ung gebradbt werben, Difi 
Sbiet foll bem Ariftoteli uno anbern Siriechen/wie aud Den Siomern uno in ganf Europa unbetanot sewefen fety biş sut Zeit Deg Sánfers Augufti, wel cher / wie Dio Casfrus lib,5r. mennet/ sum erften mabl/ nach Dem et bie Eleos patram tibertounben/ sum sriumph in Siom eingefúbret. Plinius aber (toel

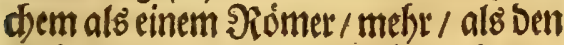
Biriecten (Silauben zusuftellen) lib. 8 . c.26. jaget: Daß $\mathrm{C}_{\mathrm{n}}$. Pompejus Magnus, Der viel Şabr vor bem 2tugufto gelebet/Der fery gerwefen / Der in Siom zum erften mabl einen Rhinocer ges bracht/ und stum Schaufpiel auffigefübs ret. Es if ein gar ftarcf uno grimmig

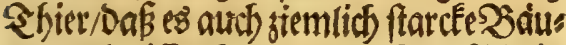
me umbreiffen Ean : roie Merctein in

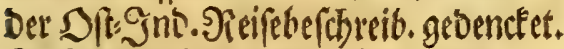
Daber $S$. Hieronymus in Comment. Jobi cap. 39. faft Der Mennung ift/ Daf Der Rhinocer Das Einfotn Doer Reem, fo (mie oben gemelbet) offt in J. Schrifft gebacht wird/ feyn muige. Es iftein grinmiger Feino Des Eles phanten/ ber fich wol fúrferen mus/oa Der R hinocer ifm mit Dem Scorn nicht unter ben Z3auch fómint. Uno rener zum Streit gehen will / wefset er Das Sorn ant Felien. ManEanan benew/ forwir haben / Fehen / Dabfie geftritten/ weil fie fharff uno die Steimiffe roch an fich baben.

Emanuel/ ber Foonig in Jortugal/

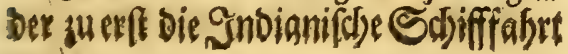

auff(ud)en laffer/ hat Anno Is I3. einen

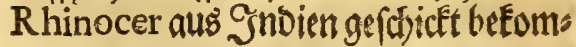
men (Surius p. 82.) und Anno Is 13。 in einem Sd)aufpiel mie einem Eles phanten ftreiten lafien: und hat nach lan: gem Biefecthe Der Rhinocer Den Eltes phanterterteget. Aldrovandus lib.I. quadrup. pag. 883. Demfelben Rhinocer bat 2llbrecht Dúrer/Der berúbm. te Mabler geconterferyet / Dafer penno Die Éopien benn Aldrovando, Gesnero une anoern / miemol nicht fo gar eis gentlid/genommen noveden. Dannes berichtet Geotge 2(nderfen unfer Sals Desobigt su Sroppe / wie aud Jobann Sacob Merd'lein / fo in Java majore

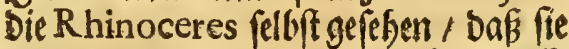
fabmargethau von farben/ Jaben Júffe

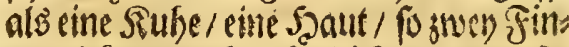
ger Dide / und Eraús gleich als wenn fie gerwapnet máren/ aber nicft von fo bor ben abjegenen / als es in gemein abgebils Det roirb. Senno/wenn fie erwadbent megen ibrer GraufamEeit uno Stártefe lebenoig nicht nool a ufangen. SMercl.

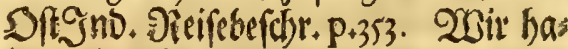
benneben einem StúdFe yon Der Difern

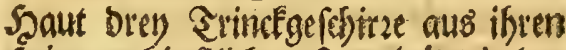
Shornern Eunftlidf gubgearbeitet in Des Sunfisammer fteryen. Sollen auty nider Bifft fenn.

Num. \%. Diefe Szorner ferno von einem Bezoar'Bocfe/ in welchem bes BezoarStein gefunben wird. Das Fhier foll (nach) Aldrovandi2befcheres 23 iii 
bung 1 r. quadr, p. 455.) in (Siroffe uno

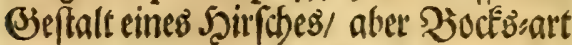

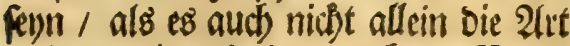
Sormer geben/ fonbern auch Der Name bon Perfifonen 2 Burte is Bez, fo ein Soct beiffet/anjeiget/weil fellige 3 ócfe in Jerfien am Drientalifiben Sbeile fonderlich in Der \&anof fchafft $(E)$ borafan beuffig gefunden werben. Ob aber ber 3lame vom بazar, fo forum, ein Marcft beiffet / ber Eonme / und po wiel hciffe als lap is forenfis, wie Garcias meunet/ la fo muften andere Sachen/io auff Dem Bafar verEauffit merben / auds ifren Damen Daher baben.

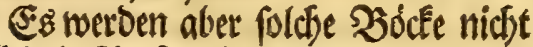
allein in Jyerfiem/pondern auch in Sinois en/Da er Bezar genant wiro/gefunden. Die Đerifictsen mill man bodf für Die beften falten. Es werben aucb Bezoar Steine in 23 eft: Jndien/ fonberlich

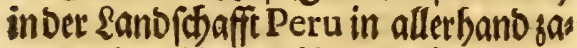
men uno wilden Sfieren gefunden/ fo Etein uno gros / etliche wie Taubens Ever/wie wir bann auds einen folchen/ neben einem Drientalif bsen/folang uno fómal/als einen Finger/ haben. Jim 4. Sucbe Der 28 eft 9 Inb. Sciftorien cap. 42. meroen felbige Steine beforites ben. Es foulen gifftige Sráuter fern/ welche biefe Thiere Eauen uno freffen/ Dabon foll Der BezoarStein machfen. Die Orientalifden follen Dodh an sus

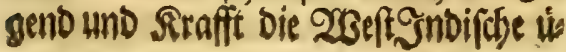

bertieffen. Das audy wilde Seseifen reuno/Die Siiffe ofme Stjaden weiden fónnen/bejeuget Plin. l.10. C.27. Der Steinnirb in ber Sfieren Magen ges funoen/fat im Centro ein Forn doer

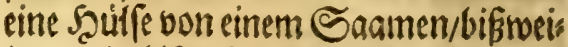
lentumb/bisweilen lánglicht/úber wel/ đees ber Bezoar wách pet/ uno gleich alb biel Schalen úberemanber fid leget/ wie ich folches in şankurg bey meinem guten Freunbe Seel. Szerzm Jaul \&ans germann / Der folche, Driental. Steine viel Gatte/ und oerer etliche / umb mich Deffen ju verftánbigen / enģwer) foblug/ felbit gefehen. Selbiget Stein wiro in 引erfien foch gehaltem/uno wider alle gifftige uno anfifectende Srancefleiten gebraucfer. Die von Denen fo auff Dem Ejebirge feyndie beften.

Num. 8. Iftein Sourn vom milben Odsfen / Der an Bieftalt als ein Şirifo fich befindeet/ hat bas Sorn mitten aufm Siopffe/mách pet eine şand hod erft als ein cinf̧eln Şorn / und ergeuff fid bers nach in etliche Enoen. Soll /wie Julius Cxfar in Commentario lib.6. fchreibet/am Şarkge gefunden werben. Eft bos, inquit, cervi figura cujus à media fronte inter aures unum cornu exiftit excelfius magis directum his, quæ nobis nota funt cornibus, ab ejus fummo ficut palmæ ramique late diffunduntur. Disift/ oefien o: ben pag.II. gebacbt morben.

Num. 9. Sftein Szorn yon einem 


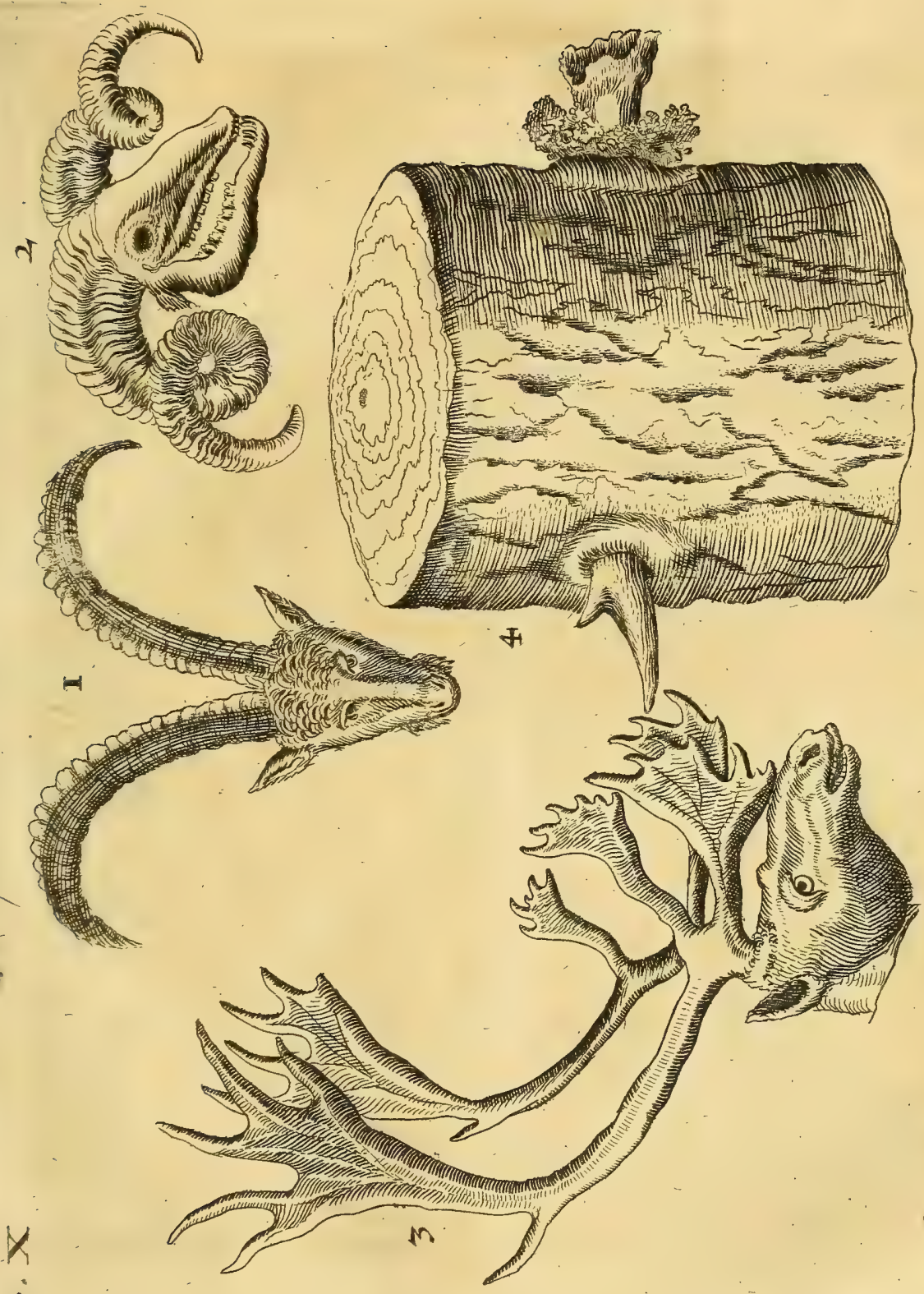



Onagro voer wilben Sndianifchen 2BalbEfel / Das einig mitten an Der Stirn frehet / uno Daber nol tonte ein Einforn genant werben / wie e geoacht / von Den 7o. Interpretibus bat wollen für Das Reem angeferten swetben. Es foll eines von ben grats famftenuno fdyablidyften Shieren peyn/ uno wiro befdrtefen bom Aliano, Ariftotele, Philoftrato, A pollonio, Plinio uno Solino. Uno baben es viels leicht alle aus bem Ctefia. Sb aber fol

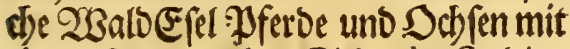
einem Shotn an ber Stim in Smbien fernn mugen/zmeiffelt man billidy / weil toeber Sino (chot noch andere / fo etliche Jahr in Der Saguptitadt Goa und an anoern Drten in Inoien fich auffigebals ten/ uno nodj táglits von Den Europeetn burchgereifet unb gehanbelt rotro/ Feinerbergleichen Sthiere / fo nur ein Shorn vor ber Stim/gefehen. WBas stwar Vartomannus von Den Einfot: nern zu Mecca betieffet / mill Bochartus auch in Brweiffel giefsen / uno nenn

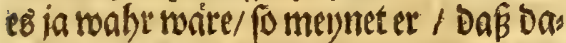
felfit Der Oryx befbriethen merbe. 2 . ber wie fan baffertbe Fhier Oryx und Dafer Reem getwe fen fenn / meil Bochart. mill / Daß Das R eem jwely Scơt: ner gefiabt. Lund will zwar betweifer nichenur aus Den C Galbeetn uno alten Sceltreern / fondern auch aus Deur 2 tras bern / Daf Reem ( fo Die Iraber (E) $\operatorname{Rim}$ nemen) eine 2 (ntberwilden
Biegen/aber viel gróffer uno ftärcéer als Die gemeinen Biegen ferns pag. 962. uno refutiret/ was ihm Dargegen tónte eingeworffen werben. (S)leichnol far get er hernad) pag. 966 : Proinde ficuti nobis deerunt certæ probationes, liceat agere per conjecturas, donec plus lucis affulgeat.

\section{TABULA X.}

Num. I. Seund Şórner von einem WBilben oder Steintode Ibex genanot/ fo fich in Dem Sdjwetisere-(jebirge bes findet. Diefe Scoúrner feyno anderts balt Ëllen lang. Bellonius fdyreitert/ Daßer erines von vier Efllen gefehen fa: be. Es foll unter allen Shieren feines fenn/Daś länger Scúrnet habe als Diefes. Sie follen gefóninbe laufen / und ges maltig/ia voneinem Felf́f sumt andern/ wenn fie gleids 8. Schritte von einander fetehen/ fpringen Eónnet/miejonft onius Qus Aldrovando pag. 80. berichtet.

Num. 2. Jit ein Siopf oon einem Americanifchem Bocfe / fo von Dero Sorten in Scollano gebracts trovorden/und ift su Encthufen geftorberm.

Num, 3. Sftein Ropfi und Getroir

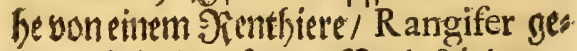
nant / fo in ben fernen Nord: \&ándern/. fonberlich in Eapland falten / feften Derr Shirfchen nicht ungleich/ baben Doch gar

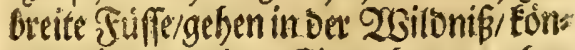
nen aber bon Den Einnoghnern sabm 
gemadiet und gur şaubbaltung ges braudit twetoen. Etlitbe Eappen follen Detfelben 100, 200, 3000, uno tool melbr in ifren Şofen baben/oie fie ourd eimen Scirten jut 2 Seide aus sund cim treiben laffen. Sie retoen wie bie Sube gemolden/fo gute Sutter uno Refe geben forl / meld be ifnen niche nur zur Epeife/ fonberm aud fur 2irteney wiber gewiffe Mángel uno Brebrecsen Dienet. Shre befte Speife ift \$noos I foan Den Sáumen uno auff şugeln waidy jet. Sie werben aud) zur arbeit gervehnet/Dás fie nicht allein Sd)litten/ Sendern auch Eaftwagen siehen/mit wels

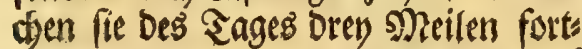
Eommen Eonnen. Mit Sdblitten auf Dem Schnee Eonnen fie úber alle Ma af: fe gefdrwinde lauffen/ uber Berg uno shal: uno ferno fonoerlich bie / fo su Dem IJoft \&auffen gewebnet / Des WSes gez fo Eundig / Dap fie ofn einigez \&en cfen uno 2 Sincfen fich an beftimtenSit einfinven / Dafie alsoann autf ftille ftes ben / uno ficd nidjt ferner jagen laffery weldhes alfo nicht / wie etliche mennen/ mit Sexeren sugebet. 23ienol bie Sexerey bey Den Sappen nicht gar un gemein/Dáp menn fie zur See feyno/mit 2ufflófen etlicher Snotten / Den Wino zuifrem Willen baben fonnen / wie man von ifsuen fagen will. Selbige Sienthiere / wenn fie in andere \&ander uno \&ufft gebrad)t werben/ Eónnen nicjt lange leben. Waie es benn offt verfudjet norben/ uno beridjtet Olaus Magnus, Dak einft Dem Saerizog Frieorich juşol fein aus Sdbreben 6. Derfelbigen mit einem Snedte zugeforict worben feyno aber fampt Dem sinechte bald gee ftorben.

Num. 4. Sit ein Siefe: (Siencibe Durch einen $\mathfrak{Z}$ aum gewoaijen / nach Art Det Rinbacte / fo in Det Rónigl. Sun|t Rammer zu Soppentagen bes finolich/und oon Olao 2 sorm befortie ben mird.

\section{TABULA XI.}

Num, I+ Ifteine gat foroine Sdian ge/ uno niemano fídádich/ wirb Zicatlinan ober mater formicarum, Die \$utter Der Ameiffen genant/ weil fie in Den sódberm und Sauffen/Da bie?tmeif fen niftew/fich auffibalt / unb aud) ibnen folget/ ift Eaum eines Finger's Dicfe uns anderthalb Ellen lang/wiro beforrieben vom Nirenbergio lib. 12. c.7.

Num. 2. Diefe Sdlange wiro ges nant von den 2Imericanern/ wo felloft fie am meiften angetroffen wiro Tenthla. cacauhqui, oder Domina ferpentum. Die principalfte unter Den Sd langert. Sift Die allervergifftigfte uno fódiolids. the/ Darumb bat fie Die Natur geseidonet/ Das man fich vor fie futen fold. Dann fie finten am Schwantze etliche Sslies $\mathrm{Der} /$ fo fich berwegen uno Elappern / bat. 2 Senn man bon ibr gebiffen wirb / uno nidgt 


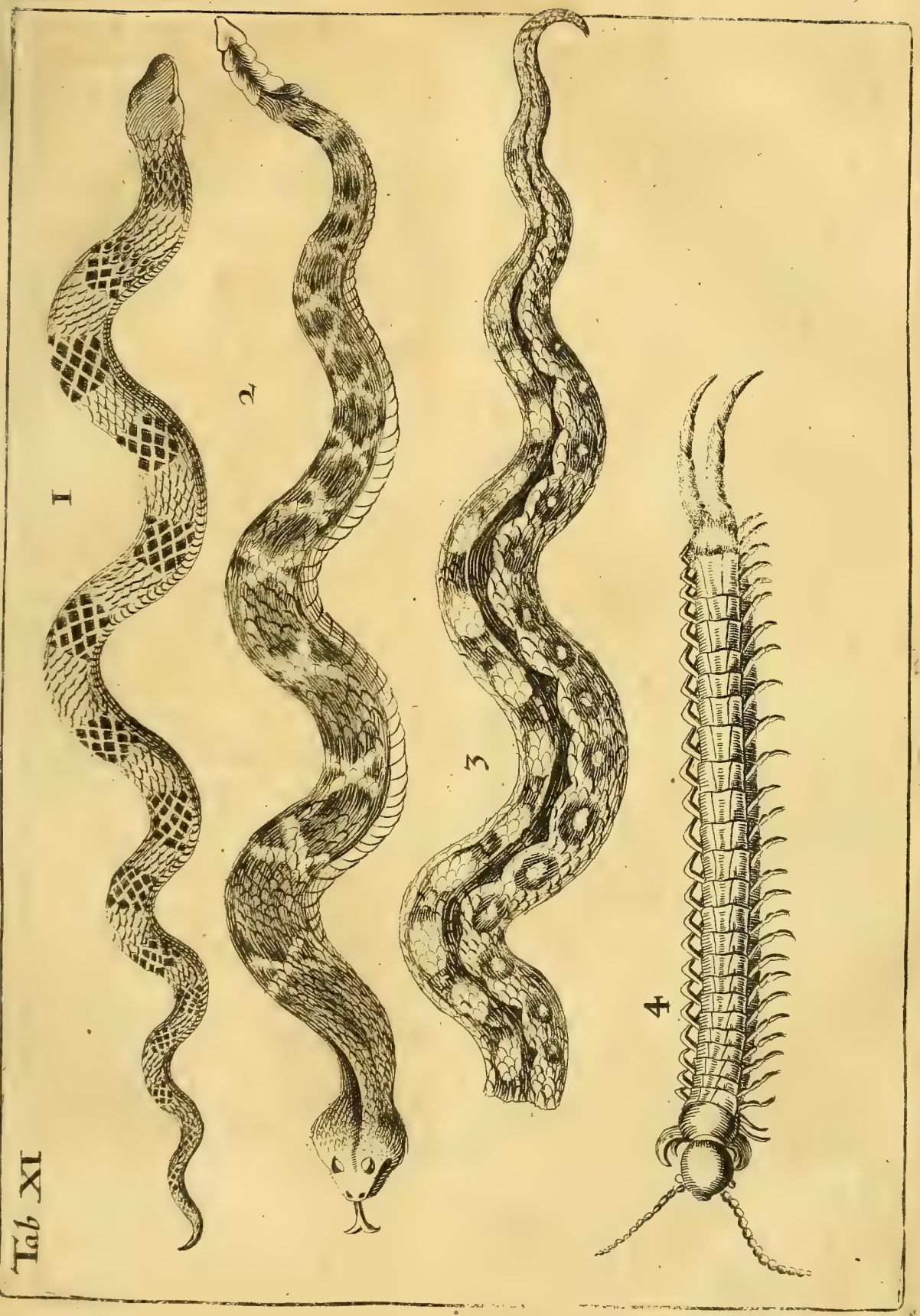



nid)t alsbalb etwas bargegen geltratt: cher / mus ber Mench innethall 24. Stunbendes Todes fenn. Das befte remedium bargegen foll fern / wenn man nur alsbalo Das verlerste (S)lied in frifche Eroe foharzet / und fo lange Darin hált / bi ber Sdbmers vergehet.

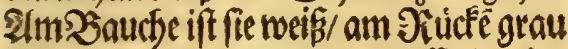
mit weiffen Ereukentoer Siopff als eine Wiper. Die/ fo bey uns a finben/ift น́. anderthalb zoll bicke. In Mexico follen fie als ein Sein bide gefunben werben. Die Mexicaner niffen fie sabm su machen / Daf fie felbige in ibren Şáufern jur \&uft baben/Eanein gank Sabr ohne Speis uno Iranck lebeny Darbon STirenberg Cap.I.

Num.3. Sit eine ausgeftopfite Saut von einer gar groffen bunten Scjlans ge / worbey auch eine von bergleidsen/ Eommen aus Sirafilien. Soldte 2Art Schlangen follen ein Schweinfercten ver (chlucten fónnen/welchez mir zwar anfänglich) ungláublich) vorgetEommen. 2rseil aber etlicse/ fo in Jnoien Dergle: chen gefehen/ und in unfer Sunftsams mer bey?dnfortaung ber unferigen felbft anfiengen zu erzeflen / wie in Der Snfel Formofa von felligen Thieren an Scus nern und anderm fleinen Viefhe / fo fie verfchlucfeten / groffer Scjade gefoses he/habe ichs gegliubet/ und if mir nach Der'Beit befto glaublicher vorgetormmen/

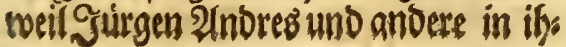

rer Sndianifchen ?ieifereforeibung bes richten / Dak auff Der Infel Java Schlangen gefunden merden/oie gante Mienfonen verfchlucten. Arift,lib, an imal. c.I5. er seflet / Dak etliche Africanifite Meer: Scblangen gefunden wer:

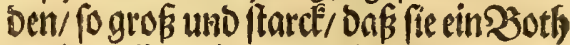
untrwerfifen Énnen. Uno im Olaa Magno liefet man/ (lib. 2I, cap, 43. tweldbes nod) mefre zu vertwundern) Dafis in ber Plortwegifhen See fich Sthlans gen fehen laffen / bie 100, in 200, uno mefre fus lang / uno von getwaltiget

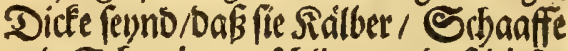
uno Schweine ver fablingen/ Daf biefes wafre fey / bat neulich ein vornefmer

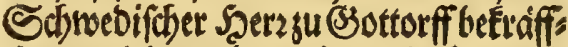
tiget/ und barneben gefaget / Daper vom Súrgermeifter zu Malmoi einem glaubruirbigem Manne gehóret / wie

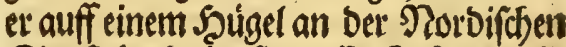
See tethend eine fo groffe Edslange in ftillem 23 affer gefehen/ bie von ferne po

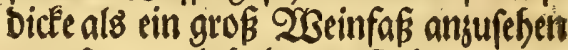
getwefen / und habe 25. Frummen ges babt. Selbige Sdblangen follen nue bey ftillem 23 etter uno ju getwifien Beis ten fich auff bem 23 affer Pehen laffen/ uno wenn es gef rbiebet / fofl eze ein lós Omen Doer 2Inseigung fúr Das \&ano o: Der Şerzan Des \&anbes fenn. Olaus Magnus fez̧eret zum Exempel. Daf Anno Is 2 t. eine foldte Sdblange fich bod uiber Das $23 a$ ffer mit einem Stheil

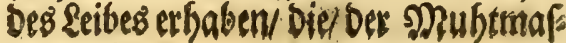


fung nad/bey so. Ellen lang getweren/ bie foll Des Chriftierni, Tyranni Sós nigs in Dennematef unb Sobmeben o: ber Chriftianifecundi unb vieler bot: nefmen Saeran bes Lanoes Untergang angebeutet baben.

23as Poffidonius, citanteStrabone lib.16. von einer fo groffen Sकlan ge bie im felbe Maera todt gefeben worben/ Daf toenn Sieuter auff benden Enben sebalten eimanber nid)t baben feben fónnen/beffen Biadje fo grof ges wefen/ Daf́ ein Seuter mit Dem Y ferbe binein reiten fónnen / uno sine Schups pe son Deffen Saut grófer als ein Scbilo. (Solche 2lut Şblangen bat man Draden genant.) $2 B a b$ aud Olaus Magnus aub Dem Volaterano erzeflet/ Dấ Scblangen gefunben tour: Den/ weldse 1000. Sditte lang / unb zu getviffen Beiten Des Sabr cine ganţe Seerbe Elein Diebe fampt Dem Sirten auffireffen Eomnen/lapi id an feinen Drt geftellet fenn.

Num. 4. Sftein Scolopendra, ein Crbober 2 saffer: $2014 m$ fonft megen ber vielen frufte / Saufenofü genant/ Aldrovandus de infectis lib. 7. c. 6. mach)et berer ztweberlen (siefoblechte/etlis be fo im 20 affer / etticbe / fo auff Dem sande leben.

\section{TABULA XII.}

Num, 3.2. Seyno Scorpionen/Des ren (Siroffe red) geconter feitet / nad feis ner Siroffe uno S3eftalt. 2Siro nidst leid)t to grofis gefunben / ift fdyroars. Yair baben biefen aus Jerfien von bet Staot Safdoan / wopelbit fie bauffig uno am allerbergifftigften fesno / mits bract5t. Der Stadjel / Den er Frum am eufferften Siliede Des Sdjwankses bat / mennet Plinius lib.II. c. 37. (21) bobl/Daburd er feinen (Sifft / gleich aud bie Soblangen burd ibre boble 3ábne in bie 23 unbe gieffen. 2lbet es ift in Der anatomia befunben toorben/ Daf Die Sdblangen unter ben Babnen cin Sláplein mit S3ifft baben / uno in Seiffen auforucfen. Sobeinet gleids? wol/ Dak bie Spige / pals eine fubtile Tatel ift/ Fein Sod babe / fonbern gans fev. Sie flieben bie Sonne/ uno bals ten fich gemeiniglich in ben Siken bes alten Maurew/audo unter ben Sttinen auff / fie werben nicht per coitum generieet/fondern aus faul \$̧olge und ans Derer faulen 2la sibaffen materia butch Der Sonnen Saige / fo Dem Eliano zu glauben. Die Jerfer mennen / Die Sungen folten bie Alten tobt beiffen. $25 a s$ biervon uno von ibrer generation mełr zu wiffen/ fan man ausfübes licher lefen in meinem Berfifhen side fenthal lib. 7. Hift. 10. Sie Eriecten gefdsinino auff Der Eroen / uno balten Den Stjwankempor / fonft muirbeek ifnen wegen des Erummen Stadjels verbinberlic fallem. Diefer nutrbe in 


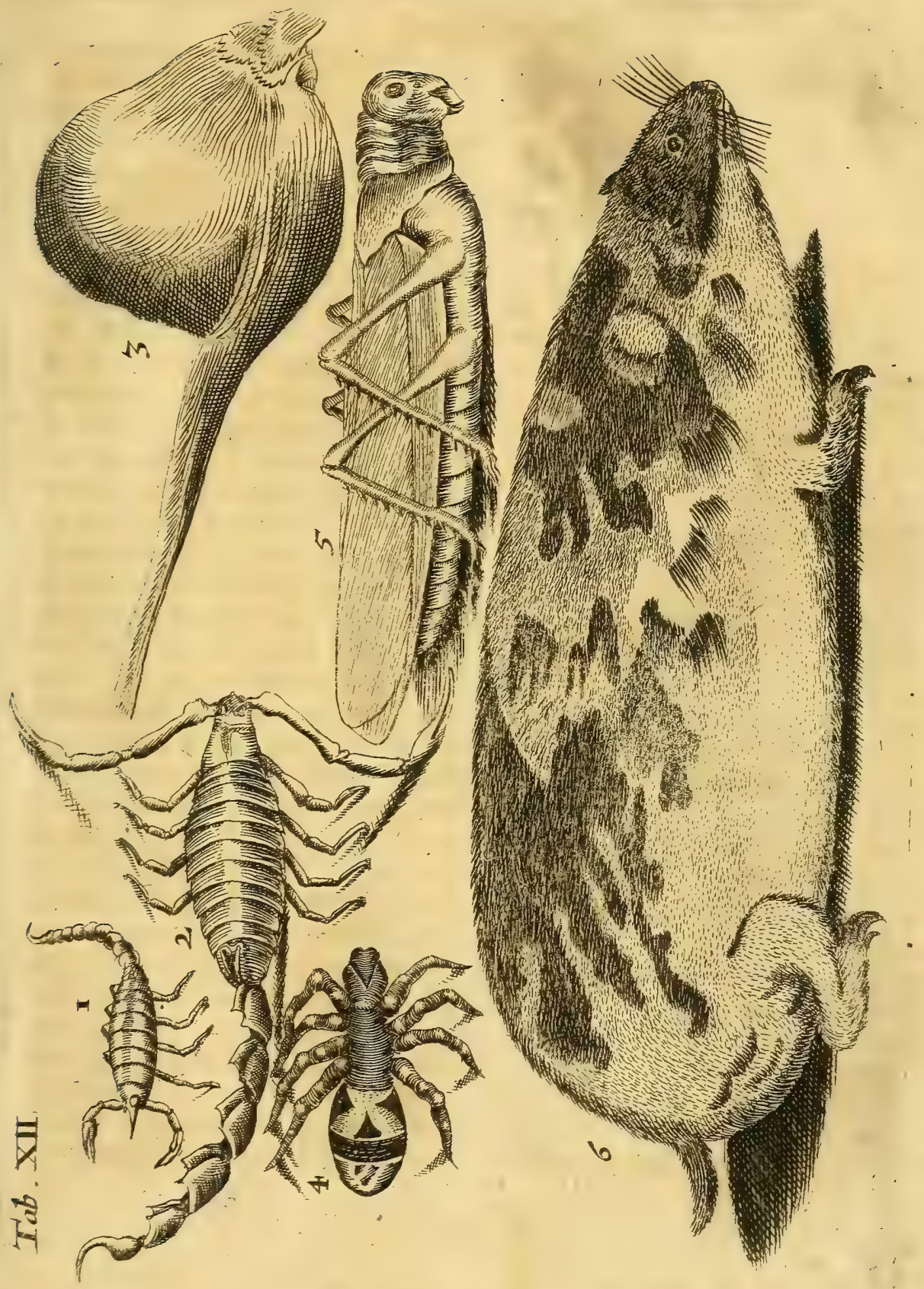





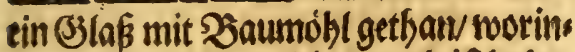
nen er úber 8. Tage lebete / unb ift baber su glduben/was Aldrovandus aus bem Alberto lib. 5. de infeet. pag. 581. hes ridtet / Daß fein Scorpion 22, Tage im Dele gelebet/ Da andere Infecta formt im Del bald fterben. Sie Eónnen fonft obme Speife lange leben / gleidy twie Die 2irt Scblangen. Und meynetPlinius

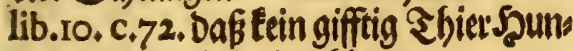
ger uno Durft feerben Ésmne.

Num.9. Ift ein munberlicher Stnos d)en oder Bein/ faft wie eine jufammen getrochene s)raus ansufeben / eftras Eleiner als ein STünerEy. Sat einen bewes lichen Schnabel/und einem als eis ne Sederipule bicken Sdimants. Olaus 230 orm batraud) Dergleiden in feinem Mufæo gebabt / fo ief̧o in Shbret Sớs nigl. Najeft. zu Dennemarct אunfts Rammer verbanden. Es haben zmar eflidse gemennet / baf es foll aus bem Sopffe eines Jif fenn/wie audf bergleidsen in Der S d)aks Cammer Des Eresherḱgog Ferdinandi Caroli auffoem Shlok Amris anstu treffen/mit ber Uber[drifft: Os R emorx. 2 ber es wirb yom Olao $280 \mathrm{rm}$ gat rech)t refutiret/ bann Des Remoræe Sopff/ Derer wir gnoen baben/ift biel gu Elein/ auds nicht barnad) proportionitet baper ein folch SBein faffen Eónter bleibt alfo cin unbetandt Bein. Ez fen Dann/ Daß man barbor halken will / Daß es in ber Eroen generiret fer / wie bann
Die Naturkundiger fohreiben/bẩ untets

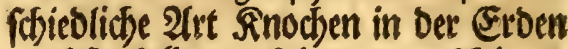
wadden follen. Şiervon gar fónón fu lefen Athan. Kircherus in mundo fubterraneo lib. 8. fect. 2, de Enoftrorum, five osfium fubterreftrium ortu, melder Deffen Beugnif fe aus unterfobieolichen Autor, hets ben bringet / Die Darbon geferries ben / als Hector Boetius lib. de lapidib. Agricola lib. 2. defosfi lib. Ercker von Bergmercfen. Cxfalpinus cap. 84. hic dicit : Offa autem è terra nafci invenirique lapides offeos luculenter oftendit Theophraftus \& .

Num. 4. Tarantula ift eine $\mathfrak{A r t}$ von grofien Spinnew/Fo von Der Farens tinif chen Biegeno ober vont Fluß Thara Den Namen betummen/ weil fie Dafelbfe am meiften/ wieaud) iescho in Calabria unb Sicilien gefunben werben. Sie baben einen nounderlichen (Biffit bey fich/

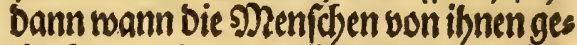
ftodsen werben / lauffen etlidbe / etliche lachen immer / éliche meinen / etlidse

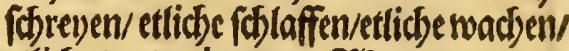
eftiche tanken immer. $23 a 8$ vor remedia Dafur / fonberlich Den Tänsern/ burch eine fonberliche Muficalifobe harmonie und Melobey / if benm Kirchero libro de arte magnetica parte 3.t.8. c.2. ausfúbrlich beforries ben. 2rsas bie Jerter fúr ferlgame Euren bey ibren Siefobabigten vorneths E ii

men/ 
suen/ift in meiner গJerfianifocen Sieifes heidfreilung / bey Ertwehnung Der Stabt Kafchan, wofelbft fie baiufig ansutreffen/gebacbt morben.

Num. 5. Sfte eine Sacufdrecte / wels the Doctor Paludanus fellbft mit aus 2(egypten gebradt.

Num, 6. Sit ein fonderlich rauch Shierlein / etwo ein wenig groffer als eine 刃hauß Fommt aus ?20rwegen/wo: felbite es aus Den 230 olfen gefallen. Diefe 2at follen auff Dem felbe Die Saat uno 2 Seibe fehr verderben. Sin Nortwegen serben fie Leming und Semmiger genant / beiffet fo viel / als Meffores, Ednnitter / weil fie mit ben forberften Baibnen gleid) als mit einer

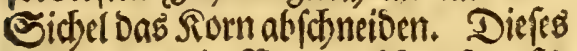
Shierlein (Die Mortwegirthe Maús) hat Olaus 230 rm in einem abfonderlis chem Tractate, wie auch in feinem Mufro auffübrlich befdrieben. Damit nidts baran ju gmeiffelm/ bap fic aus Den 280 ofeen fallen fuibret er gar viel glaubs sourbige Exempel mit ein. Mran hat befunoen/ Dap fienicht allein auff́şand/ fonbern auch zu Den Siffoern in Die Siabs ne und 2000 the gefallen find. An 165 I. foll (referente Wormio) im $\mathfrak{C}$ afpel Sogno einer Erancfen Trauten / fo uns term bloffen Scimmel gefeffen/eine polche Mauf in Sç⿰亻⿱丶⿻⿰丨乛巾 gefallen fenn. Man fou fie autch sur 2Binters: Deit wiber ben Edjnee / auffhohen Bergen gefallen/ finden. Olaus 20 orm erseblet auch!
Daf stweene 2aaren in Norwegen úber cinen gefrornen Strom gangen/und als fie mitten auff Den Strom gefommen/ fer) Demenen eine Srote auff Den Sut gefallen. p. 32\%. Diefer Máufe fél aber if bund mit fobmars und rotben Flecten/gleidj mie bie Shamfter / Deter umb NagDeburg und 21/chertelen / in meinem 2 aterlande und umbliegenden Begenden biel gefunden werben / und am reiffen Siorn groffen @chaben thun/ Dann fie bie befien Sórner in Die Zellem/ fo fie unter Der Erenen baten / bauffig einfchleppen. Und halte ich Darvor/

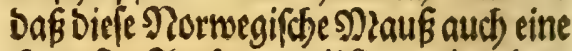
Samfter:Atrt fen / weil fie / wie anbere Şamfter / menn fie angerúbret werben/ bo Bbaffi auff Die Schinterbeine treten/ und fich zut Bjegenwehr anfellen. Dies fe Norwegif che Mzálufe follen nicht nur Den Felofruiteten I fondern aud) Den Menfdenfchiolid feyn/und fonberlich nofiefich hauffig finden/ bie \&ufft vers giffen/Dás Daber Die Jefte unb anbere sefábrliche Srancefbeiten entfanben. Don biefer Nortwegifónen Nauk bas ben auch gefchrieben Olaus Magnus hiftor. fept. lib.18 c. 20. und allo ihm Gesnerus cap. 17. Art. 2.Scal exerc. 192. fect.3. Fort. Licetus 1, 2, c. 46. de his, qui diu vivunt fine alimento, Zigler in defcriptione Norvvegix. Unter allen aber Eeiner auffuibrlicher als Der geleffrte Olaus $2030 r m /$ roeldjer bon ifrem Ur uprung nicht ungláubliche 


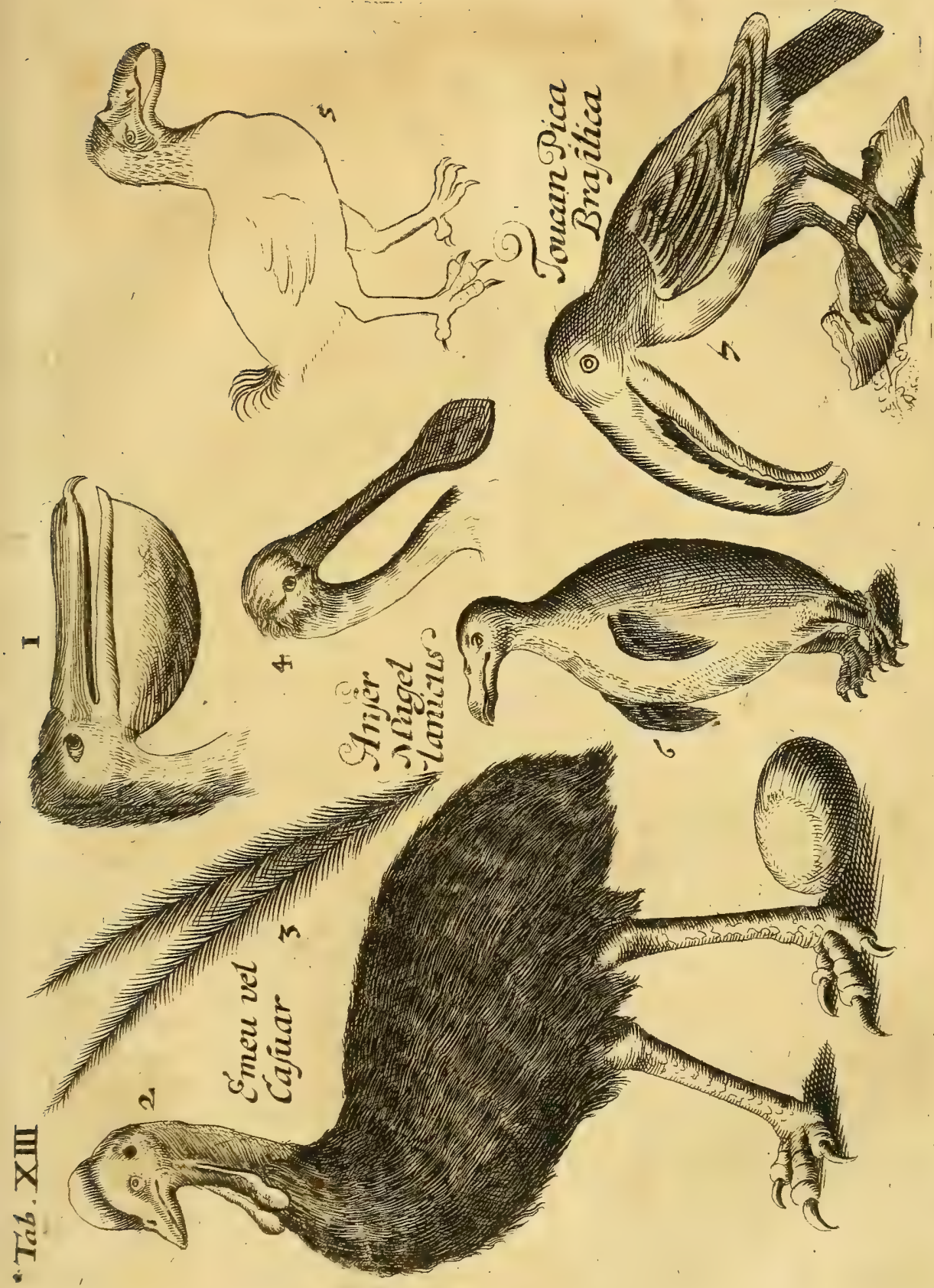



Opiniones fat 1 tmo fonft fine notable Shiftorien mit einfüfret. 200 ors bin ids Den gưnfigen \&efer will angewies fen baben.

\section{TABULA XIII.}

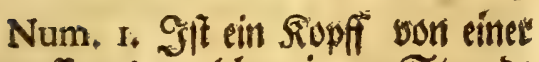
Sropffigang / weldye wir am Strande Der Eafpifcten See gefoboffen / Da fie bey so. in roo. beyfammen ansutreffen. Diefer Boger if etwas gréffer als ein

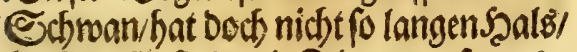
fonft an Sieftalt und Fetern unfern ges meinen ङianfen gleits. Zion Den Siuf fen werben fie Babba, bon Den Yerfern Cuthan, bom Plinio uns Aldrovando Onocratalus item Pelican genant/ hat forn am Sdjnabel einen flummen Saten/ uno unter Dem Schnabel und Sefle einen \$Beutel vori Dúnner Jufam: men gefocrumpener Saaut / welldbet fich fo weit auboebnen láffet / Dás man mit einem Stieffel ober אopff geraum bincin fabren Ean/ ims felben famlen fie

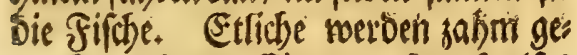
madit / und am Eingange Des Salfés

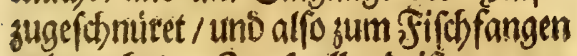
gebraudset. In saollano if vor wes nig Sahtern eine foldhe lebendige (Jans

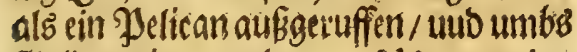
Oeslogeféget worden. Aldrovandus

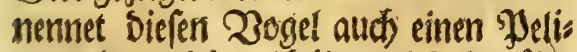
can/aber gleidjwool hält er nid)t Darfúcy

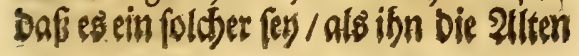

gegláutet/ und yon Den smablern abges

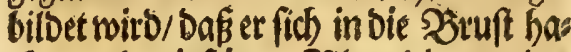
ceen/ unto mit feinem 23lute Die von Dee Sdjlangen getobtete Sungen wieber lebendig machen foll / welches swir mit Dem Aldrovando fur eine Fabel hals ten. STieson if aud in Olearii Đerfis

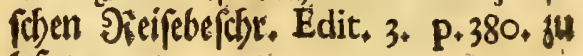
léen.

Num.2. Iff auch ein groffer Snbias nifiber 2 ogell weldben bie Luferigen Cafuar, feine Eanosleute abet Emeu nennen. Sie follen am meifen nuff Der 'anful @umatra gefunben werben.

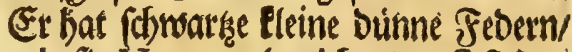
wie fie Num. 3. besecichnet. SJft Ders

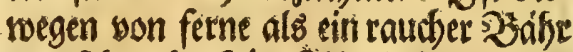

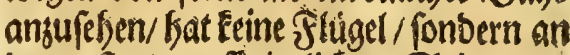
Dero ftatt auff ieglicher Seite gnoo

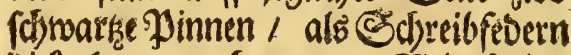
Dicle berunter Fanigen. 2 Sir baben Diefen auff Gottorff lebendig gehabt. Er war now einft fo grós als ein Sdjwän. 23 senn er fidty auffiridtetel war er faft Mannes \&ánge. Er batte

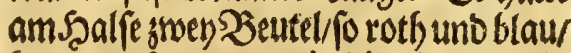
berunter bangew/ noie bie Ealcumifote Scatinen. 223ar fehr mubtig uno Ecce' gieng auff einen Snaben ober Eurkge

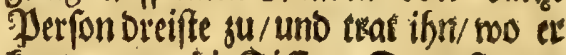

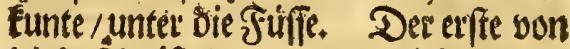
Diefer 2̂trif if Anno 1575. mit Dererfen J̧oulandifonen Sdjiffabrt aus Sndien von ver Snfer Banda, wos fehr viel Mu: featens? duffe madyen/ mit beraus ges (ciii,

brachts 


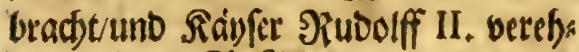
ret noroen. Cluf,lib.Exotic.5 cap. 3 .

Num. 4. Ift ein Siopff bon Det Reffelganf/Derer wir autb viel am Ca: fpifchen Strande angetroffen / wirb Platina aud Cochlearia genant. Sit ein wenig eleiner als̈ eine gemeine Sanf. In Engellano bat man fie willo und gabm/und werden als delicate Speifen gebalten.

Num. 5. Sft ein Sopfi bon eirtem fiemboen Oogel welden Clufius Gallum peregrinum, Nirenbergius Cygnum cuculatum, bie Sallánber aber 20 alghoogel / bom Ecfel / Den fie megen bes barten fleifocs madsen fols len/nennen. Die Saollánber follen su erit folden 2 ogel auff Der Infel Mauritius angetroffen baben/ fol aud Eeine Flugel / fonbern an beffen Stat stwo Эinnen balen/gleich wie bie Emeu uno Pinguinen. Cluf.exot.

Num. 6. 2Birb Anfer Magella. nicus genant/mir baben berer stwo /Die eine ift bom Freto Magellanico bers fommen. Die andere aber aub Nors Den/fo vor stoen Safien bey uns leben: big getvefen. Ift eine red)te (Ssan [e?2Irt an Ssrófe/fredern uno fúffe/geben auf: gericbt/wie bie snenidsen / Den Echnas bel empor Galtende/baben feine flúgel/ fonbern nur fleine Fittiçen fónnen ber: wegen fich nicht bom sanbe erbeben. Drachen tieffe sócher in bie (Eroe gu ib: ver 230 bnung/ man Ean fie mit Şant
Den greiffen/uno mit Wrugeln toot fकlas gen I wie aud bie Sollánber gethati. Sie baben feltige 23ogel ben ber Cabo de bonaEfperanza baiuffig angetzoffert. Sollen bart von Shaut I weldse etlicbe Der Einmobner gu Sileibern getraudben Dab Fleifo aber gut zu effen feun. Sie ermebren fich oon fifichen / Daber fie fid meift auff ben Eilánbern Doer Infelin auffhalten. Clufiuslib. s. Exot. cap. 5. wie auch Olaus $230 \mathrm{rm}$ pag. 300. befdyreiben fie ausfubetidjer.

Num. 7. Sitein Stbmabel voneis nem Vogel / von Nirenbergio $\mathrm{XO}$ chitena Cati, bey Den Prafilianerw ba ex gefunben wirb / Tucan genant. Sit fo grofis als ein Spect)t/bat einen Sdjnabeloer fo lang als Der \&eib itt nemlich 8. 30 lluno 3. 3oll breit. Mnan folte fich vertwundern / roie bas ein fo Eleiner Jogel einen fo groffen Styna bel tragen fonte. Ift aber gat bohl uns gants leicht/mic ein Stjwam. SMan fol ben 2 ogel Eónnen sabm macjen/Daper im Scauje niftet uno brutet. Marcgravius hiftor. avium lib. 5. cap 15.

Aldrovandus fdyetibet aus bem Theveto, Dafi bie jenigen / fo in Bjrafe lien rebfen/ berichten/ baf biefer 20 gel fich oon J feffertornern nebten foll/wels d)e er geişig einfdylucten uno unbers Dauet wieber bon fict) geben foll. Won foldsem 3 feffer bie Brafilianer mebe als von bem andern/ fo fie bom Stamm nebmen / balten follen / in seynung/. 


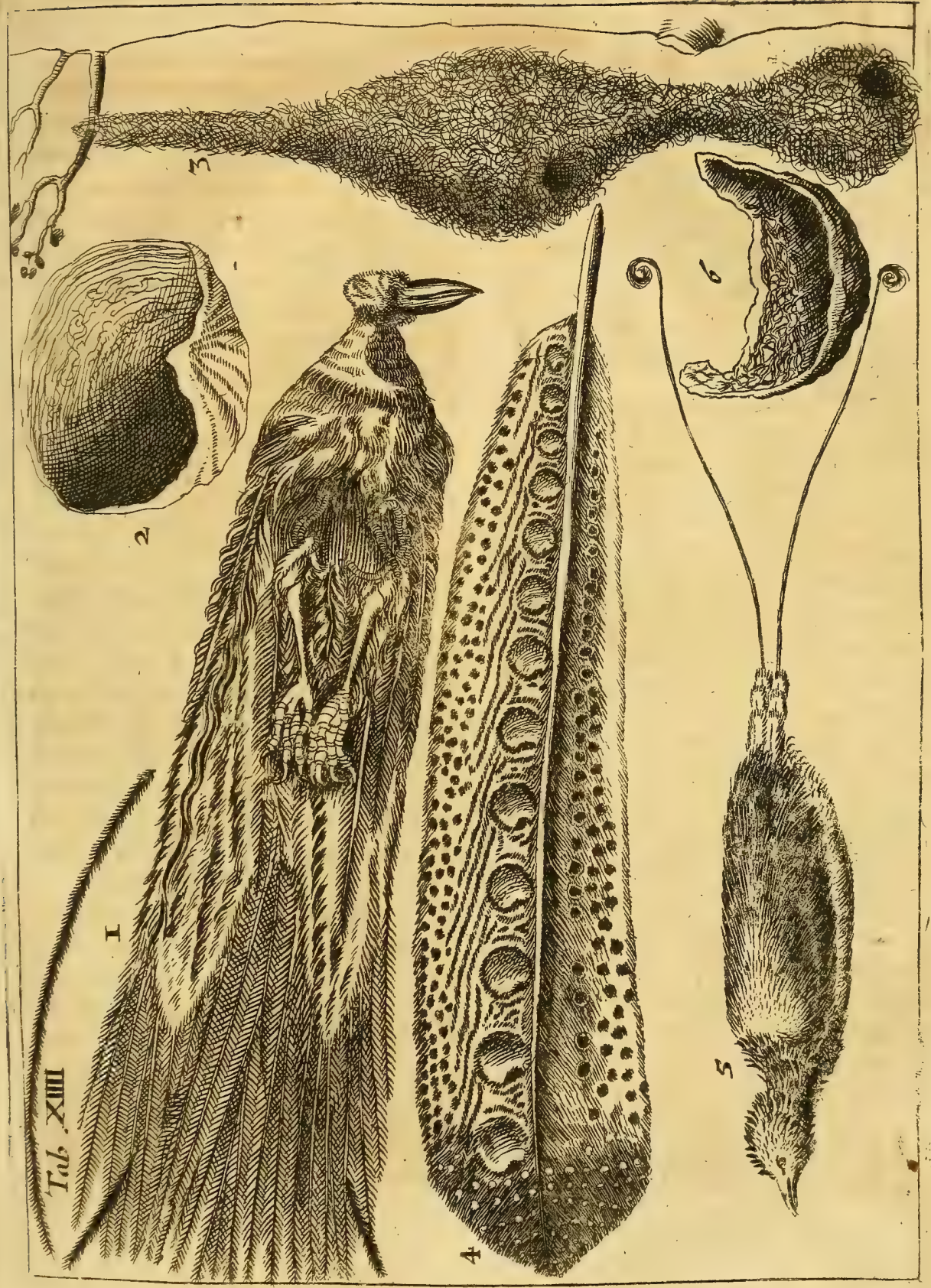





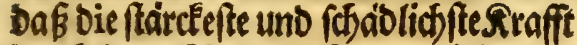

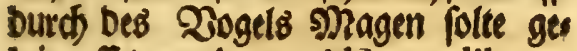
bámpffet werben. Aldrov. lib. 12. cap. 9.

\section{TABULA XIV.}

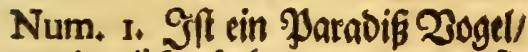
berer wir etliche baben/ man nennet fie alfo/weil etliche Dafur gebaltem/ Das ins Dem man ifren lufreprung nicht weifst fie aus bem Yarabeis/fo noch auff Ers Den fern fod hertommen / auch) weil ihe Sierabt an Febern úber bie gemeine Zogel/ merben fonft auff Den Jnfulen Moluccis Manucodiatæ h. e. (S)otteb Zogel genant. Man bat/mie Aldrov. fobreibet lib. 12, c. 20. ihren 2nE uno no fie niften/ nie recbterfabren fóns nen. Ex ergeflet derfelben fünff Species, von Denen wir breverlyy baben. Dafi er aber faget: Omnibus tamen illud peculiare eft, ut pedibus care. ant, Dak fie alle feine Júfie faben follen/ Derbält fíd nicjt alfo / Dann wir baben Drey/fo fúfe baben. Sch bin berichs tet worben / Dák bie Einwoobner in In Dien ifnen/wermafie todtauff Der Erden gefunden werben/alsbalb Die Fuiffe abs brechen follen / umb felbige unter ifren 2Bahren beflo beffer einjupacfen. 2tber einen Regulum haben wir / Den man Den Siónig ber \$arabi is: 230 gel nen net/ wie num. 5. abgeseichnet / Der ift auff bem Sopffe berunter $\mathfrak{B}$ lutroth/

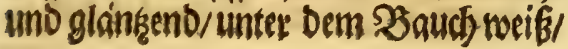

febre anmutbig anjufefenthat Eeine Truf fe gehabt / fondern mit zmenen langen Stralen/ale Pferbescaare / unten mit einem umbgetrumten grunen FeDeris Wen/fich an Die S3áume hangen muffen/ mie Aldrovandus meynet. Sift Eaum einer guten Şandoreit lang. Uuns ger Dencfet Diefer Autor ferner / Dak bie Jef đaffenbeit Diefer Wogel Den Róni: gen Marnin in Den Infulis Moluccis Weranlaffung gegehen/3ugláuhen / ba

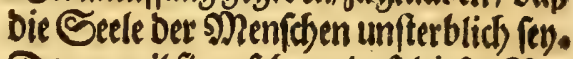
Dann weil fie gefehen / Daß̉ Diefer 20 : gel nirgeno auff Der Eroen geferfen/ auch nicbe fikgen Edonnen / fonocrn nur tobt aufi bie Eeroe gefallen / gefunden reerben/baben Die Mafumedifen / fo mit ifnen Rauffimannichafft gettrieben/

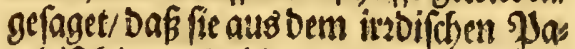
radi fámen/ bahin Die Stelen Der 2 bs geteibeten fübren. Dafelleft toare úber alle maffe groffe Freube von allerhans Suft/ wie Die \$Mabumebiften ibr \$aras Deíp fleifostich zu befotreiben pflegen. Dáa alfo fellige Rónige zum Mabus medifthen silauben fenno gebracht woors Den. Aldrov. d. 1. Und weil fie Giottes Zogel genant werben / fo werben fie bey Den Moluccifdsen Siónigen in grof: rem 2 Berth und beilig gehalten. Und biloen ibnen cin/wenn fie bie Febern won Dieferm2 ogel bey fich tragen / Eónten fie im Streite nicbt berwundet rerben/ menn fie aud im Scharmúgel forn an Der Spike ftumben. ibid.

Num. 
Num, 2, \& 6, Sino Woget:9es feres/ Derer wir untertshieblidse baben/

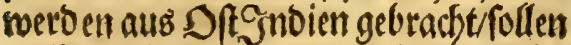
auff Der Cufte Cormondel an Stein: felfen gefunden / bon gar Eleinen 230 s getm/ po barin nitten / gemachet / pernto nicbt gróffer als ein großa ball șüners Ey/yon einer Materia, fo alo Siragant ansufergen/auffeinander gefereset / gleich wie Die 2 rt zu bauen an Den Schmats ben Deftern zu Fehen ift. Eso follen ets liche groffe Serzen feltigeN Nefter in wat: men $23 a$ ferter auffiveidsen / von ben Fes betn / fo man bifmeilen Darts wirchen findet/abjaubern / und in einer Satimets

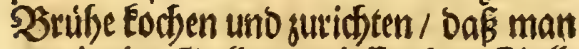
es twie eine BSallert genieffen Ean. Soll/ swie Olaus 230 orm aus Der Relation Johannis de Laet beridxyet / von benet/ qui in caftris veneris ftrenue fe exercere ftudent, gegeffen merben. Vide Mufreum W ormi P.3II.

Num. 3. Seuno jween 2 Jogets fter an eimanber hangeno/won Srafabls mer doer Sheu in einander geflodyten/ follen von Den Eleinen \$Jappagoien / wie fie in Guinea haiuffig ansutreffern/gema doet / uno an einen búmnen fobroancfen Zrveig gef angen werben/Damit fie vor Den Sd) langen/weldye ifnen feflen nadbs: frellen/ficher jenn mugen.

\section{TABULA XV.}

Num,I. Siteine grofle Jndianifage
Flebermaup/Derer wir swo haben/fenno gróffer als bie Fiaben I Der \&eitboum Sopf bi zum Schwants ift eine halbe Ellen lang / bon Dem eufferften gounct

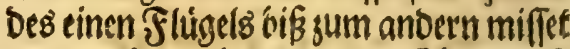
man 7. Duartier oder stwo Ellen wenis ger ein 2 iertheil. Sic follen in Splts und 2 Beft Inoien bauffig fidi finden.

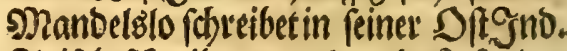
Pieifebefdoreib. pag. 67. Dafi fie Den \&euten in ben Bjarten groffen Sd)abert thun follen / Daf sman offit bes g?adbts 23ache balten uno fie fodeuch) teern mufs. Sie follen ibre Sungen alfo antegen ju faugen / wie in Der Tabel abgebitbet. Plin.lib.10. c. 6r, Unfere aber feyno blok won Jungen. Jonftonius (d)eers

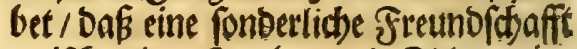
stwifchen Den Sauben uno Flevermaits

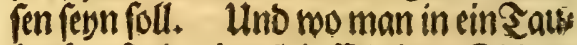
benfaus oben im B̈ipffel einen Fleders maufs Sopffitectet/ follen bie sauben gerne bleiben.

Num. 2. Sift ein Schnabel eints unbefanbten 20 ogetz/melcher auch Dem Aldrovando unbeÉand getwejen. Jonft. p.54 Daber er von ifnen wes Der abgeseidgnet nods befobrieben note Den.

Num. 3. Sfit tin aufigeffopffet Soun aus G vinea, berer wir 6. ettidbe Jaht im Yogelhaufe lebentig gehabe / fie has ben zwar auds Eyer geleget/ aber nicht

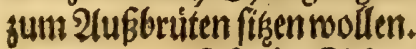

Num. 4. Ift ein Edjnatel bon eis 


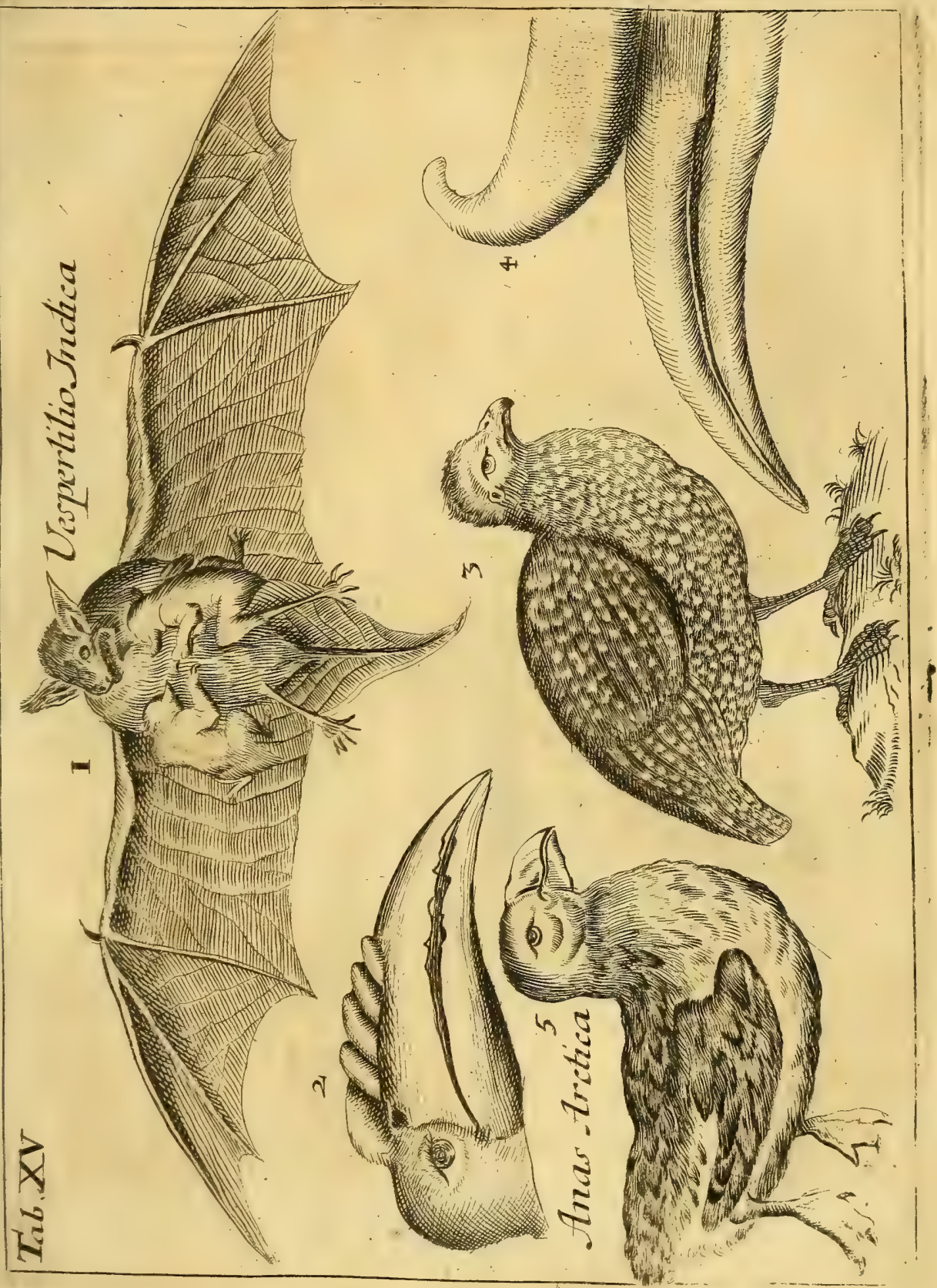





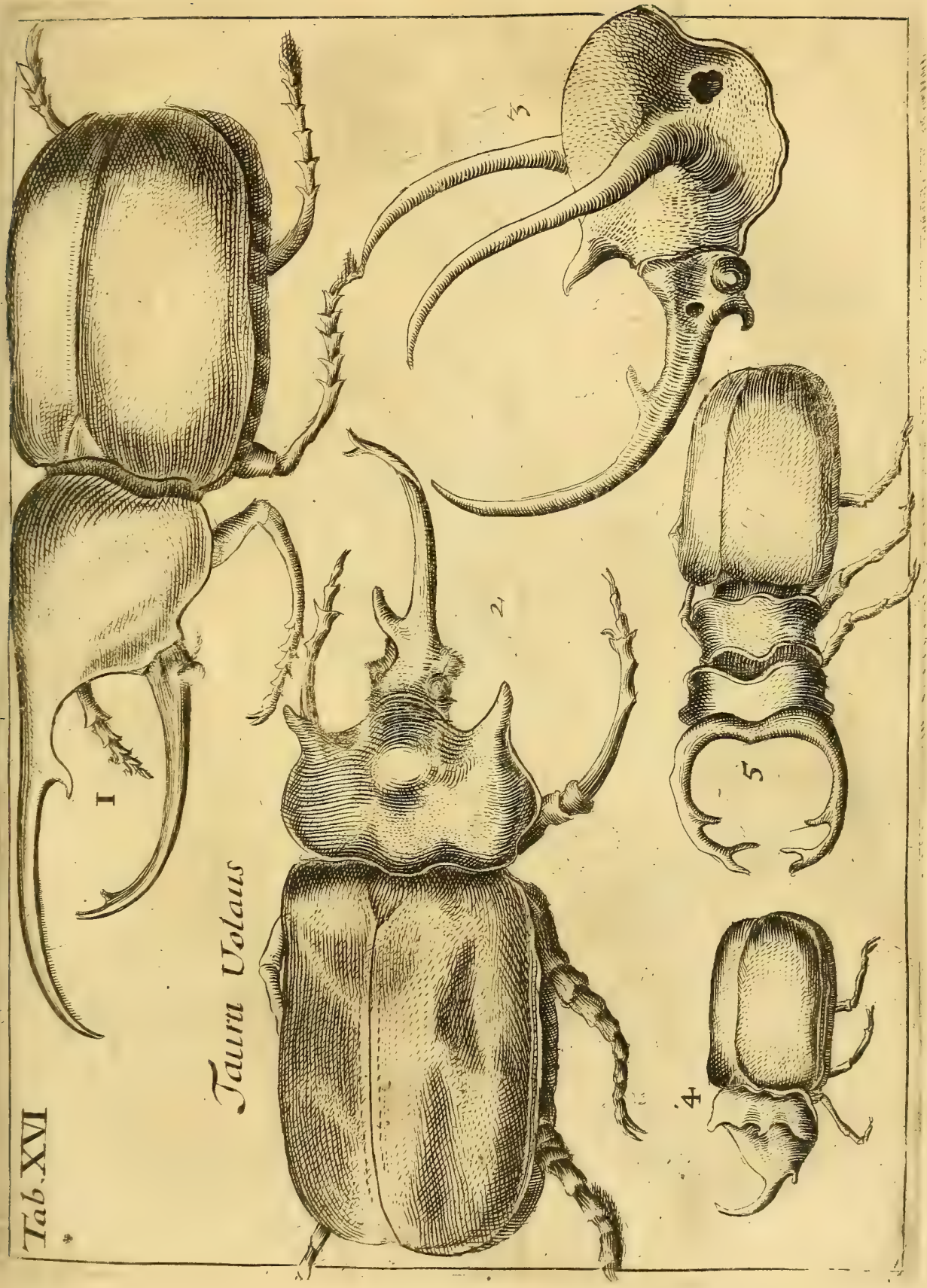





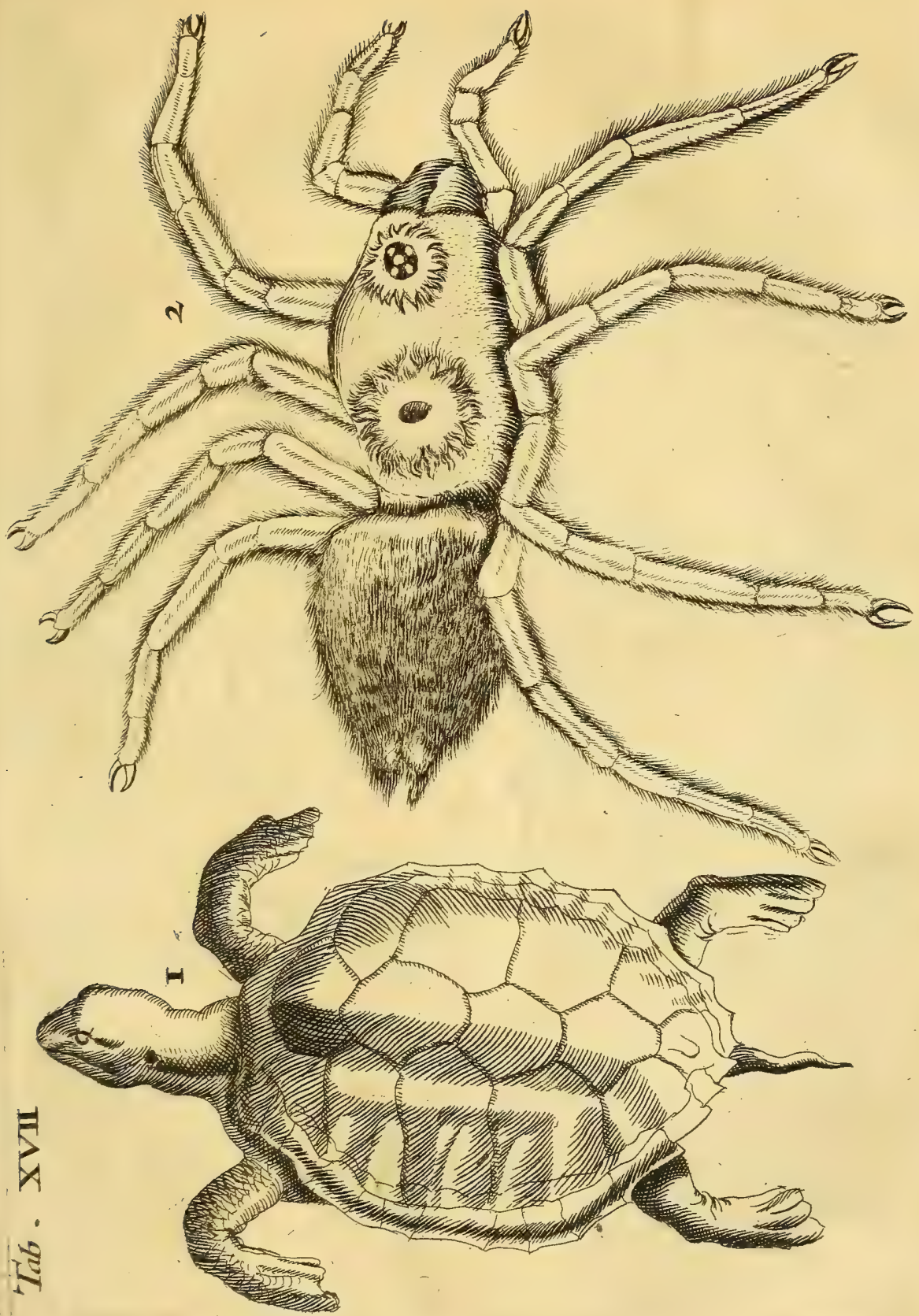



einenigat fremboen Dogel / weld fen Aldrovandus avem Rhinocerotem nennet/ toeil et als ein Rhinocer Sbiet ein Sorn auff Der SRafen bat. SOir baben ber Sdonabel stoeen / feuno bobl unto ganf leidot / gleidjwie Deg. Toucans Tab. XIII. gft aud gebl / nut Dafer oben mit ein wenig totb betmis fót : Das Dbertbeil Des Safnabels if I 12 . Boll lang unb 3. breit / Das Soln aber sben Darauff faft einer Fauft dis cfe. Hefychius uno Varinus forteis ben / Daf in Ethiopia grofle Dógel/ mit Sorrneun auff bem Sotnabel fino/ Die vierfuffig waren/ uno wurben $\mathrm{Rhi}$ nocerotes genant/ und Plinius lib. 10. c. 49. Solinus wie auch Pompon: Mella : Dafin Gnoien uno Ethiopia foldoe OJogel $/$ Die fie Tragopanades nennen / fo groffer als bie follen. Do biefer 20 gel auch oer fern fol/Deffen Scaliger Exer, CCXXXI. 5.6. gebencfet / stueiffelt Aldrovandus Ornith,1.12, c. 20.

Sin folder 20 gel foll Anno ChriAti 499. als bie Ef friften mit Dem Str" clen im Seeftreit begriffen / in ber \&ufft gefeben/und bey Naupactum erfdoffen worben fenti.

\section{TABULA XVI.}

Diefe saffel bailt infich die grofen geboumeten Scarabæos Doder Reffer / fo alle nach Der rechten (Sroffe gezeid net. Nam, I. mirt Scarabaus Buceros
Naficornis genant. Num. 2, Taurus Volans beym Aldrovando. Dies fer nit ores Fleinen Soornern ift unbes Fandt/uno wito weber som Aldrovando nodf Jonftonio de Infectis etwas Nactricht grgeten. Num. 5. miro ScarabæusCervinus Şirfdy Seffer genant'

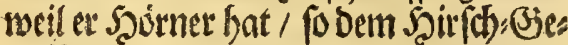
weilse mit bielen Enden ehnlids frefset.

\section{T ABULA XVII.}

Num, I. Eine Sthilopabde doek Schild frote. Derfelben Schalen ober Getháufe baben wir unterfthieblicher Eleine / mittelmáffige unt gar groffe. Die groften Schalen ober Schilbe / fo fie auff Dem গiucfen baben/ ferno nach Der \&änge funfftethalb / bie Sireite abee

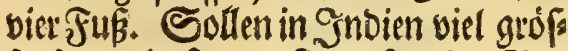
fer fern / Das man fie an ftatt Der Sbos the/mituber'sufabren/gebrauchet / und auff Der Snfel Taprobana ober Sumatra fo gró / Dafi bie Einnofiner ibre Saáufer mit becfen / unb eine bie gankse Şutte bebecfet/ auch ettidse an ftat Der Srbilloe gebraudbet wetben / weil fie fo

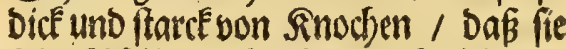
Esine W feile burchlaffen / audh nicht gers bredgen / menn ein 2 sagendartiber fábs ret. 2im 2lrabifchen Seeftrande for len fie auff Dem \&anbe bifineilen im Sande vom 23 ino begraben liegen als Scuigel / und wenm man fid) brauff feset/ einen fort tragen Éónnen. 
Gesnerus ex Plinio lib. 2. c. 4. red)t beridtet. Etlidbe leben im füfien $\mathscr{W}$ afe fer/ etlicye nur auf Dem \&ande / uno an wuiften Sertern / etlicbe im falsichtem Waffer uno in ber See. 2llle aber/fo in $23 a f f e r n$ unb auff Dem sande leben/ legen ihre Fyer / woraus fie erseuget nerden/ auff Das burze Eroreich in Den Sano / Dasfie bie Sonne ausblutet. Wir baber auff unfer Werfiamifden Sieife in Der Seevbe Mogan an Dem Zach Balharu viel angettoffen / meldye ibre Eyer an abgeriffenem ufer in swey orey) Sobritte bom 20 affer / uno uff șugeln ferme vom 20 affer geleget bat: tew/und jtoar aus fonderlicher Intrieb ober gleichfam Slugheit Der Natur/alle verfus auftrum gegen Den Mittag/ Das mit Die Sonne Defto beffer wircfen Ean. YBie biebon in Der Derfian. Sieifebe: fdreibumg pag. 449. ift beridjtet wor: Den. 2Bir haten von berielben Sdjilds: Eruten gegeffen/ Dašfleifo bat einen gu: ten (Jiefdmad/gleid) wiesuinerFleifd)/ foll gefuns fenn/feift uno farce machen/ uno wiro von etticjen fuir \&ecterbisgen gebalten/follen auch wider Ssiffit bienen. Plin. d. l. Sgr 2 lut ift ein gewiffes remedium für bie fd wete Noth. SGre Speife if in Den fruchtbaren Feloern bie Erbfrudite/ item Sdonecten/OSBur me/auds wie Gesnerus nill / leben fie bom Yau. DeB 2 Binters f farzen fie (ich) in bie Eroe/und erfjalten fít bon ber Seudtigteit ber croen.
Tob mus fierben er jeblen/wie mir es mit einer Scbilbetroten ergangen / wel

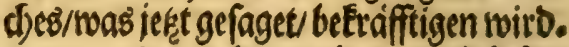
Anno 1653, wito meiner gnábigiten Serz/chaffi auff(Jottorf eine Schilos trote gebracht / poein wenig tuber eine Diertel sillelang / Eam aus Şolland. Selbige fafzete id) in meinen (S)arten/bato te fie aber úber vier sage nicht/Darwaz fie berlobren. Fin Jabr bernach finoetfie cin Daur aufi Dem Feloe in einer Biru ben / bringet fie wieber nach Bottorff. Uno als id felbige wieber betam / bobs rete id ein \&od) auff bie rechte Seite Det Sdjalen / hand fie mit einem Segel garn an Den $\mathfrak{Z a u m ~ / ~ b e f i e l t ~ f i e ~ a b e e ~}$ Eaum fechs 2 Bochen/ in warender Zeit Eunte ic) nicht mercten / Dap fie einige Sirciuter abgefreffen batte. $\mathfrak{A}$ (I) fie nun wieber aus bem Siarten gefommen/uno Das Segelgarn verlaffen / hatte ich fers ner Eeine Gjedancét mebr Darauff. Sechs Jafir bermach aber nemblich Anno 1660. im Sitiegsivefen bat unfer Commendant auff 30 ttorf 5 . Francifcus Sungheim micheinft zum Jtúbs ftucfeauff ein gar delicat Effen/ fo ic in biefent \&anbe wol nicht finden wutes be/eingelaben / wolte aber nicht fagen was es waire/war ein Elein (3)erichte/wol sugerichtet / nur Daß es etwas bartlich war. Alls bie Mablzeit gef beben/frags te er / ob ich wuifte / wobon wir gegeffem/ und lief Das Schilb bon ber Sóbilds trote auff ben Sifd bringen/ Da ertante 


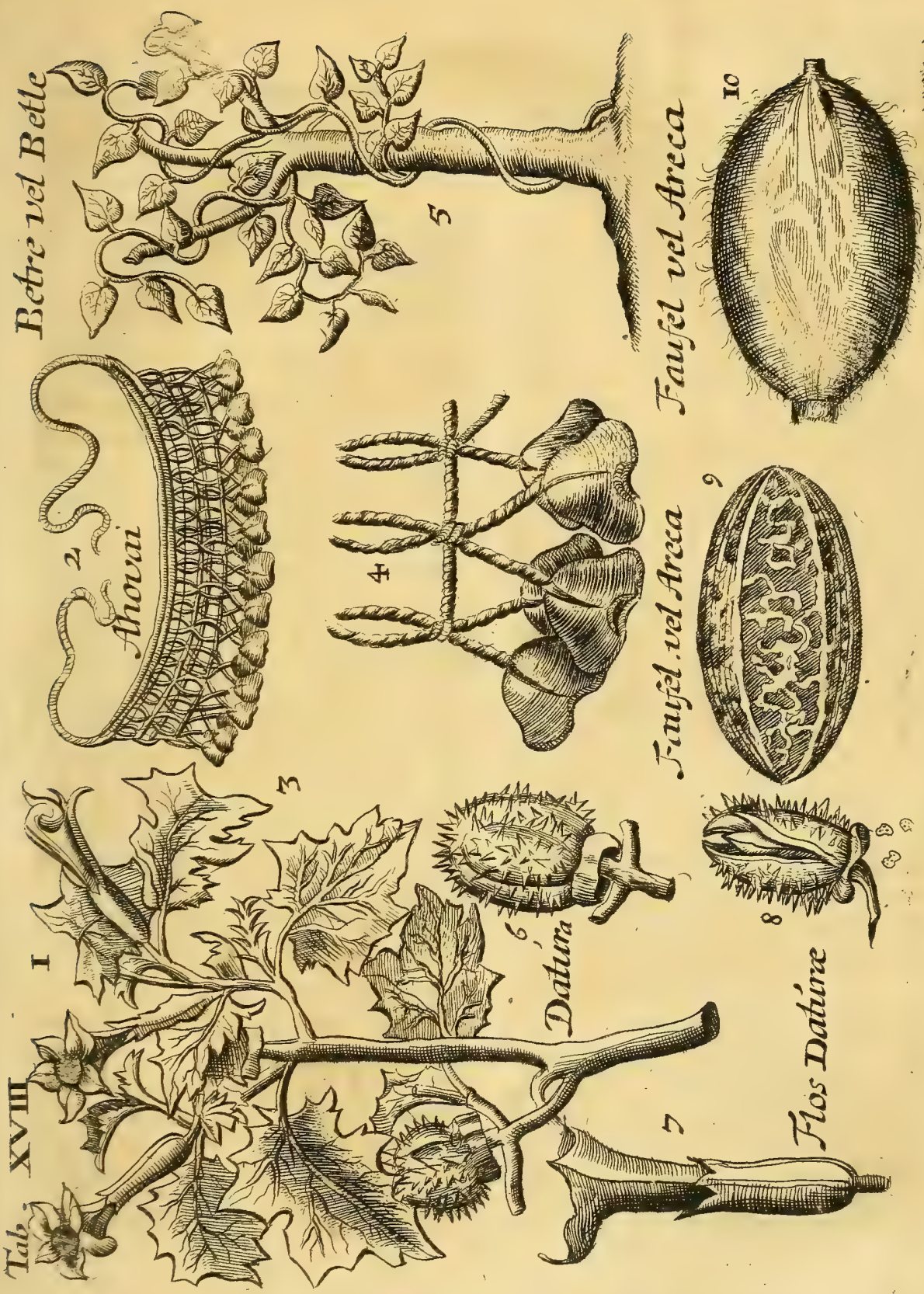



id) am Durchgebobrten \&ocbe / Daß es meine/ fo id vor fects Jabren werlobren batte/getwefen. SAllo mubtmaffee ich/ Daj fie muifen rofcido humore, wie Plinius von Denen in fitientibus terris Africe foretibet/oom Shau / von Der Erden uno23urtmen ibreltinterbaltung baben.

Num,2. Silt eine Spinne aus $\mathfrak{B t a}$ filien nach ih rer rectsen Giróffe und Sses fialt abgejeidgnet. Die Einmolyner nennen fie Nahmdu Gaucu, fie fol fu getwiffen Beiten iffere Şaut abwerffen/ mie bieSd)langen. Sic ernetren fich von Fliegen und anderm Bjefómeiffer follen lange-leben / Marckgravius fprift in feiner Sçiftor. Brafilienfi, baß er eine swey Safhe in einer Sdjadjtel ohne Speife lehendig erbalten. Mo-

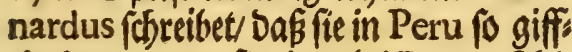
tig fenno/wenn fie einen beifen/ unfebs bar fterben múffe'/ menn ibm nidbtalz:s

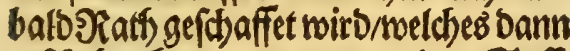
gefchehen Ean I wenn man Den Saffit von Feigenblättern/Fo ba bäuffig allents balben fethen / in Die 23 unde trieffet. Monard. c.62.

\section{T ABULA XVIII.}

Num. I. 3.6.7.8. Sit Die Datura Indica, Staube / Sblume und Frucht' wito audh forft Dutroa genant / Det Sarme wir in Snbien von ztweverley seuten gemigbrauchet/nemblich von ben geilen 250 cibern und Dann von Dice ben/ ober ungetreuen Dienern im Shaus pe/ bann ber ienige / weldsem ber Ẽas me Datura in Effen DDer Irincten if bengebradstworben / wiro auffetlidse Stunben fénes Dertandes beraubety Daj / ofer fchonwachet / von nictsts meiß / unterbeffen Eónnen Die Stauen wie auch Die Diebe in Bjegentwart bes Mannes ibre \&uft uno 22sillen haben/ uno wann Der MRann ertwachet / meynet er/ er babe nur einen fúffen Schlaff ges than. 223ie bierbon weitlaufftig in

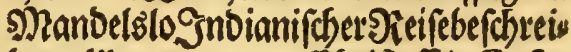
bung lib. 2. cap. 7. Éfriltoffi à Cofta meynet / Dapez eine 2 Irt von Der Stramonea (en). Es mitb biefer Saame auch in Seutfoslano gepflanket uno reiff / maffen es Der BSottorffich be \&ufts gártmer Meifter Bjabriel ₹ater / ein fleiffiger unb erfabrner Mreifter / fo fíds bemulbet allertey) rare exotica berzu bu fohaffen und zu pflanken / Gat er bifis ber faft Sábrlich / wenn Der Sommer nicft zu Ealt/ reiff beEommen/ ob eş aber

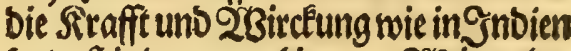
bat / frumbe zu probliren. 2raare aber nidjt gut für unfer \&ano.

Num. 2, und 4. feund Sdjalen von einer Snbianifchen orrucht / Deffen Saum Ahoai bon ibnen genant / foll fo gró als ein Birnbaum fern / bie Fruchtift in Eaftanien Biróffe. Uno foll ber SBaum in ben wuffen 2 bálbern gefunber/ Ind Die Fructituon ben Ein Dii 
mofnern furt Den 21uplaindern in ges beingeforlten wetben. Dann Mann uno 23 eib/wann fie cinander gram wer: Den/ follen Die Frudt pulberifitet entwes Der in Soback ober Speip uno StancF verunif bet benbringen. Die Schas Ien/ fo Die Frudjt umbgeben / merben auffgefdyitten/geborzet unb etwas ge: branot / fo Elingen fie alo Scbellen. Soldhe binden bie 2 Bilben umb Die Strme und ßeine / wenn fie tanken und luftig fpringen wollen. Saiervon fich reibet Clufius in Exoticis pag.232, und Pifo lib.3. de Venenis.

Num.5. Iftein Siraut/Das bieSp Gndianer Betele, Betre tno aud Pam nennen/ wie \$zlandelslo fótreibet/ Die grumen Rblatter / fo lainglicht feryno/ werben Datbon nur genoffen/o ag Siraut bat einet fot wadjen Stengel/gleich wie Die Sebera / Datumb mus fie an einem Stuck auffigeleitet merben/ foll an Rraut uno Stengetn Den $\mathfrak{P f e f e r : ~ S t e n g e l n ~}$ nictyt ungleich feun/ fo fich an eittem an Dern Stammanfitten. Utno weil es alleine nidht/ fonbern mit einer Frudbt/ fo fie A reca nenmen/gebraudst wito / fo leiten fie es an einem foldben $\mathfrak{B a u m}$ bin. auff / Damit fie beyoes bey fammen ba: ben. 2on Diefem Betre bat Clufius lib.I. cap. 18. auffúfylid gefdrieben/ welches luftig ulefen.

Die Núfte num. 9. fenno Den Mu featen Plúfen nicht unehnlich / baben aber einen umangenefmen Sd bmad und sufammentiebende Sirafft / Dabet bált man es wiber Den Sdforbod gut fu fenn/wacbien gleich mie bie Sockers Nuiffe an Sáumen / baben auch fo ldse raube Sdbalen / wiefelbige num. 10. zufehen. Avicenna nennet fie Fuffel fonft Faufel genant. Sgievon bat Clufius lib.I. Arom cap.25. eine ausfitibts liche $\mathfrak{B e f f h r e i b u n g / ~ p o ~ w o l ~ j u l e f e n . ~ E s ~}$ wiro aler bevbes mit Salcé / fo aus Muffe: Schalen getrandt/gefauet/ fo gibt es̉ einen rothen Saffi / bavon ifhnen Die sippen gants roth rerben. Mann uno 2 Beiber gebrauchen fich oeffelben fonberlich bie Jortugifif ben frauen in (S) a/moie bierson weitláuftig uno aufs fúbrlicher in Mandels!o Snbianifher Sieifebeforrcibung lib.1. cap.24. zu les fen. Item Clufius lib. aromat,6. Garcix p.275. \& 288.

\section{TABULA XIX.}

Num. 1. Sift eine 2Imericanifde Fruct) , Clufius in exoticis lib, 2 . cap.6. (chreibet/ Dap fie audh in Guyana wadbfe / foll eine Iirt bon Drandeln fern / unb auff langen fomalen Bius men I fo wie bie Fidsten auffiteigen wachien. Die Schale ift ohen voller Jockeln / uno fo bart nlo bie oy firficts. Sernen/Die Rern aber Darein ift als eine Niere formiret / foll einen Mandele (3)es fosmace baben/menn fiefrifich ift. In fere/fo alt ift/lajfet fich fobneiben unb ans feben 


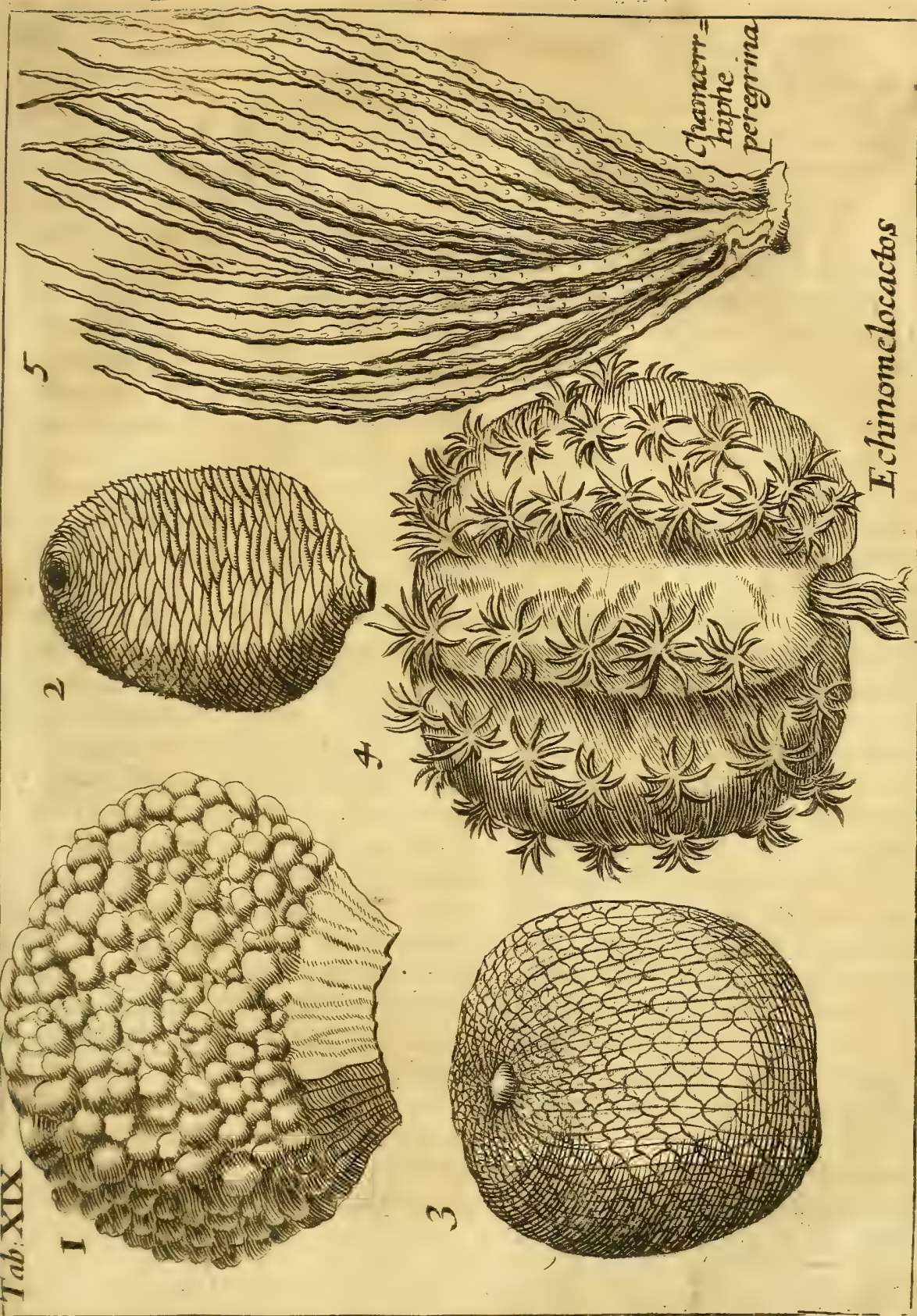



fefen wie bie Sern in Der CodferNuf. Vide Johan de Laet. in defcript. Americre lib. 9. cap. 4 .

Num. 2. Sft ein 21pffet vom Ce Dern:3aum/ ro vom Berg Libano ges fommen. Sfit mir vom Scerzn Jacobo Golio Oriental. linguarum Profeffore zu \&enden/meinem gutenfreuns De gegeben / und Darbey berichtet wor: Den/Dapibm/ fein 23rtuder/ Der auffoem Libano in einem Silofer wohne / fellhs genneben anoern fremsoen Biewadifen zugefdicatt. Lobelius p. 488. beforreis bet Diefen ßaum mit mefrem.

Num.3. Sifteine fremboe Frudbt/ fo grof als ein 2ipfel / foll auch auff groffen ßáumen wad) fen / fommt aus Guyana in America gelegen/ fat eine gar barte Schale braunroth / und wes gen ber orbentlicten Sirctelsinien / fo eg̉ gar proportionirlić) Die lánge und fobrabe bált/ anmuthig anjufêten / ba eह Clufius Daber ein miraculum na. turæ, ein 28 unberwercef ber ग?atur nennet Exot. lib. 2. cap.4. No uf eine groffe Palpam ober innerlictse פalfe baben / fo man an Dem Slappern met: céen Ean. Şabe die Schale / umb Den

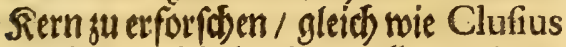
Den feinen/nicbe brechen wodlen/weil Der 2ipffel fobon / uns wir nur cinen bas ben.

Num.4. Iff gar eine nounberfellgas

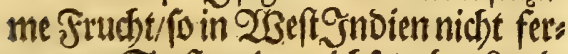
ne bom Seeftrande mogidfet/obne \&aub/ bat nur eine 283 ur'sel als ein Elein Fin: get Dicle/Lobelius unb Pinade, Diefie ausfúbtlich befchreiben / nennen fie Ecchinomelocaeton ober Melocarduum Echinatum, weil es fo ftachlichs alsein Jgel ober Diffeltepff. Das Corptsilt mie eine mittelmaifige Mes lone/mno bie Sterne fo ordentlich het: umb firgen / fenno fo bart wie die Dors nen. Die Einnoofnet follen fie wie bie s)elonen effen. In Sarben/wents fe frifob / follen Den Eucummern oder 2irurten gleich Feyn. OXsin Gaben Der: fellen stweene/ Die eine if t nods gang/und etwas fpiser oben zu /als hier abgebils Det/ bie antere if 2llters baller gar gets fallew/ fo biefer efnticher genvefen. Lobelius fifrribet pag. 373. Stirpium adverf, nova: Quem non juvat in hoc miræ raritatis \& venuftatis Carduo folertem naturæe opulentiam contemplari, is fe putet averfo natam ingenio à lautiore Philofophia. $2 B$ er diefe peltrame uno von Patur reis

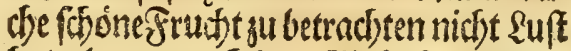
bat / hat gants Eeinen Philofophi(d)en Fopff und Elugen Werftano. Clufius in exotic. bef(d)reibet audf Dergleiden Melonen/ welche mie eines Surrcéen Dinike geftalt. l.r.c.24.

Num.5. Diefer Strauth wirb vom Lobiliouno Clufio Chamærrshiphes genant/ werden auch son Denfelben bes pobrieben. IIn Den Neifern fiefert man Die Veftigia , no die Blumen doer Di iii Sruidste 
Fruichte geferfen / follen auffoen Sinfeln unb bohen filippen/ /o in ber See ans zutreffen/wads) pen/mie man dem Clufio beridtet f fat. Lobilius meınet in Stas lien in locis maritimis gebrauche man es an ftat Der Refyebefen.

\section{TABULA XX.}

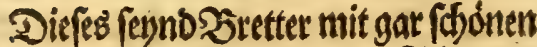
Jierlichen Streiffen/210ern / PBlumen/ gleid man in etlichen 23 allnuss sooer

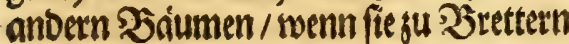
gefónitten uno geffobelt feenn/ fibet bon Farben braun / peyno aber von Eeinem 23aum/ fondern wirb aus ciner mineralifhen ErDen gegraben/ Daber es von Francesco Stelluti, Der in Italica lingua einen gankęen Tractat mit fois guren barbon außgęhen laffen/Lignum fosfile minerale genant wirt/und weil es gar ein merceflid) und feltam $25 e r d "$ Der Natur / uno meines 2 Siffens vor Diefem Autore Eeiner ausfribrliç Daks bon gefdrtieben / will id) Dem \&efer uno

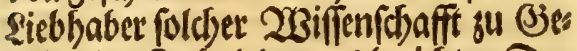
fallen Den Inhalt Daraus berichten. DeE situl Diefer 2 ucbsift:

Tratiato ded legno fosfile mi. nerale nouvamente fcoperto, nel qusle brevemente fi accenna la varia of mutabil natura di detto legno rapprefentatovi con alcune figure che monftrano il luogo dove nafce, la diverfità dell' onde che in effo:fo vedono, e le fue cofi varie, e maravigiliofe forme. Bu Rom ge: Drucft An, 1637.

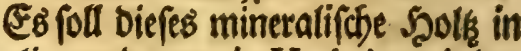
Stalien uno jwat in Umbria, inder Segeno Todi an verfobiebenen Drtew iebod) am meiften swif then Collofecco uno Rofaro madfen / von Duc. Frid. Cefi di Aquafporta erkt gefunden wors Den. Uno foll in eimer Ereioigten Erbe liegen / tweldye allgemad) fo wol burd Die Shire ignis fubterranei ober unter: Erbifbem Feur ( Dann bie Erbe gibt bafelbft einen Dunft und Faudy / aud bismeilen Flammen von fidh/fonderlich

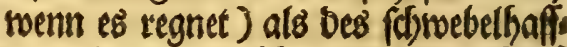
ten uno mineralifoten 2 Baffer:. Dafs

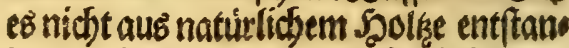
ben / gilte Det Autor Diefe Urfachen; weil eš r. Eeine 2tefte/Zneige ober223ur: keln wic andere Riáume bat / fonbern nur wie ein gleiffer Slok ober Sttumpf 2. nicht auffgericht / fondern in ber (Ex be auBgeftrectet lieget / 3. Die 21Derm of Der Striche foes bat / feyno nicbt alle unb úberall in gleicher Sseftalt / ponvern veridsiebentich gebilbet/ balo lang uno gleich/ balb Erum/ balt enge/ balo breit/ balo Sळlangenmeife/ ba andere 3 aiu me am freiffen auffwerts uno gleids ges beni/ woourch fie ibte Nabzung an fich jiefen. 4. Er bat andere ftucten șols Dafelbft / fo von ber Erben befallen ges wefen/ uno lange barin gelegen/ aufiges grabew aber foldse 2iefibaffenbeit/ als 

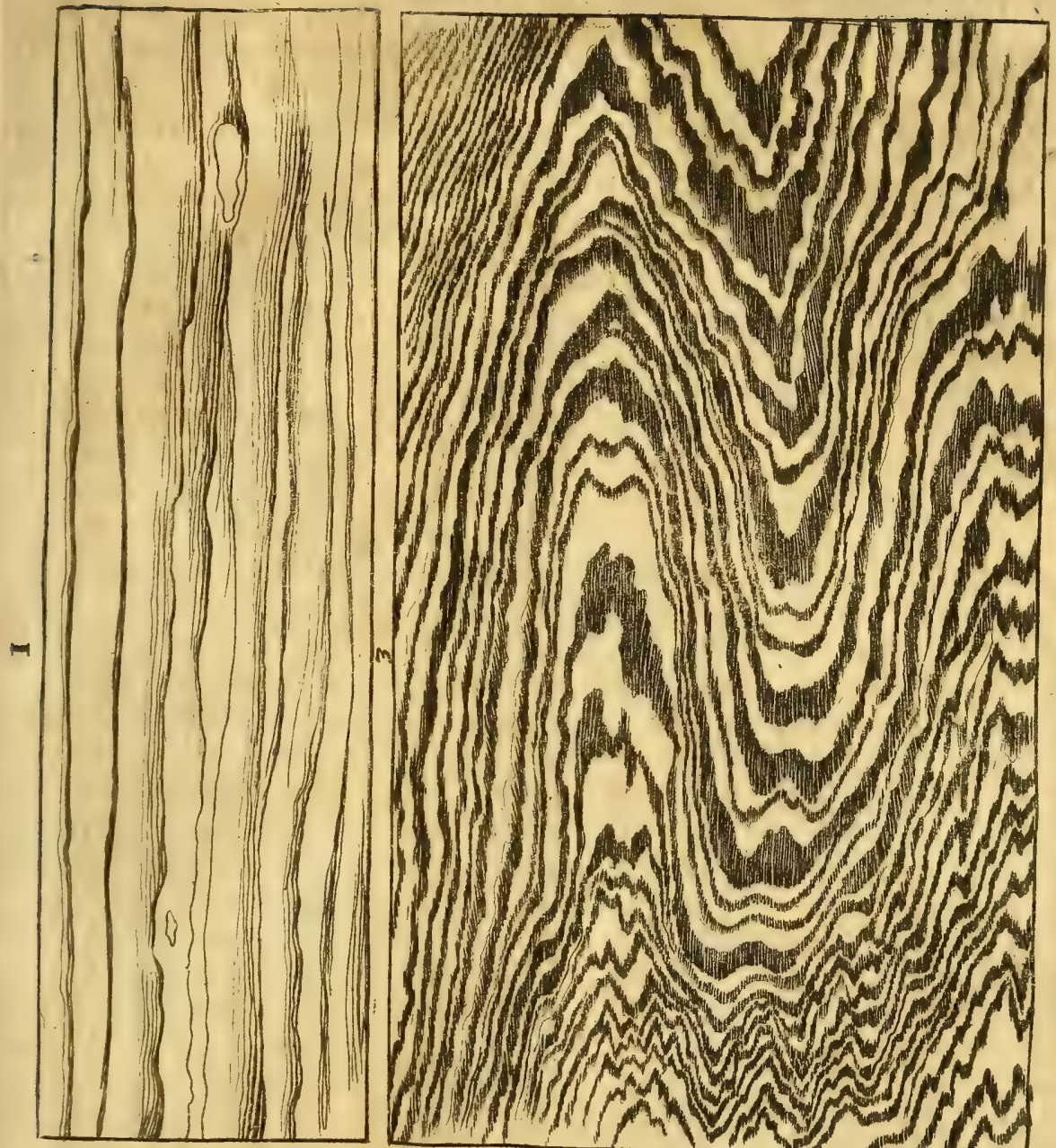

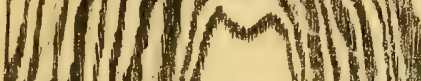

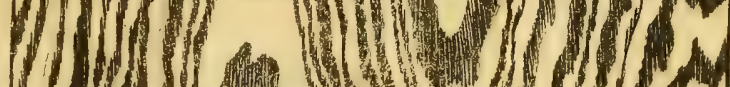

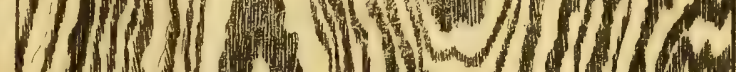

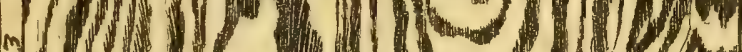
m

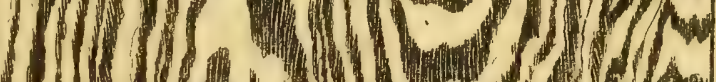

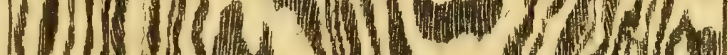

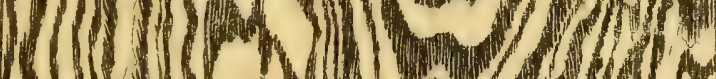

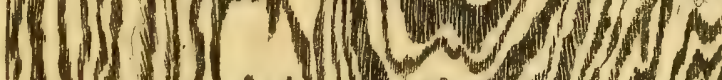

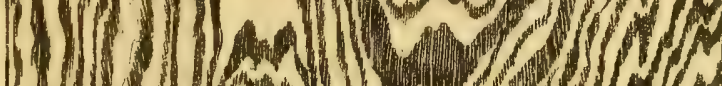

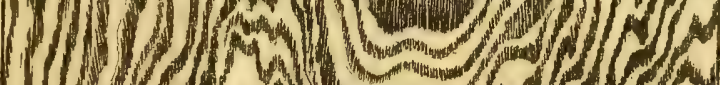
(1)

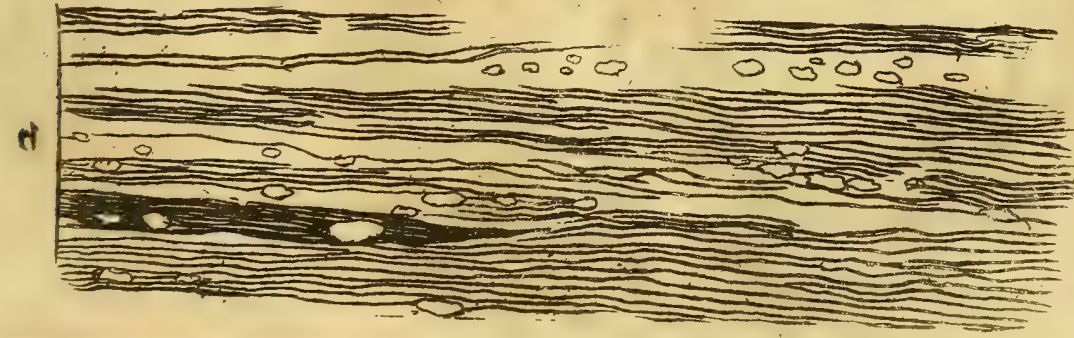



bif lignum minerale an fich bat/nicht befunoen. 5. $25 \mathrm{senn}$ man worafer leget / fo fofmimmet es nicht

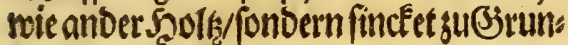
De/mietrol es fich fonfen faneiden/ bos beln uno hanotbieren láffet als ander Şol babt/ Daran ein Sheilnoch rectete Sreios Erve/ein Sbeil allbereit fu Solk / uno ein Sheil wie Roblen gefehen. 7. Fin Det man in etlichen foldben flucten S马ol nod) treiffe Sơrner / fo nod) nidft gan ins Sols verwandelt worben. 8.9)Ran

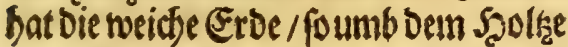
getwe fen/abgenommen / und in ein Bses madj Des serergogen von Cefi zuA quasporta geleget/meldye nach etlidserishor naten in fold) Şoles yertwandelt wordent nit groffer Verwunberung aller bie eg gefehen / Daher gar nicht ju stweiffeln/ baß bie Erbe Semen und materia dies fer Saltaes fen. 9. Es hefinben fid Die 2̧lócfe Doer gankse Stuilen nicht einerley Irt von formen / eftictse lang/ etliche turf/ ctliche Enlindriforer/etliche

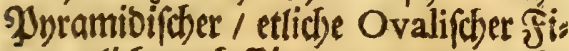
gur/etliche auch (Eircular / unb etliche balb Circfelstumbe. 2in eftlid)en ift Dab eufferfte bolpericts unb sectorechlich/ in eflichen fobrwats wie Siohlen. 10.

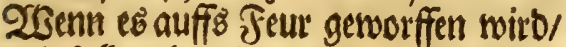
uno foll verbranot werben / gibt es Eeine Flammen / fonoern glimmet burd wie anoere Foblen/ foll aber groffe Szige ges ben/ und Dag Feur in ber 2A chen langer balten/als fontzandere Foblen. Mran finoet auch Dafelbft etliche Stricte / fo balb su Stein worben / tmo die anber Şelffte J̧ols Glieten / unb alfo lignopetra ware ju nennet.

$23 e n n$ man nun biefe zelyen anges fübret Urifadjen will beobad)ten/ pl lafie idfs bem judicio Des Seferg beingefet:let fenn/ wag er von Athanafii Kircheri Mersnung bon ber generation Diefer Scolleses falte / wenn er in feinem Mundo fubterraneo lib. 8. fec, 2. cap. 6 . faget : Dico olim halce fuiffe arbo* res, cujus figna pervetifti cortices fat fuperque demonftrant, in fodina ubique rasfim obvias, non tamen fucco aliquo lapidifico in petram induratas. Sed fucco aliquo peracri \& mollificante in minutisfimas partes diffolutas, quibus terreftre lutum, cujuscunque tandem id generis fuerit intermixtum ac inter. fluum particulas minimas arborum jam diffolutarum in unam maffam terro - ligneam contraxerit. N(t)y net alfo/baf ber Utrfprung aus alten vers mobderten Báumen fey / mit welden fich eine irzoifche materia bermifche: aber to muirden Die Figuren nidst fo fchón proportionirlich und beutlich ju Feben ferym/mie Die Abbilbung / fo recht nad) Dem Shol/se geconter feitet/anbeutet. So mirbe aud bie weidbe Erbe/ fo bon

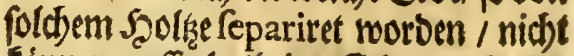
Eonnen aufferbalb Der Eroen ju Şolge rerden. 


\section{T ABULA XXI.}

Num, 1,2.3. Seuno Gloffopetrax, wie fie Cardanus nennet/ oo be wie mane in gemein Dafúr gebalten bat ; Natter unb Sdlangen Bungen / fo in Stein verwandelt worben. Itno weil fie am metilen auff Der Jufel M) alta / oon wels chet bie Unferigen gebracbt worben / ges funden werben / uno fonft Eane leberbi: ge Sd) langen Dafelbit befind licb. Mgey: nen fie/ Daper Daber Eormme; weil Dem

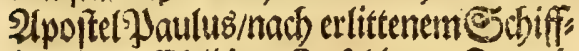
brucbe auff felbiger Snfel beym Fent ei: ne Stter an bie Şand gefabren / und ins Jeur gefd)leubert worben / wairen Daburd alle Sdblangen uno Detern vertilget uno ju Strin worben. Flbet man fiebetwol/ Daf es feiner Sdblan gen ober Stter: Zungen/ wiefie in Europa fallen/ efolich. Ftlidje meynen/ Das es eine Ult von Donnerfteinen fern fol. Sie feynd alfier in ifber redten Broffe geseidfnet. Es feyno Derer anevertey) Art / Die Eleinen / (gleich wie Die groffern) etliche weisgebl/etliche aber fobroarsblau. Olaus Dormmennet/ Dafis etlidbe follen Bábne feun aus Dem Fifche Carcharia, uno gu Steine ge worben. Sb wir num zwat eben fols dye Zábne/ soie bie Eleinen/ nods im Sias d)en eines Carchariæ fiken baben / fo fieber man Dod, / Dap beidoerley Art in Der Eroen wadsfen / uno baben nod theils ibte SNutter an fid . Wuic aber folches zugebe/ Daf ctliche Vagitabilia in Stein verwandelt werden Eonnen/ bat 5 . Kircherus in mundo fubt. 1.8. de facultate petrifica glaubmurbige Difcurfe.

Man foll Dergleichen Stein Zungen bev) Suneburg in Den Allaun Siruben wie Anshelmus Boetius auch bey?nts werpen / wie Goropius Becanus mels Dern/ viel finden. Thevet. forreiber/ Dafer eine foldse Zunge bey einem bals ben Fuplang gébabt / uno Dem Gesnero zugefdicte/ Defien autd Gesnerus de Pifcib. 1. 4. Thevet, Cosmogr. 1. 1o. c.7.gebencêt. Es tragen etliche foldse Zunger in (jolb uno Silber eine gefaffet am Scalfe oder fonften an fich/ uno meynen , Daf es roiber oie Baubes rey und (siffit bientich fey / und wenn ce. ouSifft Eomont/ foll er fómiscent. Dis babe id mit Boetio nidjt befunben. YOas mefor für sugeno biefer Stein bat/Ean man beym Bartholino lefen.

Num. 4. Serno Steiniein vor feibiger materi in Grơffe uno Form wie fie geseichnet fteben/ werben bey Den Sungengefundern/ welche fie Dtter:?21 gen nennen/uno faft gróffere Srafft als ben Zungen suftoreiben / wie mein

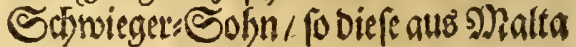
getbracht/ berichtet.

Num.5.6. Sennd Steine / Brontia ober Ombria Donnersund $2 B$ etter: fteine genant. Damn etlidge finv in bee NRese 



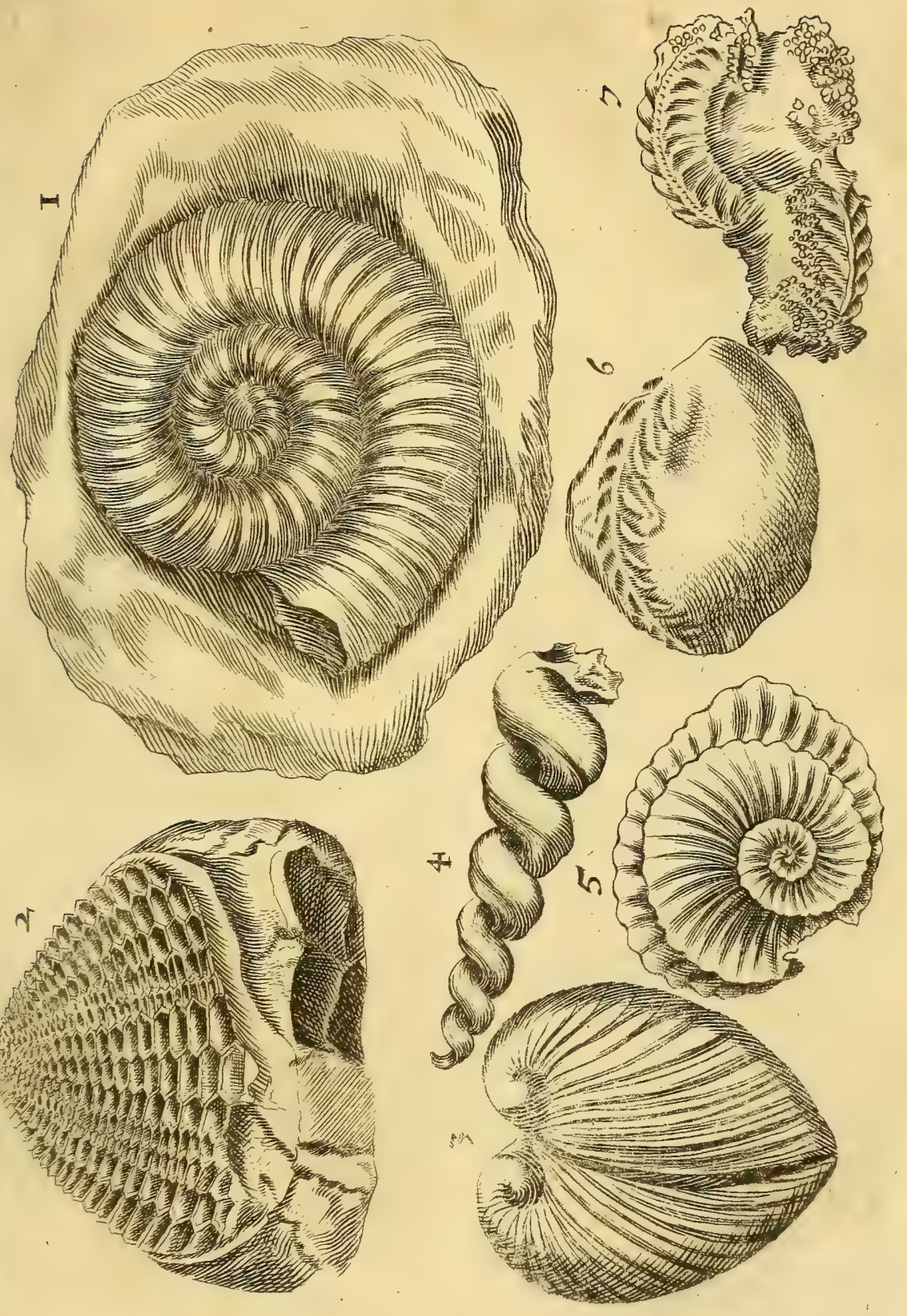



2)ennung/oaß̧ felbige im Donner ober ftarcfen PJlaģregen aus ben 200 oldén fallen. Estliche nennen fie Schlangens Ever/ wieaudb Frotem|teine/Daß fie von Schlangen uno Rroten:Speichel uno Echaum follen jufammen getwitcfet feyn. Plin.lib,29. c.3. Boetius lib.2. c. 24. will fie Cheloniten nennen. Nian fintet fie an unterfociedlicben Deten / fonberlich follen fie in Dennes maref bey Dem 2doelichen Bsute Sorm brup háuffig liegen. Sch babe aud stoes ne ben ber Fetung (3ottorff am 3ergel Den S.F. D. umb ben 28 all Darmit a 4 . verhóhen/abtragen laffen / gefunden. Sie fenno alle balbrund/uno baben uns ten am Sianbe eine runbe platte Stettel als eine Erbo gro / / gleich als wenn fie Dafelbft an einer Yrsurkel gefeffen. Mart bált Darfuir/Dap foldse Steine / wenn man fie bey fich trägt/ cinen bor Weftis

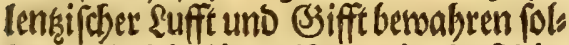
len. Etlicbe bildenifnen ein/baß dies fer Stein/menn er getragen wirb//chlafs" fend machen/audb bie Victorie wiber reinen Feino gu wege bringen foll. Das ber fie ifn in Degens Snopff madien laffen. 2uch foll er für ben Dormer" (c)lag betwafren. Die Sauterweiber in Dennemate" ( tefte Wormio ) balten bafuir / bas er gut siber Baubes rey fery / Daber fie ibn ben ben S) Rilch: Eymern uno Mnilof:CEmmern ju bals tenpflegen.

\section{TABULA XXII.}

Num. r. Sift ald eine 2 tht bon Brontia Doer Donnerfteinen / gar Fels Gam anzufehen/bat bon Elarem weiffen Eteine folcbe Beflen / als menns Der 33ienen 23 erce waire/ in weldser fie bas Şonig tragen / nur Daß biefe bierectft unto ablenglicht als parallogrammata fenno / welche fich nach Der Şóbe Des Steing ju verjungen / Dergleichen audb Olaus 230 orm in feinem Mufreo bers seidgnet und bef(brieben bat.

Num. 2. und 5. peund barte nocis: gelbichte Steine / als ein 2 Siboer scorn in fich getrummet anjupeben / werben Ammonis, Hammonis ober Supiters Cornua genant. Die Egyptier, fo Den Supiter Hammonem nennen / folo len Diejen Stcin unter ifre beften EDele gefteine uno in ibl Sceiligthum ferser. Dann baburch foll man pradivina fomnia baben/ burds roelche man weifs fagen fónne/ wie Plin. lib. 3I. cap. 10. unb aus ibm Boetius cap. 246, fichreis bet. Man findet audb Deb Alexandri Magni SBilonifí auff ber Mnunke (Des rer wir etlicke baben) mit foldbem Ammonis Doer Gupiter: S.orn am Sopffe gejeidjnet/ und Daffelfe Daber / weil Deg Ammonis $\mathfrak{H}$ riefter ausiltner fabrenbeit Der S3riechifónen Spradbe benAlexan. der aljo Barbare angerebet: $\pi \tilde{a} s$ dios (5) 
fili Jovis, wolte aber fagen: Tardíon filiole. Daber wolte Alexander bets nad) für Supiters'Solbn angefefen und geebret feyn. Cxl. Rodig.lib.22 c.20. faget : Unde cæleftis originis captata creditur gratia, cui initia error dederit.

Num. 3. Sft cin Stein Bucardia senant/ weil er eine BSeftalt eimes Odys fen S Serergens bat. Selben foll Fernandus Imperatus lib. 24. cap. 26. etftlich befchrieben baben / wie Olaus 2Sorm uno Johan de Laet, de Gem. \& Lapid. c. 32 . gedencten. 23 ir haben Derferben bier / und ift immer eines an Derś als daś ander formitet.

Num, 4. Semnd Edonecten:Stei: wie / wachfen in cinem múrben Steine báuffig beyfammen/ Der / fo wir haben/

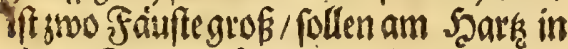
einem 2 erge gefunden toerden.

Num. 6.7. Seino barte blaue Strine/fo fids an ben feiten Dem Cornu Ammonis gleidben/oder Dodb Die Muts ter/an welchen Die Cornua Ammonis ober Dergleidjen Sdjnecken:Steine ges feffen.

\section{TABULA XXIII.}

Sit ein Shrwank von einem Ses 21bler / wie ifn Salvianus uno Bellonius nennen/4. Ellenober 8+ Fus lang. Rondeletius aber faget: Dá auch cin Ipecies Paftinaex, ein Stacheloro den fer / weldser binten im Schrwantel Der auch fefre lang/ einen gifftigen Stor chel baben foll / Daber er von Den Sols

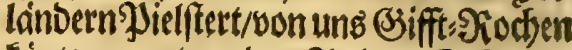
Eónte genant werden.2n dem Sdjwaw Ke aber/ fo wir haben / Ean man Eeinen Stachel / audb nicht Daßa einer Da geme" fen/mercten. Selbiger 2loler foll eis nem Siochen faft elynlich rehen / if wom

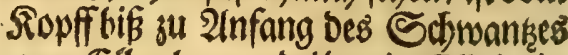
: Jwen Ellen lang uno úber eime Elle breit.

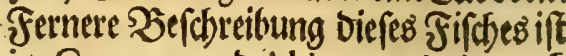
im Gesnero uno Aldrovando Da auch foldh ein Scjwant verseidynet / su finden.

Num. 2. Sfte ein Ropfi won einem Sungen Delffin / meldber von Den ₹eutfchen Meerfobwein/ yon ben Mediterraneis, morim er am meiften ges funden nit' / Becdoye, woil er cinen berwor baungentoen langen Sdynabel gleich einer (3ans bat / genant werben. Aldrov, de Cetis lib. I.c.7 + Uno weil Das S beil Des Ropffes/ no Lar Schnas bet angebet/ eingefoogen / ober wie Plinius lib. 9. c.8. rebet : Quia roftrum habet fimum Simonis nomen omnes miro modo agnofcunt, múgen gerne Das 230 ort Simon forten: Eşift rine 2irt won $2 s$ allfifhen/ hat eine fobmarkse Şaut auff bem Sictéten / man finber fie in allen Meeren / fonderlich im Ponto uns Mare mediterraneo. Anno 1661.ben 27. A uguft, ein folcher Delphin sin 20 ciblein mit einem jun 


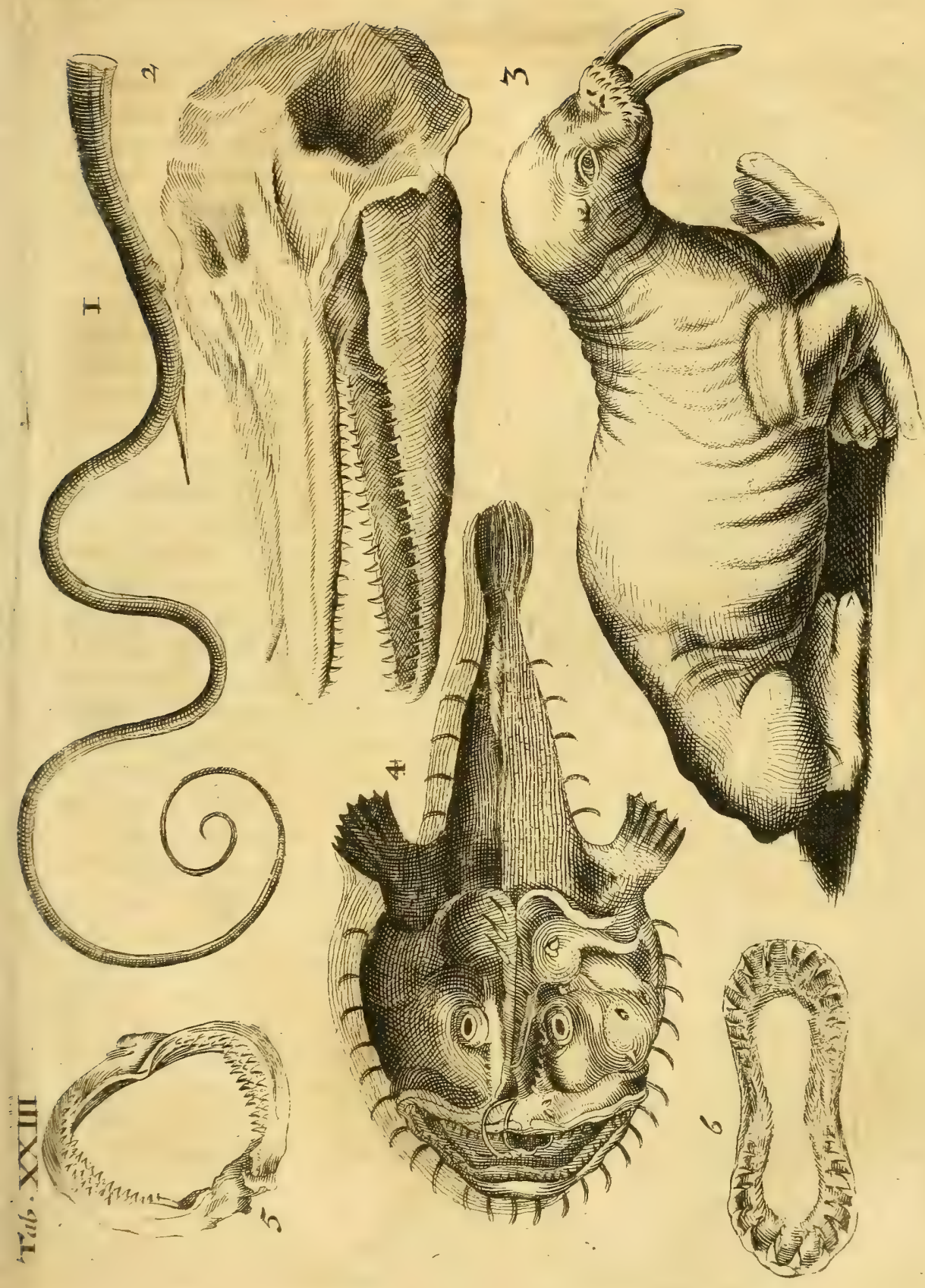



$\therefore \therefore \therefore$

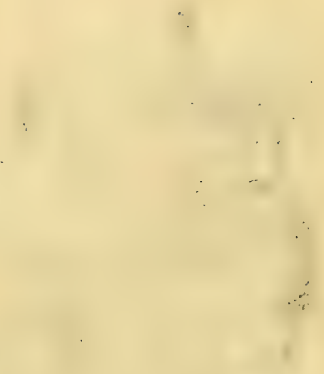


gen Delphine vor Apenrade im Şafen lebendig gefangen morben / 13. Fllen lang uno 7. Ellen dick. So ein graus famer Feino uno Derfolger er ift Der Fiffore/ fo eingrofier siebhaber ifter Der Menfdsen / Dafer fich aud oor fie nidst fobeuet/ fondern gerne bey Den Scbiffen if uno fpielet : foll auch ben todten s) Nenfchen: (So orper gerne ans \&and tra gen/mie Plutarchus bejeuget / Dap fols thes an Des Hefiodi oon Den Mreers Siáubern er foslagenen corper fie follen erviefen uno ad R hium uno Molycriam getragen baben. Er foll gefobnin: Der alsein Wogel / ia alb ein P feil Dar: bon fobieffen / und ein pebr forarfises fidte haben/ foll 300. Jabr/wie Die / fo im Nilo fich halten/ alt werben Eönnen. Er foll Eläglich uno jámmerlich win feln/wenn er gefangen miro / uno follen ifnoie Fifcher aus Motitleioen / fonbers Iid) weil er cin Nlenfidsen:Freund / nicbt gerne fangen/Aldrov.d. I. Er foll aud ein groffer siebbaber Der sonfic fern/ weldes Plinius uno bor ifm viel andes re Scribenten begeugen. Daher balte id) / fen Die Sciftoria won bem Mufican ten Arion ( 10 ferne es eine warbaffige (jefchidft ) Dafi er Durd Den Delphin auffgefangen uno gu Rande gebradjt worben (el).

Die Siftorie aber wiro alpo erseblet beym Plutarcho in Conviv. : 216 ber (Snther fds!äger A rion aus Stalien uiber Die See zur Stad Tænarum fafren

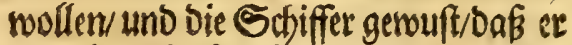
mit feiner Evther ziemlich Sielo verbies net/ Gaben fie/ umb Die PJ fennige zu be

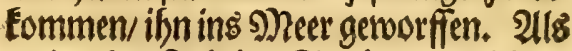
Arion Der Sláuber Beginnen gefeben babe er gebeten/ifim ju vergónnen nost) gu guter lefste ein Stuictein auff feiner Entber zu fpielen/ wol wiffeno / mas dee Delphin Natur: welches ifm vergoins net worben / Da baben fith etliche Dels phine sum Scbiffe genabet / uno einer ben aupgeworffenen Spirlmann auff Den SRucten genommen / uno lebenoig anś \&ano gebracht. Ziel anbere bets wunberliche Dinge mefor werden won biefem fobonen leutfeligem fifche ges fórieber. Darbon Ean man ferner le: fen Ariftot. lib + 5. c. 3. Aldrov.lib.I. de Cetis cap.7. Elianus lib.6. cap. 5. Fonberlich wie er bie Sinaben liebet/ ift in Gellii Noctib. Atticis lib.7+ cap.8. sulefen.

Num. 3. Ift ein Sopff von einem Secthiere/ fo fie Rosmarus 2 Ballrop/ oder See Pfero nennen/in Siróffe eines Џferber/ wie es auch Das 2nfehen bes Sopfes uno membri virilis, fo bars bey / su ertennen gibt. Das membrum virile, weld)es गुotoficher ino:

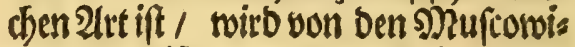
tern pulberifitet / uno zu Pluptreibung DeB Calculi ober Steins gebraudbet.

Num.4. Ift boll 2infehen ein graws famer Fif tiern Ser?suffel/ fonft Rana pifca. (5ii 
trix, ein Fifoct:frof genant wirb/

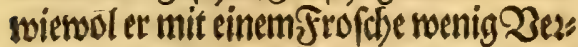
gleidjung fat. Roónte bequemer unter Die Siochen geteflet werben : iff 3 wo El: Fen uno Druber lang/auch bovo Ellen Dis cle nach ber circumferentz geredfnet/

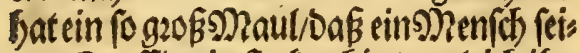
nen Siopf orein ftecten Eónte / gleic ibn audf) Oppianus befajretibet:

Eft turpis vifu molisfima corpore Rana

\section{Os aperit latum}

Sft faft nicht mefic als sopf und

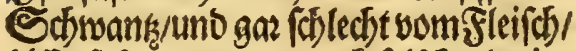
láfet fich / menn er nods frifd/alb eine Sinbes:?Zlafe ausbefnen / Daß man ein Sidft Darein balteno / als in einer $\{a s$ terne Durch fcheineno felsen Ean. Ert foll fonberliche sift gebraucher / Die Eleinen Fiche su betriegen und ou fangen / wie Alianus lib. 9+ c. 24. Plutarchus lib intr. animal, uab Ariftot. lib.9.c. 37. Ifrreiben. Er ffellet fich mit weit aufs gethanen Biachen smilcten Rlippen/ auch wol in Den Sdblict, uno betweget Sie bicken einfelm Scaare / bie er umb Den Nunb als einen $\mathfrak{B a r t ~ h a n g e n ~ h a t / ~}$ im 2 Saffer / wenn bie Eleinen Fif he Vauffenneife barnads lauffen/ in smzels: nung/ Speife su gewinnen / Dann thut er ben Siadfenzu/uno verfólinget fie als Ie/wie Oppianus d. I. faget:

Exitii ignaros pifces fic rana $\mathrm{ma}$. ligna

(hiatu.

Decipit imbelles magno deglulit
Er foll audf Denem/ po ba baden / fojaio: lich fallen; in Dem er ifre membrum virile ertappet/und barmit ju Sorunbe eilet/ Aldrovand. ex Gesnero lib.3. cap. 64. Dann bie Sippen fenno ibm rings berum voller fobarfer Záhne. Er foll auch bifineilen am Etranbe im Sirafe liegen. Uno fat man bes funben/Daß ein Fuchs/ fo ju Nachs am Etrande gangen/umb Speife zu fucjsen/ am Norgen von folchem fifiche mit Dem Beine if gefhalten worden. Jon. fton, cap. 9. de pic. 2 sir haben Ders felben Sildse stweene / Der eine iff bor 15. Sabren beym Siel gefangen worben.

Num.5.\& 6. Seyno Siachen bon groffen ungebeuten gtaufamen 20 alls oder Meerthunden / meldbe Salvianus und Euftatius unter Die 2 SBallifiche rectnen/aber Aldrovandus nidjt zuges

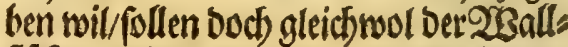
fif́che gróffe baben. Rondeletius fibreibet / Dak et einen mittelmeffinger Brtófe gefefen/Der 1000. Y) funb genoos gen. Ulno Gillius gebencét / Dap bie

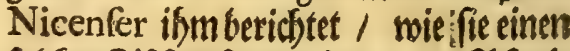
foldsenfirts gefangen/Der 4000. W Fund gervogen / und in ifm einen gankęen Menfoben gefunden/ wie auds Die Mafs fitlienfer einen gefangen/ ber einen gebars nifotten Mrann in fich gefdslucket ges babt. Uno meunet Rondeletius, oaf

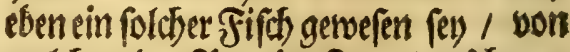
welchem ber Prophet Gonas er fohnaps pet/ uno burch ponderticbe Scjictung 


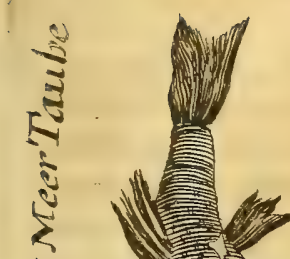

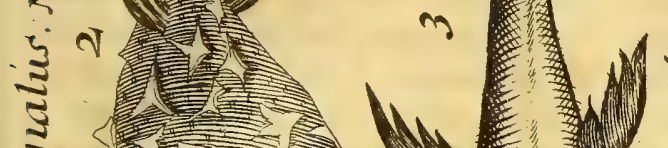

究

201

- rus $^{2}$ ) He (i)

\section{亲}
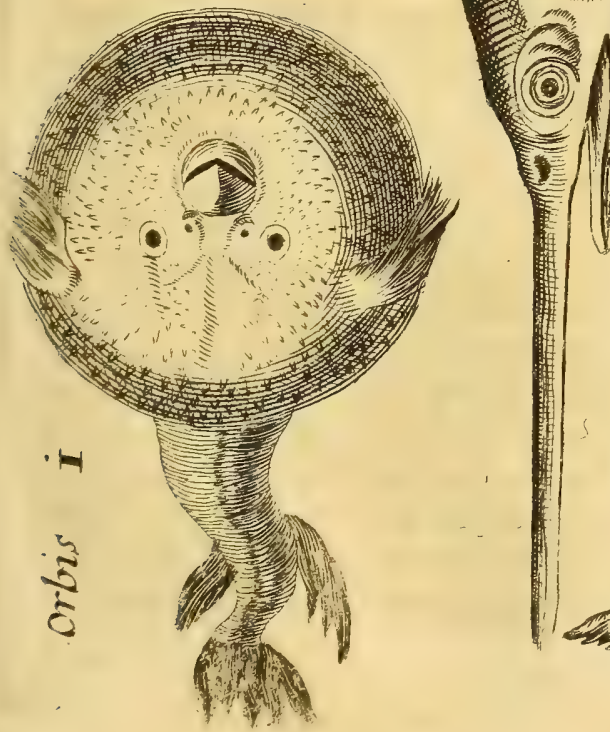

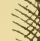
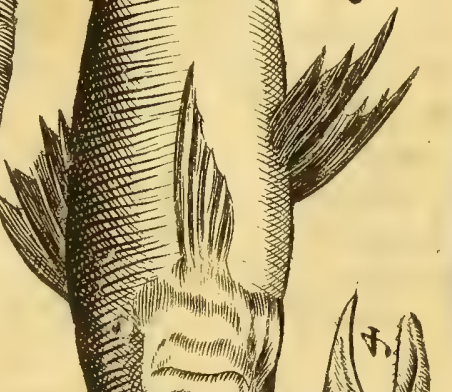
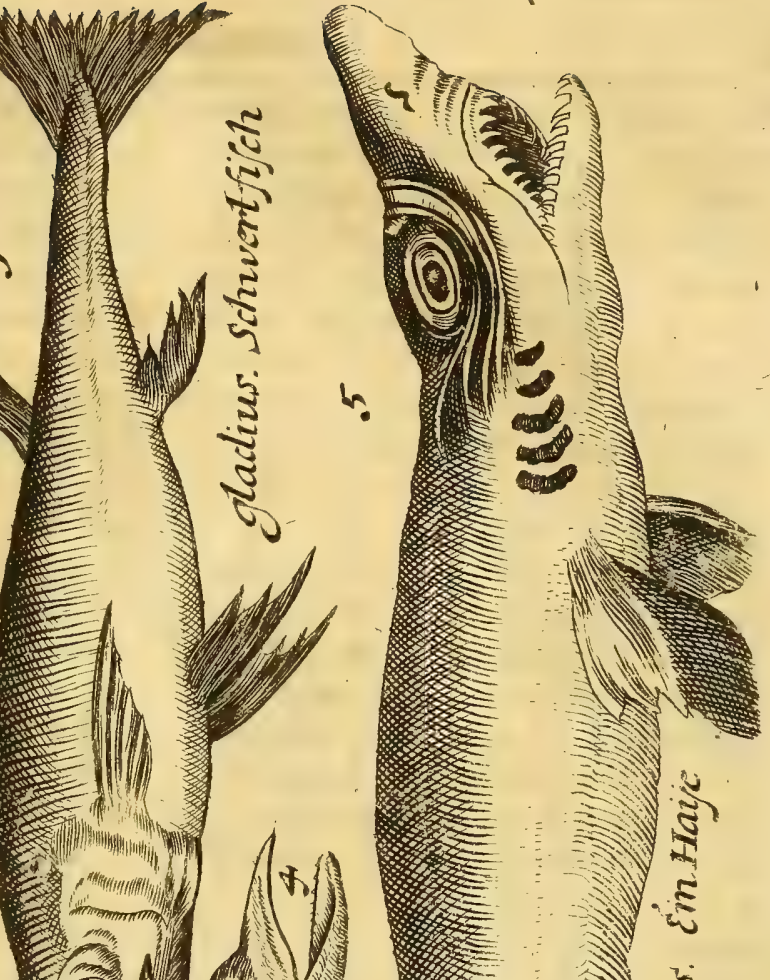


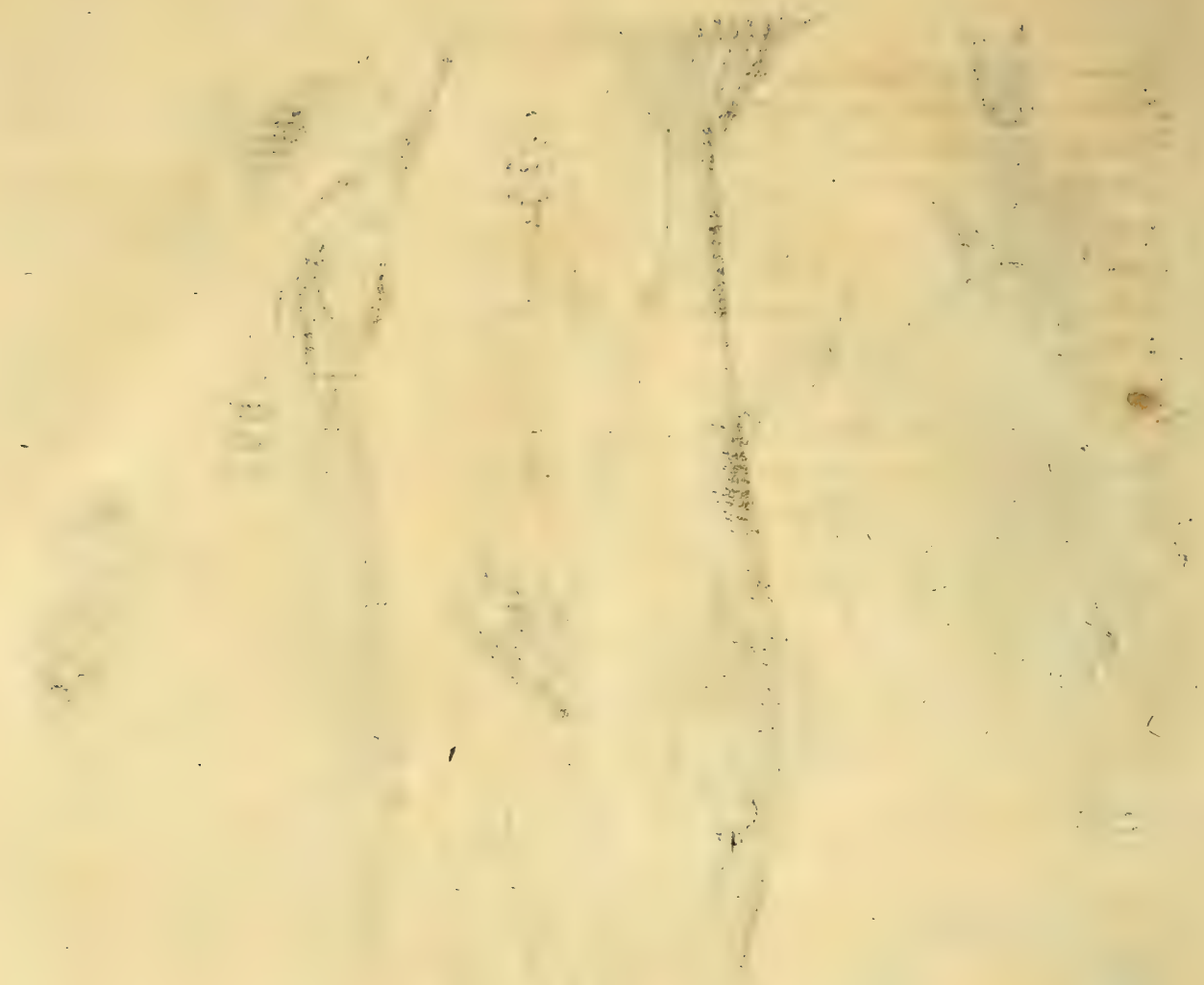


BSDttes bren Sage unverfefret Darein gehalten worden. Diefer obgefakgete Эias d)en hat funff und pechs fadse binter ein anver gefergte ftarcée fpisige Zabne/(mie Die gloffo-petres, und aud von etlichen Darfur wollen angefehen werben) Daher er vom Theophrafto Erefio Canis Carcharias genanot twito. Ein folcher Siff) ift in Mandelsilo Sitsmbianis firen Stbiffaftert gefangen morden/ Das von er aud 3 ericht thut pag. I 49. Das auch folche gefáhrliche Shiere bey Dem Charybdi fich auffigalten follen / melbet bie Shiftoria von einem Urinatore beym Athan. Kirchero in mnndo fubt. Daven unten Tab.26. foll get bactet werben.

\section{TABULA XXIY.}

Num. I. Ein Fif / wirb Orbis ges nunt/ iff gans runo wie eine Boptugel/ unv fo grof als ein Mzenfchen: Ropffi Eonte entweder nur Sopff un Schwant/ ober ß3auds und Schwank obne Sopf genant werben / tweil er nur ein Elein Saul und 2lugen an ber SBruft fteben bat / baher ifn auch etliche pifcem ven. tricofum nennen. Die Saaut ift ofne Scjaupen gan's rauds und fbarff. Er mirb im Ægyptifhen Meer am Ein fuß̧ bes Nili gefangen / uno weil er zur Epeifenidgt bienlid//wirb bon ben Ein roobnern bie Scaut abgegogen / augiges fropffet/uno jum Sierath in die șäufer auffgebainget. $28 \mathrm{senn}$ er an einemD t: te freey am Saben banget / seiget er mit Dem Mrunbe an / wober Der 2rsino fommt/ wie Rondolet: und Aldrovandus marbaffitig fotretiben / uno iff gleid) als ein 2setterbabn auff Dem Shaure. Dißs serebiefyet aber Durch) J̧ullffe Des Schwanthes/ber am runben Corpore allesefit bem 2 sinbe nachs lóuffit.

Num. 2. Ein rumber Tifa / wiro Orbis Echinatus gennnt / meil erúber unb liber boller 佸arffer Spişen mie

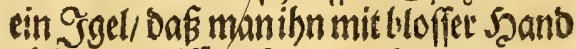

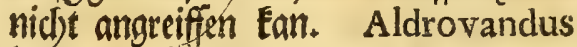
befforeibet ifn lib.4, cap, Is, de pircib. ausfubrlicher.

Num.3 Sft Gladius ein Sdhnetoto Fiffo vier Ellen lang/ felliger ift vor 12 . Sabren bey 2ipentase sefangen noor: Den/ bat gar eine Duinne şaut / Dap if Die Eleinen Fiffore gerne answacten/ Dar: um hat ibn Die Natur Das Sabroetdo zur 23 ebre gegeben / fol fids für bem WBallfifo fehr fürchten / uno ifn meis Den/Da er boch/swenn er feirte Stircle suifte/ ( wie Aldrov. faget) Eónte Dent 23allffipte fonablich renn. Er foul bas Edfwerbt hey anberthalb Sçand breit ins Schiff jagen fơnnem. 2 Sirt bes ferrieben von Eliano lib.3. c.4. Plin. lib.32. cap . II. Aldrov, lib.3. c 20.

Num. 5. Sft auch eine Int bon Den obgebadfen Carchariis. Diefer if gar jung uno nur wier Eillen lang / fô E iij 
len fünftmabl fo grof uno Den $23 a$ lls fifoben falt gleid lenn. Dif ift cinter

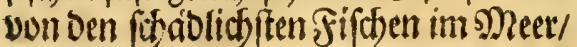

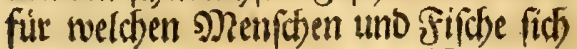
fürd)ten. Er wiro vom Ariftot, uno Oppiano Galeus \& Muftelus, bon Den Plortugiefen Tubaron, von Den Scllänoern Şayen / Şund:Fifh ges nant. Jm 4. S beile Eap.4. Der D/ts gno. @dhiffabrt Hugonis à \&ino/chots ten wirb erzeblet / Dap bey oer Stadt Cochin ein foldfer Fif cinem Sobts: mann/ber umb Das Robe finten am Schiffeinsubengen / fich am Seil ins SGaffer gelaffen / ein Bein abgebifen/ uno als er Darnadh greiffen wollen/audh Den Arm und ein Stuck aus Dem Şins tern mit weggefdhnappet/ ungeadhtet Die anbern Bothsleute ftarce auff ith zus gefthlagen. Mandelslo be fdreibet eis nen foldhen Jifw/ Den fie unfermoon bet Imfel Zeilan gefangen/gat ausfúbrlich im andern 2 ud Eap. 13, Dergleichen Exempel / eben

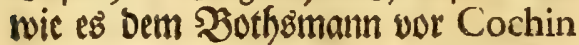
ergangen/ ersefblet auch George 2Inder: ren in feiner So biffabrt/fo er aแb Jndien nadb Dem Sothen Meer gethan. Es faffen/ forreibet er / in unferm Botbe etlicíe unfer 2 zólcter uno fifcheten / uno als einer unter ifnen auff Dem 2000 ort faß / uno bas sine SEein ins DGaffer bieng / Fommt cin Hay uno beiffet ifm gefurwinde bas Sjein ab/ ja er fätte ibn gankz weg getriegt / wenn nidyt bie an
Dern ifm wairen ริ รุulff getommen. Da bis alfo paffiret way lieflen es unfes re Vólifer balo aub ber ache / uns prungen zwey) Sage bernach etliche in Das Meer/ umb fid bev ftillem 2 setter ju baben / fie waren aber Eaum inoas Warfier getommen / murden jtoeene von ibnen ourd) foldse Hayen unter Das WBaffer gejogen / uno famen nidbet wies Der empor. Die andern eileten mit Sdjrecken wieder zu Schiffe. Dar: aufferging von unferm Schiff: Patron cin Defefl Das Eeiner fich folte geluften laffen/im Meer gu baben bey verlufteis neE Sabrę Sold.

\section{TABULA XXV.}

Num. 1. Diefer fremboe Til wirô Serra aud) Priftis oom (d)nei Den ben Ramen belommen/ weil er wie eine Erige fonneibet im fónell lauffert. - Aldrov nimpt Die Sefdrteibung Deffel ben aus Dem Clufio lib.6. c. 19. Man findet fie gros und Elein / wie wir Dann Der Edynabel in unter fhieolicyerOirós fe viel baben / Die Girofien faft sneen El: len lang / und oben / Dafie am Siopfie gefejen/eine balbe Elle breit. Wonber Eleinen 2trt/ Da Der Schnabel noch am Jif the fiket/ haben wir jweene. SEefin ben fich gemeiniglich in ber 23 efts Sie.

Num.2. Remora ein Jiffh / Deree wir greene baben/wiro bon unter/chies. tioten 


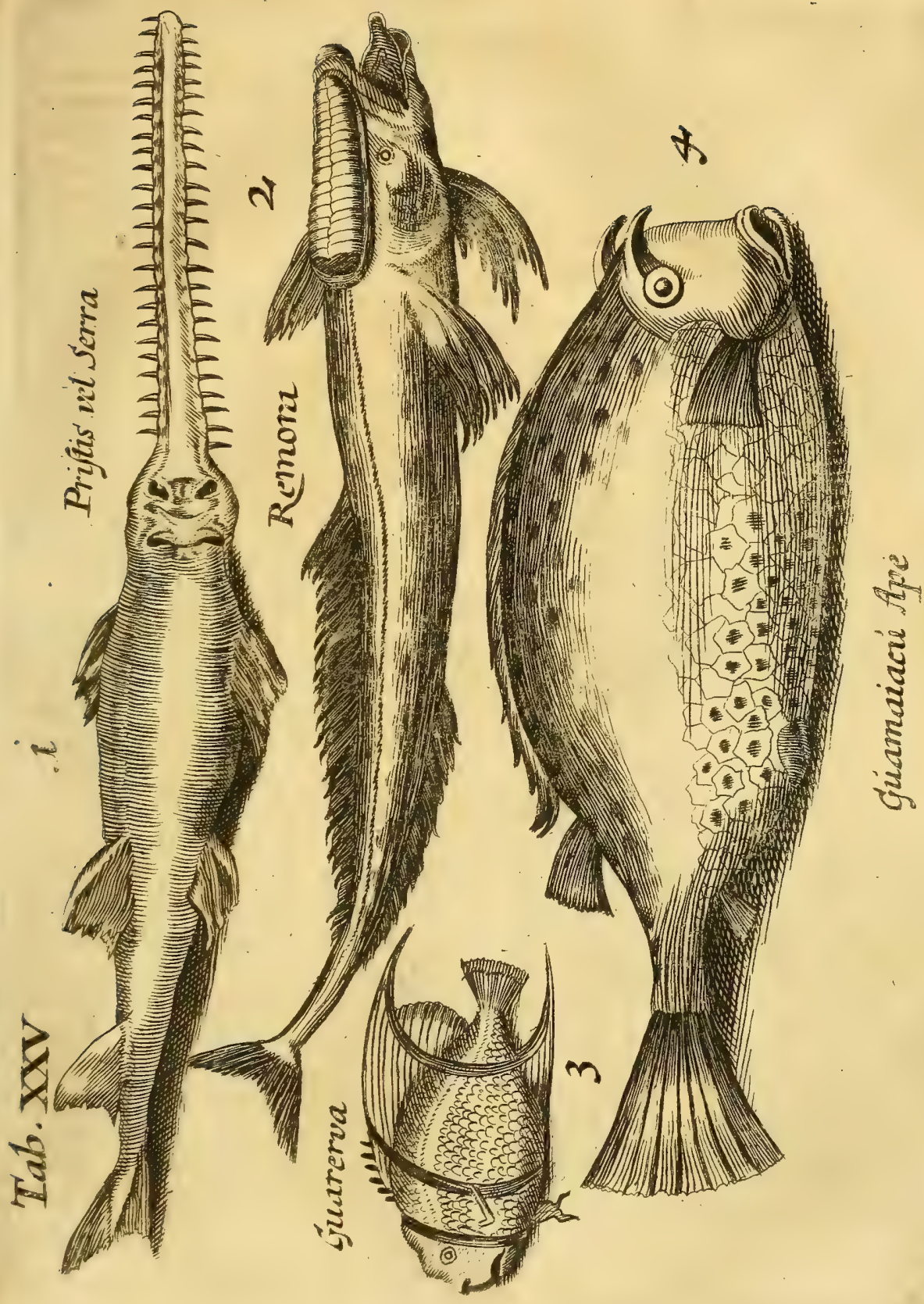



lichen fonberlich vom Plinio 1.32. c. It Der ifn Echeneis nemet / wie auch bon Aldrov.1.3.C.22, befdyrieben/und ift ein 28 under Der Natur/ Daß ein fo Eleiner

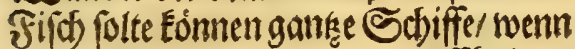
er fich unten Daran jauget / auff falten. Kircherus will gwar lib. 3. part. VI. de arte magn C.I.S.3. Diefes für $\mathfrak{F} \mathfrak{a}$ belmercế halten/ uno mernet / es lónte nicht fenn/ Daf ein fdgrwerer Corpus von einem leidfern Eónte berweget uno ges balten werben/gleich am Magneten f Feben/wenn er gegen einem ftucte Eifen Das/ftwerer als Der Magnet in freye Euft gehangen wito / fo Ean Der Mings net Daş Eifen nicht anficis jieffen. Scaliger aber/Der Exerc.218.5.7.\& 8. Die Siraffi Der Remoræe aud glàubet / wil ess niddt Den beÉanoten naturlicten Ut: fachen/fonbern Den occultis qualitatibus zufchreiben. Lund geftebet Kircherus d.1.p.523. felbft $/ \mathrm{Daf}$ animalia aquatica occultis \& majoribus viribus predita fint'quam terreftria.

Plinius und Aldrovandus aber bringen glaubwoirdige Exrempel herbey/ Dáp Die Schiffe von Der Remora feyno auffigehalten worden. Dis hatte Antonius in ber Seefhladbt bey Actium mit Schaden erfabren / inbem fein Sutiffoom felben Sifde fitlle fithend waire gehalten rorden/Damit Sainfer 2Augufus ifm hoitte Den Yortheil ab: lauffen Eơnnen. Dergleichen Sermmung Des Scjiffes bat fich auch beym Cai
Caligulx @chiffahrt begeben. Dar: bon Sveton.in vita ipfius. Aldrovandus faget/ Damit man nicjt bencfen folte/Die Alten bätten uns biermit etwas Unmaarloaffitiges binterlaffen / fúfret er Dab Zetrgui enfis Equitis de Cardinalib.p.99.00 Francisco Cardinali Turonenfi mit eim/velibem fein Sthiff/ mit welchem er einft aus Franctereid in Ttalien fahs ren wodlen / aud Durd) Diefen Fif Echeneis ober Remora mitten im

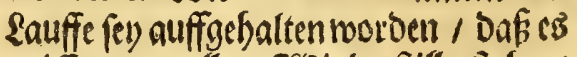
múffen yor vollem 2 sinbe fitlle feehen/ Dergleichen erzefletScaliger dicto loco, Dapabiel Schiffe mit eimander eine Jielife gethan / unter Denen ift Das eine alleine unberweglich geftanoen / und sie andern fenno unverbindert barvon gefrgelt. 2seldbes ber fiffol Remora auffge balten.

Sd) will noch ein Exempel einfíbren

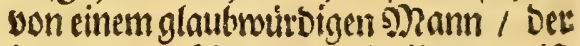
in anbern feinen Befchreibungen ift ridytig erfunben morben. Hugo Sinds fogot ferget in feiner Oriental. Schiffe fabtt c. 48. 2Alts mir aus \$yortugal nad) Mofambique fegeltem/ und unfern (Sours nad) einer geraben Sinie zu nab: men/ und giengen bor: 23 inde mit yols lem Segel / und inbem mir 14. Tage einen Cours gefbalten / befunoen wir Durch) Obfervirumg Der Graduum, Daf roir nidst alleinnicbt fortfamen/fondern in etropas zurúcfe gemichen / mit aller Ber: 
Wernunberung / weil wir gut 2 better uno 2 Bino battem / uno Die Erfabrung uns auch gelefret/Daß am fellen Drte fein miderwertiger Strom zu vermus then/Der bie Fabut verfindern móchte. 2Sirftunden alle befturket / wouften nicht mie bas sugieng/ stliche menneten/ es mare Zauberen. Shngefefye fihet Der Steurmann binten am Schiffé hin unter / uno wiro eines groffen breiten Sifts: Sckwankges getwabre / weldjer fich batte am Sointertbeile des Sdsiffes angeleget / Der \&eib war unter berm Edhiffe/ und ber Ropffam Bioht fefte/ als biefer Fifch Durch groffe Mnube Der Sotholeute mit Saneen uno Stangen lop gerifen/ gieng bas Sdbif wieber genuinfchet fort. Dín einige Exentpel tointe Kircheri Mzennung wiberlegen/ und erfhalten/ oa 2lten von ber Remora gefdrieben/ wie er Die Sdbiffe auffbalten tơnte. Do man fhon Deffen nicht naturtliche Uriar

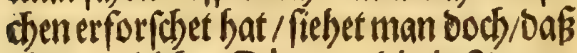
viel Dergleidsen Dinge / Die in 2lugens fdein tommel1 / Den Werftand vorbery gehen. Daf meines Eradteng Chxremonianus Trallianus nictet fo unges reimet gefaget : 2lls allerthand 2 rit Sifthe auffige perset worben / und unter Denen auch bie Remora ( weldchen bie (Bitiechen Exchineiden nennen) Diefen ( nemlidf / Dergleidsen Sifd) babe idf geperen/als ids im Sicilifichen Mreere

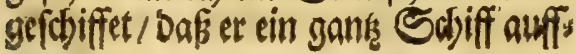

gehalten / bif fo lange ber Steurmann ithn vom Sdiffe reggenommen; unb Die Seynefenoe bartiber gelachet / als wenn er eine Fabel erzeflete. 2 23orbels auch in etlichen naturlichen Sachen ber Antipathia gedacht rwurben. D ibr lies ben Seute/ biel Dinge gefcheben/ bie wir vor 2tugen fefben / und boch wol ifree nas turlliche Mr facben baben I ob wir fie fchon nidft wiffen. Soldbe nidft ers forfoben wodlew/ if swar nictst fo unbils

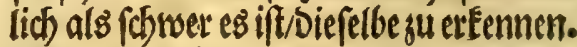
Plutarchus lib.2. Sympof.pag.64I.

Somft will Kircherus naturlidje Ulefacten geben / warumb Antonii Schiff nicht fort gelont/ weil eš nemlich in ber See bifineilen contrarie Stros me gibt/ po das Sdjiff auffbalten tón nen. Plutarchus aber gibt viel getwifa fere Urfachen / wenn man bie Remoram nicht anféfen mill / nemlich : Daßj Des Antonii Schiffich mer/ unberende und mefye zur গJjeacht alb zum Streit und auds mit unerfaftnen uno ungeuibs tem Volcte aufigeruftet und berfefen Des Cafaris aber leidst und befende ges weren/mit weldsem er ben Antonium umblgehen fónnen. Plutarch, in vita Antonii.

Num 3. Dieper fifich Guaperua won den 2 tafilianern / roofellof er ges fangen wirt/ yon Den Sortugiefen aber Peixe Porco genant / beift fo viel als cin 23 ilb Sdjwein/wito bom Jonfto. nio lib. 4, de Pifcip.cap.10. aus Dem Marck- 


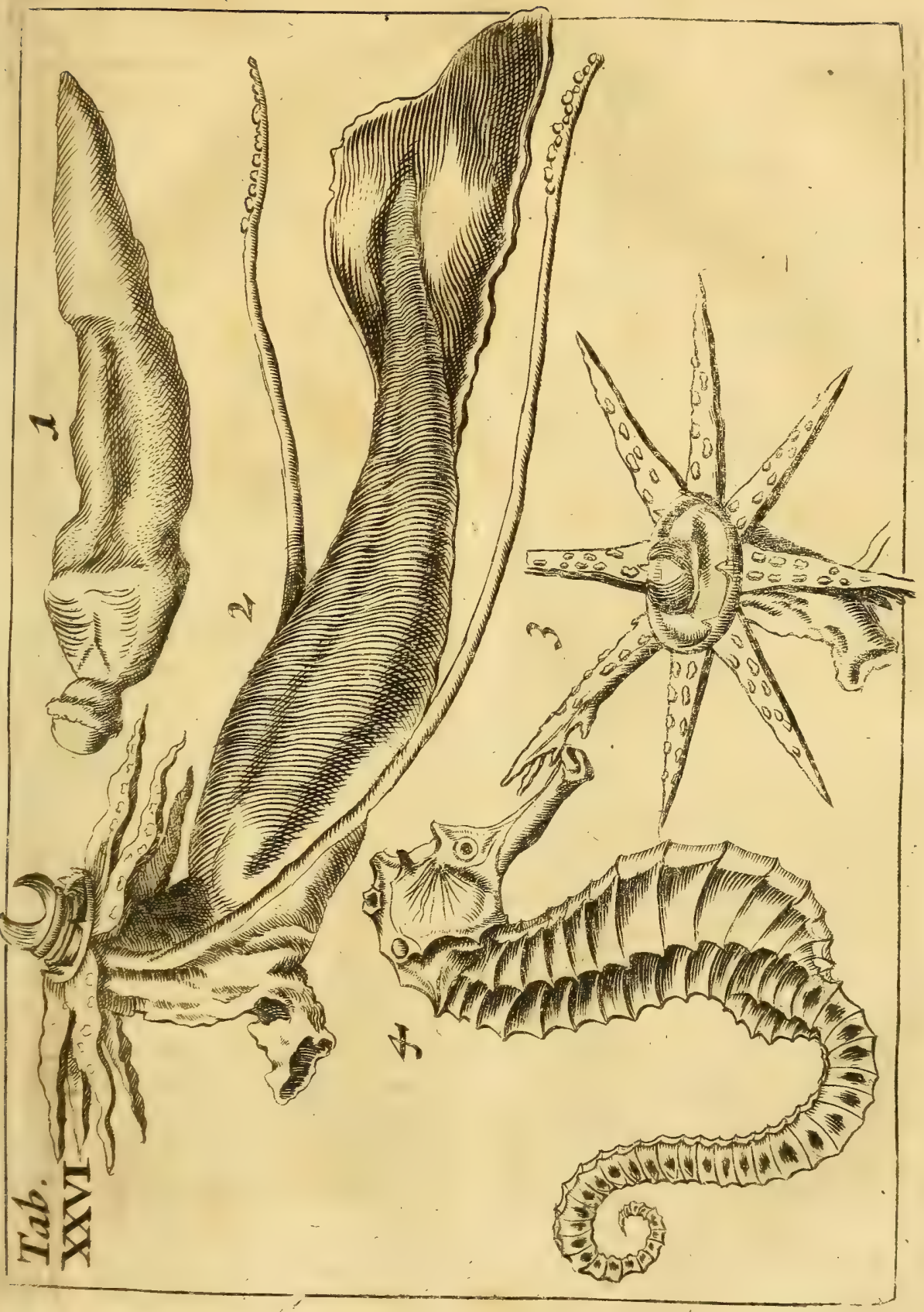



Marckgravio lib. 4, de Pifc.c. I 2, aub: fubrlich be fdrieben / weldser faget / Dafa er nidf gu Eodjen/ fondern nur ju braten Diene.

Num.4. Ein Stafilianifher Fiffor bey ifnen Guamajacuape fonft Drey: anglictster Fifo, genant/weil et auff bem. Jెaudbe gants plat wie aud beiben Set: ten/melche auf Dem Sicten fdjarff jus fammengeben/ und alfo oie Forme eis nes Sriangets machen/baben eine barte fobarfie Saut ofne Edbupen/weldhe boller funffeckte Figuren ît/bey) etlidjen feono es nut blofie funffecte $/$ bey etlis dren aber fínder man in Den Pentagonalen Sterne. F: fesno zmenerley 2rt/ efliche baben úber Den 2lugen Şót: ner/ etliche feund ofne Syorner / wit bas ben bon beyoerley Alt etliche: und auds einen/ weldser auffoem Biucken fo breit als am 3audje/ uno baber ein Qua: Drat Fif ch Eonte genant werben. Sie follen gar ungefuno uno faft vergiffet feyn. Sie werben vom Aldrovando lib. 4. cap. ultimo und Georgio Marckgravio lib.4. de Pifcib, Brafilian, cap.I, befdyrieben.

\section{TABULA XXVI.}

Num. I. Iff ein Fiff Monachus Marinus genant/ weil er am Sbertheile faft einem SMund) gleich fiebet / foll zu Enceflyuren lebenoig gefangen worben fein batmeder Sduppen noch Dinnen ober floffebern. Aldrovandus bes fchreibet auch einen Monachum lib. 5. c. I 7.bat aber Schuppen/uno fiebet un= ferm nichtebnlich.

Num,2. Stt eine felgrame art eines

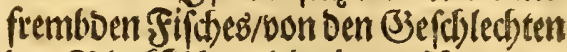
Der Blactefifbe / Die einen fobwarken humorem, twie Blact oder sinte bey fich babeniderer Dreverley) 2(rten ergeh: let werben/ als Da feuno Palipus, Sepia und Loligo, uno werben vom Ariftotele hift 4. c.I. Plinio lib.9. cap. 29. Matthiolo in lib.2. Diofcor.cap.20.' A.ldrovando lib. de mollib, uno ans Dern mebr eben auff foldye Art als lestit balb folgen foll/beforieben. Der Un ferige ift unter bie Loligines zurechen/ nodj jung uno nur einen Fus lang / fols len fonft in shannes Biroffe / und etlicbe viel groffer erwadbfen. Seiner ber Autoren aber bat ben Siopf und Sdyna bel fo eigentlich algefbilbet / als in unfer Figur (po Dem Şollänbifchen in allem efnlidy/ nur daf unferm bie langen jwo Jinnen abgebrodben) ;u feben. (Ë fors lenfich alle brey 2 reten in ber Spani: foben uno Stalienifoben See am mei ften befinven. Uno weil felbige Airt Fifche in Seutfiland nicht viel gefes ben/bor etlidgen wenig Jabren aber eis ner in Scollano / und ciner in Scolfein bev Scamburg gefangen root Den / fo fur erforrecfliche Meertounder baben wols lengebaltenwerben / eradjte idjs nidjt unbequem gu feyn / felbiger gefangenen $\mathfrak{f}$

fis 
Fifche Sefichreibunge mit einjuführen/ uno etwas ausfúbrliçer Darbon zu bes ribten. Des Şollainoers lautet alfo:

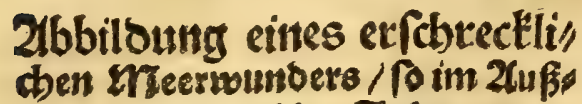

gang bes 1661. Jabre in zollano gefangen norben.

NB.

Diefes s)eernunder int in Solland grwifden Scheblingen uno Satwig auff Der See/ an Dem Drt Da Die Engs lifde Schiffe lagen/ Die Jhre Mrajeftát bon Sirof: Szritanmien abfoleten / ge" fangen worben. Uno bat nad) bem er gefangen gervefen / nocb orev) Stunoen gelebet: fich aber in fangen fo nounder" graufam angeftellet / Dafibie Fif cher ges meynet / Der కeuffel wáre felblt im Nes ne/ aud fich nicht ebe ergeben / bie man

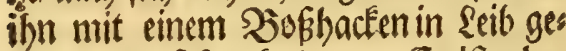
hauen/und feft gehalten. Eve ift obns gefebr orey uno cinen ballen Fus lang/ bat ein gal wounberliches Scaupt I uno auff Dem scaupte einen actitfantichten Stern/ bey nabe eines Fuffes lang/ Dar: bon zroeene mit Saut úbersogen / wie bie જllugel einer F̂ledermaußs. 2lufi Dem Stern fethen bin uno wieder gar viel Snoffigen mit einer Eleinen Erone umb. faffet/ uno wie Der Fifos nod gelebet//o baben Die Sinopffigen als Eleine Spies gelgefdimmert. 24ธ Dem Stern ge:
Get ein 20 (ber Sd)mabel/ ( po bom Scaliger exerc. 218. bequemer ein Slapas gojen Sdinabel genennet wirb) weldher Ean auffund sugethan werben. Unten bat er énen Siúffel oder Mlund als ein Schroein/unb eine 3unge Darein. Bmo fchen Dem Siuffel und Dem Stern ftes ben Die Alugen/welche beym \&eben Des Fif des fo forreclich anjufeben gewe fen/Dapis man für Dem 2 inblice fich ento Pegren múfien. STacboem nun die 2 tw gen ausgenommen / umb gebalfamiret fu werden/fbat fichs befunden / Dap ber innerliche Siern im 2ugapffel Die. (Jie: ftalt und allehen einer Jerle gebabt. Die Augen an fich felloft hatten bie grós fe eines groffen Salb 2üues / und iff Dem Fififer vor bas eine 2luge bunbert Bjulben Scolloindife) geboten worben. Unter Dem Stern oder Crone/fo es auf Dem Siopffe batte/geben aus zweene lans ge 2(rme / olngefehl ztwev Finger breit. Er bat 2 Derter / Daourch er Epeife zu fich nimpt/ uno bat Feinen 2 lusgang. Man batauch in Deffen seibe fein (S) Dárme / fondern lauter seber und Fett gefunden. Daber bie Phyfici uno Bjelefrten / Die weit und breit gereifet/ beEennen / Dergleichen an Eeinem Srte/ meder in Stalien / Turcfeyen ober Jin Dien gefunden zu haben. Şalten $\mathrm{eb}$ fur ein fonberlich 2 Bunber: Bieforopfiel Deffen SEedeutung Dem allerbódffen betandt. So weit Der Scollains Der. Aber Die Phyfaci batten Den Aldro- 


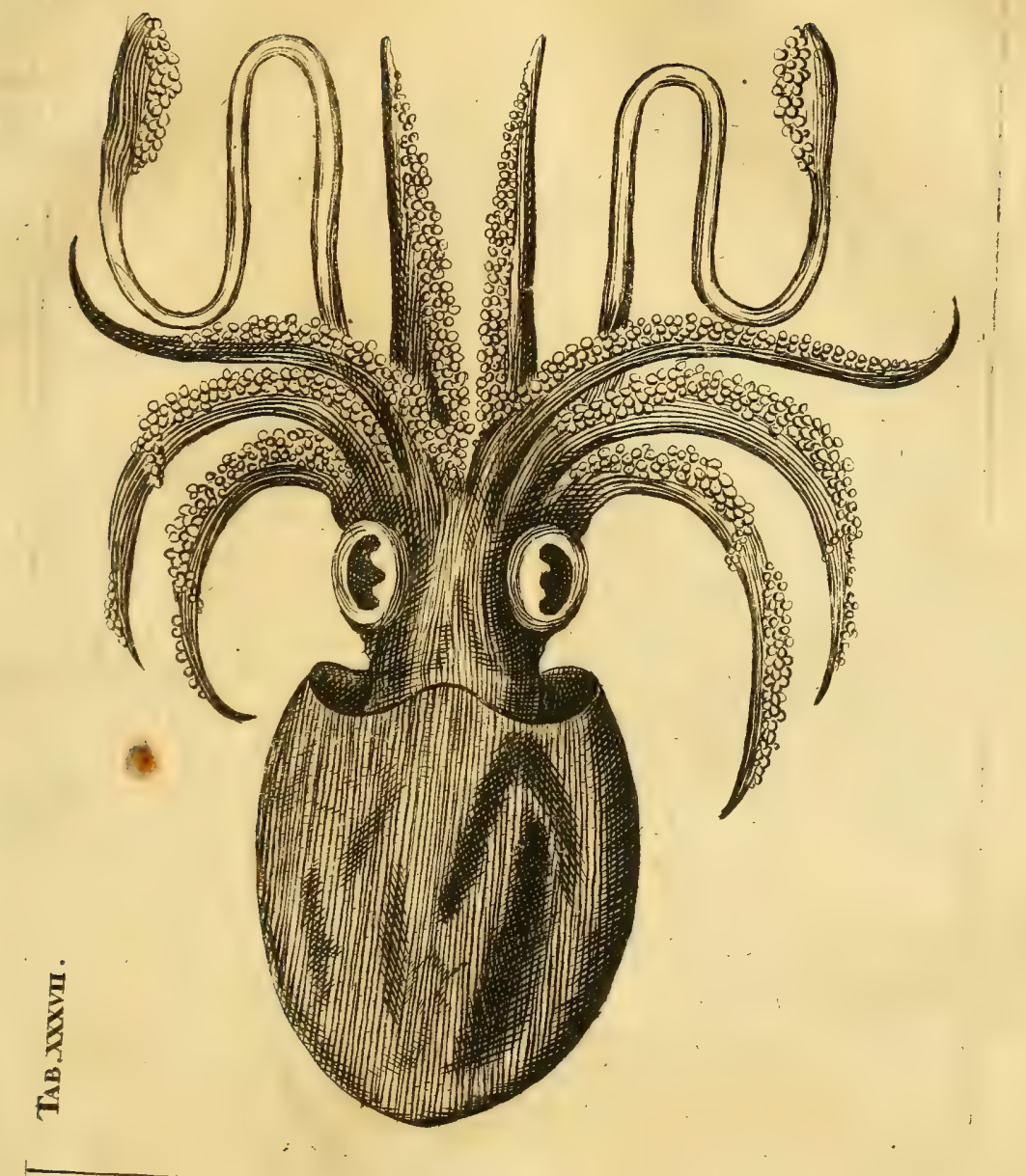



Aldrovandum ober jonftonium lefen follen.

Folget ber Şamburger Fild .

Jie warbafftige 2 erarety bung bes wunberfelzamen Sis fabes / welcher am 8. Cage nach Der Zimmelfabrt Cbrifti $16 G_{2}$. in ser 促lbe unfern ber Stadt 万amburg ift gefangen worden.

Sat bas zuirchen Scheolingen und (Eatroid) jum Enve Des verwichenen 1661. Jabres gefangene 2)Rer:203un Der ben Den Neugierigen viel 2 Bunders erzeget / fo wiro Diefer allhier gefangene Fifoh nidyt meniger thun / Dann er an sumberbafret 2irt Dem andern meft vor:alè nacbgefet. Seine sainge mit feinen Winnen oder Floffedern ift Drel) Juf lang/Das sorpus an fich felbit ift oval-langlich / uno bon anderthalten Fup: Die STreite aber Deffelben Drey Duart eines Futfer. Die jtoo Din nen som Siopffe bin / fino gleich stwo Zabacts: Wfeiffen/ weif uno glingerno/ Derer Enoe aber vergleichen fich mit AD: lers:s Slauen / bejeşt mit einer weiffen

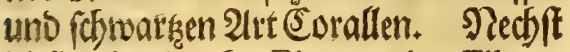
biefen ferno acht Pinnæe oder Fibræ, roic ein groffer Stern aufgebreitet/roels che alle sleich fam marmoriret fehen/ und init (Sorallen gegieret fino. Sein Sg?uno/in ber 9litte Diefes Stern / bat eines balben Sbalers (Strófe/ noraus

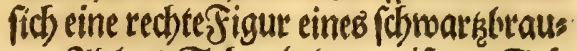
nen AlDlerв: Schnabels erweifet. Sei ne 2lugen find in ber Siunde als ein paar Sd) en= 2lugen / mit fdjroar'sen 2lug:21pffeln. Die Saut uber feinen Sividen if rectst fobón matmotiret und weich. Der Siucfgrad aber ift fo bart als sine Sdjilopatte/Dap fie/mann man Darauffelopffet als ein barter Sinodisen

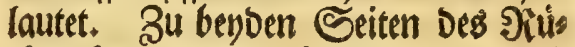
cfens bat er zwey levige Yafdyen / wels che man wol 2 Binthafden nennen mócte. Er ift ofne Sofrwans. Ulmb: geEefbret fiebet man nod einen \$runo/ aber gankbohl/foredht unter dem STopff ift. Sein 2 aud ift eine lebige $\tau_{a}$ fdje/ Dann er ofne (S) Daime / ift fonften bon unten langft bin gans weif als eine. Edbolle. Und ift biefe Figur nad) ifo rem 21 briffe/wie er auffoem Jiucten an: gufehen/abjonoerlich mit beyfüget wors Den.

YSer fiebet nun aus obgedachten Zejchreibungen uno Figuren nicbt/ Da alle oret) Fifche falt einerley Art fentlo. Nur Dafi ber Sollánoifote uns unfer lainglicter/ Der Samburger aber etwas runder/ woic Dann benderley) 2Art bey Den Autoren zu finden/ und alle unter bie. Mollia ju rechnen. Dann Ariftot. lib.r. hift. c.4. \& 1.4. c I. madyet 4.genera animalium exangvium; molle; eruftatum teftatum \& infectun. Die mollia doer weicben ( fo bon Den $\mathcal{F}$ if 
seut wie Aldrov. de moll. p. 9. (d́reibet) merben barumb alfo genant quod osfibus \& vifceribus careant, bafi fie weber Snodben nod Dairmen in fich baben/und werben Derfelben Dreberlen Arten gefeket/ als Polypus, Sepia uno Loligo, welldse Dem eufferlichen 2 nfes ben nach / man einerley fobáken foltel mie fie aud faft einerley Qualitáten feyno / bennody nach Ariftotelis $\mathfrak{V}_{\mathrm{e}}=$ richt/ in etwas unterfchieden : Daßnem: lich/ ob fie stront alle 8. Fúffe (meldse bie obgebachte Autores Sternen nennen) baben I aber felbige nidjt auffeinerley soeife gebrauchen tónnen. Det Polypus ( welcher zmar Den Namen bonviel Fuifien hat/ aber tefte Scal_exerc. 218 . c.2. ipfi pedes vix hac appellatione digni) hat lange Sbeine uno fursen Eeib / und Ean friechen (nad) 2Irt Der Saupen) Sepia aber und Loligo ba: ben einen groffen \&eibund Eurge Sjeine/ uno Eónnen nur fobsimmen : Der Polypus, wenn er Unmitter vermercfet/ fauget fids) an Fel/en/Sepia uno Loligo aber fobsimmen und fpringen empor. Der Polypus foll audb Eeinen Fiuffel baben / gleid) wie Sepia und Loligo, auch nidjt fo cinen harten Siúcf́grab als Sepia. Sft alfo meines Erad)tens Der Saamburgif(t)e unter Die Sepias, Der Un, ferige uno Şollander aber unter Die Loligines zu redfnen.

S6 nunstwar Diefe 2att Silobe in uns fern. Ealten Noroifchen Eanoern unges mein / weil fiefich meift in Den warmen Mnittrigliden Seen auffibalten / uno bifweilem obngefefreiner / fo entweder Durch continuirlicten Sturm Does Durd) 2 Infaugung an ein Schiff in biefe Serter gebracht werben / po penno eb Ded Defrwegen Eeine erforrectliche Meerwumber / Dergleichen Die Naatur zubor nicht orbentlicher meife eingefuifso ret. (S) leichnol aber wenn man Die Sciftorien / fo von Diefen Neertfieren auffigezeichnet fino / beobad)ten will / fo fónnen fie 23 unders gnug madjer. 2Bill bem \&efer \&uft balber einer uno anber Sciftoric oom Polypo, weil fie faft einerley) (Se) follechte / und wie Plinius lib. 9. cap.30. (d) reibet/ zu einerley Brioffe erwadben fónnen / mit eins fübren.

Athenæus lib.13.c.6. faget/Dapioie Polypi Durd langwierige Zeit faft in

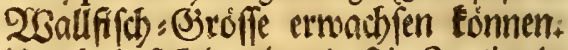
un no habe fich) legeben/ oa in in Stalien in Der Stabt Potfiolo, foam Meerftrans De gelegen / ein ungebeurer Polypus Durch Die Scóle ober Stsluno / Durch melchen Der Unflat Der Stabt in Die See gelauffen / fich in ein Şaule eines Fif hbindelers eingefthlichen / und eine groffe Tonne mit eingefalkenen Fifchen zerbrocben / uno das sefte beraus ges freffen. Der Sauffimann zuerfabren/ neer ibm folchen Schaben sugefuiget/ freflet einen 2 sàdster an felbigen Drt? 
mo ber Sonnen mefr ge/tanden. Zur Nachtseit Eommt Der Polypus, einum ungebeur groß 3 S bier / mieder / umbgibt mit feinen 2 trmen ober 3 einen eine fri: fibe Sonne/ Dructet fie fo bart / Daf fie enghet) fpringen uno Den Jifo bergeben mus / und alfo / nach Dem er eine gute 2) Ralseit gehalten / fich Durd Den Ein: gang roiederumb barvon in bie See madtet. Dee 2 Sádter ob folds un getwo hnt Spectactel erichrocten / bleibt für Angft ganf ftitle / láffet alles gefdses ben / uno erjeblet frube Den Jerlauff. Do Diefe Gefchidlte Dem Siaffimann stwat etwas ungláublich borfompt/ uno Dody Den neuen Schaben wieber fibet/ gehet felbut mit etlichen getwapneten Mánnern unD fidarffen Snitrumenten/ felbigen ungebetenen (ja)t zu empfan: gen. Uno als Der Polypus burd) fei: nen geroobnlichen (3)ang fich wieber eins fiellet/ wird ifm Das sod verrennet / fei ne SEeine alogehauen / uno alfo getób tet. Deffen Corper Elianus, wie er fdret bet/ felbet gereben.

Ylinius ergeblet lib. 9. cap. 30. wo nicht eben biefelbige / Dod Dergleidsen Safforie mit etroas andern lumbjtan. Den/Denner faget: Dapfie in Spanien in Eartejafich babe zugetragen / uno fer Der Polypus mit Sounben gebeget nor Den / meldbe ben ftarcfen (Bieruci vom felben Sbiere nicht tool baben eroulden Eónnen. Utno fescet eine ungláubliche Broffe Deffelben Fifdreb. Woenn Dem
Sslauben juguftellen / ift nidbt zu vers nundern/Dap wie Elianus lib.7. C. II, (d)reibet/ ein Polypus einen 2loler ges fangen babe / uno im Meer ertraincfet. Dann als Der Fifd am Strande auff cincm Stein in ber Sonnen auff Dem Siucken gelegen/ uno bie ßieine bon fitd) geftrectet/ fey Der 2toler/ in Neynung einen Siaub ju erfobnappen/auffifnges fallen. Der Polypus aber babe feine Fúffe uiber ibn jufammen gefollagen/ uno alfo den 2 doler mit fidy ins SOSaffer gefchleppet und erfáuffet.

Dafi gar groffe uno gefábrlid)ePolypi fenno/hefráftiget audb bie Sciftoria von einem Urinatore ober $\Im a u c b e r / D e r$ un ter Dem 2 Baffer Die Perlen fuchet / aub relation Athanafii Kircheri in mundo fubterr. lib. 2. technico c.15.

(E) fen zur Zeit Dez Sónigs Friderici II, in Sicilien ( mup umbs Sabs Cbrifti 1200 . genefen (eyn) ein Urinator oder Werlenuno Corallen: Fifcher Namens Slicolaus gemefen / welcher von Gugeno auff fich jum 2 affer ges balten/ im fobroimmen uns untertau क्रen fich geúbet/ uno feine Nabrung mit Corallen uno Dertentfifoen gefuchet/ und in Das See?eben fich fo verliebet/ Dafer offt 4. 5. Sage in Der See geblie ben/uno fid von roben Jifoten erbals ten / uno feine Natur bon Der gemeinen sebens:2lrtaligenefinet / Daf er fo wol in als aub Dem 2 Baffer offt einen gan: ken Sag ofne 2lthem: hoblen leben tơns $\mathfrak{F}$.ii

neny 
nen / und alfo einem Amphibio effnlis cher als sinem Menichen gewein: bat

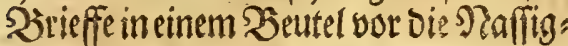
Eeit nos berwabret Durch die See bringen Esmen / Derwegen er audb vffic als ein Bote gebrauchet worben / uno haben ibn Die Balleen bifweilen in gropten Eturm angetroffen/und vermeinet / waike ein Neerwunder. Wun Denen aber/ bie iffnetEand baben / ifter ins Schiff genommen und gefpeifet roor: Den / uno nach ein wenig Sprachbals tung bat er fich trieder in bie See ges machet / uns ift Datwon gefdow men.

2lb einft Der Sionig in Sicilien su Mefiana fich auffielt unb viel ungláub: liche Dinge bon Diefem Plicolaus ge bóret batte/ befam er \&uft Diejen SJen. fochen ju feben/ weld her/mach Dem er ets etliche ₹age zu2Baffer uñ?ano gejuctbet unto gefunben worDen/fich bor Den Sios nig ftellen múffen. Uno alอ ber Sio: nig viel felçame Dinge/ fo im 2 Baffer befindlicb/von ifm vernam / wurbe er begierig/Die $\mathfrak{B e f} d$ affenbeit Des nabe ges legenen Charybdis ou erfafren. Es ift aber Charybdis ein gefábrlićer Drt in ber Seesnifichen Sicilien und Jta lien/meil bafelloti Das surrfeniforse uno Sicilifhe Sneet zulammen ftorfen/ein groß 3 Ungefium madien: wofelbit bas Diolgebirge Ecylla rin bober felis/ weldyer/wan die 2Sinde gehen und Dar: an ftoffen/ wegen etlicher Scolen / ein langroalendes Setjoin geben / alo bon unterichied lichen diffonirenden Stim men/fdrectlich anzuboren / uno wenn bie Sdjiffe ifnen zu nabe tommen im Sturmwetter / muifen fie serfobeitert meroen. Bleith gegen tiber if Der 9) Reerfollund Charybdis, on fich Das Meer berfenctet/uno gleich Dortben wies Der berbor broobelt mit fibrectlid)em Ysuten uno Siefabr ber fúriber fohifs fenden/Die fo wol Diefen als gegen úber. liegenden Sut meiben muifien. Daber Dasespridy wort entitanden:

Incidit in Scyllam qui vult vitare Charybdim.

Selbigen gefábrlichen Dit ju erfor: foben / befahl Der sionig Diejem Mens. foben fich in felben Sisluno binunter julaffer/uno bavon Seriegt ju thun. Uno Damit er befto wíliger / uno im binnunter fabren befio sewifier war/ lies Del sónig einen gulloenen plocal am felben Drt binein werffen/mit Zer: beiffung/ wenn er ifn wieder beraus bringen wurbe / folte er ifm verefret fern. Jiteolaus láffet ifm Das belieben verfpricht fein Dejtes Darber) zu thun/ machet fich freubig in Den Etrubel/und Eunpenadi Drew Diertel Stumben wies Det empor/dem Pocal in Der STand ems pur baíteno. Darauf witr el in bes fios nigs syaulaft eingefubret uno nachvem er als vou Arbeit ziemlish algemattet/. mit einer guten S)?abljeit erquicfet/ yor Den Ronig geftulet / Det Dami auffoes 
Sonigg Fragen folgende -2introort gitst:

Bnnábigfter Sónig / waz von Ew. Draieft.mir anbefoblen worben / habe ich verrichtet-2lbet nimmermelse baitte ic) Dem Rónigl. Sefeht nadyeominen mollen / menn icl) juvor getwuft brite/ rasa ich nun erfafreen babe / uno neerm ifse mir aud) euer halb Sónigreich hat tet verefren wo dlen. Denn es perno sier Dinge/fo Diefen Sort nicht alleine mir/

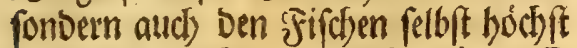
gefábrlich machen / nemlich I. Die groffe Bjemalt Des aus Dem tieffen Sdbluno beraufffahrenden orbafferz / welchem roie auch Der ftarcferte gu wiberftehen fichnicht unterftethen Darff/ alfo babe auch ich nicht vermodbt Ginourch ju Dringen/hate Daher Durch Nebenmege mich zur (Brund machen múfen. 2. Seernach fennd allenthalben fefret viel fpicige und icharffe SteinElippen/Durch weldhe ich nicht ofme Selens: Befabr uno Zerfegęung meiner. Saaut Den(3)runo exlangen mufte. Bum britten if ein ftarcfer Strom Der unter Srbifden $23 a$ fier / fo burds Die Telfen Dringen/ und Den aus Dem Shlund fteigenden 2Saffern entgegen arbeiten / auch fo graufam/ Daßs ciner fúr Furché erfiarret/ feerben móchte. Sttm vierbten waren fehr viel groffe ungefineure Polypi, Deerer seib Den groften Mann ubertrefien funte / fellige biengen an Den Seiten Der Sllippen / mit langen aubgeftrectiten

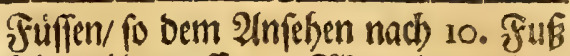
Eánge úbertraffen. 28 senn Derer einer mich ertappet bitte/mareid) Durd DaG Unnffangen toot georuceet morden. Biviforen Den nabeften Silippen fiellen fich anf biel ungebeure Neer: Saunde (Darvon oben pag.36. geoadit wortern)

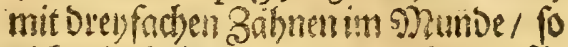
nicht viel Eleinet als Die Delphine / für Derfellen Ean niemanto ficher feyn/sanm nuen ein foldfer erfofnappes / barff ifm Feine Siechnung eines längern febens machen. Als nun Niculaus biefes or: Dentlic) erzeflet hatte/fragte Der fónig/ mie er Dann Den Becber fo balb hatte finden Eónnen? Darauff antwortete Niculaus: Der SJecher hatte wegen bes binund wieber lauffenten und fieritens Den 2 Baffer:Stromo nidht perpendicular oder gerabe Éonnen zu (Bstunde geben / fonbern waire fin uno wieber gur Seiten genoorffen worbern / lif er in eine flache und etraas aufgectyolete Slips pe gefallen/Dabatte er ibn liegen fefen und berauff getsolet. Dann wann ber Becber recht in ben 2 sitbel ober Scblund gefallen woire / boitte er ifn unmuglich erlangen Éơnen. E: z ware auch Dafelbit eine foldse ₹ieffé / Dápes Den 2 ugen als die finftere S2actit yorges fommen. Der Rónig fraget ifn / ob er wol nocb einft fich binunter wagen roolte/ fat er ztwar mit nein geantwortet/ als aber ein folcher \$jecher niederumb/ und zanar an einem Seutel boll Duca 
ten gebunden / bimein gewortifen nooks Den/bat er fith deffen belieben laffen / ift wieber binunter gefafien / uno nicht nieber berauff gefommen / vielleidst von einem Polypo ober Carcharia ers wiffert worden. Diefe Saiftorie gleich mie fie in Den Actis Deš Siónigez auffiges. zeichnet/ if fie von Dem Secretario Der Archiven Kirchero mitgetheilet noov: Den. Uno habe ids Diefellbige mit eins

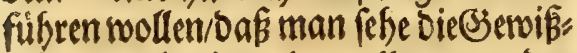
heit / masken Den Autoribus bon Den mehterwehnten Yolypis uno Carchariis gefagtwirb.

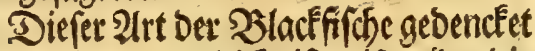

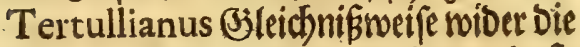

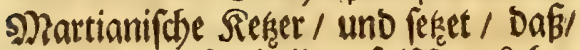
wenn fie mercten in ihter fallichen Sebre úberwunden ju fenn / fie mit Dunctein Einmuirffen und difputiren ifter Stra thume verffecten/o a pan man ibren falfotsen ङ3ang nicht feflen joul. lib. 2, adverf. Marcian. Sie bedeuten aud hominem occultæ naturæ \& dubiæ veritatis. Und in fpecie Der Polypus, weil Der fich an bie Şel fen fauget un feft balt' einen verfds lagenenen Menfchen / ben man nicht leicht fangen Ean. Sitem/ einen Sjeiksigen/ Der alles nach ficd reî́ fet/ und wab er cinmahl ertappet/ nicbt wieber los láfet. Ylaut, in Aulul: Ego iftos novi Polypos, qui ubi quid tetigerunt, retinent.

2Sab aber foiche 2 lact ober Suttels fifose/wenn fiefich reben laffen / vor fich felloft fur 2ingeigung oder SBedeutung mit fichbringen follen/ ift beym Cicer. de divin. ju lefen. Gubernatores cum exultantes Loligines aut Delphinos fe in portum conjicientes viderint, tempeftatem fignificare putant. Sie foflen Ungerwitter bes Deuten. Eben Der Mieynung ift auch Plin. lib. 26. cap. ult. und Plutarch. quæit, natur, 18. Wrifl man bie Deus tung auffe etwas weiter siefen / fo mag Der Scollánber/ Der Den Filch befdyries ben/ Felbite es Deuten/warumb Der Filch albar angetroffen/Daber Fónig in En gellano mit Sdbiffen ju feiner Wrieber Eunfft ins Sieich if erwartet worden.

Num. 4. G/t wie $e^{8}$ Diofcorides beforteibet / marinum animal exiguum. 2riro Hippo campus genant

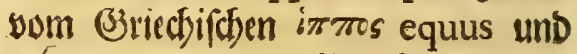
ха́нти eruca, meil eb forn als ein Wferd / und binten wie eine Bartens Piaupe mit fo vielen incifuris ober 216 . fäken: bat einen langen Sdjnabel Doer Siuffel / uno im felben gar ein Elein Mundlocb/Da es er Daburch Eaum etwas faugen Ean. 28Bitb aud vom Aldrovando unter bie Infecta gerechnet. Die groffen/ Derer wir wiel baben/fenno Eaum einer Şand lang / gar lieblich ans sufehen/aber ift Eein Fifoh / Den man ef fen Ean. Daßjer aber auff bem Na dien einen jubam Máfn ober Ram/ wie bie Wferde baben foll/ oder lebendig 


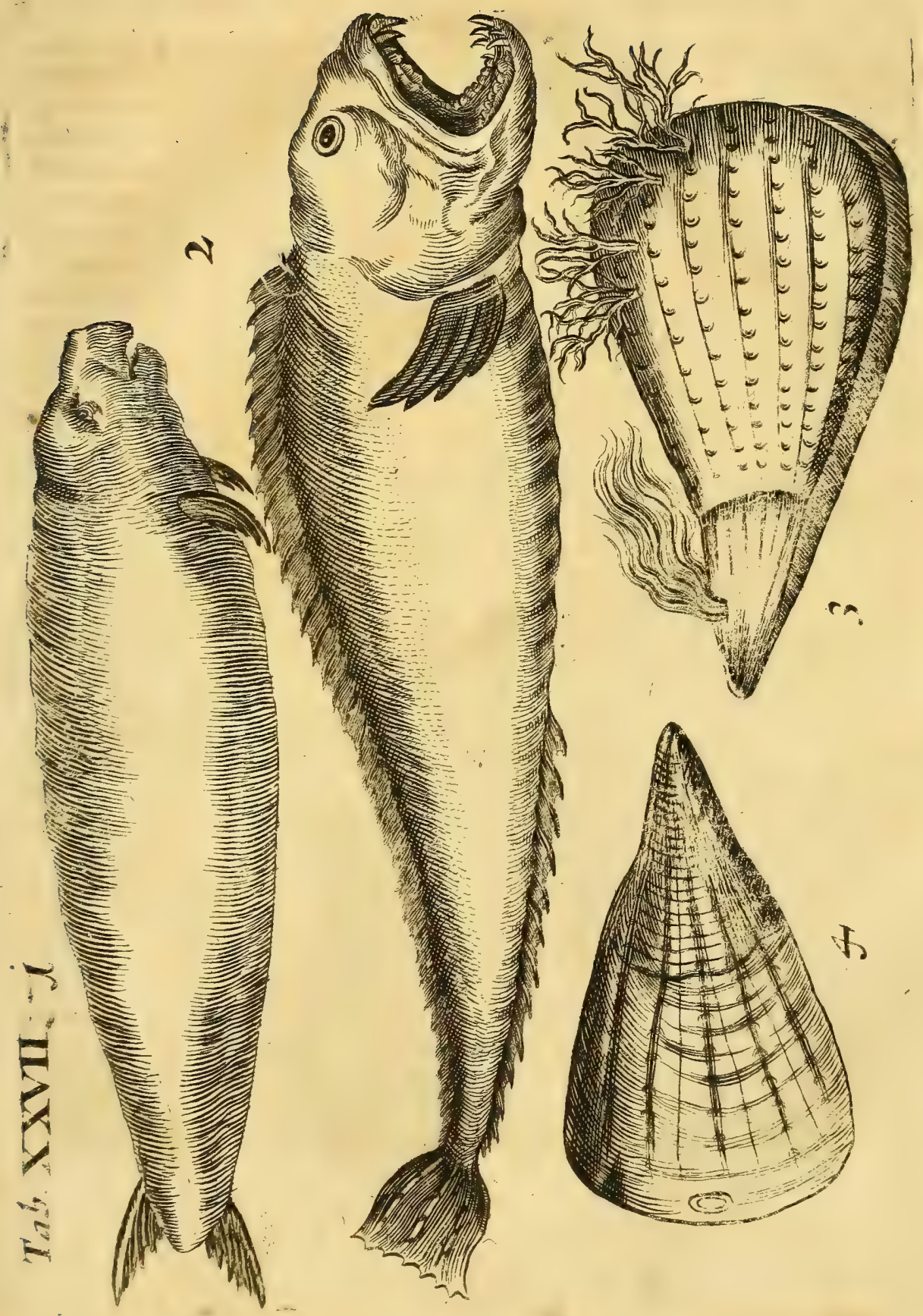



geflabt hátte/wie man (chreibet/Ean ich an Eeinem mercfen.(J)leich auch Gillius Fofreibet / Dafi er zu Venetien bey Den sifichern etliche lebenoig geferen I uno Eeiner einen Mláfn gebabt. Alian,lib. 14.cap.10. faget/ baß fein Saudd bers

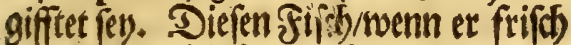
ift / follen leichtfertige ssenjoben einem Die Siebe bensubringen/gebraudsen. Aldrov; lib. 7. de Infect. cap. 16.

\section{T A BULA XXVII.}

Num. I. Ift ein Firf andertfall Eflen lang / bat Eeine Floffebern/ fon Dern forne an benden Seiten zweene runbe Eappen ober Zappen bangen/fein Maul ift einer Sube áfnlich/ Daber et auch eine Seefube genant mirb/ift von

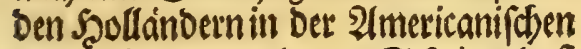
See gefangen roorden. Scheinet/Daf é̉ cine $\mathcal{A}(\mathfrak{r} t$ bes Manati cines in unferm

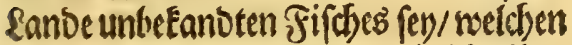
Clufius Exot, lib, 6. c.18. befchreibet/ nur Dafe Der Unferige Eeine smeen Fober" Sulfe unb auds nidte foldten Sdwants babe. MNit Des Gesneri $\mathfrak{B e f}$ hrei: bung aber fornter wegen bes @dfram. thes beffer úberein / uno miro von ifm aub Dem Rondeletio Phoca get mant.

Num. 2. Ein See 230 lffbat form im Nunde ohen unounten itharffe pisigis geauf Den Seiten aber swery Sieigen binter cinanoer runoe Sacten Bábnet. uno Dergleichen auds mitten im 2 adten. Sft ein veiffendes freffiges/uno ber $3 a$ ábs ne halber graufames Sfbier/in ber §ă tif́chen See wol befand/mirb oon ifnen Slipfifoh genant / uno ift gutes (b)e fotmacks/mic Olaus $250 \mathrm{rm}$ forteriber.

Num.3.4. 23erben Pinnx genant/ baben stoo Schalen gegen einanber grs

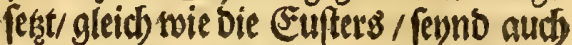

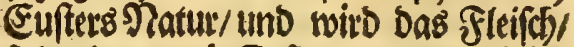
fo Darinnen/wic Eulters gegefien. Shre 2int it Spise im Sande ober Sdalicée ftehen/ fonft Eonnen fie nicbt leben / werben fo mol in Africa (mofelloft fie grôffer) als in Europas uno ponbertich in Propontiode, an ber Seite wo Nicomedia get ftanden/bäuffig gefunden. Sie eenno in gemein einen fuß / audis anderthalb

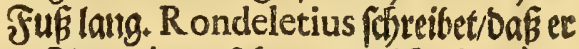
ju Siom eine gefehen / melche ben einer Ellen lang getweren. In folcher \&ánge baben wir aucheneseite/und ift 7 . 30 al uno einen balben breit. Snnwenbig am

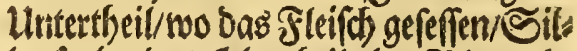
berfarbe/ Das Doertheil aber S3lutroth.

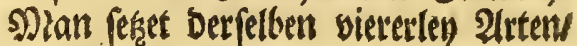
von Denen wir brenerten baben. STI etlidjen fol man Đerten findern / an etlis djen feyno Suefte als Shaare/ wie man an unfern 2uschelm findet/melche Ar:ftot. 23yffum nennet/ ourch weldse fie ifte Naherung giefyen.

Die Pinna war bejoen Egyptient (tefte Pierio lib +28 r) ein Hierogly-

phicun 
phicum auff bie/ fo in ber Sugeno all 3u fruffseitig anfingen fich im Yenus Spiel zu uiben. Dann bie jungen SMzuftheln fo in biefer groffen gejeuget - merben/follen ehe fie fich aus ben Scljas Ien begeben / iljer coitus oder Blifams menfügung haben. Daber nicht

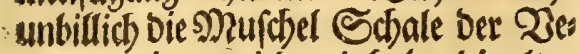
nus šugeeignet miro/und haben Die alten Joetenfabuliret/oie Zenus wáre aus Der SMufdel gebofren / und auff ciner Muidhelesthate in Eypern geforiffet/ seldses bod anbere zu Der falacitet, fo Durch Das Effen biefes Trifores vers uhrefachet riro / red)nen modlen / gleich swie man faget/Dap bie Euftern ein libidinofes Effen feyn follen.

\section{T ABULA XXVIII.}

Num.1.2. Sit eine fonderliche 2Irt bon Rreblen/fo bel) Den Smfulen Moluccis gefangen werben. Deree wir smeene haben/oer eine fo grofi als ein Mannes Sopff in Der Fiunde umbfans gen/Der andere ttwas Eleiner/aber zims lich plat/faben jocene Decfel//einen uns ten Den anbern oben/unb einen langen fpisen Sdhwantiman Ean nidyt felyen/

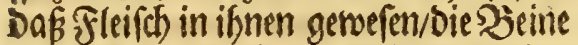
fernno fo Elar/ Dafiman faft Durch ferben faw/man fiffet audj nicht/ Dasfie Sche: ren gefhabe/ fonbern nur 8. Fúffe/mie bie Fúfie Der Tafchen = Rrebre. Diefe 2irt mus ben 2llten unbefandt gewe fen fenn / webet Plinius, Rondeletius nod Aldrovandus baben beffelben gea Dacht. Clufius ift ber Erftel fo ibn bes forrieben Exot.lib.6.cap 14.4nt' nach ifm Johan de Laet in defcript.Indix Occidentalis lib.2.cap.I9. viel eigents licher. Uno faget bas er mebr frufte babe als unfere Setebfe / erit 8. Eurgel Dann 2. längere/uno Dann wieder zwees ne Eúrţere/obne Sd)eren. Sçabe un ter ber er|ten Scbale efliche fleine SIlas Fen úber einanber/Die er als nie Die Frós fohe auffiblafen Ean. Er liebet bas $\mathfrak{u s}_{\text {s }}$ fer uno flache Derter im 23affer. Sift

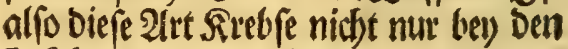
Infulis Moluccis, fondern auch in America am Fyflufe Chovacoet, oa ifn Die Einmoofner Signok, Die Utmerigen abcr(ppridyt de Laet, Araneum marinum nennen. Es if zu berswunbern/ DaßBAnno 1633+ein foldber Sirelo auch in Dennemarce bey seelfingor ift ges fangen morben. Es Ean aber fenn/wie Olaus 280 rm muthmafiet / Dáp er an einem Sallandirchen Schiffe/ Deerer Das mals vit aus Dof : Snoien wieber zus ruirfe gefommen im unten anflebenden Schilffi uno andern anbengenden materia berwicfelt/mit hetauB̉ gefommen.

Num. 3. Cancellus, weelchen Die Frarkgofen l' Eremite einen Esinfiedeler nennen/meil er in einem Bjebaiufe allein wobnet / if eine fonderliche 2Art bon Rrebfen / weldbe' in Sdnectenbaufern

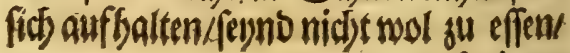
fondern 


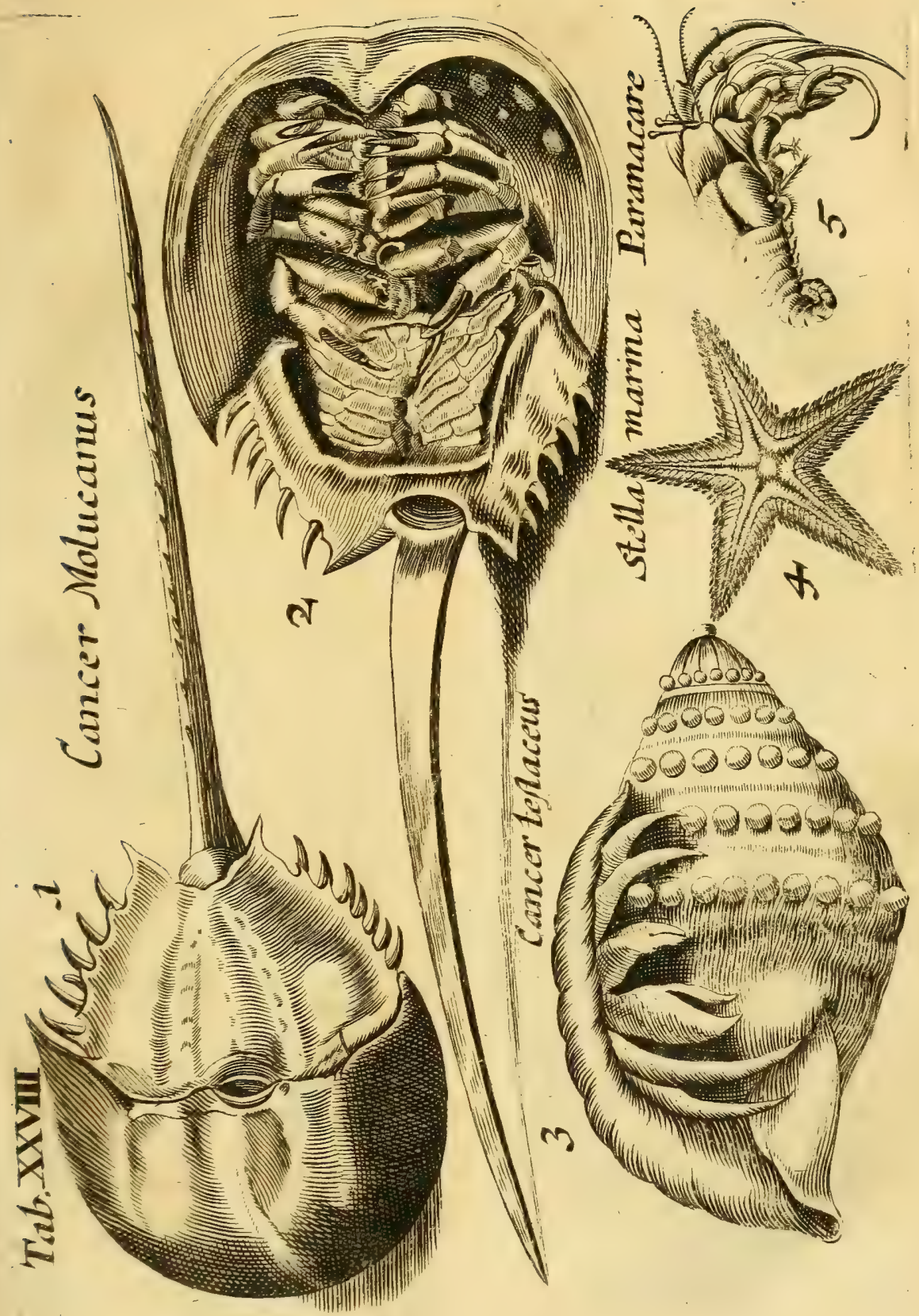





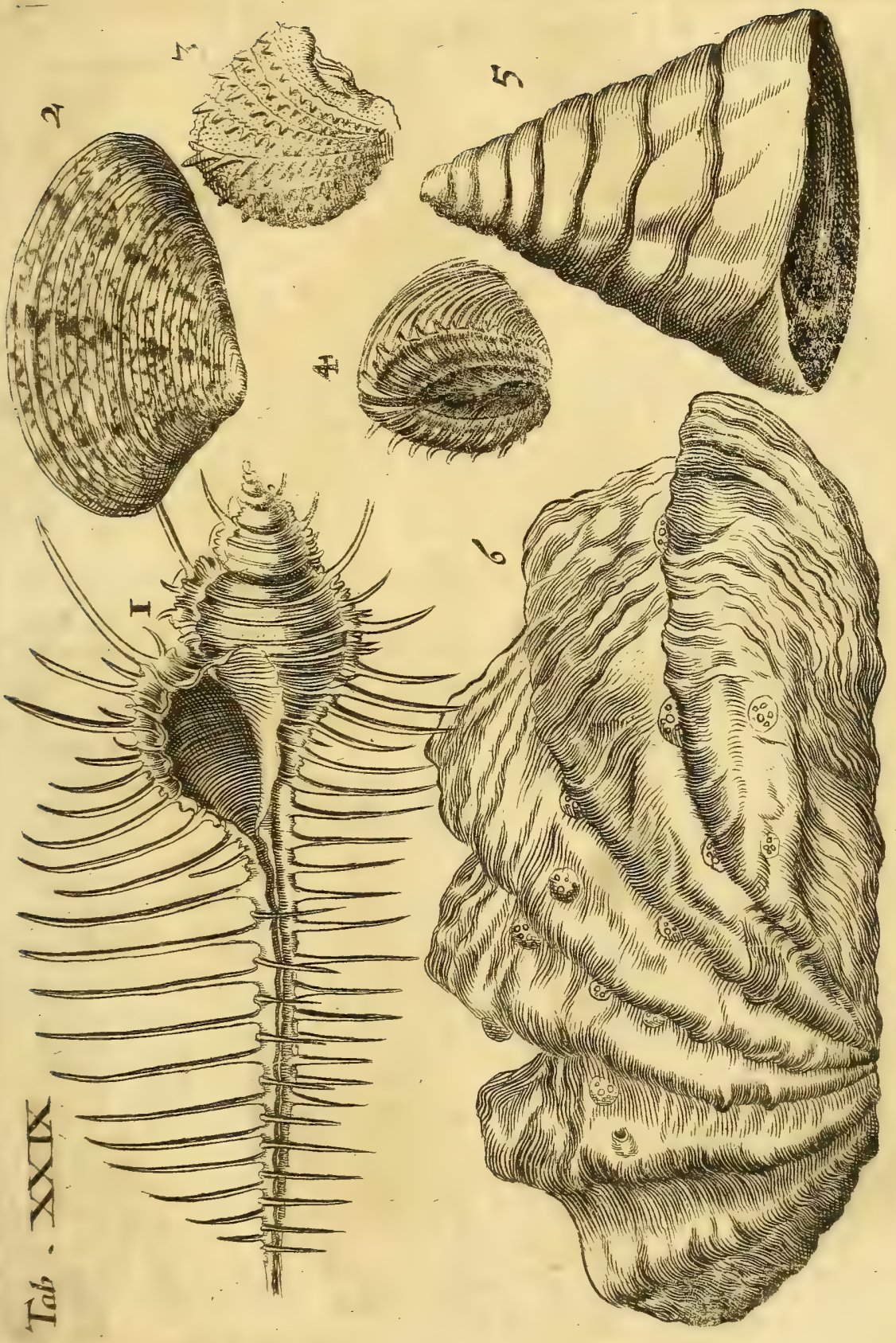



fondern werben bon ben fifchern gum Siffofangen gebraudjet / wenn fie von Der Sd necken abgefondert fenn: fie jols len in bie ledigen Sobnectenbäufert Erie den / wernn fie Elein/ und wenn fie ers wachien / bernus uno in ein gróffer Jaus fich machen.Arifot.meynet/Daß fie nicftrvie andere Rrebfe/fondern aus Soth und Sdleim gebofren werben/ aber fie haben gleichrool/mieAldrovandus obferviret / Ever an Der Seiten bangen/ moraus fie entifefen. Und befindet fich auds an Dem Unterfobeio

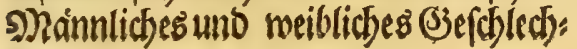
teg.

Num. 5. Paranacare butt ben St'as filianerngenant, Markgrav lib,4.c.2I. Sie ferno unterfisiedicher 2 rt Eleine uno groffe/bon den Eleinen babe ids viel in Savtlano bey Sdjevling am Stran De gefunden.

Num. 4. Sftein Neer:Stern/Des reerwir etliche baben / grop und Eleine/ wie Dann berfelben auds unterfchieds licher 2Arten fenn / etliche uno bie meis leen baben fünff Stralen / etliche fies ben/ etliche sehen / uns etliche stoólffe

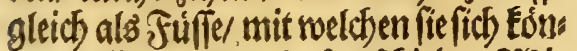
nen auff bem ङrunde fortichieben. WSir Gaben einen/ Der fehr fojodn formiret mit sielen Plucketn/bey einem fus im Diametro uno einer guten quer Sand boch in Der Mitten / hot auff allen Sciten Spişen gleich als Zálfone/oaber er auch Stella pectinata vom Aldrov.genant wirb / ift auffoem Shucfen mit Ereuks toeife- liber einanber gefdorencten Streiffen / fo albe ein Fifdjer Ses anju feben. Sin anoermit fo langen Radis. if aud Darben/meld)et auff ben Seiten Der Stralen anzufeben/alb menn er mit

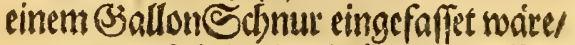
am unter Sbeile fentio fie gans offen uno baben Das \$lunoloct in bet mit: ten/wienol es fobeinet/o as fite ibre 9 ab: rung burch alle Etralen/tweldbe laingit binein bís sum Centro Bábne baben sieben Fonnen. Sie follen febr getne oie

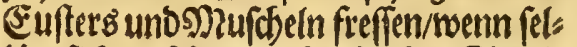
bige fich aufthun/ftecten fie eine Strale

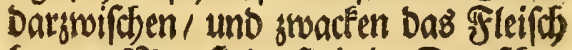
beraus. Szan finber fie in berDeutifien

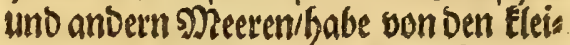
nenviel in Soulland auff bem Sandel wenn es Esbbe getwefen/angetroffen/oie/ wenn manfie angertifect / fich nadb Dee

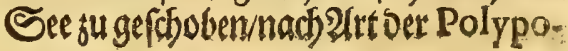
rum.

\section{TABULA XXIX.}

In biefer uno folgenden brey Tabu. len wezden etliche Teftacea, weeldhe audi. Conchylia genant werbenbefforieben. Derer Fleifh zur Speife ben anderts Exang vibus vom Aldrovando vorges zogen wirb. Es werben bie Sees Ibiere/fo Eein Sblut baben ( wie oben

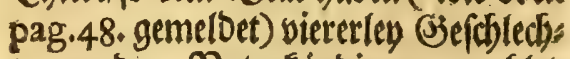
te von Den Naturtundigern etgeflet. (3) ii श16 
2118 Da fenno Mollia, Cruftacea, Teftacea \& Infecta. Mollium caro (ut funt Polypus frepia \& Loligo) duro ac ventriculo in obediens fereq́; abominanda hominibus eft, cruftatorum vero falubris,grataq; quin gratisfima fit, fi cum illis compares. Die weichen 2 lutlofe baben ein bart unberbeulich Jleifh/ fúr weldbe man (iic) huten foll / bergegen bie 2lrt Fifche to Schalen baben als Sireb fe/ fenno ges funoer uno liebliçer bom GSefomack' aber Dic Conchylia ( als Eufterz/-N)? (c)elm/Schnecten) úbertreffen aud Die andern alle. Denen bie Eluge und vor: fistige ? ?atur umb Eeiner anbern Uur a chen willen (wie eb ficheinet) fo barte Sauler uno 230 bnunge zugeeignet/als/ Das fie son ben andern Sees Shieren/ Denen biefe Speife aud angenebm / un befobabiget/Dem Menichen jur Speife und Ergeklichteit bleiben follen.

YSabi Die Sómerauff Die Conchylia(Euftern/Mufdoeln und Sdmeclét) gefjalten/fiber man beym Plinio lib.9. cap.35. Luxurie mater Conchyliorum pretia margaritis propemodum rquavit. Dafs fie felbige zu ef fen ifnen faft to biel Eoften laffer/als fie auff Jerlen getwenoet / Dann fie felbige bon fernen Drtern mit groffen UnEolten bringen lafien. 2Bie Seneca Epift, I. lib.i4. Daer ifre Untugeno fofilt//aget: Quantulum ex tot Conchyliis tam longe advectis per iftum Stoma- chum inexplebilem labitur? In vies fern fall feuno wir \$̧olfteiner biel glúcf: feliger als bie Siomer zu / hákenen / Dann wir fold $)$ Conchylia zur delicaten Epeife nicht fo ferne uno mit groffen Untolten buirfien bolen laffen / weil wir fievor Der Şbur uno frifch baben fons nen ( welches Denen in reit abgelegenen Dertern feblet) uno stwar umb fo fableche. ten Preip: an unferm Srte Daś I0O. umb 2. 4, in 6. (3rofejen auffis-bóchites bafi audi) gemeine Sanowercfis = \&eute felbige fauffen uno genieffen fornen / fo wol als groffe Serzen / bon benen Calius Rodiginus faget $(1.27, \mathrm{C} .23$. Conchylia adeo Proceribus gulæ probata, ut vel inde fit formatum proverbium ; effe Conchylia viduarum Cupedias.

Iher Diefe Speifen meitlaiffitig ju befdreiben/ift nicht unfers Dorbabens? fonoern laffen fie gleich wie allerbans fchón Slift ; alfo aud bie Poma maris, wie fie Tertullianus (de habitu muliebri cap.5.) nennet / ber Seerzen Sif the sieren uno ifnen wol fofmecten. Ung aber bergmiget jef̧o in unfer Sunfts Sammer Die leeren Sijalen uno Şrius fer ber foboinften uno rareften Conchylien in ibren naturticyen/gleid) als burch) Sunft ausgearbeiteten mancherley vers wunberlichen Bieftalten uno Sdjoin beiten anjufchauen/uns barinnen zu bes luftigen/ uno Den Scbopffer zu preifen. Uno gleids wie von ben Slómern bee 
Conchyliorum fleifon ( wie jest ges

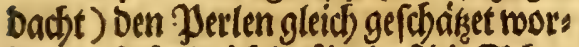
Den; alfo balte idb Dafúr / Dáp die Sda len etlidiser \$nufdeln und Sdjnecten wegen Schönbeit uno Siaritet Den ges meinen Jerlen / fo nunmefre faft in als Ien Stábeten und Dorffern am 23 seibs liden Sdjmudf gefunden werón/ no nicft úbertreffen / bods ifnen gleichen Eónnen / wenn man auds nur ibre Fat: ben / Die weber Der alte nod neue Appelles, Şerz Ovens/mit ifrem funnttis deen Dinfer lo boob bringen Eónnen / bes trachten wifl. Ulno bi if if bie Ulafachel warumb fie von siebbabern / wie man
Deret viel in Sodtano findet / theur an fich Eauffer mertoen. Dafer Saerz Sens Martens / ein vorneímer fouffimann in Fribrichftabt/ mein guter Freunb/ Der auch ein mol angeriditet Cabinet von allerhano fómen Conchylien und andern topltharen Raritáten bat; Die 2surbigkeit Der fidonen Conchylien I in einem Siákgel mit finmreid)en Zerfen befdrieben und zu erfennen ges geben. 2 selches iff nicht minber bem Jiefiker Derfelben Conchylien, als Den Conchylien felbft zu Efren mit bieber kescennial.

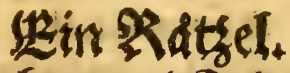

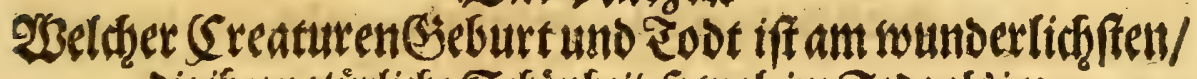

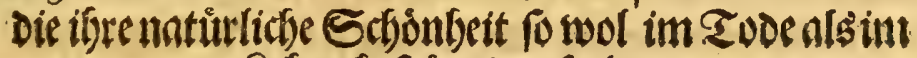 leben beftándig erbalten?}

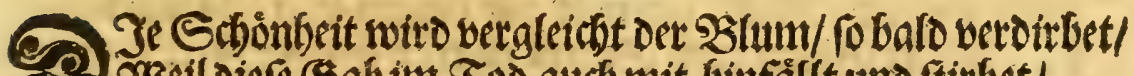

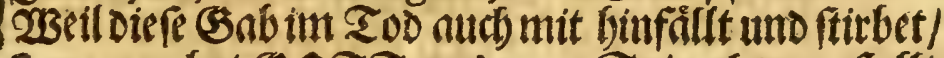

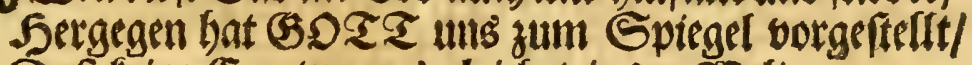
DoB Peine Crentur uns gleichet in Der 23 elt.

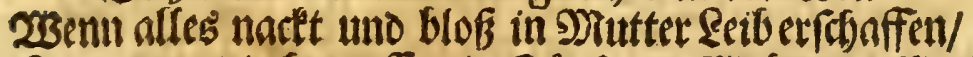

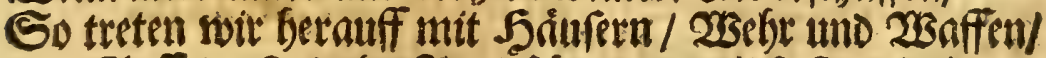
2(uff taufenterley Qitt / fefte wutmberlich formitt/ uno ofne Menfchen . Sand ro Eimftlich nubgesiert.

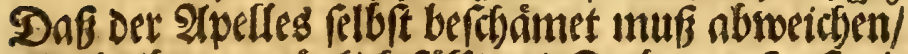

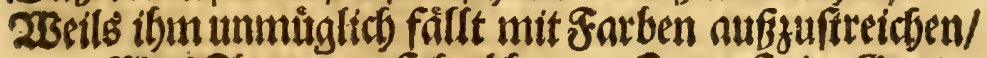

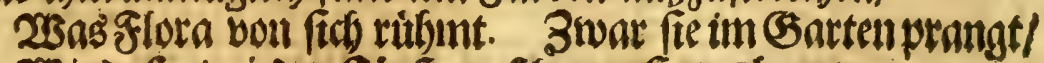

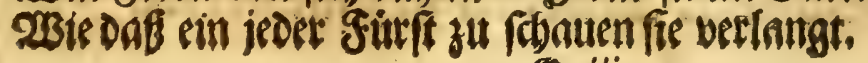


Sie bleibt Dod Eurke 3eit / ja oft Enum eine Stumbel

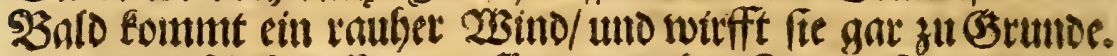
Woir bleiben für und fiul/uns erbet Rimbes. Rind/ utts raubet Eeine Zeit/Eein W3etter/Sturnt nod Wrind. So Ean গatuta uns Durch ifte Riturte fieteny Dấ wit atté nad dem Todtuntwandelbar flotiten. Jif jemano unfer शabm utto 2306 numg unbefanot/ Der tatbe was da blíbt/wenn Jyents fwingt Das \&and.

\section{Item:}

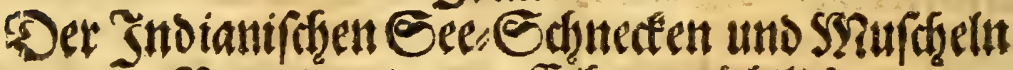
OSerantwortung auff ifte beroidstlidbe গnamen Begen bee 2 ofelgefteinuno perlenbobe

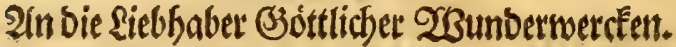

Se StPerlen uno Geftein/ wie initgt iffr Dod fo prangen/

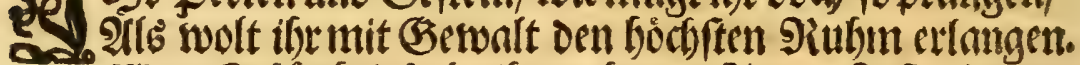

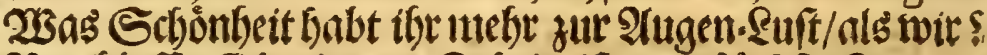

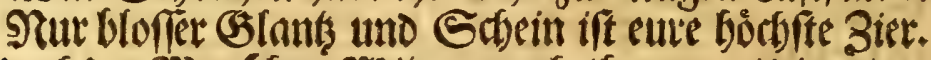
Den ood der פJen Daf cuer grofer Rubm for Gerslich triumphiten.

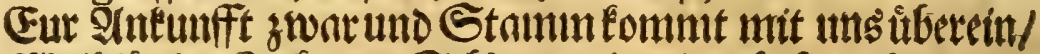
Weil ifre im Roth und Schlant wie wir gebobren feym.

Sont uns das witfte 9)eer ans Llfer ausigegoffens

2ub diefem tieffen (3rab ifs ja auth fero entiprofien.

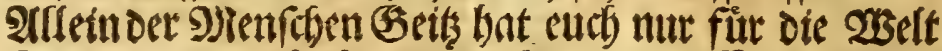

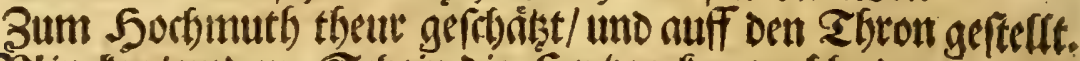
Eur Slincten/ und cut Scbein die Serthen Ean berblenden/

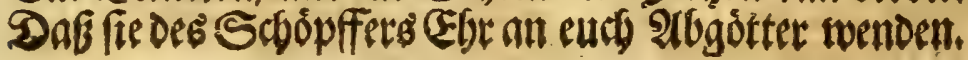




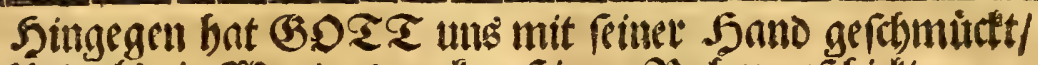

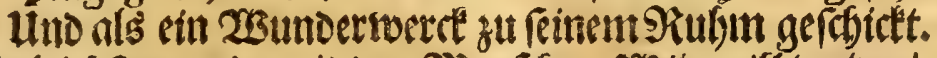

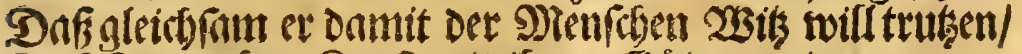

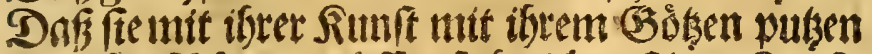

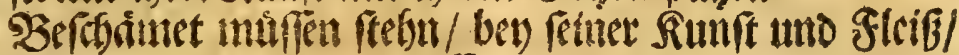

Damit er ung geztert auf Taurenterlen weib/

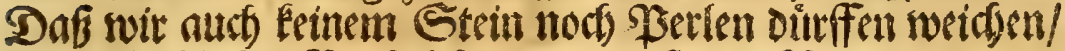

Sein Eimftlidber Pinfeel Ean ung perfect aubiftereben

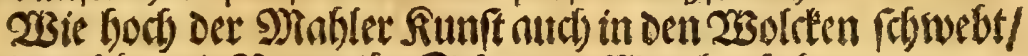

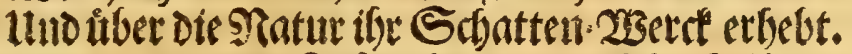

2rsir ftebn das gantse Inbt als unverwelctte Slite

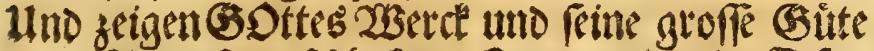

An unermféledten Etond/ die wir uffe fertno cin Spott.

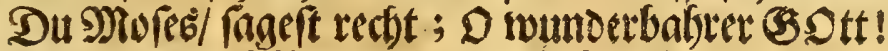

23asiman geringe boilt/ Das Eanftu fo begaben/

Dafi Mienichen boun Verftand daran Ergetsung baben/

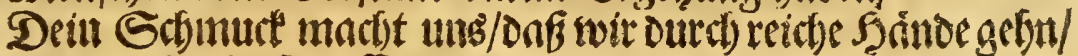

23er wolte fonft nuff uns verndte Sefhecten febns

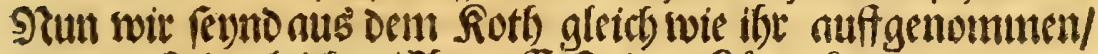

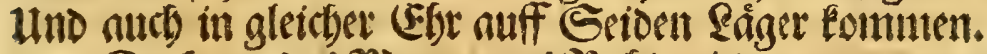

Doch wo des Dinmmong Sinbt wito gegent unts gefetst/

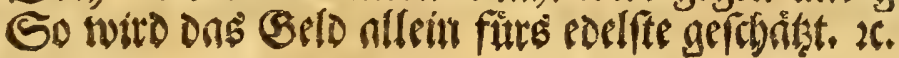

ร) (2).

Itno foviel in genere bon Scljoinheit uno Bierath Der Mufhein und Schnes c'en/Derer wir ungehflicbsiel und bie vor: nefomften in 8. breiten Eapfulen meif ibrer Raritát und Schónheit halber auff Seiden Suicher geleget baben / Da immer cine anders alo bie ander formis ret und fonderlich gejieret / Dáp manfich nichtgnug ứber bas sierliche ङiefchofffe Bjottes vernumbern Ean.

Num. I. Sof eine fonderlidje Art Edinecfen mit viefen fúbarfen Sta: chern umbgeben / Dergieiden Figur id) in Eeinem vor ben alten Autoribus ges funven. Matthiolus bat foft foldere 21rt pag,271,umb Aldrovandusp 284. abs. 
abgeseidnet / aber bev roeitem nidst mit fo viel Stadjelin / uno wollen fie unter Die Buccinas redinen / uno Эurpurs Schnecten nennen / pluribus fpinis hæc purpura horret, (pricht Aldro. vand. Woit baben zroar beret etlichel fo inwentig etwas Yurpurfarbe uno aufen weip/ auch etliche / fo innenbig uns auftwendig fohneeweis uno Elat feynd/Ean aber nidjt wol eine Buccina fever/ fondern vielmebr unter Den Cochleis iften Drt baben/ Davon anderswo gefagt mit'. Dann bieBuccinæe ober Sllafeborner feyno viel lánger / Die roir aud gros und fleine baben. Selbige als ein Slafehorn zu gebrauchen / foll Triton Der פ) fabuliren/erft erfunden/und bie Gigantes Darmit et/drecteet baben/Daroon bey 20efchreitung Der Buccinæ. Diefeß Schnecklaup aber ift feinem Einwob: ner in Der See ein feftes Sdblof und fichere 2 sobnung getwefen / weldse zu verforlucten mol eein Fif fich wito uns terftanden baben.

Num. 2. Ifteine sierliche Mrufbel/ bon auffen Duncelgehl / mit vier Xies men / po mit lidft uno fobruargegl un: feriffieden / aus Dem Puncte ber combinentz bif an Den Limbum fich er: frecter.

Num. 3. Sft aud eine reclite ஒut

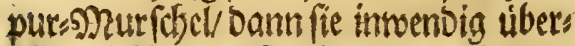
all bod) Plutwut: Farte/ oon aufien ges gen bem Cencro ift bie Selfite Wurpur mit $23 e i s$ vermifchet/ Die anber Selffte YSeis / uno ift mit erfoben puctiosten Streiffen úbersogen. Finde Dergleiden bey) Eeinem Die Sigur bezcictnet.

Num, 4. Diefes if eine gar sarte

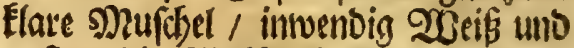

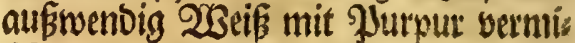
fobet/ift vollet Strigis ober Sunkeln einer neben Der andern / unb bat umb DenDit/oafie bzeit ift/charffe fpigesta cheln. Aldrovandus nemete fie Defs wegen Concham Echinatam cine Sgel:-9) fibel/weil fie fefrer rat/ift in sollanto umb 30. Gúlben vertauff nurben.

Num. 5. Sit eine 2irt Sefnecten von ben Turbinatis. Est werben abet Die Turbinata befdrieben/Dap fie langs lidft und in eine Epize binaus geben. Uno werben berfellen beym Rondeletio, Gesnero uno Aldrovando vielers ley Species erjeblet ( von benen roiro biefe Trochus Pyramidalis gentant) wegen feiner Sieftalt/weil es einem Reus fel/mit welchem Die Sinoer fpielew efyn(id). Darbon Aldrov, pag. 363 . de Teft. fennto von auffen alb bie Jerlen d:

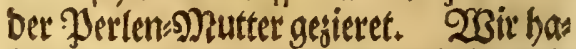
ben berfelben unter fobied lidse/ Eleine uno groffe / bie grofite ift fedfiteballs zoll lang. 2(ud Gaben wir von ben fleis nen/Die nicht weib/fondern eiferfarbe/ fo fich auff blau jisthet. Gtem anoere/wels

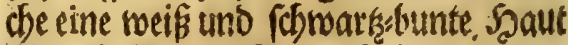
úber Die Jerten: DNutter baben. 
Num. 6. Sfteine Jnbiantifbe Eu: fter / vom Aldrovando Imbricata ges nant/ wegen ibser Eseffalt 1 Dap fie roie Die Sabhl:siegelm / Die manúber die an Dern ₹ad = Biegefn fruirket / uno in Sadjen und Meififen gar getráuds: lid)/ansulfhen. Plinius nennet foldse 2Att Euftetn (lib, 32, c. 6.) Tridacnam, Dem audf) Rondeletius folget/ meilfie nifft als nur auff brey Bififen Eónnen verfblucfet merden. Die fo Aldrovandus alggeseidgnet fot / foll nur

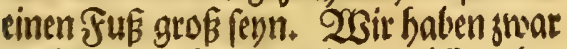
Derferleen auct) in gleidser (Jitóffe / aber ettiche fo úber 2. STußs / und eine meldjes Die ङstófte/3. Jü und einem ballen im Diametro, im Utmbfang aber / weil fie oval,8.ร us und einen balten / und hat ein/tarcter Mann su thun/Der ein Sheil vonder Eroen aufheben foll/wigen beys De/ fo jufammen gefóren 467. Pf fund. Es Eónmen in einer Schate brey ober vier netwe gebofone ßinver liegen / Die ein Daš anbere nicht anruibret/ift benen fo es anfangs nur gehóret/ungláublic) vorgetommen/bis fie es fellofit gefehen. Eelbige Eufter Schalen habe id Anno 1655 . in Encthufen von einem Spto Sndienfabrer gefauft. Sfft: in Solt: Jndien vor (Boa mit Dem 2incter auffis Edhiff gejugen morben. Sin ibe Fleifo follen fich 120. Perfonem/ fo auf Dem Sdhiffe geweren/getheilet/und \&uft halber Davon gegeffen baben/fol aber gat bart getwejen fenn. Diefe mare etwas gróffer/als bas fie auf brey Sif fen Eunte verfotluctet und Tridacna genennet werden. Sie follen in Ins bien nod) gróffer gefunden werben / wie mefreerwefinter Bseorge 2anberfen in peiner Эieifebeftseribung Darbon S?ad): richt gibt/menn er alfo fisreibet: Unjer etliche/als wir bey Den Wrinkens Enlano vor 2 Indfet lagen/fubten mit einem $\mathfrak{B O}_{0}$ the an Das \&ano / umb Verfrifterung und Salat/ wellden bie Syollánoer ifs: rer vorbey reifenden Schiffe balber Das bin gefáet/ zu boten. Allz wir an ben Strano getreten / faben wir eine pebse groffe Eufter/fo nod) halb im 2 Baffer lag/war 7.Ellen in Der Siunbe/ werben von Den Soullándernsilipeunten genants weil fie fich gemeiniglid) bey Den Rlips pen befinden. Z3u jelbiger Eufter lieff einer von unfern Wólceern / umb su fefen/waber für ein Sthier: und als fie fich etwas auffigethan / meynet Der gute Menfíd fie waire entweber tobt ober Doch Siraftlog//fectet Die Şand binein/ Das Jfleifh anjutaften. Die Eufter aber thut fich gef fohminde rwieber su/ uns Eneipt ibm Die STand ab/Dann bieSchas len am Эande sheffers fobarffe baber.

\section{TABULA XXX.}

Num. 1.2. Echinus marinus fpoliatus à fuis Spinis,wie fie Aldrovandus nennet/fonft Sees?pffel und Sees Jomerangen/weil fie alfo geftalt/follen $\mathfrak{S}$

tefte 
tefte Aldrov. p. 405, de Teft, umb Porwegen uno Dennemaref fo wol als in Den Auftrialfichen Seen gefunden werben. Man foll fie (wie Strabo lib,16. (chereibet) an etlichen Drten als Shute grofi finben. 2 Bir balien von Den Eleinen unterfohieolicbe 2rten/

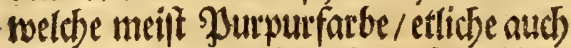
boch roth und etliche weis. Die Wur purfarben faget Nicolaas Perottus, baben im \&eben bie gruine Farbe mit un: tergemifchet an fíd / melche Den 2lugen febre anmuthig fallen/ uno roenn fie fol d) Farbe befielten / nulla effet cum gemmis comparationitbertrefen fie bie Eolgefteine/aber im sobe berlieren fich Die Farben etlicher maffen. Der do gebachter und bon uns begsechneter grof fer Sees/pfiel/ ift fo Eunftio und licbo lich/und viel fohoner als er fat bezeichs net werben Fonnen / anjufeben: IIts swenn ber fuinjtlicb/te Jjerlfticfer fein Mieifterftude Daran batte wollen feben laffen. Was Die Scljalen foldjet Seestpffel in ber Medicin für Nuten baben / ift in Olai Wormii Mufroo pag. 26I. 3 lefen.

Num.3. Sfteine Mufdrel/Die ibten Drt wol zieret/unb werth ift / Dẩ man fie babe/nidt allein megen ifrer Sobon beit $/$ fondern auds wegen ifren Ges brauch und Sugenb / bie manifr jus forreibet: wiro von ben Naturtúndis getnConcha Venerea genant. Et fanè (inquit Aldrov, ) aे Pulchritudine fplendoreq; \& lævore, qux dotes veneris formofiq; corporis præcipiæ funt, nomen hoc merentur.2Seil fie fo fdoon als bie Venus auffgepuket ift. Gesnerus inesnnet / Daf Diefe Muufdel Den Namen son ber Venus baber bes Eommen babe: Der Syrannighe So: nigzu Corintho Periander (gleich) wie $\mathfrak{E}$ Plinius lib.9.cap.25. er'seblet) babe einft einen Soten zu 20affer auss gefobicts/ in einer gewiffen Stabt alle Ioeliche Sinaben zu caftriren / Es bat: ten fich aber viel folcber \$nuforfin un ter an bav Sdbiff gefogen / gleich lvie bom fifche Remora gefaget wird/Daf Das Schiff oor 2 Binoe aud fille ftefon múffer/uno alfo Die Pooft zum Unglice Der Gungen Sinaten ware verbinoert rostuen. Sababe man baber biefe 2irt 9lufbefn in Der Venus Eempel fu Gnido geefret/uns fie nach ifsem ?as men genennet. Diefe Shiftorie ift eis ner Fabel gar efonlids. Die Franţo: fennemen fie Porcellaine, úber wels chen Namen fich Aldrovandus mit Dem Zoographo verwounbert / uno mennet/es múfe bielleidjt â porco bers Fommen / weil Das soch auff ber einen Seiten cinem Sdfweinuiffel etwas efnlich. STsarumb nicht bielmefye wegen Der gankęen aportur, twenn man nil geiten laffen war Varro faget: Mulieres noftræ;, nutrices maximè, naturam, quâ foeminæe funt, appellant porcum. Schb balte aber/Daß̧es 

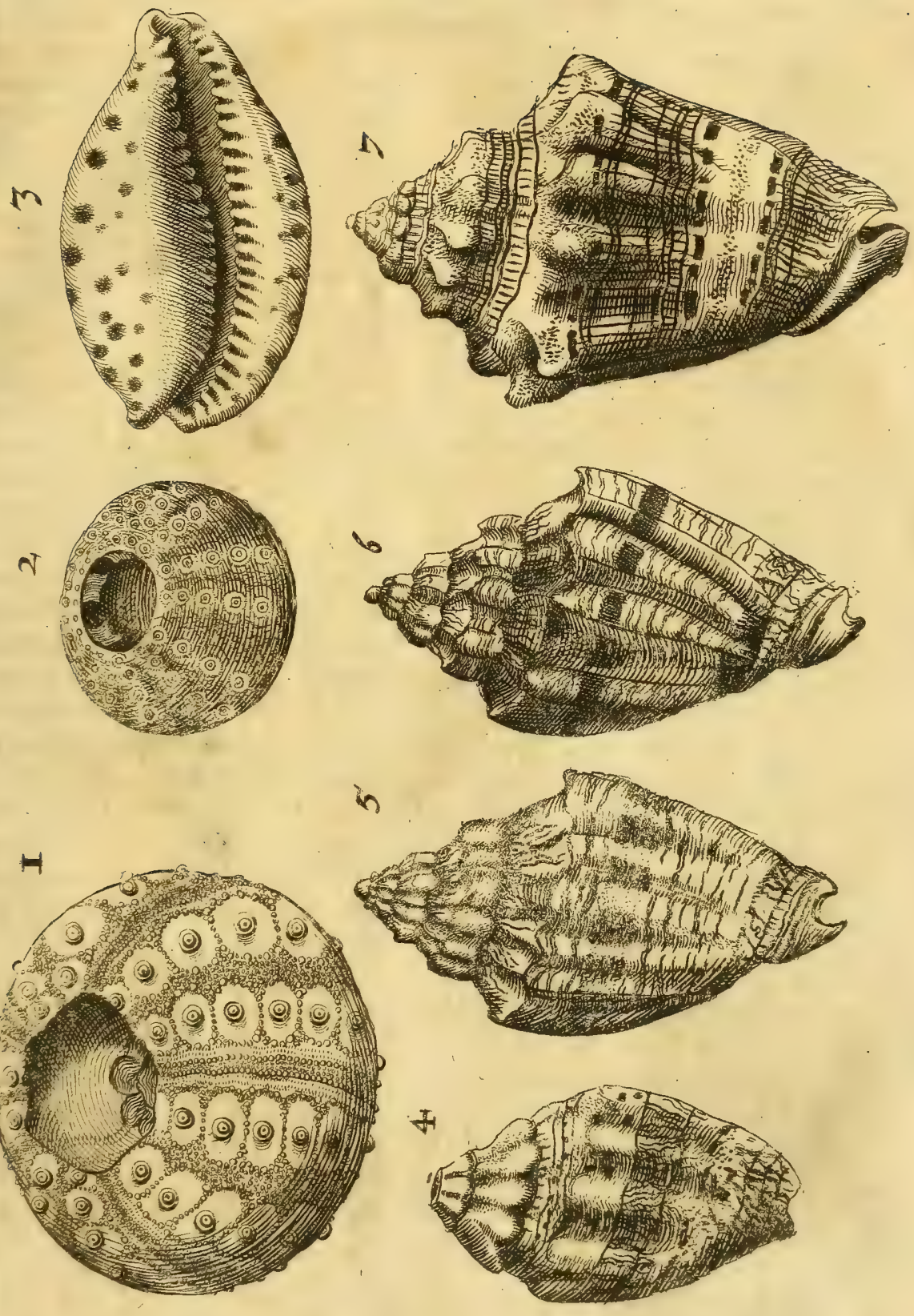

feu negen Dem eufferlicben Anfeben Den Jorcelanen Ebinefifoden Befdirren efonlich. Dann etlicbe feyno úberall Schneeneif unb belle/ intoenoig Jur: pur (fo fefrer ) bet wir aud eine bas ben. Diefe werben von Den Ethiopern, fo fie Buzios nemmen / ubltalle D) Raffe boch gebalten/Daf fie umb felli: ge/torif nidst wie viel Buolo/la offe gar bie Sltert ifre Rinber vertaufdoen/mel () ben foll / oa foldse Eleine Sorufdoln an fat Des S3eldes geluraudjet roerben / tefteAldrovand.de Telte pag.559. St: liche baben fobmarkblaun eingeforengs: te runbe flecten als srofen grof̂. Gie fenno ingemein als die Sanfe Fy: er etlicbe nur als 2 Sallnuife gros/fo ges meiniglich braungetb auff bert Fucten mit weiffplenglituten flecfen: (5tlich)e umb Den Sippen betumb fómarks / uno auff bem Srucfen braunroth. Dergieis doen wir auch eine sar groffe baben / in foldser. Bieftalt / miefie Gesnerus bes fotreibet / uno Porcellanam ruffam majorem neinet. Sie follen gar bauffig im rotben Meer gefunden / oon bet Eintoobnenben geff fchet unb sand lung Damit getrielen toeroen/wie Bellonius ber es gefeben/berichtet.

Man pleget fit nicht allein in Stas

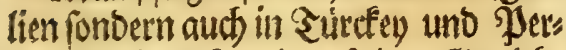
fien ju gefraudber/ Das \&einem:(Sieratbe

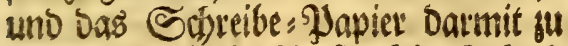
gletten. Uno iff in Perfientem Sqreis ber ober Sobulmeifter/ Det nicbt eine folde Sorufdol unb bas \$apier ju glet: tely/bey fich tragen folte/Daber nennet fie Bellonius Conchaslavigatorias. 2Sorzu folche Venus - Mrufditn fonft gebrauchet toerben unb auch oiens (ic) fesno fintet man bin unb wieber bey Den Scribenten. Aldrovandus faget cap.82. de Teftat.Daf in Sytalien Die unguidytigen 2 Seibes @erfonen Die Eleinen Venus Dafdoln in filbet uno Siolo gefaffet am Salfe tragen/unter Dem pratext, Daf / weil fie wegen bet (Se)talt Uterinæ genant toetben / wibet Die Mutter Srancfbeit/ als ein amule. tum am Salfe tragen / aber ibnen nut ein Seoet Stank feon lafjen/antubeus ten/mas für 2 Bein bey ifnen su Eauffer uno locien alfo ibres gleidben Sinnes Seute fu fich. In bet Medicin, fdret: ben Die Medici, follen fie gebraudoet verben / ben Şlutfufs su fillen / uno Das (S) fof wer in Den Sermen subeilen toenn es pulberifiret eingegeben wirb/ fonbertic fol es gun Babn pulfer gut gu gevrauchen febn. SBenn seffel bats bongemadjet/ uno benen fo eitt trogen Rincebulten baben/Daraus su trincfen gegeben wirb/foll es gut thun. (Vide Olai W.ormi Mufæum pag.252.)

Man bailt es aud Dafür / Daf biefe 9) Rufobel ein nustich stincfgefobir? fenn foll/fir oie jenigen Jratwen/ bie in Rinbes ?dotben arbeitew wenn fie ben Woein/o nur ein wenig Darinnen geftans मू. ii 
Den/baraus trincten/ foll Die sicburt be: fobern / welifies etliche furmefme Plers fonen in Diefen \&anden in Der Wrobe gut befimben / Daber man fie in Silber uns solb eingefalfet uno auff Júffe geterl. let.

Num. 4. 5.6.7. 2Serben Murices aud bom Rondeletio Turbinari ge

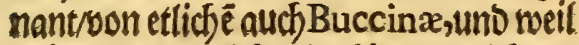
etliche / fonoerlich Die bier verseichnet/ fteben/tbeils mit 4. theils mit 5. neben einanoer ftebenden sinien umbgeben/ unv Dargwifonen vierectite fojmartse; Num.5.aber rotbe Flecfe orbentlich als Şboral Moten geferet werben / fie aut Conche Muficales genant. Des rermir Bstoffe und Sileine baben. Seyno gar fochón und anmutbig an suffbauen.

\section{T A BULA XXXI.}

Num, 1.3.4.6. 2Berben Cochlex Cylindroides genant / feyno peft fchón glat uno matmoriret. Num.I. mit braunen Streiffen auffeinen weif fen Strund und cingefprengten geblen - Flecten. Num,2. 20seiffe Flecte auff (6) warksen Grtunt. Num. 4, mit Duncfelbraunen Flecten. Num.5. ift gans gebl mit weif marmorirten 2lbéken. Num,6. an einet Seiten Eeibs farbe und bernach braunlich auff meifs fen Biruno, Nody andere in bie funfs 委igerley Arten von berfichiebenen For: men und Fatben feryno verbanderv/ Dafi wie bie Natur fo wunberlich fpielet/man nicht gnugfamb befidreiben tan.

\section{TABULA XXXII.}

Num. 1. uno 2. 2 Berben Pectines genant / fersns geftreiffet mit erbobenen Falten yon unterfóieslicher Bitóffe uno Farben / Siothbraun / Pourpur / (seelb/ Siothbuno/ddyefien alleseit grweene auff einanoer/Dic eine erboben / Die andere plat/gleids wie Die Euftern: etlidhe bas ben Sappen an Den Seiten wie Shren/ Dabet fie aud Auriti genant wetberl. Die $23 a l l$ fabrtšşruber / wenn fie boné ompoftel wieber zurticfe formmen/ baben gerteiniglidy Derfelben an ifre Scute gef feffet. In Scollano babe id. gefehen/ bapfie etliche effen von Conchylien uns ander Speifen Darinnen bereit zu sifdre gebradts.

Diefe Art Eufters follen ben Den Al ten in foldsem 2 Berth gebalten worber fern/ babkein Convivium anfebntich genefen / wenn man nicht bon foldser Art auffgeferet / uno follen bie/ fo bey Mitylenen und Tarento gefangen werben/Die beften fenn. 2Bie Davon Horat: Satyra 4.

Pectinibus patulis jactat fe molle Tarentum.

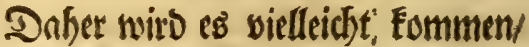
wie Aldrov, mevnet / oap Die Taren-

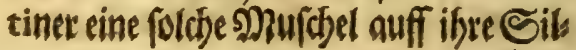
bern 

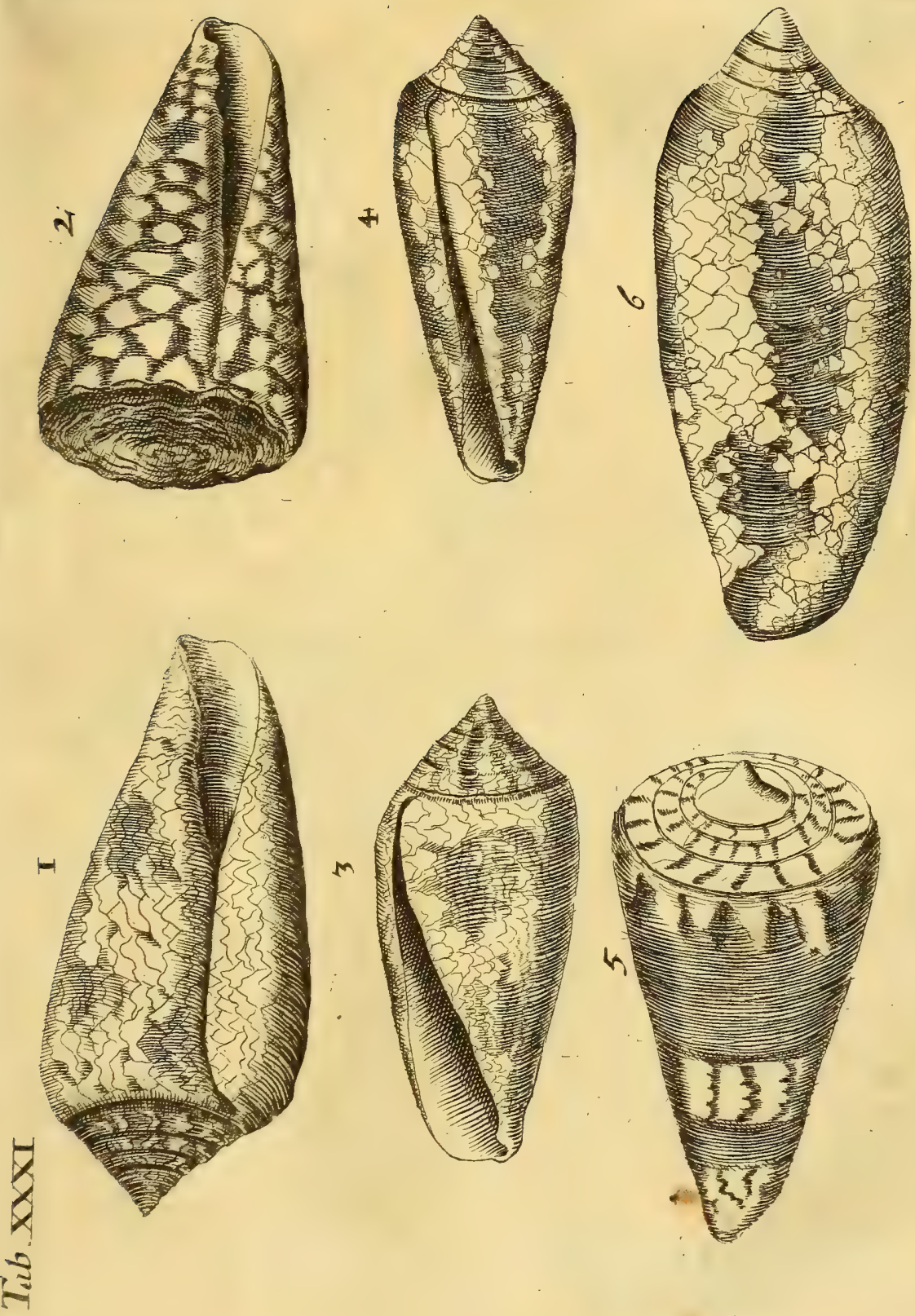



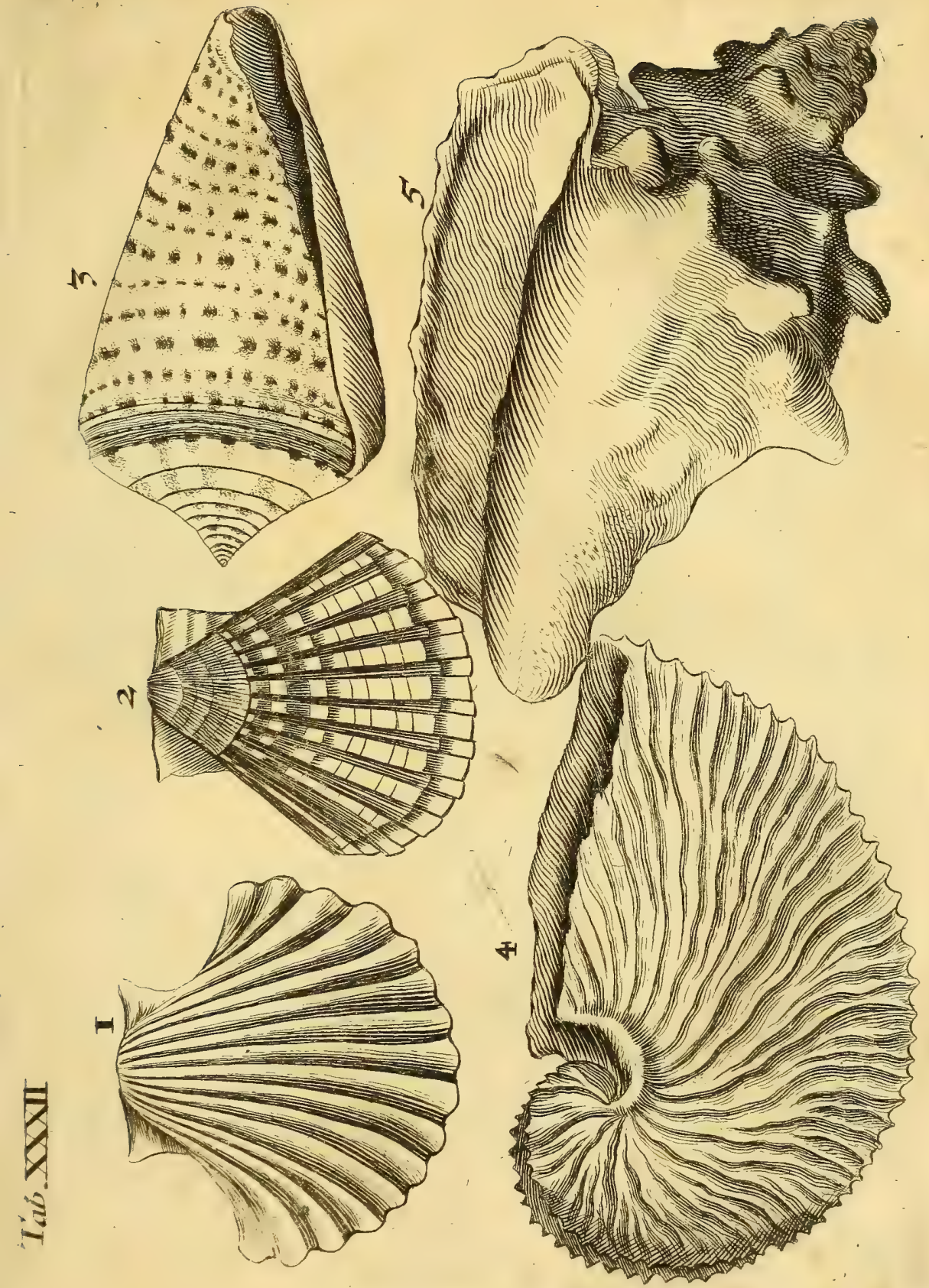





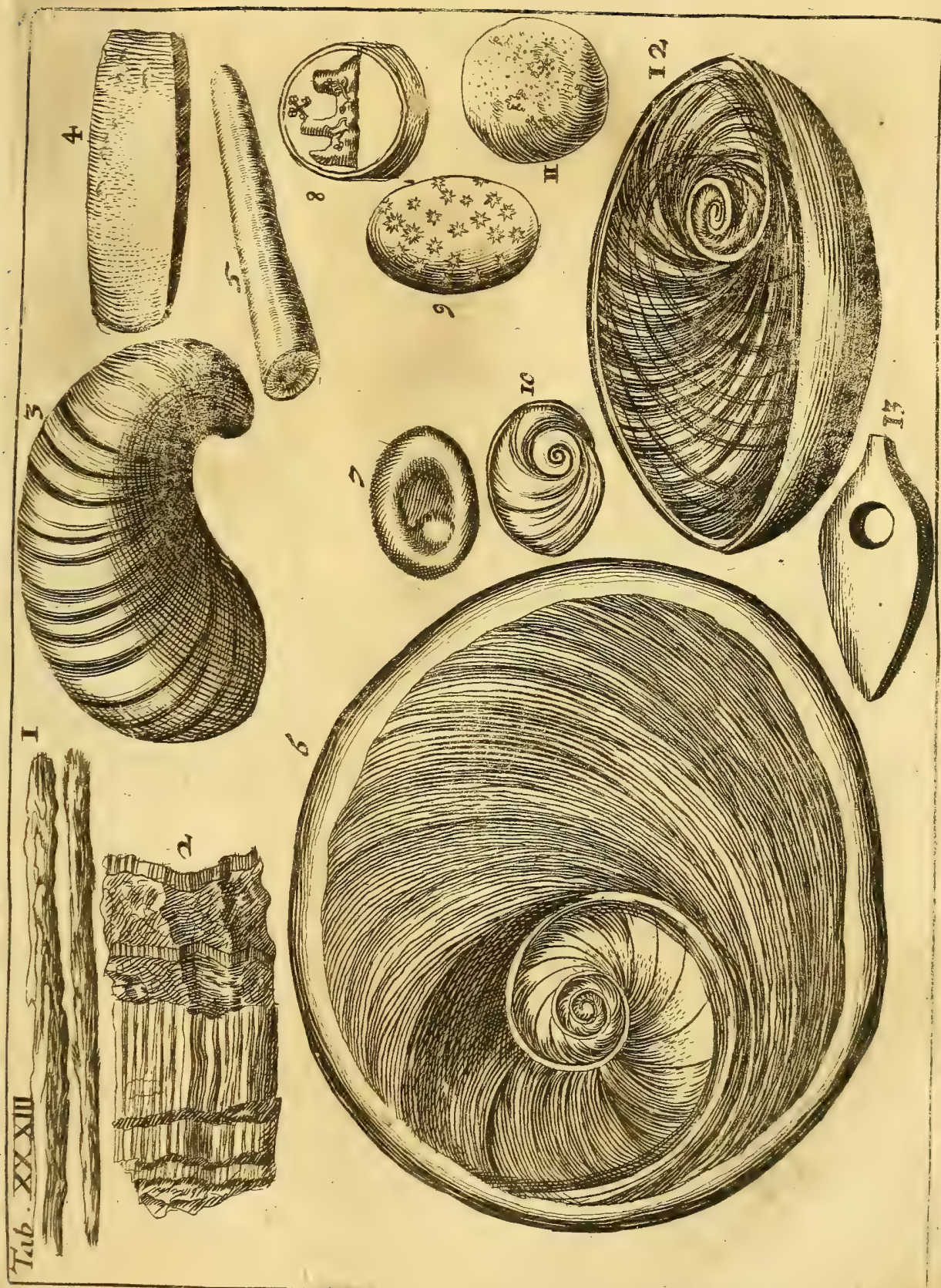



bern Munn gebrauchen. Xenocrates (tefte Aldrov.) meynet / Dapi fie lieblidjer fonmecten / uno leidjter als bie anbern Eufters su verbauten feyno.

Num. 4. Sft ein gar zartes/ ounnes uns Elares (S)ebáufe ( nid)t bicker als Pergament) eines Seethiers/ weldses Nautilus, von Roberto Conftant. Nauplius, Yom Plinio lib.9.c. Is. Pompilos genant wiro. Es foll faft eine 2Art von Den Eleinen Yolypis ober auch Sepia fenn. Plin. 1.9. c.30. und c. 29. ppridft Plinius : Interpræcipua miracula eft, qui vocatur Nautilus ab aliis Pompilos. 2rseil es wie ein Schifflein formiret / mit welchem fit Der Fif bie şobe heben und auf bem 23 affer als mit einem 20 othe bor 23 inde fah:

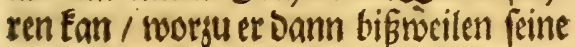
2trmen auff benden Seiten ausgeltites cfet als Oruber gebrauchen fan. Utno wenn ein Sturm fich erzeget / Eeffret er fein Schifflein ganş uแnb / Dá baš un terfte oben zu ftehen tommt / Damit fein Scbifflein nicht boll $23 a f f e r$ fodlagent uno wieber zu Situnbe gehen foll / wie Elianus fobreibet. Merefet er aber/ Daßer auffoem 23 affer verfolget wird: füllet er fein Schiffigen boll $23 a$ afert uno verbirget fich roieder zu(3runo.

Num. 3. Ift ein grofi Murex úber eine balbe Elle groß 1 uno inmenoig

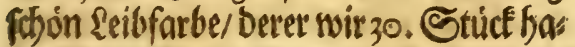
hen meroen vom Aldrov.c. 9+ de Teft. lib. 3. bef(hrieben.

\section{T A BULA XXXIII.}

Num. 1. Diefes ift von einemङtét ne/ ber Alumen Scisfile genant/ miro aucb) yom Boetio Schiftus genant/ ift grunlicht / wenn man ifn aber geflops pet/ wirb er meeis / und Eanman ifn in einer Sel = Sampen als ein Dacht ges braudhen/ welcher nichtberbrennet / uno alleseit gut bleibet / wie ids z Dann felthit probiret/und alfo Den Cordanum wabe befunben. lib. 5. de miftis, wirb nur weiffer Durdhe Feur / gleid) wie Das Talckum oder Marien:(3lap / fo Die

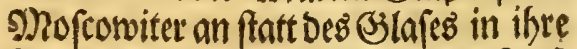
Fen/ter gebraucten/Darbon in Der Mof

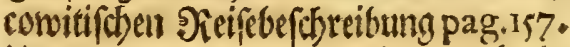
Utno meynet Boetius, Daß eहै auch ein Species Deffellben fey. Mhan Ean felbett Stein twifhen Fingetn zerteiben/ uno soent man einem etwas Darvon ins

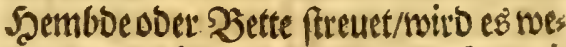
gen Der fpisigen fubtitem Stacheln eis neneben fo peinigen/als wenns bie rats hen Fafen fo úmb bert Sanbutten Sa men Yeum/ober Deffeln wären.

Num. 2. Sofi ber notable Stein Amiantus, fo auch Asbeftinus / weil er in Feur nicht Ean verbernnet werben/ genant wiro/fihet Dem A lumini Scisfili nicht fert ungleids an Der farter uno fets ner qualitet,nur dấ Alumen Scisfile feine 2ldern in bie \&ánge auffuifuret/ A-

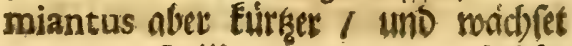
Sh iii

化iobers 
forichtweife broifhen anderm barten Steinen/wie unfer figur anoeutes/ba Das weiffe ben Amiant, dab fajwar fae abez Den gemeinen Stein bebeutet. Seis ne sugeno iff audb/oa verberentie/ Daber wirb ex vom Paulo Veneto 1. I. c. 47. Salamandra ges nant/ uno vom Plinio linum non ardens. Die2llten haben eś Éóñen bereiten/ baf́ man es fpinnen uno Suicher Dars

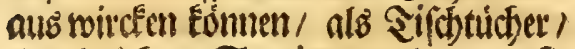
Scanbricher / Serbieten/ uno wenn fie unfauber gemorden/hat man fienut ins Fener gerworffen/ uno außsbrennetr lafs

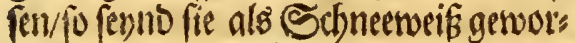
Dew/audl baben Die Seenden ifreen Eeis c)en umb Die Sdjam foldhe \&einmano gebunden/wenn fie folten verbrano wers ben. Die Sónige hat man gan de Sticher bunden/und verbrand/ bas mit Des Corpers 21sche nicht mit Det SJolkalfche vermifchet muirbe. 3u Эom foll ein fold) Sudf renn/in weldyem bes

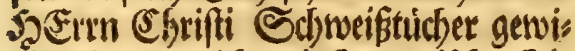

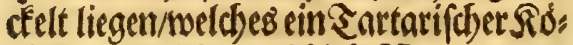
nig Dem Palft sugefthickt. Venetus diCto loco. Deñ andere Eeinwand wuts be fonft ebe als Der Córper/vom Feuer verzefyet worben fenn. Mran bat Diefen Stein vor Beiten Den Merlen gleid) ges [thákget (tefte Boetio.) Es follen Die rechten in Enpern viel gefunden wer: Den. 2luch bifweilen in? Torwegen/wie Olaus 230 orm pag. 55. fotreibet, Mran Ean auch Papier Daraus macben/Dafi. man oarauff fofreibet. Luno menur man Die Edrrifft toieber Davon baben soill wirfft mans nur ins Seuer/ po verbsen net Die Sctrifft / uno das झapier ift wieber fautber und rein. 23 ir haben von foldhem Papier nuch ein Stúcflein. Die Utrache/ warumb ber Etoff/und was Daraus gemachet mirb im fetter nicht verbrenne/gibe Kircherus 1.8. fec. 3* mundi fubterr. nemlid / Daß $\mathrm{CB}$ eine Vifcofe ober fohleimichte uno ólidfte Sache bey fich) haberfo nidyt oun ber inz Difhen materia, welche rein und re corruptibili non mixta fónne abgefout Dert werben / gleid) mie eftiche andere mineralia audj folche Eugenden an fich haben/als Das (3jolo uno Das Talcum ober Marien (3lap/weldebes auch burchs F Feur nicht berringert wiro/ twiervol es hely einem fefter als̆ bey bem andern anfalt / Dann in bem Talco nird endlich Die Fettigfeteit in Dem Feur verşbrect/Da Der Amiantus aber uns bas bsolo nimmermefy / wenn es aud) ein ganfs Jabr im Feut lege. Ssibt Daher Kircherus d. I. Diefé Stein's Szefobreis bung alio: Asbeftus lapis eft fibrofus, alumini fchifto haud abfimilis lenta \& eraffa vifciditate, feu lenta \& vifcida craffitie conftans ob omnium partium homogeneum contextum in vaporem refolvi nefcius, folus $a b$ omnium actuofisfima ignis natura immunis \& incombuftibilis, Dafs 
aber aus biefem unverbrennlichem Dachete ober einer andern materia man einimner brennent sicht machen fómme/wie viel autef bon ben alten bars

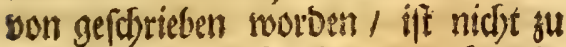
gláuben/ wie ç Kircherus aluch weits Idufftig niberleget unb ausfüfretet.

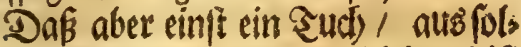
derm Steine gemachet / gleids mol ift berbranot woroen / foll burch ein miracul geforefhen feun / und erseblet Kircherus die Shiftoria ex vita S. Georgii alfo. 2્Al's ber heiligge Georgius ift zum Seur verbammet nourben 1 haben Die Syrannen feinen $\{$ eib in ein fold un serbrenntich Tudb gemicfelt uno ins Feur getworffen / bie CEbriften Darmit su fchimpffen / wennel berbranot twits De/ uno Dab Such, Gliebe ganks / fie jagen Fontew/ ein folcher beiliger \$nann bätte nicht Eónnen ein Miracul thunfitich vom Seur unver fêtret behalten. 2Alber (jott

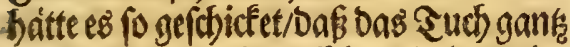
verbrandet / an Dem Corper aber wáre nicht Das geringlfe verfelyet worben. 2Sare siel menns twabr wáre. 230 n biefem Steine baben auds gefdrieben Plinius, Elianus, Solinus, Cardanus, Scaliger, Ludov, Vives, Aldrovandus, Agricola Gesnerus, Sennertuss Licetus unb onvere mehr.

Num.3. Ein Stein/weldhen Ges. nerus Cochleam einen \&effel nennet/ uno mirb vom Comment, de figuris lapid, fol. 164, bef(brieben/ wie aud von Johan de Laet lib.2, de lapidib. aus Dem Gesnero pag. 180. Jftallibes farbe/ hatt/ une bat einer 216farą an ben andern.

Num. 4. Trsie auds 13+ (enno Dons nerfeill. Daf fie aber/ wie man faget/ in ber sufft follen generiret uno imt Donner herunter gefichlagen mertoen/ift nicbt wol zu gláuben / glnublicher aber/ Dafint Donnerwetter eine furphurifose voer fojmeblicjete und falpetrif fore materia in Der \&ufft sufantmen getriteben unb berunter in bie Eroe gefólagen werbe/ uno wo egeinen bequemen Sano antriffit / fo formiret und fogmetset es Darinnen fotche Steine. 2Bie ešaber zugelfe / Dafifie als Seile formiret werts Den/ uno fódher Darein beteminen / ift ungenviffe/ uno mus manes barmit bes antwoorten : quantum eft quod nefcimus. Die Đrobe / of fie yon bee

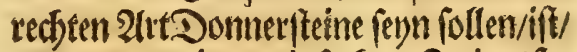
wenn man einen cinfachen Bwimbfas Den umbher bimbet / and auff gliende Foblen leget / fo fort Der Fadennidat betbremen / fonbern nter nafi Dabon werben. Olaus280rm inMufxo p.75.

Num. 5. Ein langer Stein Belemnites auch Dactilus Idreus genant/weil er als eine Srike vom Yfeil / oder noie ein Finger geftalt/ettliche ferno hoht/ets liche boll/etliche flar/etlidse DuncEet/etlio che weich / ettidse fyart'. Man foll fie in Đreuffen und Pommern/unb audb bey șildesbeim finden. Boetius pag. 477 . 


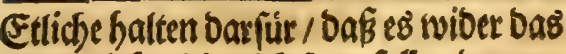
2lpsorticfen bienlido fenn roll / Darbon Olaus Worm in Mufro pag. $7 \mathrm{I}$. weitthumfitiger su tepent.

Num. 6.7.8. unt 12. Senno num Derliche Steine / to unterfobieoliche Jamen haben/ Umbilicus Maris, Ve-

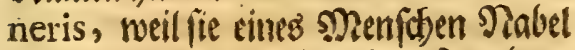
ebnlid) fehen / fonten eine Areerbone genant / fie feynd albier nach iffeer rects ten Giróffe geseichnet. Eso meynen ets

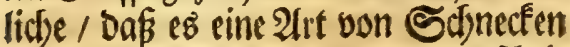

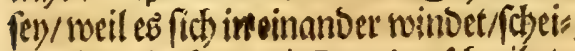

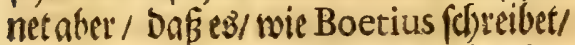
ein Dectel von einer Edjnecten fey/ welcher zur 2Sintergsect am 2)sunolos d)e Der Schnecten gefunden/ uno gegen Den Sommer mieder abgeftoffen wird als man aud an unfern gemeinen Sdfuecten / bie man iffet / fiehet / nur Daps Diefe feftr bicke / jene aber gar buinne fenno. Die Éreinen feynd auff Der $\mathfrak{O}$. berfeiten roth und erhoben/ auff ber Lly terfeite aber plat uno braunroth 1 Derer wir gar viel baben von Venetien bes Fommen. Die groffen/fo aus Jnoiel Eomunen/ fennd auff ber D berfite gants weís. Der Eleinen follen viel am Strant: De Des Şadriatifoten Mreeres gefunben werben.

Man forteibet Diefem Etrin biel Srafft uno 23ircfung zu / unt traite biel/ wenn ešnur halb waftr. Eir foll Denen fo ifnantrigen / zu Den 2lugen gut feyn/Daber ibn bie Eretemer nicyst
Umbilicum, fonoern Oculum maris nennen. Won Der. Venus aber bes Fommter Den Namen / quia à puellis geftatus gratiam conciliare, illas amabiles pulchras virisq́ue optatas reddere, illarumque morbos abigere creditur. Er foll aucl) / menn er ges tragen wiro/ yor bie siofe betwabren. Wä Baccius lib, de gem. \& lapid. natura cap. 40. von bieferm Stein be ridstet ift roarbaffitio Dafi er nemulidb eis ne Fonderlide) Siraffit habe Das Silut zu fillen / wenn er an Der platten Seite mit Speichel beftrichen an Die Stirn getlebet / oder auch auff bie Đulb gebun: Den wird / weldses ids auds bey etlidsen in Der Đirobe gut hefunben. Es berichs tet Boetius lib. 177. Daß Die Syollanbee auds etliche mabl folches probiret. Uno Pey einft ein alt 23 eib / in Dem fie bon Der \&eiter gefallen/ann sopffe verwuns

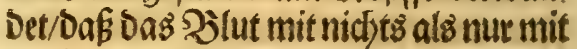
applicirung biefes Strinz babe tonnen geftillet wezoen / weldjes Den Zufehern groffe 2ertwumberung gegeben. Dos gebachter Autor fefet fermet: A Gallis fecreti loco mulieribus nimio menfium fluore laborantibus in pulverem tenuisfimum redactum cum aqua appropriata vel conferva aliqua utilisfimè exhiberi. 2(uch $D_{C}$ nen Eangerwiffe gefolffen merben / bie bisiveilen Bllut fpeven / wernn fie bas Pulver mit gebrantot Sairfogforn ana in einem weidben Ey einnefrmen. 
Num. 8. Terra figillata, baben wir bon unterfochieblicjen Sorten uno Fars

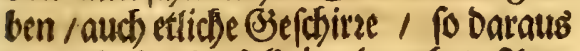
gemadht penno / foll eine berwebte 2 artes ney) wiber Bsiff uno bie rothe Siuhe/ swie aud) miber viel andere STrancefheiten Dienlich pern / worboun Olaus $2530 t m$ unb anbere Medici ausfififrlica.

Num.9. Aftroites, vel lapis ftellaris, Stermitein ober Șiegftein / weil fie meynen, Dấ / mer cinen foldhen Stein bey fids tríget / fod feinem 23iderpart überwinben. Diefer Stein wirb Das ber Aitroites Sternftein genant / weil er boller Stern ift / fie follen in Syrol gefunben neeroen / Elein uno groffe. Marfilius Ficinus nennet Diefen Stein Draconitem, weil/ wie man ifm (bod) fal(d) ) beridstet hat 1 im Drachens ropffe poll gefunben neerben lib.3. c.14. 2lber wir haben fie inßjróffe eines nez Sopffis / soie Boetius fie techt bee

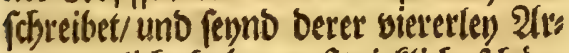
ten / etliche faben austruicllides foróne Sterne/etlidse Siofen/etliche gants buns cfele Siguten/ettiche als 2 Saffertwogen Doer wie 2Bưrme fid Eruimmenbe ?As Dern / wie bie Frguren in folgenber $\mathfrak{T} a$ fel anseiget. 20 allerley. 2Reten ba benwir ettichye. Boetius berichtet aus Dem Cardano lib.7. de fubtilitate, Dem aud) Wormius benfált / Dafis/ roenn Diefer Stein in Effitig gelegetwiro/ fic) hin uno nieber betwegen foll / alb wenn crlebenoig waire/ ich hate eş stwai mit aflerley Alrten verfucteet / aber Eeine fonberliche $\mathfrak{b e m e g u n g}$ fpuren fönnew/ obne in Der erfen 2let / ba bie Sterne ihre Stralen gleid als burchgethers djen und erthaben füfren / fellbige 2lrt

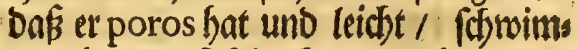
met/ beweeget fich boch gar wenig/andere aber fonderlith Die obfcure fichnarks lichte Siguren haten / fo gemeiniglid in Form eines Şer

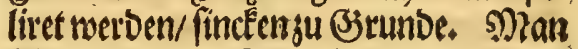
forreiber Diefem Sternftein grofiesirafit uns 2 SBircfung gu / wenn er felbige nut ballb hat/ ifter viel (J)eloes mertf. 2 sill etliche aus ben Autoribus biefer fes Gen. Der Stein pulverifiret uno vier Giran in Darju Dienlichem $23 a f f e r$ ein: genommen / fol ein gut prafervativ Feun wiber bie Mefte. Stem die OYSurs me aus bem \&eibe qu treiben. Estlicte geven vor/oap po mandiefen Stein am Jalfe trage / er Die generation Der ogsurme verfinbern fou. Stem/ miber biefdywere S?oth uno andern gefdhwin Den Firandffleitent. EEtlidge mennen/ noemm fie biejen Stein bey fich tragen/ follen fie ilyren Seind úberwinden Eons nen / Dabet er audb Siegftein genant wiro. Es mag aber etwan einet einen folchen Stein bey fidd gethabt baten/ uns hat feinen Feind uifertualltiget / to mus es Der Stein verurfachet baben. Diefes Eteins Wufber forl aud \&eber uno \&unge curiren. $238 n m$ man ben s

Strin 
Strin in ein Siemact banget / foll er berbinbern / Daß Eeine Spinne nods giffitg Stbier binein fomme. Olaus Worm bat Diefer mit dem fleinen Stei ne verfuchet uno unrichtig befunden/ was aber einer als ein Siopf grof thun soiro/ftehet zu verfuchen. Die Eleinen/ fo als ein Nagel am Jinger gro $8 / w e n n$ Die Sterne fobón / fan er feiner 2 Surs Digkeit balber / Daj man ibn im Singe trage wol 1. oDer 2. Shaler gelten / wie Boetius mernet lib.2, c.147.

Sonft ift nodh eine andere $\mathfrak{A}$ rt Sternitein/Der gar groffe Stern als bie Erbfen bat / fo ein neben Den anbern lang ourds Den Stein gehen , ifor Umb. fang ift weip / hart als ein Riefelftein/ umbber aber ein weicher Jorofer Stein.

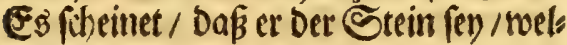
therl Gesnerus uno aus ifm Boetius bejofreibet / und nennen ifn Afteriam uno aud) Lapidem Stellarem. WBie Boetius Denfelben begeictinet/ hat er nut 5. Doppelte Siralen/unfer aber wol 20. fubtile einfache Stralen.

Olaus 230 orm befobreibet aud faft bergleichen Etein/weldher ju Siopen. bagen Anno 1649. vor Der 9?orber: W forte ift aufgegraben morden / foll nur 8. Stern uno Die Figur fünfectet feyn. 2rsir aber baben zroen folcher Steine / Deren eine -gróffer als ein Manns:Sopffuber 200. Sternen/ Der ander etwas Eleiner / fo benbe Alichefars

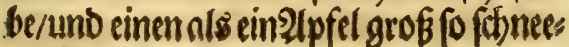
meif.
Num. Ir. Aëtites ein 210lerftein/ befommt ben Namen vom Abler / weil er in Des Polers Nepte unter feinen Evern foll gefunden werden / unb wiro bon ibm binein getragen/ entwoeder Dapi weil Der 2loler in Der 23rute alljubizzig/ Der Stein Dass temperament geten/ ober bie 2lubbritung befordern foll. Boetius befchreiber ibn ausfubrlich/ uni gibe berfelben orey Species, bon benen wir groen faben runde als 28 alls múfegro $\bar{B}$ / fo fobwarkfabl/ und einen groffer als ein Scuner:Ev) / aber etwar platter uno gellid)t / Die erften renno Oriental, bie anbere gelber 2irt/Gxodes genant/ wirt in Deutjphlano snois iden Drefsen uno (Shemnts aud in Sobmen bey Dillen im 26affer gefuns Den / alle von auffen grobangugreiffen/ baben invenoia noch einen Eleinen Stein/Denfie Callimum nennen : etlis d)e etrons כobn ober farte ErDe / uno Hlappern alle / Daber fie aud Slappers feine genant roetoen. Die in Africa fallen/ ferno Elein/haten weiche Erove in (iid)/uno werben 2 seibliches/Die aber in Arabia fallen/fenno bart/baben Steine in fich / und werben \$Nänuliches (5jes (chled)tes gebalten.

Man forreibet Diefem Stein úter als le maffe beraliche Sugend zu / uno wenn er foldye barthut / ift er viel (B)eloes werth/wie Denn ber experientz Der fury nebmen gelehrten Medicorum, Die fols đjes betraffrigen/. S3lauben zuzultellen. 2ity 
2Ben Den Sod fte Plinio \& Galeno; Die Sirafft Die Frucht nach fich zu jieben / uno Die (Sies burt ju befooern. Darumb die Proclives ad abortum fento ob uteri lubricitatem, Die follen ifn an Den lincfen 2trm binben. Bur Beit Der Seburt an Die lincte Suffite / fo foll eB bie (sicburt gewaltig befóroern / weldbeb Olaus EBorm / Der weylano furtrefflid)e bes uifmte \&eib. Medicus ģbr. Són. . 2ai. fu Dennemard in Sopenbagen etlidse mabl in Der jrobe gut befunben (vide Mufxum ipfius pag 78.) Die anoere 2Itt alı Geodes foll in defperaten Fallen bey vornebmen Dratronen auch guten effect gethan faben. ibid.

Aber Das if Darbey in actyt zu nebs men / Das fo balo bie Frau erlofet uno bie Sischurt gefchefien / mus man ben Stein wieber abnehmen / fonlt foll er matricem mit heraus ziefen: $23 i e$ Damu / referente Franc. Valleriola, einft in Valentia geichefjen / Dapeine bornebme Frau burch foldhes Strins Sculffe eine leidte (jeburt gebabt / weil man aber ben Etein an Der Scuffte ber: gefien/ift matrix mit herraus gefdyofs pen/Dap fie Daruber Des Todes feyn múf fen. Dietodte Bieburt aber foll es nicht treiben/wie man mir gefaget.

Diofcorides gebendet nod einer ans Dern Sugeno/Daker nemlich einenDieb verratben foll. 2Senn manetwas bon Deffellen Steins Julber im Broo eir. nem Werbáchtigen fu effen gibt / fo er fifulbig ift/fan er unmuglich bas Rirod einfdhlucfen. Bellonius fbreibet audy) Daß Die (S)riechen Munche Diefen Stein Definegen bod) balten / Dann wenn fie was verlobren/ruffen fie alle in Silofter jufammen / uno geten im \$rodte jeglis d)em Drey Munbbiffen ju effery/mit etlis d)en aberglaubifoben zugefasten 230 v. terw/po foll ber Dicb im lekgten Siffew Den er nicht binter bringen Ean / fich vers rathen. Der Stein Eteinguftoffen mit Corato, ober gehl Salbier Zugpflafter bermifchet/ úber bie Stitn uno Schlaff geleget forl bie binfallende Sucts lin bern uno bertreiben / weldbes 230 rm nicft miberfprecben mill. Der Seim/ Shon ober Erbe/ fo im Stein Geode ift / wenn fie gewafichen uno mit 2 Bein gutrinden gegeben witb / foll fie ઝefte unb andere gifftige Sirandtheit bertreis

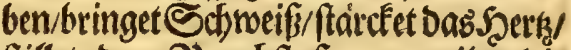

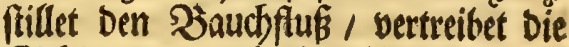
Schlangen / umb Diefer Urfach willen mennen etlidbe / trage ifon ber 2 bler in fein Pleft. Dak aber etliche mennen/mer biefen Stein bey fich traiget / foll gelies bet/und reich werben/wirb fúr aberglaius bifs uno fabelbafft gehalten.

\section{TABULA XXXIV.}

Num.1 +3. uno 5. 2Berben auds une ter Die Aftroitides geredynet/uno fenno in oer vorigen Tabelle bey num. 9. bes gii

㑗rite 
pdrieben worben. 280 bet aber for de Sterne und anbern formen in fols dese Steine fommen/if mit mebren in Kircheri mundo fubter: lib.8. $3 \mathrm{u}$ lefen/ nuirbe mir aber fier/Der ids mir nur ber Siúrşe befleiflige/ mit cinjufübren zu weitláuftig fallen.

Num.2. Fungites if ein Stein rinem Sdymain / fo aus ber Erben waidspet/gar efnnlich / als wenn er ein Stbmam genvefen und zuStein getwot: Den/hat aber Eein Stiel ooer 2injeigung Daßj cin Stiel Daran getwe én/Clur.lib. 6. Exot.c.ro mennet Daß fie theils im Inoianifchen Nheer / theils im Nilo wachjen folten. Num. 4. aber ber audh ein Schnam fich gleidhet / bat uns ten einen $\mathscr{2} 6\left[a a_{g} / \mathrm{D} a \tilde{\beta}\right.$ man vermeinen folterer boitte einen Suf gethabt. Uno if biefer andez Schneetwei í/uno gar fádón formiret. Es sift aber nicht su vermuthen/ Daßjes Fungi over Schnamme/fo aus Der Erven machfe / getwefen feund. Dann bie Natur (pielet wumberlich) I Es Ean einer Der bie Beefhópffe Der Dingeffo in ber Eroen / uno fonderlich unter ben Steinen gefunden werden/ mit emfigen uno Elugen 2 (ugen anfehen wil/fich nicht gnugfam bestwunbern/wie ingeniofe bie Natur fpielet/afíp / Daß was fie in regno vegetabilium \& animalium verrichtet/gleich)(am auch in benSteinemnach iherer 2 rt intendiret, und po viel ifer vergónt und fie getuno Dabin gearbeitet/Dapi welchen fie Das̆
Seben und $\mathfrak{X}$ enregung (infimo illo repugnante Naturæ gradu ) nidjtges ben fơmen / fie bod Den Steinen nut bie figur einorudfen toollen. Sa bas nod) mebr iff; Sie láfet audb ibre Bes liebung gu Den freven Sinften in etlis cben EDDeln und anbern Steinen bers mercfev/indem/mas in ber Geometria an Tiguren vorgebet/fie auch præfent:ret als : Triangel/ Duabrate/ s. uno meble eficht nicht nur im platte fondern aut in folidis corporibus, nad) bet trinam dimenfionem Sphrerifohe/Cylindrifobe/Comifote / Pyramidalifobes Cubifche/ uub andere Eorper/ wie Ders felben Kircherus viel uno auch theils wir baben / unter andern baben wir 3. metallifthe Corpora als bie gemeis nen 28 úrffel gro 3 / fo jeglicher adjt ius fte gleich feitige sriangel an fich bat 1 zwey feuno fiswarkgrun / uno eines Solofarbe / fither / laffen fich Doch ficneiden/faft wie talcum jeboch etroas bairter/feuno vor wenig Jabren von Dem WBolgebohrnen Scerrn $\mathcal{C}$ laub $\mathcal{C}$ Griftoff

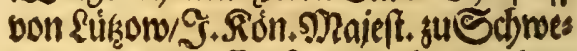
ben scerr Scoffs s)arfchall aus ben Schwedichen Begwercfen mir zuges foticket/follen alfo zmifoben Steinen ges funben feen. In ber Architectur ift bie Natur auch gefdoaffig / uno gibe Sacufer / Sablófer uns Stábte/wie auth im (Sartenbau (c) one Sáame uno Bufdwercf. 2Bie benn folde Steis ne in Stalien biel fallen. Huter anbern baben 

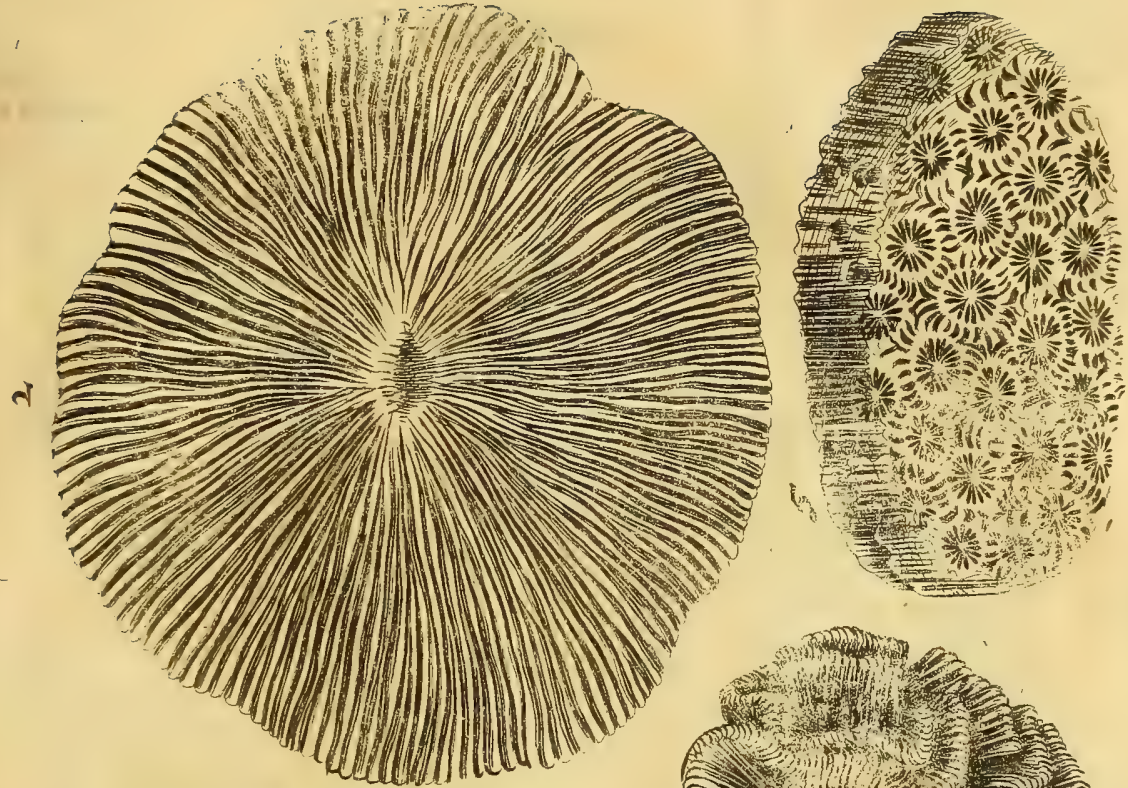

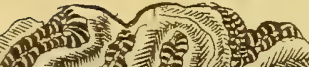

(

-

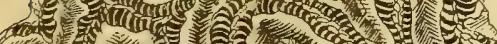

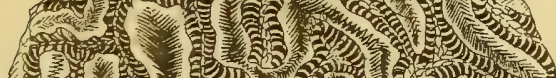

- 4mon 175).

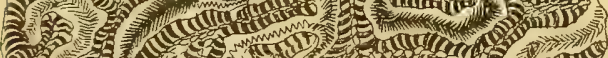

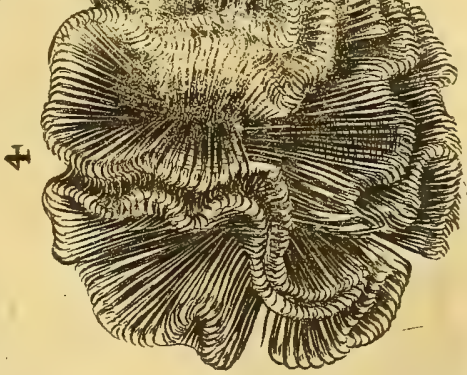
จ. จ P.

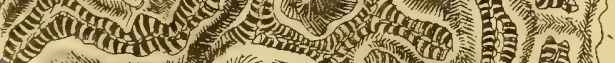
56. 3.

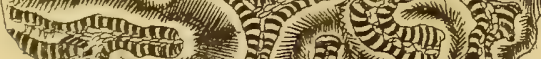
4ugen F.

$\stackrel{2}{2}$

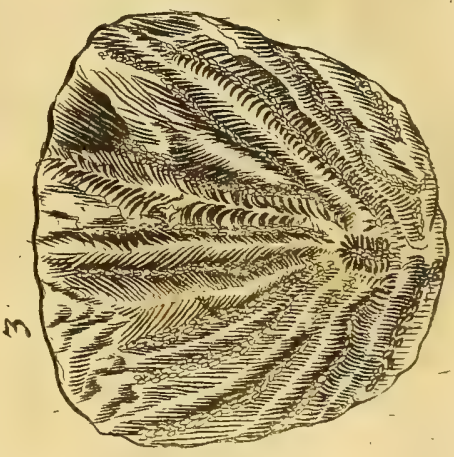





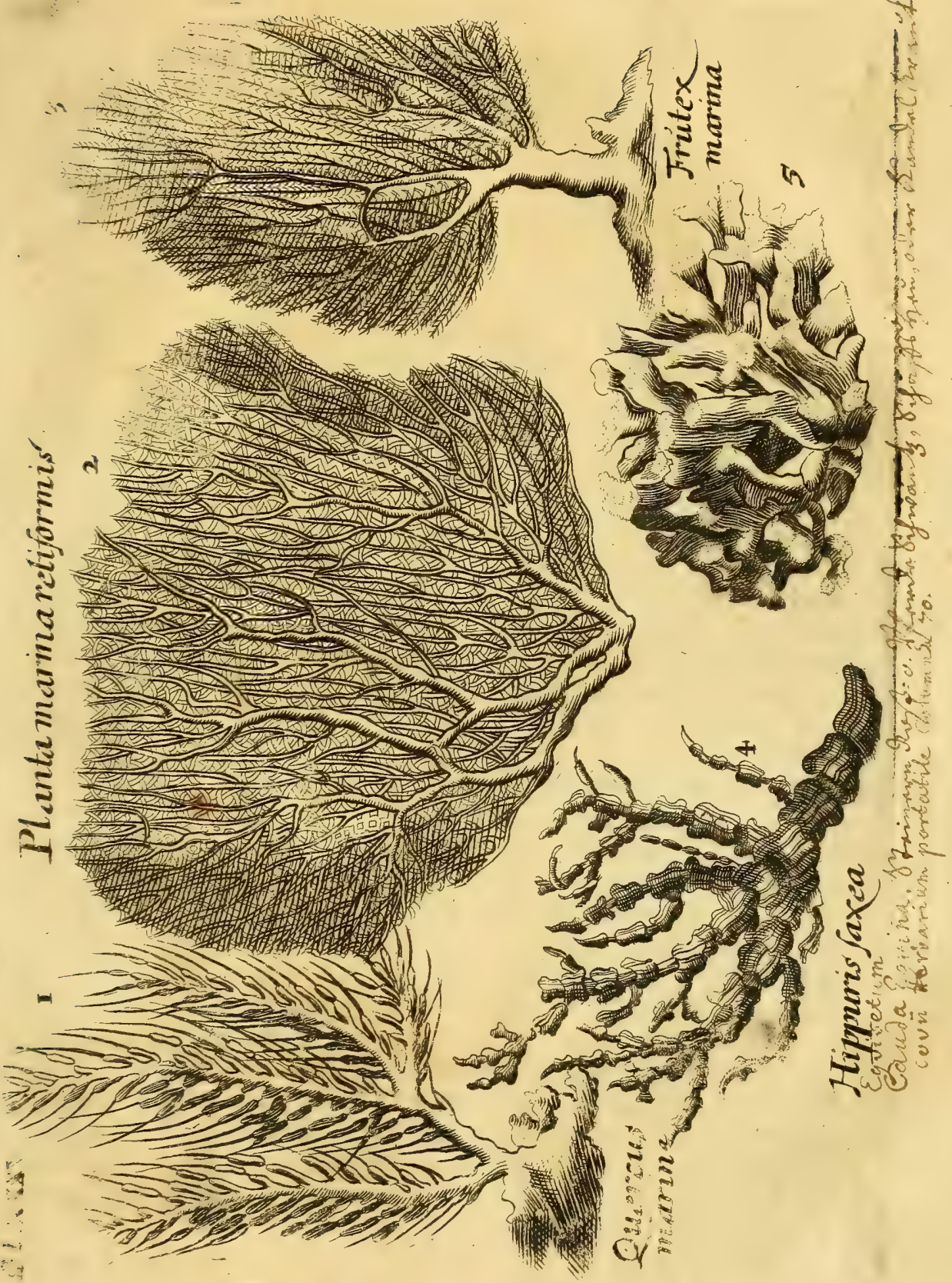



baben wir sweene fletine 2 gaten / welche \$fr. Churf.Durchl.ju Srandenburg (fo ein groffer \&ieb baber ber rarennas turrlichen wie aud Eünftlichen Dingen) als fie vor 6. Jahrenallbier auff Sots torff in unfer Sunfte Sammer gemeferty binein verebret/in beren einem ein fchón Süfb/über welchen ein \&ơme (pringet/ uns im anbern ein fobón roft Saáums leimfo bende lieblich ansuferen. Natura fompt auch zur Aftronomia, mas chet Stern / Sonn und 9)iond. 21uch anbere Dinge mehr in Eirófe unt Proportion Der lehendigen Eleaturen/ Daß man meynen folte/fie wáren aus Den les benoigen Dingen zu Stein gewoorben.

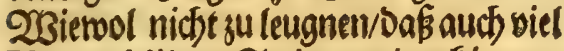
Vegetabilia zuSteine weroen fúnnen/ mie Die Exemper betand feyno/ und wir aud) baben.

\section{TABULA XXXV.}

Tn biefer Tabel fenno lauter Sees Eserwächfeberer wir untáblict biet bas ben / you allertey 2fert / feyno hart als Scols boer vieltmeht Scout / laffen fich auch alfo fóneiben uno beugen/etliche

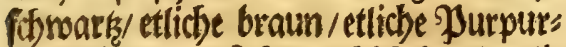
farbe Elar und faft Durd)/breineno/etlis We eine Santivetlidfe auch 2. Fus lang/ swachien im Mreer auff Steinem / wie Dann an vielen nod) Die Steine geblies ben/ und baben eine Saaut/ fo yon Den SJerwádjên ab úber fiegebetals seber anjugreiffen. Num. I. wirb quercus marina bon Theophrafto Erefio lib. 4. cap. 7. genañt/uno Dafelbft woie auds vom Clufio Exotic, lib. 6. cap. 4, bes forrieben.

Num, 1. 2. uno 3. Plantæe marinæe retiformes fenno als ein Nefs ineinan Der geroads (en/wirb aud) beum Clufio d. 1 . bejeichnet und befobrieben. Diefe beyberley Meer: Beswaichle baben bie Art/Dap fie ibre Znorige nicbt runb umb fiid ber / fondern nur plat ausgieffen / Daß man alle zugleich auff ein Sitet les gen uno ausbreiten fan.

Num, 4. Diefe Neerpflanke wirt Hippuris faxea genant/ feht fdjon unb lieblich anjufeben. Der Stengel uno

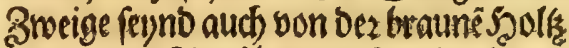

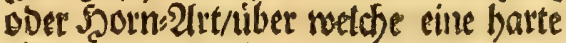
Stein materia fich geferet/als nie weif fe geftreiffte Corallen / fo weit bon ein ander/ Dap man als ein par Meffertus

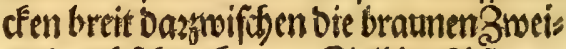
ge Durch fefen tan. Selbige Pylanke wirb auch bom Clufio in Exot. lib, 6. c. 8. bejeichnet und befdrieben.

Num. 5. Sit ein Stam bon weif fen Evorallen/Derer wir etliche und einen son so. Dy funden baben. Sonft haben wir auch fichone groffe Zneige bon robs ten (Sorallen/ tmo etlicbe / fo nod) ifre Mutter/woraus fie errwachien/ an fich baben/roie aud einen fobwarken Eorals lem: Stamm.

$$
\text { giii TA. }
$$




\section{T ABULA XXXVI.}

Num. I. Sftein (Jlą Urna lacrymarum genannt/ foft ein $23 i e r t e l$ einer Ellen lang / im welche die Saenden ibre Shzanen/o fie megen ifrer berftozbenen Freunde vergofien / gefamlet/und her: nach Des verbranoten Eórpers 2(ch) Damit befeudftet/und alfo begraben/wie Darbon Kirchmannus de funeribus Romanoruum lib.3. cap . 8. Man fins Det auds in ben Sensoniffsen $\mathfrak{B}$ egrábs niffen foldbe Ssáfer.

Num. 2. Sit eine Sampe / welctse man aud bey ben Saenonifoben 2 bes grábniffen gebraucbet/auch Darin gefuns Den werden. Do aber das lumen perpetuum Darin folte haben erhalten wers Den Eonnen ift nicht wol gu gláuben/wels ches audy/mie of $\operatorname{ced}$ ad)t/Kircherus in mundo fubterran : wiber (prrictt. Man mennet aber / Dấ / weil man nur ben Practece bießeichen begzaben múffen/(noie. Alex ab Alex, 1.3. c.7. (aget) hat man

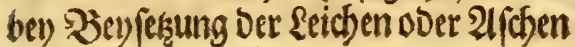
gebraud)et.

Num.3. Jif eine Urna ober Sopff/ in welchem Die Szenden bic 2liche uno sibergebliebene Stucten Sinothen Der verbrandetr tooteresézper gefamlet uno zum Begrábnif bengefestet. Selbige batman Cineraria aucb Offuaria ges

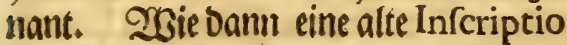
(referente Kirchmanno ) gefunoen worben/mit biefen 230 otten: JULIA.
FUSCINIA, OSSUARIUIM. VIVA. SIBI. FECIT. Und fenno folde Utrnæ nad) Standes und condition Der Menjchen von Bsold/ Sillber/Etr/ Stein und Stbon gejegrtworden. 233 ie Denndes Säyfers Stajani arche in eis

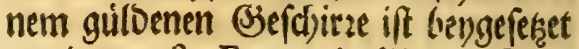
noorden/tefte Eutropio lib.8. Won Der fillhern Urna beridbtet Am.Marcellinus lib. 19. Won Denen poaug Eres aber Virg. Æned.6.

Offaq; lecta cado texit chorineus ahéno.

Etine Urnam von Porphir Stein hat i,m Ránfer Severus erwelklet. Dann man faget/ Dak als er furs vor feinem Fobe felbige Urnam vor fid) bringen laffen/ in bie Sano genommen / uno gee faget : Tu virum capies, quem totus orbis terrarum non cepit. Du wirlt Den Mnann in Dir nefmen/melchen Die ganģe $25 e$ elt nidft úberwältigen fón nen. Dieferlbige Urna, fo wir haben ift von S bon/und Anno 1649; aus ber Nider\{auknis/ bon einem vornefmen Jebienten am Effurfiriticiden Szofe zu Sachien/ Nabmens (Safper von $3 a$

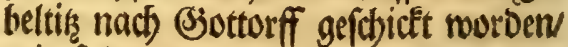
mit folgenbem Serericht: Diefe bies fchirze Eönnen nidjt ehe als umb æy fings fen aufgegraben weroen. Uno vers bailt fich mit Denfelben alfo / Dap fiefich umb Diefe Zeit in etwas heraus beges ben/ ober aus Der Erden bobber herauff ferigen uno niche fo tieffi in ber Eroen 


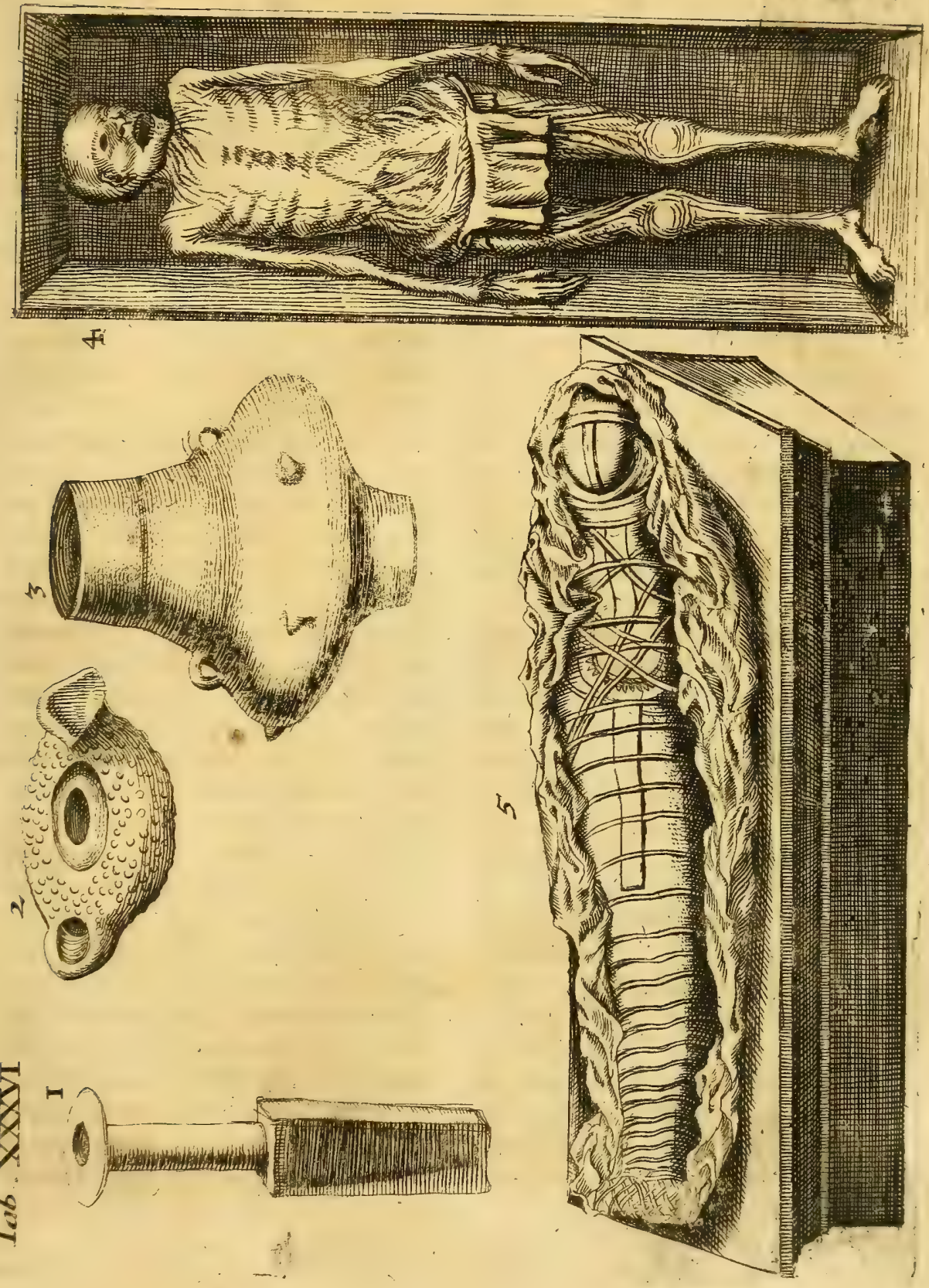



fteben follen / wie in gemein barbor ges balter niro/ uno wenn man fie aufgeris bet / ift bie materia gans múrbe uno mufi gar fubtil Darmit umbgangen wers Den/ Da ja manfie gan berauf befompt/ wenn fie aber nur ein wenig an ber \&uffi geftanden und tructen worben / nefimen fie erft eine Sairte an fich. Etliche

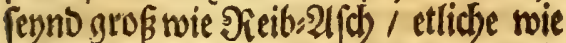

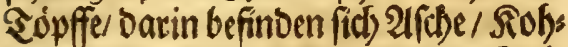
len uno Sinochen wie fleine Rinder:Seis ne/auch jumeilen Stuctlein झूetall wie farck Drat/ fo mebrentheils Supffer/ uno fich wie (Solo arbeiten láfet. Etti: che Eeute balten aberglnubich Darbor/ wenn man aus felbigen (Siefchirzen bie (3)artens Saainen fäet / follen fie fruchts bat werben. Sie baben fonft meines Erachtens Das Anjehen / als wenn es Seionifche Begrabuiffen wairen / weiln

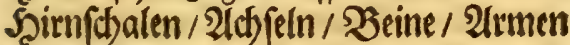
uno SeinEndobel nebent: Soblen und 21foben barinnen gefunden werben.

Num. 4. Ift ein auffgeburzeter Ins Dianer/weliffer ben \&eib nod) gans und feine (S)ebarme in fich hat. Selbigen haben mir auch mit aus Des Paludani Sunft: Fammer befommen / uno wirb auch feiner bom Camerario in horis fuccifivis cap. 70. Der andern Centu-

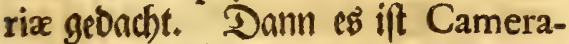
rius in Zweiffel geftanden / ohs wabs fey/mas man voul benOccidentalifchen Inbianern fobreibet / Dap Dafelteft auff Den bodjften Sergen su gemiffen Bei ten DeE Sabres \&eute toot / roelde am Seibe unverfebret/ mit Fingeweibe uno allen SSliebern erftartet / uno geburzet gefunoen merben / bif ifn D. Paludanus ber furtrefftiche Medicus in $\mathrm{Scol}$ lano rerum exoticarum utriusć; Indiæ perfcrutator \& collector ftudiofisfimus berichtet / Dafs er zweene foldhe ausgetructnete s)enfdren in feinent Saufe hatte/ bie gank Eein (Sieruch bon fich gebennuno boch oon ferne antureben mairen/ als wenn fie lebeten. Zun bies fen beyoen/ift Der eine fo wir baben / as ber nunmebr ganes gefl uno einges fobrumpen/tlinget als eime Ylaucte / fo nidbt fteiff angejogen ift / wenn man auff Den feib foblaget. Die Utfad) foldser ertóbteten uno außsgeorógeten Sórper gitb Camerarius aus Corneli. Witflieti notitiâ Occidentis, Daß in Der Sanb/chaffit Chili auff Den bohen Gies bitgen ein foldber rsins jumeilen ents

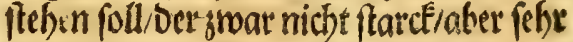
fultil und Durchoringent / Dafs er intima prac ordia Dutch bringe / beriebme Die natúrliche 2 Barme / erfticle bie Ses

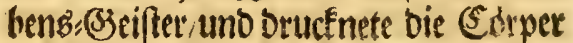
alsbalo Durd uno burd) aus/Damit Eeis ne corruption uno Faulung / fis bon WBaime uno Feudtigkeit berathret/ fatt baben tan.

Die Spanier haben zwar in Den ers fen Jabren / nacl) Dem bif Sónigreich som Almagro auffaefuchet worben ibs ren 2 Seg aus Yeru úber \&ano Dabin ges

nommen/ 
nommen / - meil aber foldse gefáfrlidge rainbe ben Sieifenten alfo fóáblidy/ Das theils ibr Seben/theils Die Bebe bon Den Fúlen unbermerctet verlobren / ba ben fie fid betnad Der Scbiffabrt bow bingebraudjet. Fs erjeblet jekt erwebn ter W W ithiet, roie aud Jofephus de Acolta in feiner 2 bef Snbifbenspiftos ria lib.3. cap. 9. Sollánbif́ches Sru

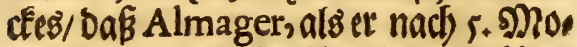
nat wieber zurucfe ubet Das SSebirge Fommen/siel Der Seinigen / fo ibm ents gegen fommen wollen/mit Den Oj ferben toot erftarset/uno feiff in oer Dronung/ wie fie geritten/ febenb angetroffen / fo noch bie Pferbestigel in sainoen ges babt.

Sonften findet man bergleidoen aup; gebrógete Menfoben in einer africanis fón 23 úften úber DenNilum imsan beftecten. Dann weñ ein fatcfel' 2 Sinto entftebet/fol er ben leidjten Sand/bels कet Dafelbft bauffig/sufammen treiben baв eś als ein Sants)zeer módte ges nant wetoen/in welcbem die seute er|tis cen / uno bon bet Gonnen Shife uno beiffent Canbe gants aubgeburret wer: ben. Gn foldsem Sanos) neer foll bes Cambyfis Rơnigs in Jerfien faft gan seb Seet umb Eommen feyn/ wie Herodotus in Thalia forreibet/ aus $\mathfrak{B e r i d}$ t Det Ammonier, weldbe Cambn fes bat betriegen mollen / Dann inbem fie aus Det Stadt Oafi ihten 23 ge Dutch Den Sano auf bie Ammonier fugenoms men/ uno mitten im Sanofeloe Mnits

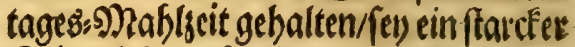
Subroino entfanden / Det fie in Den. Sano: Sneer múffe vergraben baben weil fie nicht fu ben Ammoniern, audb nidjt wieber juricé geÉommen.

Num.5. Ifteine gankge Mumia aus einem vornebmen Egyptifchen 23 grábnis/lieget in einem Siaften/uno gibt einen guten (Setuch/wenn man ben Sa ften aufithut/son fich. Dieben biefer ftes bet aud) ein Sino / als ein Mumia.

Daz 230 rt Mumi ift ein Perfif Wort/uno beiffet ein aufgedóczeter uno

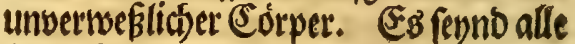
foldbe Art Mumien, umb Die Sjetwes fung su verbuten / in gewiffe Gummi und materien geleget / und mit sti d)ern/ wie Die Rinber in 23 Bndeln ein gewiclét / umbwunden. Sie weroent vom Plinio funera medicata genant. Dann man will oafur balten / Dafifte

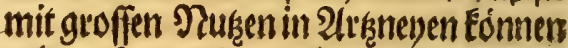
gebrauchet weroen/ wie man oann auds faft in allen wolleptalten 21potecfen etlis che Stuicfe Darwon findet. Francifcus I. Der Francfen Sónig foll allezeit auffere nen Oeifen cine Mumia, gleid) alb eit fpecial medicament mit fich fúfren laffen / tefte Camerario cap. 70. cent,2, 2Senn aber alle Mumix nicjts mefr in fich balten / als Johan: Nardius ingeniofus Medicus in einer ana. tomirten Mumia gefunden hat / nent (ich) nut asphaltum ober Subenpecbst oline 
obne einige preciofe aromatifdse (๖a: d)en/fo weis idh nicht / ob fold condi. mentum fine cadavere ipfo auch fo

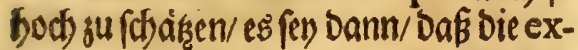
perientz(Medicorum optimum fundamentum) einen fonberlid)en effect gefunben bátte / bielleicht ob mixturam eada veris cum asphalto, Nardii verba funt Comment, in Lucretum. pag. 635. Speravi olim miram fragrantiam à medicinali funere tot tantisque infarto aromatibus, verum fpes me fefellit folaque fuit obvia gummi graveolentia \&c . Item, fcifitor interim curiofus calvariam eodemque gummi intus illitam deprehendo, tunc verò me operam lufiffe omnem libere fum faflus; cum partes cadaveris fingulas attentusexamino altoć; gummi imbibitas video. Fregi, difcerpli,osfibus neq; peperei,gummi ubiq; redolent atq; fapiunt. Serus ego damnavi tandem propriam crudelitatem, longumq; valedixi Egyptiis technis.

PBeil nun an Den Mumien Daš Asphaltum, als an fích felbftein gar bart

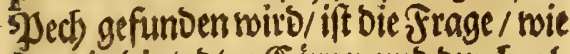
fie es in oie todten (Sorper unb ourch als le Sslieber Dringeno bringen Fonnen. Nardius mevnet / wie auch gláublich/ baj mann Soike zu biefem jectse unb ein Oleum petroleum ober Neffte Eommt / es torid) unb fieffent fan ges

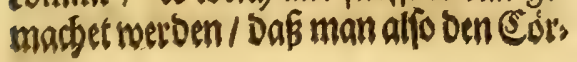

per in ein fold warm $\mathfrak{B a b}$ geleget / fo lange Darinnen maceriret / bis bab Gummi eingegegen. Und Damit Das Beffirne Der incorruption nidft etwa verhinderlich fenn mód)te / baben fie es// wie Herodotus in Euterpe p. 135 . les: richtet/mit einen Erummen Eifen ourd Die Jiafto cber feraus gejogen/tmo atfo Dem Asphalto einen freven Eingang fum Scirn; beitel gemachet : wie wir Dann audf einen balben Sirnfobeitel has Gen/Der gans vomAsphalto gefúllet ift: Den $\mathfrak{B a u c h}$ baben fie mit einem id arffen Ethiopiffben Steine aufforfonitten bas Eingeneibe heraus genommen/mit Walmwein Den \&eib aufgenwafchen/und bernad) nad iber 2 ret bal famiret / mit folchem Sieinigen und andern Eeremos nien follen fie viel sage zugebracht has ben/wie Herodotus an gebaditem Orte weitlaiuftig berichtet.

Sch balte nidbt/ Daf einige Nation unter Der Sonnen fey/Die groffern Fleip angewendet / bie abgelebte ce orper jujies ren/ ju effen/und von ber Fáulung und 2nfto 3 zuberwabren/als bie Ægyptier. 2us was Uthrfachen aber foldbesges fcheben / ferno siel gelebrte feute in ameiffel geftanden. Camerarius loco fupra citato fpricht : an autem $Æ$ gyptii oftentatione quadam vel nimio fuorum amore tantam curam impenderint mortuis, vel alias cau. fas habuerint, aliis excutiendum relinquo. Esiftabergu wiffen/erftlidy \& Dafis 
Dapa bie Egyptier gegláubet / Dấ alle 40. taufeno Sabe bie 20elt in ifren bo: rigen Stano Eommen / und alles was Domablsfict bugetragen/wieberumb ges fobehen foite. 230 oiefen foll Plato, als ber Agyptifchen Brieffer Difcipul, peis ne Meeynung von Der groffen revolutione mundi betommen / unb auffans Dere fortgepflantget haben/ wie Kircherus in Oedipo Ægyptiaco pag. 392. beridfet. 2sietwol mit bem Unter'icheio/ $\mathrm{Da} \hat{\mathrm{a}}$ er bie revolutionem animarum circulo r0000. annorum definiret.

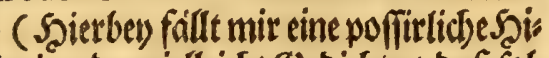
ftorie/ ober viellleidst (S)ebichte / Dap Fole cher Opinion fich einft tweene Studen-

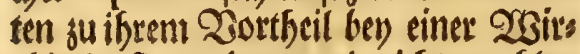
thin/Da fie gefsebret/ uno nicht 3 u jablen gebad) / gebraudsen wollen/ indem fie gefaget menn fie uber ro taufeno Jabren wieber fámen/molten fie bejablen/Daun

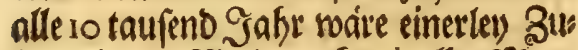
ftanb in Der $2 W_{3}$ elt/ auds mit allengiens fchen/ Da bitte bie 2Sirthin gefraget ; S6 fie baun vor 10. taufeno Sabren auch in foliser befchaffenteit bey ibe gewe fen/und als fie mit'sa geantwortet/ bat fie gefaget; fo werdet ibe eben fo nool Damals nicht bejablet haben/ Derowes

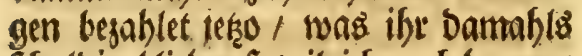
ichulbig bliebenfo wil ich ends borgen. bie die sehen taufeno Jabre wieder uno (enno.)

Bum anbern faben Die Egyptier gegláubet / Daß Die Seels unfterblich! unb follen fie/toie Herod berichtet/uns. ter ben Selyben bie eryten getoefen feyn fo Die Unfterblidfeit bet Seelen ge gláubet/ aber alfo: Quod anima per

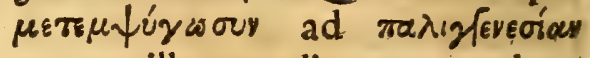
magno illo mundi anno revoluto corporibus primis reftituantur. Daf nemlich Die Seelen Der MRenfchen aus einem verfforbenem \&eibe in ein ander: aus Dem andern in Den britten / uno fo fort an berumb fabren follte / und von neuen Rindern gebohren twerben / bís 7.taufend Sabe verfioffen / fo Eime fie wieder zu ihrem er|ten \&́eib/Der aber mus fte rein und unverfefbret erbalten wers Den/alobann muirbe bie Seele / menn fie wol abgefdieden / fu einer foblern Sphara befodert/ unb uiber 7. taufeno

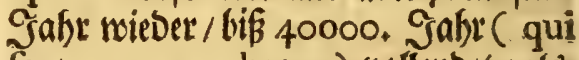
fept.num. quadratus) vollenoet / alb? Dann Étme Die Seele erf zu iffrer reedten enigen Siuhe Da fie nidjt melyr umbjer wandern ourfffte. Und weil fie vermeys

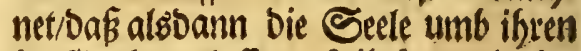
im Sirabe verlaffenen \&eib berumb gins ge/fo múrbe fie Durch bas Urtheil Des Sicchters/10 Darben funde/nicht ba bins ein/fondern in unreine Sthiere boer ges ringere Córper verwiefen merben / gus mabl menn fie etwa nicbt nool gelebet.

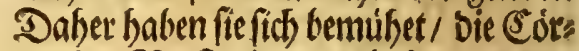
per Der Werftorbenen mit fonberlichen Eeremonien zu efreen / zu jieren uno vove allem 2inftó zu berwabren / audi vor ibrem 2 fffterben felloff fromb ju leben/ Don 
Dap fie bie Seelen bey fo offt veränder" ten 230 obnungen/gut Quartier befom men / uno auds mit sult in bie vorigen Eórper gehen módten. Daher bie reich: flen fo éftlich baffaminet / und wie Die Sinber eingeminbelt (nicht ofhe myfterio iffer neuen (jeburt) uns in fehr Eoftbar gebautet Begrábniffe / unter boch auffgerichtete Pyramides fenno. berleget wortoen. Uno Disift aud bie Urfacte / warumb fie auffifre 230 bn baufer wenig / auff bie Begrábniffen as ber fefre groffe UnEelten getwenbet: bot: gebeno/ man múfte mehe Darauff wens Den / no man fo viel taufeno Sabr inne bleiben fọlte/als auff Dieşuitten/Die man fo Eurgęe \&ebens: Beit gebrauchen Eónte.

9) ?an findet auch an ben sucketn/ in swelche Die Mumien getwicfelte / viel und fellģame Hieroglyphica uno Characte. res gefegtzet / wie Derfellben verfchiedene 2bbillounge im Oedipo Agyptiaco Deb Kircheri, ber von $\mathfrak{B} e$ fchaffenbeit Der Mumien ausfrübrlich) gefdorieben//su fefen. Unfere Mumia bat aud Egypti-

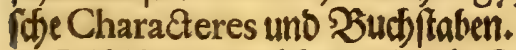

Disift ber Beridst von Den in Supfs. fer geftochenen Tabulen. Sonft ferno noch fehr vielmebr notable Sachen/ fo nicht in biefen Zabeln begriffen: Eơnnen Eunffitig folgen/menn Bistt \&elen/ Bies funobeit und Friede berleiben woird.

\section{2lubang.}

9) Tur ief̧o nod / umb bie leeren Bläts

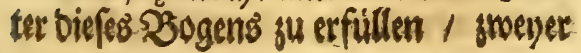

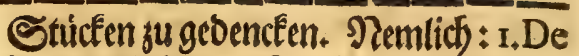
lapide Malaccenfi. Uuno 2, DeSuccino ober ßernftein.

20 ir baben einen fonberlichen Stein/ Der billich/ben) ßefchreibung Deß Bezoars p. Is. baitte follen geovadst werben. Dannmie man Darfúr balt / er Den bes fen Bezoar an Sugeno úbertreffen fou. Ex hat bie Soróffe einer groffen $25 a$ als

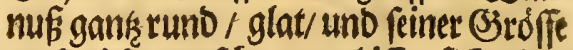
nach nicht gar fobwer / láffet fidd leicht

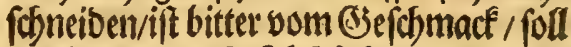
nur in Det PenInful Malacca, fo nicht ferne von ber Aquinoctial lini neheft Der groffen ЭnfulSumatra lieget/fallen/ Daber er audj von Gartias ab Horto, lapis Malaccenfis genant wirb / man foll ifn in ber Baslle eines wilben Sd)weins/ aud) etfliche/aber Eleiner / in Den Stadyel:Sdjweinen finden. Das ber er von etlibjen lapis Porcinus, mie audb bon ben झortugiefen Piedra de Puerco getitulitet wirb. Er foll bon den Indianern in fo boben 28 ert gehalten werben/Daßs er umb gro als ein grof Profent an Den łortugis fif hen Gubernatorn iff verebret wors Den. 2Benn Der groffe Mzogul einen

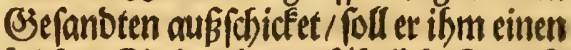
folchen Stein wioer gefábrlichesirances: beit mitgeben. Seine Sugend foul fidy/ mie gebadjet / weit úber Den Bezoar ets Itrecten / wiber alle Siiffit uno gifftige. Rrancéf feiten bienten / wenn man ifn in 23seinleget/und Darbon jutrinden gibt. $\Re$ ii 
Soll Den Shymeis gerwaltig treiben. Der treffliche Medicus Dimas Bosque Valentinus, foll ibn an gwenen פlata nern/ Fo Bsiffi genommen batten / probi: ret haben/ umo befunden / Dafítinnen Der Giift nichts gefchadet / ob Der Stein

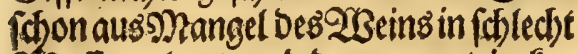
$23 a$ ffer geleget/. und Darvon ju trincken gegeben worden. Jacobus Bontius, if ordinarius Medicus in novaBatavia, gedenclet in feinem Tractatu de medicamentis Indorum, Dẩ er zmeene fols che Steine gefrabt / einen Eleinen aus Dem Stadher Sdbroine / uns einen groffen aus einem 2 silben Schwein/ bat fie fefre gut befuncen / adverfus Choleram fpricft er / quam mordexi Infulani vocant, \& hic tantopere timetur, quam peftis in Hollandia,utpote qua hominem aliquando foleat occidere pauciflimis horis. Prægnantibus tamen hic lapis non bene datur,nam abortum provocare adeo certum eft,ut fermine Malaicæ mihi retulerint,fi quando menftrui earum purgatio non bene procedat, fi faltem hunc lapidem manu geftarent, ad juvamentum inde fentire. Şd) muß biermit gedenten / was er bom Calculo in homine concreto ferner fchrsibet. Profectò mihi confideranti,inquit, judicium humanum de omnibus hifce concretis lapidibus in ventribus ac cavitatibus animalium rationis expertium, ridiculum videcur,lapidem concretum in vefica hominissac frepe è renibus elapfúmac per penem redditum in nullo pretio haberi, cum tamen homo fitanimal longe nobilisfimum ac delicatisfimis cibis vefcatur. Sed Bontio,ut opinor,refponderi poffet: In herbis crudis,antidotis, quibus feræ illæ vefcuntur, plus effe virtutis ad ejusmodi lapides conftituendos? quam in delicatis hominum cibis.

2. Jom Succino Doer $\mathfrak{J e r n f t e i n}$.

Bermftein wiro Succinum, aud) Electrum, tho won Den 2rabern tor Carabe genant. $233 i r$ haben Derfele ben etliche Stricfe /, uno eines von 3. Yfunben / in Form eines Cylindresy Deffen Umbfang anberthall fuf. Sif vor 2. Sabren in ber 2seft:See am Noroftrande unferm bon Sider'fett/an einem: ungewoofntichen Sort gefunden novben. Won feiner generation Der moraus er entfethe / feunb unter: fchiedliche Nelununge/derer etliche $\mathrm{C} 2$ falpinus de Metallicis lib.2.cap. 28 . ergehlet. Athanafius Kircherus in arte Magnet. lib.3.cap.3. mie audh in Mundo fubterran: lib.8. c.3. bailt mit etlichen anbermbafúr/ Daß es ex bitumine fo bie Erobe auffwirffit/ entftefe/ unb gibt Darvon eine folche Befforeis bung: Succinum eft bitumen fubter raneum ex appropriatæ materiæ terreftris matrice fcaturiens, per fundum maris riparumq; aut fum:- 
nis appulfu mari concreditum, ibiq; ad littora appulfum, innata folis aerisq; ficcitate in lapideam fubftantiam excrefcit. Nemlid Der $\mathfrak{B e r m f t e i n}$ Faim aus Den Stjok ber Erben / gleich swie Das JubenpechNaffe und Petroleum, und wenn eśaus bem Mreer am

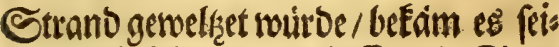
ne Sartigfeit bon Der \&uft uno Son nen Sithe. Dap aber in ettichen Stúcten (berer wir aud) baben) Mu: cfen/21meiren/Spinnen / Eiberen und Dergleichen gefunden werden / Eaim ber; wenn Der Sjernftein auß Dem 2Saffer auffoen Strano zu liegen Eém uno noch weidy/festen fich folche Shters lein brauff und bleiben betleben / monn. bann die See folche Stuifen mieber ergriffe / triebe uno íchluge Das 23 affer Den SBernftein theils in runbe theils in ander Formen/Dapalfo biefe Shietlein mitten binnein georucfet múrben / uno fo weit Kircherus. 2Aber wiber fors do seeynung Eonte man folgenoe ges Dancfen baben: Sft Der Sernftein eine 2irt bituminis ooer Jubenpech, und entffefet aus Der Erden/fo muiroen nicht allein in ber \$1t:See/fonbern auch nes ben Derfelben ober in Derfellen Biegend im aufgraben foldbe Etúcfe ober 2loen gefunden werben/ welches man gleichs molnichtuernommen. 230 lee man ifm troar bierinnen SEenfall geten/weil ots liche andere auch ber Meinung/ uno fas. gendấ an eflicten Sren in Deutfors. lano / Stalien und Franctreid etlide Stricten aús Der Eroen fennd gegraben norder/fo will Dod Das fchmer zu glátus ben fenn/ Dap Der SEernftein aufferfalb Dem 2 Saffer / wenn er weich an ben Strand aufseworffen muirbe/ erft von

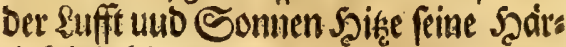
tigfeeit befám/uno wenner am Stranoe noct weich/bie Mhicken/Spinnen uns andere SGietlein in fich neljme/fo muilte folgen / baß aller 3ermftein nad) em. pfangener Şärte fith wieber in bie See begeber muife/Dann er mirb nicbt allein. am Strande fondern audb aus Der SRe mit शieģen fo hart auffeefif chet unto ges famlet. STtem es mufte auch nach ans geflebten Mucten nod) eine 23 eichige teit im Saccino. bleiben/Damit burch

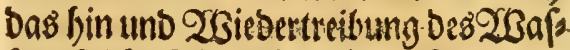
fers folche Thierlein/feiner soennung nach/mitten binnein getrieben roerben Eónten/und muffe hernads roieber an bie Eufft und Sonne / Dakes vollend ju. Stein muirbe. 2ther fdswertids wiro eine Seidste Mnusfe bie auffen anEtetets. Durb Daf bin uno mieber trcillen Deg 2Saffer: binein gettreben/vielmefre as bet abigetwafchen oder ver/fiummelt wer. ben. Alber wazumb laiffet at biefen nicht allein bas Principium Chymi. cum gelten? Cum Chymicis notum fit; Spiritum falis non folum alios. Spiritus fed \& oleofa quxvis conge. lare \& coagulare. Mnan wirb auch Das Sals Darinnen finden / menn mare Iiii

Den. 
Den Spiritum úbergiefet / To ferget fich Das Salman Den Saal $\&$ hoc eft â quo coagulatio facta eft. (ppridjt Olaus Worm)

Thb halte es aber in biefem Fall mit Dem Carfalpino und etticten anbern/

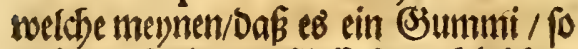

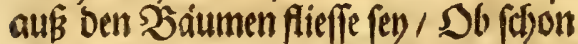
Kircherus faget/es wáre hetandt/ Daß in Эreuffen am ßaltifonen Mreer/ba Der Serntein fo háuffig gefamlet wird/ feine Sart ober Bummi tragende B̧äume gefunden werben. 21ber es if su miffen/Dapim Norbers Sheile Det See uno auff Den groffen Sinfein Bjottlano uno Deblano Sacume úlerflüfig / uns wajedset an eflichenSorten bie fruirmenbe See das bohe uffel alfo hinmeg/Dakes auch gankse 2báume mit binweg reiffet.

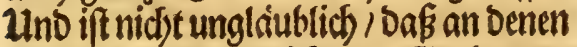
Srten / poúber etlicfe 60. Sirad yom Aquatore, ba bes Sommers bie Solk ne eftliche Mzonat uiter Dem Horizont bleibet / uno gar nicht untergethet / ihte continuirliche Shige Die Sichten / Dans nen und 23 acholder Sarume / po Das felboft gro $\mathfrak{\beta}^{2}$ untereinander in fehr groffer Menge wachjen / Dap fie bie refinam uno Summi báuffig bon fich fieffien lafs Fen/Die in groffe Rlumpen treuffeln $/ \mathrm{ba}$ Dann Mucfen uns anber: gefdomeiffe Dartswifisen fonumen Eónnen / wieich Dann forches in Carelien ben ber $\mathfrak{V}_{e}$ ftung??oleburg úber 60.grad Elev.poli auffeiner in Der \&abogaifden See liegenden Sinfel (Der in Der \$nufondir

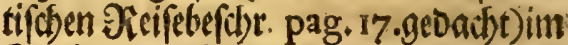
Stunio / Da Die Sonne zu Mitternadbt Eaum an Den Horizont ftriche/felloft ges fehen / Dẩ aus ettichen Bäumen Das

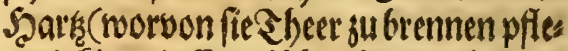
gen) Elar als Evprifher Serpentim/von etfichen trúbe uno Dunctel heraus flof. QSenn nun folches ourd) Sturm oder andere Zufálle in bie See Eommt / wiro: es burd Das Sals: 2Baffer gethártet! und alfo nol etliche 100. Jahe in Der See gewailset uno gettieben / bík es zu Eano Eommt / uno wito nicbt allein in Breuffen / fondern auds ! mie Olaus. Worm istreibet / fáuffig an ber 233 es ften Seiten Sutlanbes gefunden.

Eines if an Dem Sernftein luftig ans. surehen/Das et eime Magnetiche Sraffit infich Gabe/unt gleid) wie Der \$lagnet DageEifen/2Btumen/alfo aud) Dez2Bern: ftein allerhano leidfte Sachen / alö Ses: Dern/ Eiferne unbanber Mretallen Feils fpune und Sanb an fíd ziefer / ja bas noch mefo zu verwunbern : Er ziefyet nirft allein bie leichte Sachen / fondern audb Eifen/Golb/Silber/ Supffer / obo fobon fobwer ift / nach fich / wenn man nemblid baffer lbe in Der Mitte entweber an einen Faben bainget Doer auff eine Spiser / gleid wie eine Mragnets?atel ftellet / baß́三 es fith auff benden Seiten frey) berwegen Ean/ids habe es mit etlicher Eeute grofier 2 erwunberung mit einem

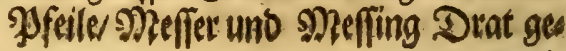
senget. 
zeiget. Der ßènftein muß̧ aber juvor eine 28 sarme empfangen. 2 Aber es gilt Dod) nicht gleid) / ourd) was SMittel Der Bernftein erwármet wirb/wenn es von Der Sonnen/ beym Feur / in Der Şand vom marmen waffer gefchiehet / wiro er bas nach fich ilisem alio nictst vertichs ten / als swenn ex auff einem tructenen 2sulden Tudje gerieben und erhisiget wirb. Es mußs aber bas Electrum nid)t rauds/fonbern glat poflietet feyn. Da a

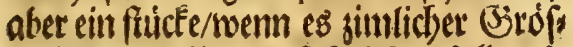
fe if / b bne reiben an fich siefsen foll / wie Cabeus fchreibet lib.2.cap.18.Philor, Magn, babe ich an unferm Drenpfuntois gem nidjt mercfen Eónnen / es múfte Denn Lrf Fache feys/meil es nidjt gar flar! fonberngefluno trúbe / uno nicht polis ret.

Tab babe aud befunden/Dak Das Asphaltum ober Subenpect) / Stem/ Das flare Gummi Animæ, fonft Aimæ genant/menn fie am sucbe geries. ben werben/fo mol als Das Succinum ober Sbernfeein allerfiano nach fich zies be / fo mol auffs und unterwert als zu Den Seiten/menn bas objectum in $x$ quilibrio fanget/mietwol ifre stafft im siefen etwas cotwaider ifi. Shb babe aud) Darbey befunben/Dás Daš an fich ziefen in allen breyen obgebactsen Sturfennictst to wol in gar heiffer ober nuch gar feucter/alo Ealter uno Elarer suffit geforjiefiet.
Die Urfachenaber / Dás Die Electra allerfyano Sachen nach fid ziefen/wers Den von Den Autoren unterfóieblids gegeben. Die vornebmiten Mesnum: gen aber/fo bey Den Phyficis in confideration Eommen fónnen / Ferno Des Gilberti, Cabei uns Kircheri. Gilbertus meynet: quod ex electro tanquam ex corpore ex humore concreto exeat f́piritus five effluvium (effluvia verò non funt flatus, fed humores fumme attenuati,aëre ambiente multò tenuiores, ficut aër dicitur commune effluvium telluris) quo fibi adjungit \& attrahit $\alpha$ mnia corpora. Diefe Nennung wito vom Cabeo uno Kirchero refutiret/ Derfelben Rationes mit einjufúfren! múrbe zu weitldiuftig fallen. Cabxi Neeynung aber ift aus folgenden zuver: nefmen. (lib. 2. cap. 2I.) Dico igitur ex Electro, feu ex quolibet corpore attrahente electricè (calore per fricationem in fuperficie excitato) quando fic attrahit, effluere effluvium tenuiffimum, quod aërem attenuat, \& dejicit, imo \& incitatifime impellit, fed tenuiter; tum vero attenuatus \& impulfus aër revertitur ad corpus electricum guafi in gyrum (uti ejusmodi motus apparet in atomis fole per feneftram ingrediente) fecumque una rapit paleas, \& quacunque obvia corpufcula. Sed corpus: 
corpus debet effe terfum \& nitidum nulla ratione obnubilatum autaqua aut halitu humido. Sicut enim per moderatum illum calorem aperiuntur pori ut exire poffit illud effluvium, ita per humidum halitum obduratur ftatim \& impeditur tranfpiratio. Hinc eft, ut opus fit experimentum attractionis fieri, cælo fereno, puro, non humido aut nebulofo. Et fricatio non tam requiritur ad excitandum calorem, quam ad detergendum; \& ideo quodlibet electri fruftum competentis magnizudinis unica folum terfione efficaciori fufficienter præparatur ad tra. hendum ut frep fum expertus; \& fi bene terfum electrum cuftodiatur ne aut pulvere aut humido halitu inficiatur, poft duodecim aut quindecim horas trahet adhuc efficaciter. Hinc eft, quod non trahet electrum, calore folis, aut aquâ calida calefactum, fed calore per fricationem excitato, non quod ille calor fit diverfus, fed quia fic exquifitiffme torgitur ab humida nubecula \& fordibus aqueis.
Athan. Kircheri gneynung ift Des Cabeinic)t fefor ungleid)/weñ er foget: lib.3. parte 3. cap. 3. Mundi five catence magne: Dico igitur; $\sqrt{2}$ corpus aliquod ex pingui bumore concretum, adeo tenui atg. fubtili effluvio confttterit, id ad quemvir motum levem feu terfionem fuperficialem concisatum calefactumg, è veftigio tenuisfimum illud fuum effluvium emittere; quod cum rarum fit is admodum tenue, bac fue varìtatem aêrem quog circumfitum, prefertion frigidiufculum attenuatum disjicere, disjectum comprimere, Sed pro tenuirate effluvii, tenuiter; aër vero attenuatus compre $3 u s$ disjectusg, refätentibus aliis continustis aêris frigidioribus partibus ad corpus electricum revertitur, quem aëris retrocedentis motum paleas quog ${ }_{3}$ o obvia quavis leviora corpufcula velutitracta comitari neceffe eft.

2 Baz bietwon Cardani Megnung uno wie fellige bom Scaliger wibers fprodjen wiro / uno was Deffen eigene s)eynung / ift Exerc, 104, cap.4.34 lefen.

Uno foviel auf bifmahlson uns fer Bjottorfficton Sin Rammer.

\section{$\mathfrak{E} \mathfrak{R} \mathfrak{D} \mathfrak{E}$}








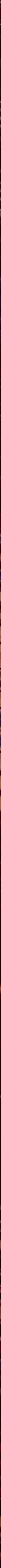




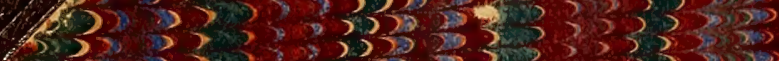

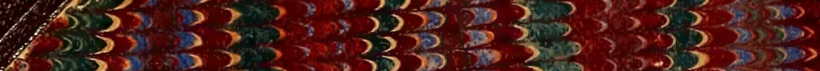

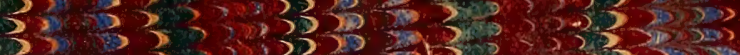

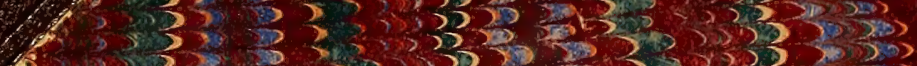

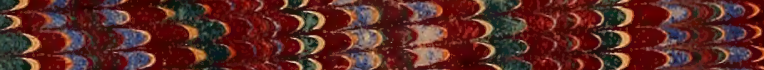

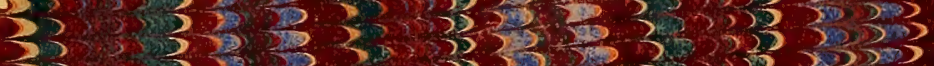

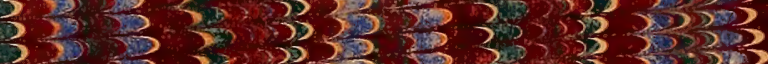

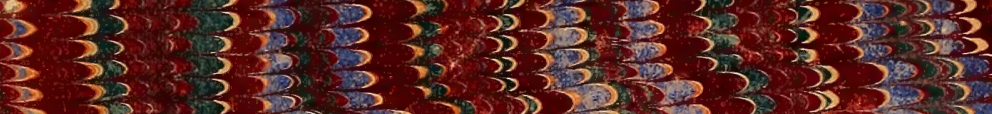

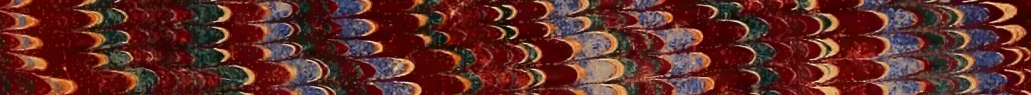

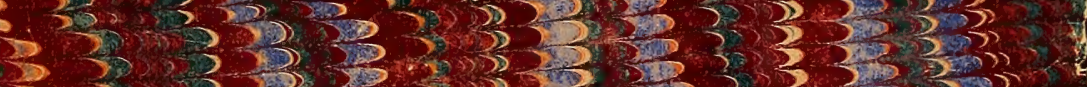

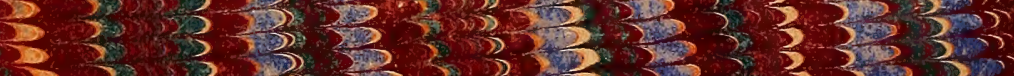

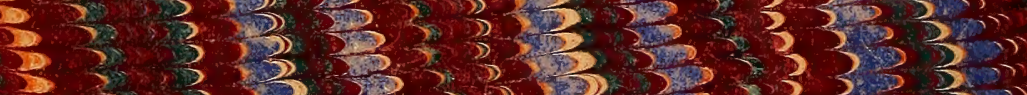

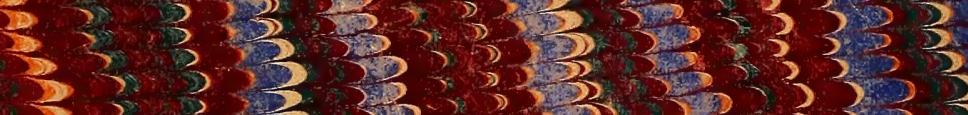

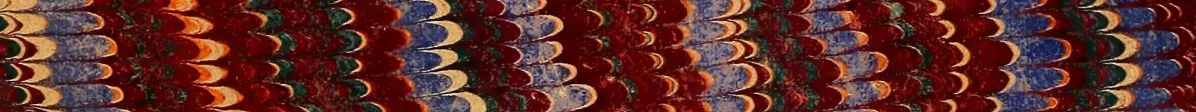

329

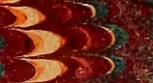

3203

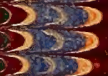

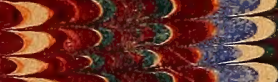
$30,2,3$

3 $33^{2}$ $20 x$ 3302 3

235 ?

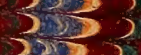

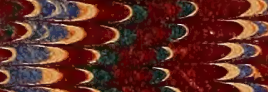

3,3

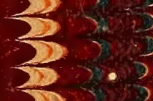

$\int_{3}$

3

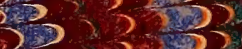

$\sum$

$3 x^{3}$

(2)

3,3

3

25

$3_{3}^{3} \sum_{3}^{3} \quad \frac{3}{3}$

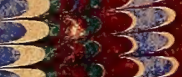

(5) 53

3035

) 2.5

$2)$ 에

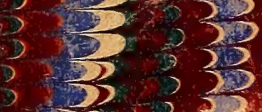

$\frac{1}{3}+\frac{3}{3}, 3$

$30,9,201$

5) 39

,

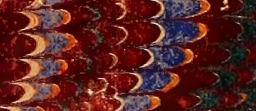

$\left.3)^{3}\right)^{3}=$

.
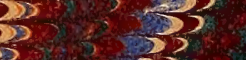

, 3.
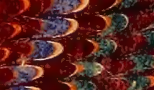

(

.

33

$3,3,3.3$

$3,3,5,3,3$

$\rightarrow \frac{3}{3}, 5,5$

33

23

$b_{3}$

(5) $3,5, \frac{3}{3}$

2

32

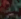

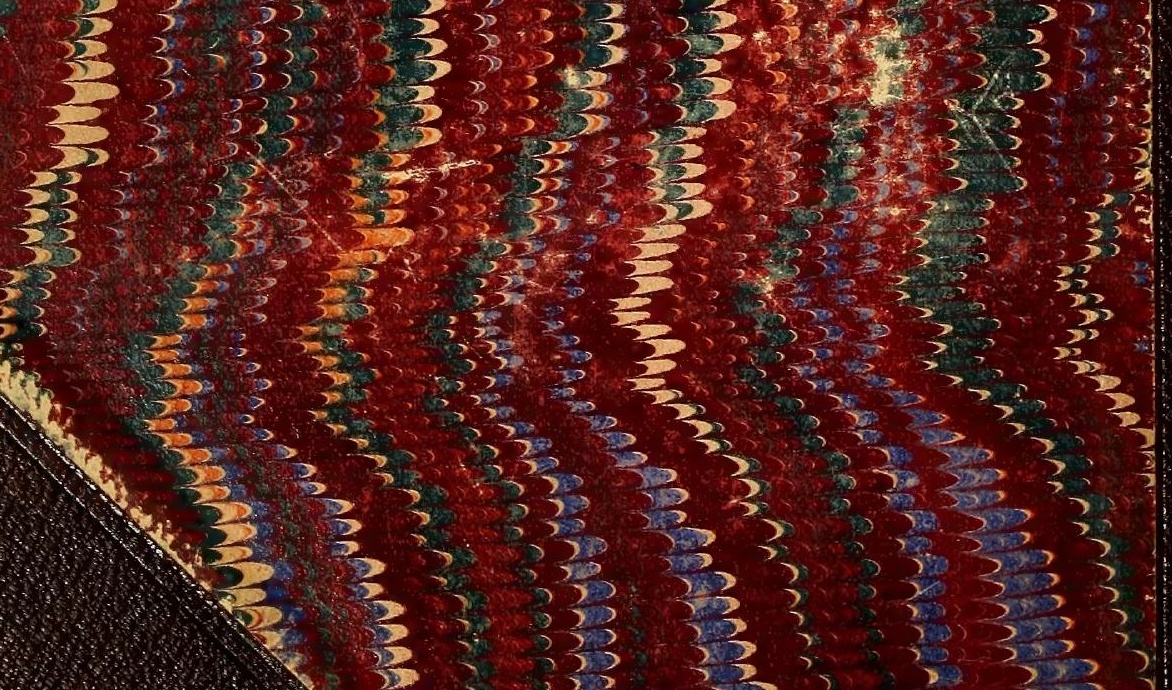

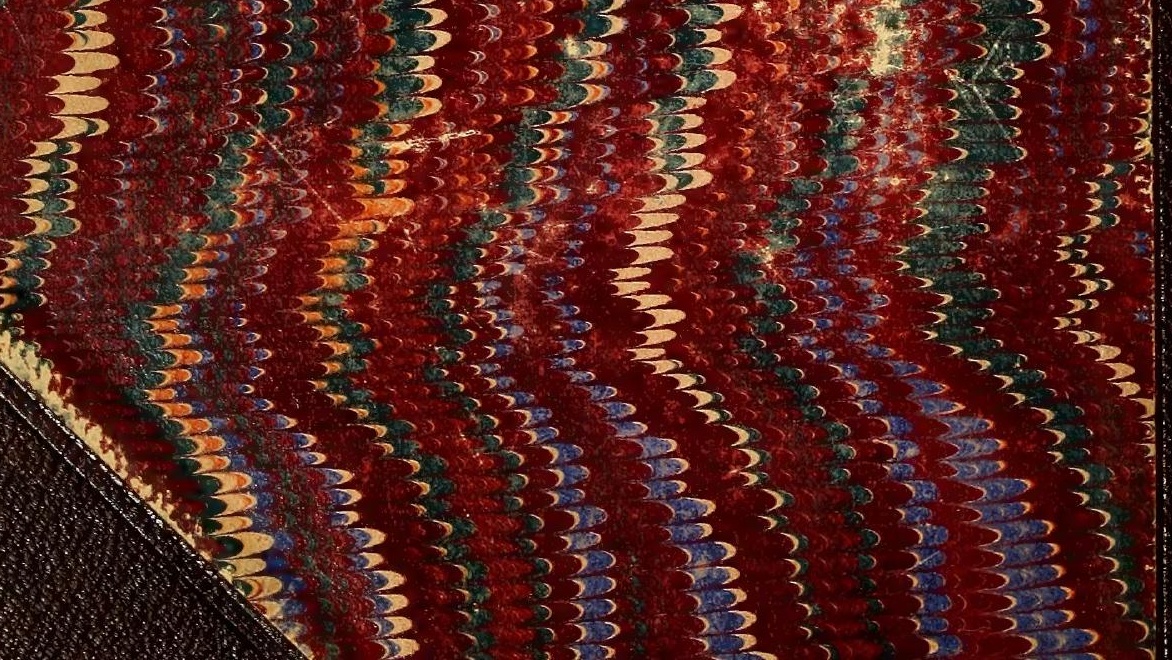

$3.93 \times 9$, 5.

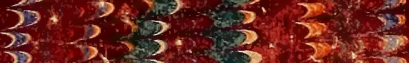

3.32

$3 \times 3 \div 2$

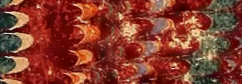

$\frac{3}{3}: \frac{53}{2}, \frac{3}{3}$

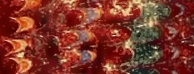

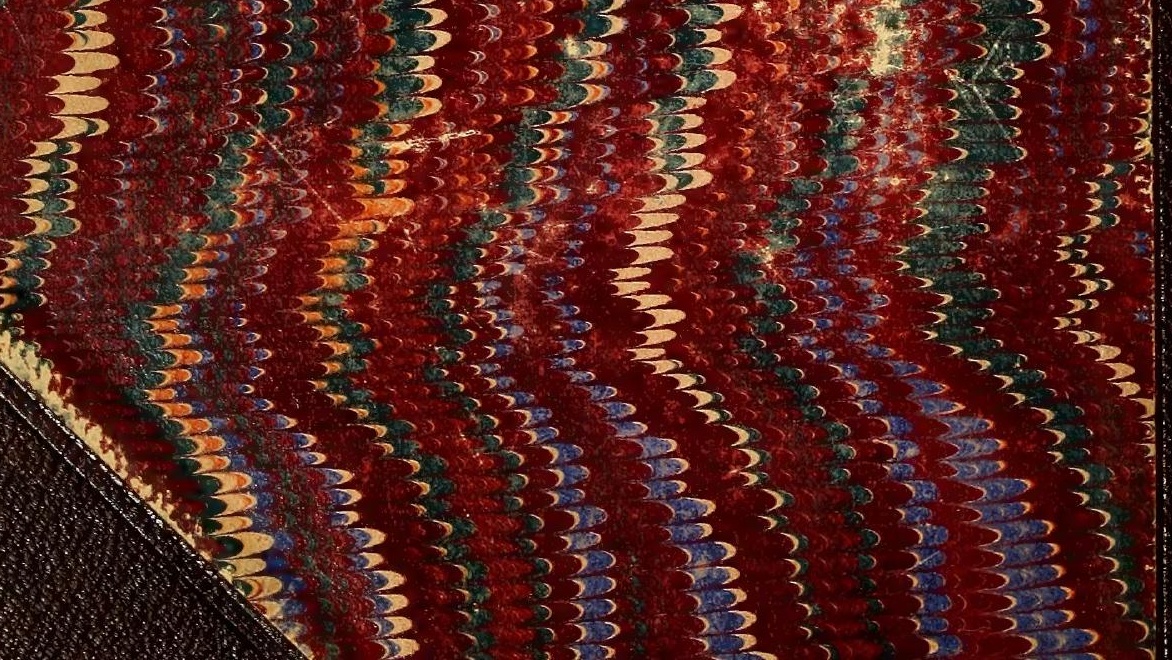

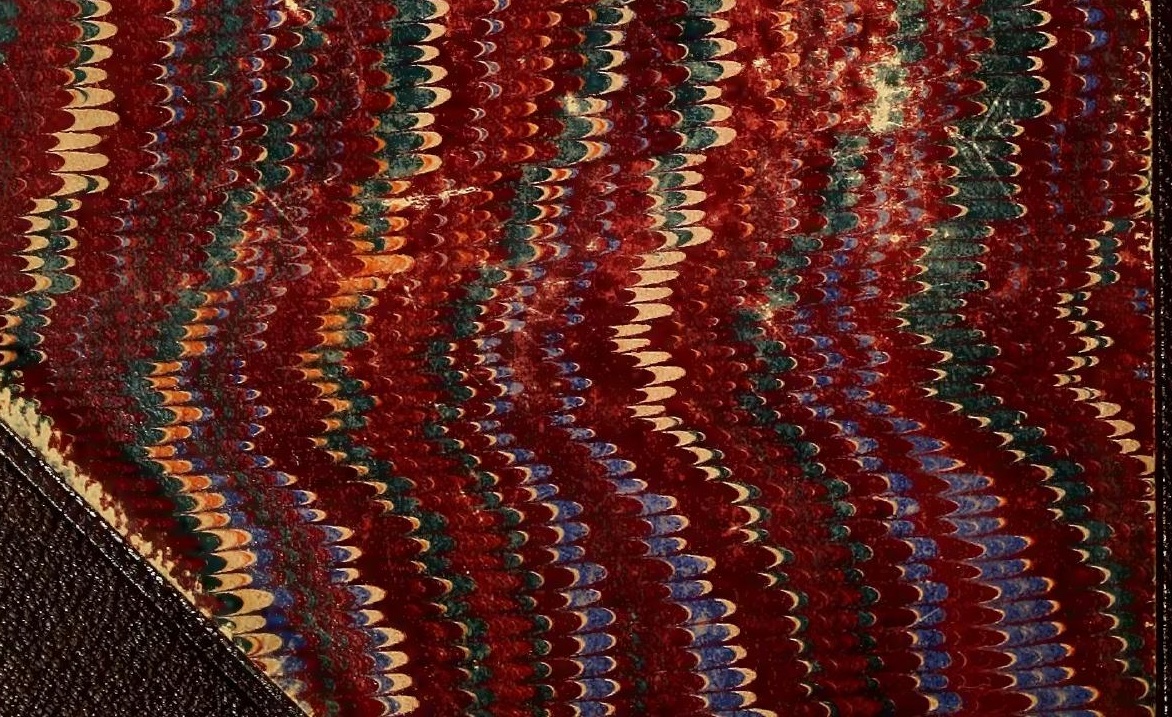

3,3

33

33

$33_{3}$

$3>3$

3.34

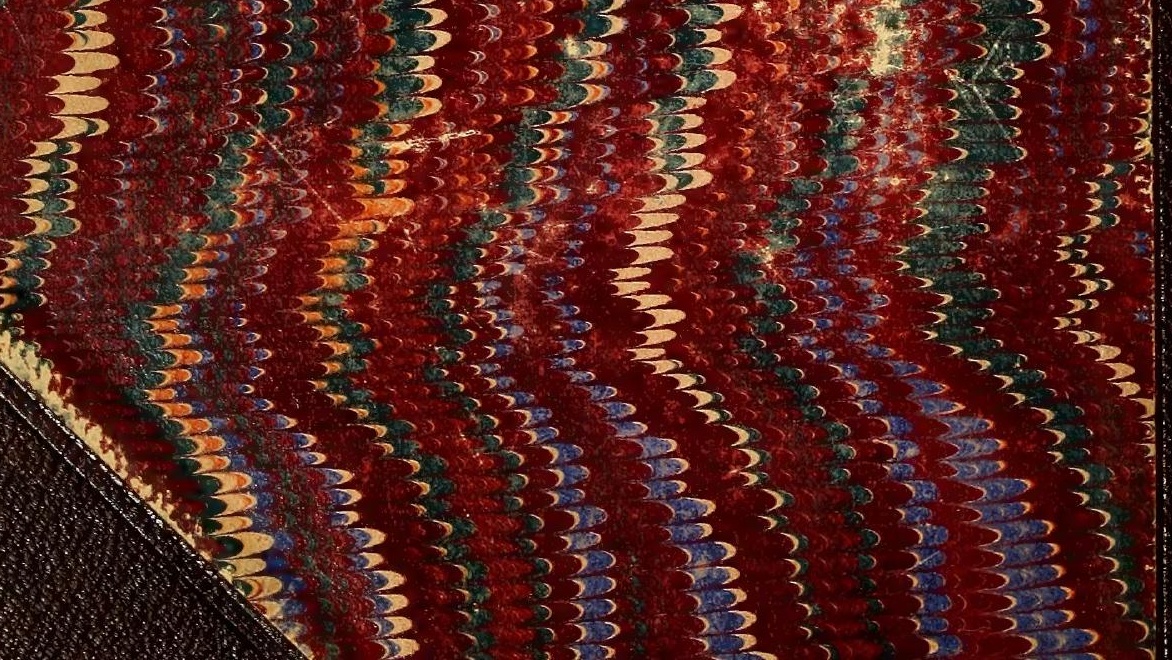

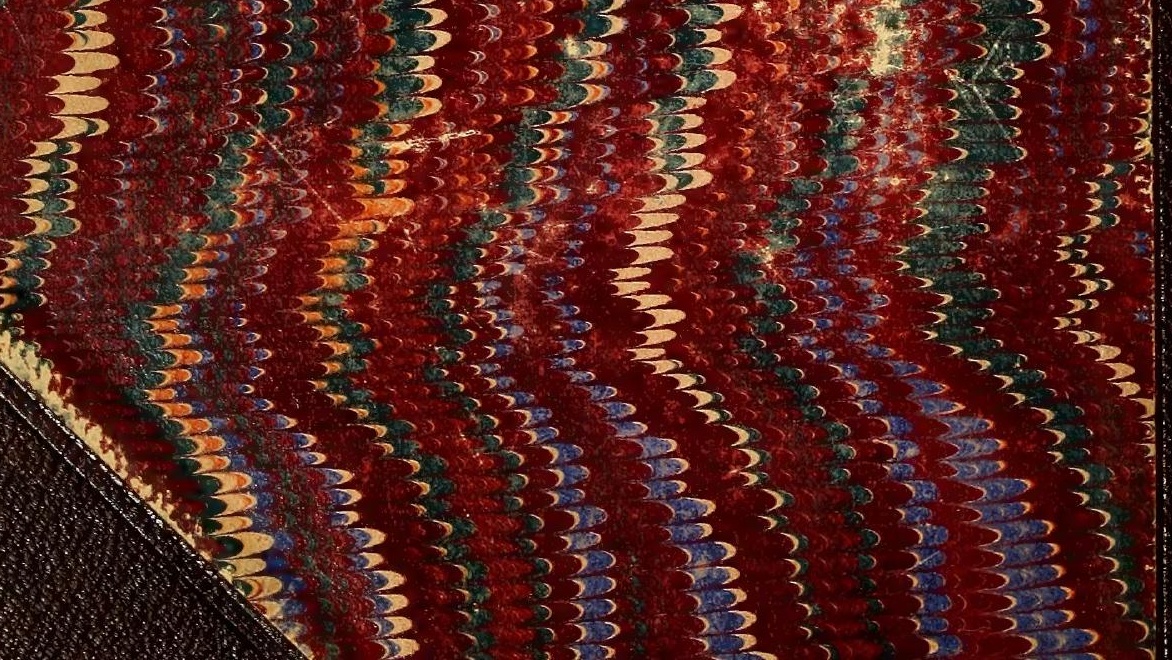

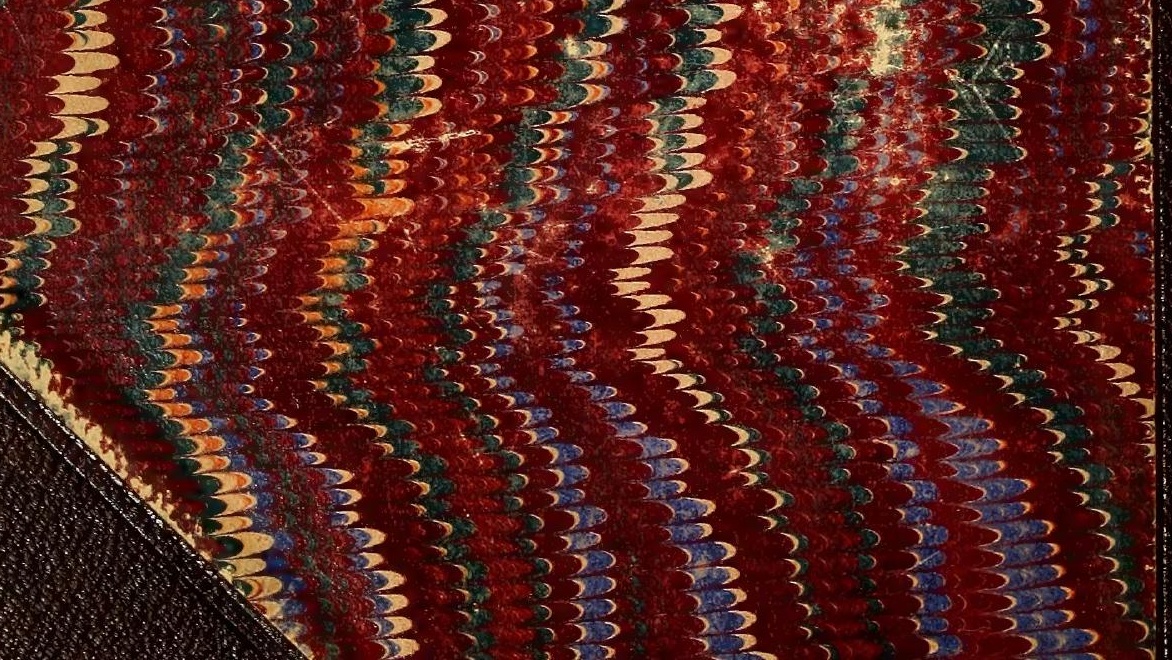

